Cochrane Database of Systematic Reviews

\title{
Cognitive behavioural therapy for tinnitus (Review)
}

Fuller T, Cima R, Langguth B, Mazurek B, Vlaeyen JWS, Hoare DJ

Fuller T, Cima R, Langguth B, Mazurek B, Vlaeyen JWS, Hoare DJ.

Cognitive behavioural therapy for tinnitus.

Cochrane Database of Systematic Reviews 2020, Issue 1. Art. No.: CD012614.

DOI: 10.1002/14651858.CD012614.pub2.

www.cochranelibrary.com 
TABLE OF CONTENTS

PLAIN LANGUAGE SUMMARY

SUMMARY OF FINDINGS

BACKGROUND

OBJECTIVES

METHODS

Figure 1.

RESULTS

Figure 2.

Figure 3.

Figure 4.

Figure 5.

Figure 6.

DISCUSSION

AUTHORS' CONCLUSIONS

ACKNOWLEDGEMENTS

REFERENCES

CHARACTERISTICS OF STUDIES

DATA AND ANALYSES

Analysis 1.1. Comparison 1 CBT versus no intervention/waiting list control, Outcome $1 \mathrm{Impact}$ of tinnitus on quality of life at end of treatment.

Analysis 1.2. Comparison 1 CBT versus no intervention/waiting list control, Outcome 2 Serious adverse effects at end of treatment.

Analysis 1.3. Comparison 1 CBT versus no intervention/waiting list control, Outcome 3 Depression at end of treatment. ........ Analysis 1.4. Comparison 1 CBT versus no intervention/waiting list control, Outcome 4 Anxiety at end of treatment. .............. Analysis 1.5. Comparison 1 CBT versus no intervention/waiting list control, Outcome 5 Health-related quality of life at end of treatment.

Analysis 1.6. Comparison 1 CBT versus no intervention/waiting list control, Outcome 6 Negatively biased interpretations of tinnitus at end of treatment.

Analysis 1.7. Comparison 1 CBT versus no intervention/waiting list control, Outcome 7 Subgroup analysis (random-effects model): type of therapy - impact of tinnitus on quality of life at end of treatment.

Analysis 1.8. Comparison 1 CBT versus no intervention/waiting list control, Outcome 8 Subgroup analysis (fixed-effect model): type of therapy - impact of tinnitus on quality of life at end of treatment.

Analysis 1.9. Comparison 1 CBT versus no intervention/waiting list control, Outcome 9 Subgroup analysis (random-effects model): mode of delivery - impact of tinnitus on quality of life at end of treatment.

Analysis 1.10. Comparison 1 CBT versus no intervention/waiting list control, Outcome 10 Subgroup analysis (fixed-effect model): mode of delivery - impact of tinnitus on quality of life at end of treatment.

Analysis 1.11. Comparison 1 CBT versus no intervention/waiting list control, Outcome 11 Subgroup analysis (random-effects model): unit of delivery - impact of tinnitus on quality of life at end of treatment.

Analysis 1.12. Comparison 1 CBT versus no intervention/waiting list control, Outcome 12 Subgroup analysis (fixed-effect model): unit of delivery - impact of tinnitus on quality of life at end of treatment.

Analysis 1.13. Comparison 1 CBT versus no intervention/waiting list control, Outcome 13 Subgroup analysis (random-effects model): who delivers CBT - impact of tinnitus on quality of life at end of treatment.

Analysis 1.14. Comparison 1 CBT versus no intervention/waiting list control, Outcome 14 Subgroup analysis (fixed-effect model): who delivers CBT - impact of tinnitus on quality of life at end of treatment.

Analysis 1.15. Comparison 1 CBT versus no intervention/waiting list control, Outcome 15 Sensitivity analysis without Malinvaud (high risk of bias) impact of tinnitus on quality of life at end of treatment.

Analysis 1.16. Comparison 1 CBT versus no intervention/waiting list control, Outcome 16 Sensitivity analysis with optimistic assumption for Malinvaud - impact of tinnitus on quality of life at end of treatment.

Analysis 2.1. Comparison 2 CBT versus audiological care (tinnitus education and rehabilitation for hearing loss), Outcome 1 Impact of tinnitus on quality of life at end of treatment.

Analysis 2.2. Comparison 2 CBT versus audiological care (tinnitus education and rehabilitation for hearing loss), Outcome 2 Serious adverse effects at end of treatment. 
Analysis 2.3. Comparison 2 CBT versus audiological care (tinnitus education and rehabilitation for hearing loss), Outcome 3 Depression at end of treatment.

Analysis 2.4. Comparison 2 CBT versus audiological care (tinnitus education and rehabilitation for hearing loss), Outcome 4 Anxiety at end of treatment.

Analysis 2.5. Comparison 2 CBT versus audiological care (tinnitus education and rehabilitation for hearing loss), Outcome 5 Health-related quality of life.

Analysis 2.6. Comparison 2 CBT versus audiological care (tinnitus education and rehabilitation for hearing loss), Outcome 6 Negatively biased interpretations of tinnitus.

Analysis 2.7. Comparison 2 CBT versus audiological care (tinnitus education and rehabilitation for hearing loss), Outcome 7 Sensitivity analysis (fixed-effect model): impact of tinnitus on quality of life.

Analysis 3.1. Comparison 3 CBT versus TRT (directive counselling and bilateral masking), Outcome 1 Impact of tinnitus on quality of life at end of treatment.

Analysis 3.2. Comparison 3 CBT versus TRT (directive counselling and bilateral masking), Outcome 2 Impact of tinnitus on quality of life at 6 months follow-up.

Analysis 3.3. Comparison 3 CBT versus TRT (directive counselling and bilateral masking), Outcome 3 Serious adverse effects at end of treatment.

Analysis 3.4. Comparison 3 CBT versus TRT (directive counselling and bilateral masking), Outcome 4 Negatively biased interpretations of tinnitus at end of treatment.

Analysis 3.5. Comparison 3 CBT versus TRT (directive counselling and bilateral masking), Outcome 5 Negatively biased interpretations of tinnitus at 6 months follow-up.

Analysis 4.1. Comparison 4 CBT versus other active control, Outcome $1 \mathrm{Impact}$ of tinnitus on quality of life at end of treatment. Analysis 4.2. Comparison 4 CBT versus other active control, Outcome 2 Impact of tinnitus on quality of life at 6 months followup.

Analysis 4.3. Comparison 4 CBT versus other active control, Outcome 3 Impact of tinnitus on quality of life at 12 months followup.

Analysis 4.4. Comparison 4 CBT versus other active control, Outcome 4 Serious adverse effects at the end of treatment. ........

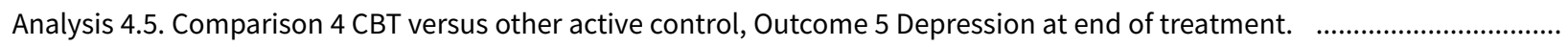
Analysis 4.6. Comparison 4 CBT versus other active control, Outcome 6 Depression at 6 months follow-up. ............................ Analysis 4.7. Comparison 4 CBT versus other active control, Outcome 7 Depression at 12 months.

Analysis 4.8. Comparison 4 CBT versus other active control, Outcome 8 Anxiety at end of treatment.

Analysis 4.9. Comparison 4 CBT versus other active control, Outcome 9 Anxiety at 6 months follow-up.

Analysis 4.10. Comparison 4 CBT versus other active control, Outcome 10 Health-related quality of life at end of treatment. ...

Analysis 4.11. Comparison 4 CBT versus other active control, Outcome 11 Negatively biased interpretations of tinnitus at end of treatment.

Analysis 4.12. Comparison 4 CBT versus other active control, Outcome 12 Negatively biased interpretations of tinnitus at 6 months follow-up.

Analysis 4.13. Comparison 4 CBT versus other active control, Outcome 13 Negatively biased interpretations of tinnitus at 12 months follow-up.

Analysis 4.14. Comparison 4 CBT versus other active control, Outcome 14 Subgroup analysis (random-effects model): type of therapy - impact of tinnitus on quality of life.

Analysis 4.15. Comparison 4 CBT versus other active control, Outcome 15 Subgroup analysis (fixed-effect model): type of therapy - impact of tinnitus on quality of life.

Analysis 4.16. Comparison 4 CBT versus other active control, Outcome 16 Subgroup analysis: mode of delivery - impact of tinnitus on quality of life.

Analysis 4.17. Comparison 4 CBT versus other active control, Outcome 17 Subgroup analysis: unit of delivery -impact of tinnitus on quality of life.

Analysis 4.18. Comparison 4 CBT versus other active control, Outcome 18 Subgroup analysis: who delivers intervention - impact of tinnitus on quality of life.

Analysis 4.19. Comparison 4 CBT versus other active control, Outcome 19 Subgroup analysis: type of control - impact of tinnitus on quality of life.

Analysis 4.20. Comparison 4 CBT versus other active control, Outcome 20 Sensitivity analysis (fixed-effect model): impact of tinnitus on quality of life at end of treatment.

Analysis 4.21. Comparison 4 CBT versus other active control, Outcome 21 Sensitivity analysis: without studies at high risk of bias for incomplete outcome data - impact of tinnitus on quality of life.

Analysis 4.22. Comparison 4 CBT versus other active control, Outcome 22 Sensitivity analysis: without high risk of bias of missing outcome data, by subgroups (random-effects model): type of therapy - impact of tinnitus on quality of life. 
Analysis 4.23. Comparison 4 CBT versus other active control, Outcome 23 Sensitivity analysis: optimistic assumption for Malinvaud - impact of tinnitus on quality of life.

Analysis 4.24. Comparison 4 CBT versus other active control, Outcome 24 Sensitivity analysis: optimistic assumption for Malinvaud - depression.

Analysis 4.25. Comparison 4 CBT versus other active control, Outcome 25 Sensitivity analysis: optimistic assumption for Malinvaud - anxiety.

APPENDICES

CONTRIBUTIONS OF AUTHORS 
[Intervention Review]

\section{Cognitive behavioural therapy for tinnitus}

Thomas Fuller1,2,3a, Rilana Cima1,2, Berthold Langguth4, Birgit Mazurek ${ }^{5}$ Johan WS Vlaeyen 6 , Derek J Hoare ${ }^{7}$

1Department of Clinical Psychological Science, Maastricht University, Maastricht, Netherlands. 2Adelante, Centre for Expertise in Rehabilitation \& Audiology, Hoensbroek, Netherlands. ${ }^{3}$ Spine and Biologics, Medtronic, Maastricht, Netherlands. ${ }^{4}$ Department of Psychiatry and Psychotherapy, University of Regensburg, Regensburg, Germany. ${ }^{5}$ Charité - Universitätsmedizin Berlin, Berlin, Germany. ${ }^{6}$ Research Group Health Psychology, KU Leuven University, Leuven, Belgium. ${ }^{7}$ NIHR Nottingham Biomedical Research Centre, Division of Clinical Neuroscience, School of Medicine, University of Nottingham, Nottingham, UK

${ }^{a}$ As of 1 October 2019, Thomas Fuller holds a position at Medtronic as a Senior Clinical Evidence Specialist in addition to his existing affiliations with Maastricht University and Adelante.

Contact: Thomas Fuller, Department of Clinical Psychological Science, Maastricht University, Universiteitssingel 40, Maastricht, 6200 MD, Netherlands. thomasefuller@yahoo.co.uk, thomas.fuller@maastrichtuniversity.nl.

Editorial group: Cochrane ENT Group.

Publication status and date: New, published in Issue 1, 2020.

Citation: Fuller T, Cima R, Langguth B, Mazurek B, Vlaeyen JWS, Hoare DJ. Cognitive behavioural therapy for tinnitus. Cochrane Database of Systematic Reviews 2020, Issue 1. Art. No.: CD012614. DOI: 10.1002/14651858.CD012614.pub2.

Copyright @ 2020 The Cochrane Collaboration. Published by John Wiley \& Sons, Ltd.

\section{A B S T R A C T}

\section{Background}

Tinnitus affects up to $21 \%$ of the adult population with an estimated $1 \%$ to $3 \%$ experiencing severe problems. Cognitive behavioural therapy (CBT) is a collection of psychological treatments based on the cognitive and behavioural traditions in psychology and often used to treat people suffering from tinnitus.

\section{Objectives}

To assess the effects and safety of CBT for tinnitus in adults.

\section{Search methods}

The Cochrane ENT Information Specialist searched the ENT Trials Register; CENTRAL (2019, Issue 11); Ovid MEDLINE; Ovid Embase; CINAHL; Web of Science; ClinicalTrials.gov; ICTRP and additional sources for published and unpublished trials. The date of the search was 25 November 2019.

\section{Selection criteria}

Randomised controlled trials (RCTs) of CBT versus no intervention, audiological care, tinnitus retraining therapy or any other active treatment in adult participants with tinnitus.

\section{Data collection and analysis}

We used the standard methodological procedures expected by Cochrane. Our primary outcomes were the impact of tinnitus on diseasespecific quality of life and serious adverse effects. Our secondary outcomes were: depression, anxiety, general health-related quality of life, negatively biased interpretations of tinnitus and other adverse effects. We used GRADE to assess the certainty of evidence for each outcome. 


\section{Main results}

We included 28 studies (mostly from Europe) with a total of 2733 participants. All participants had had tinnitus for at least three months and their average age ranged from 43 to 70 years. The duration of the CBT ranged from 3 to 22 weeks and it was mostly conducted in hospitals or online.

There were four comparisons and we were interested in outcomes at end of treatment, and 6 and 12 months follow-up. The results below only refer to outcomes at end of treatment due to an absence of evidence at the other follow-up time points.

\section{CBT versus no intervention/wait list control}

Fourteen studies compared CBT with no intervention/wait list control. For the primary outcome, CBT may reduce the impact of tinnitus on quality of life at treatment end (standardised mean difference (SMD) $-0.56,95 \%$ confidence interval (Cl) -0.83 to $-0.30 ; 10$ studies; 537 participants; low certainty). Re-expressed as a score on the Tinnitus Handicap Inventory (THI; range 0 to 100) this is equivalent to a score 10.91 points lower in the CBT group, with an estimated minimal clinically important difference (MCID) for this scale being 7 points. Seven studies, rated as moderate certainty, either reported or informed us via personal communication about serious adverse effects. CBT probably results in little or no difference in adverse effects: six studies reported none and in one study one participant in the CBT condition worsened (risk ratio (RR) $3.00,95 \% \mathrm{Cl} 0.13$ to 69.87 ). For the secondary outcomes, CBT may result in a slight reduction in depression (SMD $-0.34,95 \% \mathrm{Cl}-0.60$ to -0.08 ; 8 studies; 502 participants; low certainty). However, we are uncertain whether CBT reduces anxiety, improves health-related quality of life or reduces negatively biased interpretations of tinnitus (all very low certainty). From seven studies, no other adverse effects were reported (moderate certainty).

\section{CBT versus audiological care}

Three studies compared CBT with audiological care. CBT probably reduces the impact of tinnitus on quality of life when compared with audiological care as measured by the THI (range 0 to 100; mean difference (MD) $-5.65,95 \% \mathrm{Cl}-9.79$ to $-1.50 ; 3$ studies; 444 participants) (moderate certainty; MCID = 7 points). No serious adverse effects occurred in the two included studies reporting these, thus risk ratios were not calculated (moderate certainty). The evidence suggests that CBT may slightly reduce depression but may result in little or no difference in anxiety or health-related quality of life (all low certainty) when compared with audiological care. CBT may reduce negatively biased interpretations of tinnitus when compared with audiological care (low certainty). No other adverse effects were reported for either group (moderate certainty).

\section{CBT versus tinnitus retraining therapy (TRT)}

One study compared CBT with TRT (including bilateral sound generators as per TRT protocol). CBT may reduce the impact of tinnitus on quality of life as measured by the THI when compared with TRT (range 0 to 100 ) (MD $-15.79,95 \% \mathrm{Cl}-27.91$ to $-3.67 ; 1$ study; 42 participants; low certainty). For serious adverse effects three participants deteriorated during the study: one in the CBT ( $n=22)$ and two in the TRT group $(n=20)$ (RR $0.45,95 \% \mathrm{Cl} 0.04$ to 4.64; low certainty). We are uncertain whether CBT reduces depression and anxiety or improves healthrelated quality of life (low certainty). CBT may reduce negatively biased interpretations of tinnitus. No data were available for other adverse effects.

\section{CBT versus other active control}

Sixteen studies compared CBT with another active control (e.g. relaxation, information, Internet-based discussion forums). CBT may reduce the impact of tinnitus on quality of life when compared with other active treatments (SMD $-0.30,95 \% \mathrm{Cl}-0.55$ to $-0.05 ; 12$ studies; 966 participants; low certainty). Re-expressed as a THI score this is equivalent to 5.84 points lower in the CBT group than the other active control group ( $M C I D=7$ points). One study reported that three participants deteriorated: one in the CBT and two in the information only group (RR 1.70, 95\% Cl 0.16 to 18.36; low certainty). CBT may reduce depression and anxiety (both low certainty). We are uncertain whether CBT improves health-related quality of life compared with other control. CBT probably reduces negatively biased interpretations of tinnitus compared with other treatments. No data were available for other adverse effects.

\section{Authors' conclusions}

CBT may be effective in reducing the negative impact that tinnitus can have on quality of life. There is, however, an absence of evidence at 6 or 12 months follow-up. There is also some evidence that adverse effects may be rare in adults with tinnitus receiving CBT, but this could be further investigated. CBT for tinnitus may have small additional benefit in reducing symptoms of depression although uncertainty remains due to concerns about the quality of the evidence. Overall, there is limited evidence for CBT for tinnitus improving anxiety, healthrelated quality of life or negatively biased interpretations of tinnitus.

\section{PLAIN LANGUAGE SUMMARY}

\section{Cognitive behavioural therapy for adults with tinnitus}

\section{What is the aim of this review?}


The aim of this Cochrane Review was to find out if cognitive behavioural therapy (CBT) is effective for tinnitus. Cochrane researchers collected and analysed all relevant studies to answer this question.

\section{Key messages}

There is some low- to moderate-certainty evidence that CBT may reduce the negative impact that tinnitus can have on quality of life at the end of treatment, with few or no adverse effects (although further research on this is needed).

\section{What was studied in the review?}

Tinnitus is the perception of sound in the ear or head without any outside source. It is often described as a ringing, hissing, buzzing or whooshing sound. Tinnitus is mostly managed with education and/or counselling, relaxation therapy, tinnitus retraining therapy and earlevel sound generators or hearing aids. CBT is a form of talking therapy that aims to change the patient's emotional and/or behavioural response to their tinnitus. This review looked at studies of CBT for adults who had had tinnitus for at least three months. Participants in the control groups either received no intervention, audiological (hearing) care, tinnitus retraining therapy or another type of treatment. The review authors studied the effect of CBT on tinnitus-related quality of life, adverse effects, depression, anxiety, general quality of life and negatively biased interpretations of tinnitus.

\section{What are the main results of the review?}

We found 28 relevant studies, mostly from Europe, with a total of 2733 participants. The participants receiving CBT had treatment for between three and 22 weeks (mostly in clinics or online).

When CBT was compared to no intervention there was low-certainty evidence that CBT may reduce the negative impact of tinnitus on quality of life at the end of treatment. It is not known whether this effect persists in the longer term (six or 12 months). There were few or no adverse effects (only one adverse effect was reported in one participant among seven studies). CBT may also slightly reduce depression (low-certainty evidence) and may reduce anxiety, although this finding is very uncertain. It is also uncertain whether CBT improves general quality of life or negatively biased interpretations of tinnitus.

Compared to audiological care, tinnitus retraining therapy and other types of treatment, there were findings that CBT probably reduces the negative impact of tinnitus on quality of life. The certainty of this evidence ranged from moderate to low. Where reported, there were few adverse effects and no significant differences between the groups. For depression, anxiety and general quality of life the results were more mixed and the evidence less certain. There is moderate-certainty evidence that CBT may reduce negatively biased interpretations of tinnitus compared to other types of treatment, but compared to audiological care and tinnitus retraining therapy the evidence is less certain.

\section{How up to date is this review?}

The review authors searched for studies that had been published up to November 2019. 


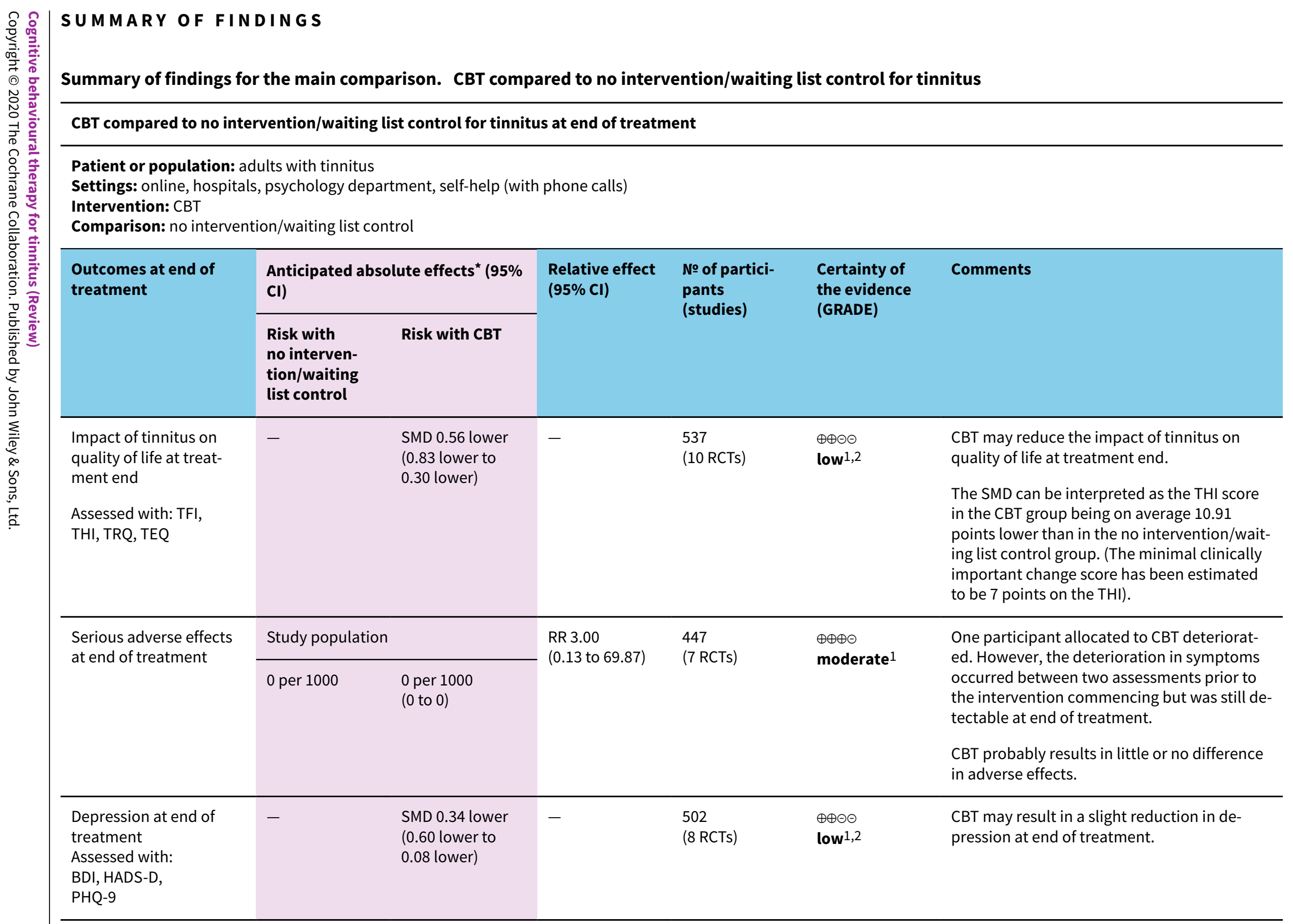

Pent or population: adults with tinnitus

Settings: online, hospitals, psychology department, self-help (with phone calls)

Comparison: no intervention/waiting list contro 


\begin{tabular}{|c|c|c|c|c|c|}
\hline $\begin{array}{l}\text { Anxiety at end of treat- } \\
\text { ment } \\
\text { Assessed with: }\end{array}$ & $\begin{array}{l}\text { SMD } 0.45 \text { lower } \\
\text { (0.82 lower to } \\
0.09 \text { lower) }\end{array}$ & - & $\begin{array}{l}429 \\
(6 \mathrm{RCTs})\end{array}$ & $\begin{array}{l}\oplus \ominus \ominus \ominus \\
\text { very low } 1,3,4\end{array}$ & $\begin{array}{l}\text { The evidence is very uncertain about whether } \\
\text { CBT reduces anxiety at end of treatment. }\end{array}$ \\
\hline $\begin{array}{l}\text { Health-related quality of } \\
\text { life } \\
\text { Assessed with: } \\
\text { SWLS, QoLI }\end{array}$ & $\begin{array}{l}\text { SMD } 0.38 \text { lower } \\
(0.67 \text { lower to } \\
0.08 \text { lower })\end{array}$ & - & $\begin{array}{l}179 \\
(2 \mathrm{RCTs})\end{array}$ & $\begin{array}{l}\oplus \ominus \ominus \ominus \\
\text { very low } 5,6,7\end{array}$ & $\begin{array}{l}\text { The evidence is very uncertain about whether } \\
\text { CBT improves health-related quality of life. }\end{array}$ \\
\hline $\begin{array}{l}\text { Negatively biased inter- } \\
\text { pretations of tinnitus } \\
\text { Assessed with: } \\
\text { TAQ, TCQ }\end{array}$ & $\begin{array}{l}\text { SMD } 0.4 \text { lower } \\
\text { (1.25 lower to } \\
0.45 \text { higher) }\end{array}$ & - & $\begin{array}{l}84 \\
(2 \mathrm{RCTS})\end{array}$ & $\begin{array}{l}\oplus \ominus \ominus \ominus \\
\text { very low } 2,7,8\end{array}$ & $\begin{array}{l}\text { The evidence is very uncertain about whether } \\
\text { CBT reduces negatively biased interpreta- } \\
\text { tions of tinnitus. }\end{array}$ \\
\hline Other adverse effects & No adverse effects occurred. & - & $\begin{array}{l}447 \\
(7 \mathrm{RCTs})\end{array}$ & $\begin{array}{l}\oplus \oplus \oplus \ominus \\
\text { moderate }\end{array}$ & - \\
\hline
\end{tabular}

${ }^{\star}$ The risk in the intervention group (and its $95 \%$ confidence interval) is based on the assumed risk in the comparison group and the relative effect of the intervention (and its $95 \% \mathrm{Cl})$.

BDI: Beck Depression Inventory; CBT: cognitive behavioural therapy; CI: confidence interval; GAD-7: Generalized Anxiety Disorder-7; HADS-A: Hospital Anxiety and Depression Scale-Anxiety; HADS-D: Hospital Anxiety and Depression Scale-Depression; PHQ-9: Patient Health Questionnaire; QoLI: Quality of Life Inventory; RCT: randomised controlled trial; RR: risk ratio; SWLS: Satisfaction With Life Survey; TAQ: Tinnitus Acceptance Questionnaire; TCQ: Tinnitus Cognitions Questionnaire.; TEQ: Tinnitus Effects Questionnaire; TFI: Tinnitus Functional Inventory; THI: Tinnitus Handicap Inventory; TRQ: Tinnitus Reaction Questionnaire

\section{GRADE Working Group grades of evidence}

High certainty: We are very confident that the true effect lies close to that of the estimate of the effect

Moderate certainty: We are moderately confident in the effect estimate: The true effect is likely to be close to the estimate of the effect, but there is a possibility that it is substantially different

Low certainty: Our confidence in the effect estimate is limited: The true effect may be substantially different from the estimate of the effect

Very low certainty: We have very little confidence in the effect estimate: The true effect is likely to be substantially different from the estimate of effect

1Downgraded one level due to study limitations (risk of bias): all studies included for this outcome were judged to be either unclear or at high risk of performance bias due to an absence of blinding of participants and personnel.

2Downgraded one level due to inconsistency: significant heterogeneity in the studies.

${ }^{3}$ Downgraded one level due to study limitations (risk of bias): two studies with a weighting of $36 \%$ were judged to be at high risk of selection bias.

4 Downgraded one level due to inconsistency: confidence intervals of two studies did not overlap and the 12 value was $67 \%$.

5 Downgraded one level due to study limitations (risk of bias): studies judged to be at high risk of selection bias.

${ }^{6}$ Downgraded one level due to study limitations (risk bias): studies judged to be at unclear or high risk of performance and detection bias.

7Downgraded one level due to imprecision: small sample size.

8Downgraded one level due to study limitations (risk of bias): one study judged to be at high risk of selection bias and both were judged to be at uncertain risk of performance and detection biases. 
CBT compared to audiological care (tinnitus education and rehabilitation for hearing loss) for tinnitus at end of treatment

Patient or population: adults with tinnitus

Settings: audiological rehabilitation centre, hospital, Veterans Affairs, online

Intervention: $\mathrm{CBT}$

Comparison: audiological care (tinnitus education and rehabilitation for hearing loss)

\begin{tabular}{|c|c|c|c|c|c|c|}
\hline \multirow{2}{*}{$\begin{array}{l}\text { Outcomes at end of } \\
\text { treatment }\end{array}$} & \multicolumn{2}{|c|}{ Anticipated absolute effects ${ }^{\star}(95 \% \mathrm{CI})$} & \multirow{2}{*}{$\begin{array}{l}\text { Relative effect } \\
(95 \% \mathrm{CI})\end{array}$} & \multirow{2}{*}{$\begin{array}{l}\text { № of partici- } \\
\text { pants } \\
\text { (studies) }\end{array}$} & \multirow{2}{*}{$\begin{array}{l}\text { Certainty of } \\
\text { the evidence } \\
\text { (GRADE) }\end{array}$} & \multirow[t]{2}{*}{ Comments } \\
\hline & $\begin{array}{l}\text { Risk with au- } \\
\text { diological care } \\
\text { (tinnitus edu- } \\
\text { cation and re- } \\
\text { habilitation for } \\
\text { hearing loss) }\end{array}$ & Risk with CBT & & & & \\
\hline $\begin{array}{l}\text { Impact of tinnitus on qual- } \\
\text { ity of life } \\
\text { Assessed with: Tinnitus } \\
\text { Handicap Inventory } \\
\text { Scale from: } 0 \text { to } 100\end{array}$ & 34.14 & $\begin{array}{l}\text { MD } 5.65 \text { lower } \\
\text { (9.79 lower to } 1.5 \\
\text { lower) }\end{array}$ & - & $\begin{array}{l}430 \\
\text { (3 RCTs) }\end{array}$ & $\begin{array}{l}\oplus \oplus \oplus \ominus \\
\text { moderate }^{1}\end{array}$ & $\begin{array}{l}\text { The MD is reported here because the } 3 \\
\text { studies all reported outcome data from } \\
\text { the THI. } \\
\text { CBT probably reduces the impact of tin- } \\
\text { nitus on quality of life when compared } \\
\text { with audiological care. }\end{array}$ \\
\hline Serious adverse effects & \multicolumn{2}{|c|}{ No serious adverse effects occurred. } & - & $\begin{array}{l}410 \\
(2 \mathrm{RCTs})\end{array}$ & $\begin{array}{l}\oplus \oplus \oplus \ominus \\
\text { moderate }^{1}\end{array}$ & $\begin{array}{l}\text { Meta-analysis was not conducted for } \\
\text { this outcome. }\end{array}$ \\
\hline $\begin{array}{l}\text { Depression at end of treat- } \\
\text { ment } \\
\text { Assessed with: HADS-D, } \\
\text { PHQ-9 }\end{array}$ & - & $\begin{array}{l}\text { SMD } 0.18 \text { lower } \\
\text { (0.38 lower to } 0.01 \\
\text { higher) }\end{array}$ & - & $\begin{array}{l}410 \\
\text { (2 RCTs) }\end{array}$ & $\begin{array}{l}\oplus \oplus \ominus \ominus \\
\text { low } 1,2\end{array}$ & $\begin{array}{l}\text { CBT may slightly reduce depression at } \\
\text { end of treatment when compared with } \\
\text { audiological care. }\end{array}$ \\
\hline $\begin{array}{l}\text { Anxiety at end of treat- } \\
\text { ment } \\
\text { Assessed with: GAD-7, } \\
\text { HADS-A }\end{array}$ & - & $\begin{array}{l}\text { SMD } 0.06 \text { lower } \\
\text { (0.26 lower to } 0.13 \\
\text { higher) }\end{array}$ & - & $\begin{array}{l}410 \\
\text { (2 RCTs) }\end{array}$ & $\begin{array}{l}\oplus \oplus \ominus \ominus \\
\text { low } 1,2\end{array}$ & $\begin{array}{l}\text { CBT may result in little to no difference } \\
\text { in anxiety at end of treatment when } \\
\text { compared with audiological care. }\end{array}$ \\
\hline $\begin{array}{l}\text { Health-related quality of } \\
\text { life } \\
\text { Assessed with: HUI, SWLS }\end{array}$ & - & $\begin{array}{l}\text { SMD } 0.07 \text { lower } \\
\text { ( } 0.26 \text { lower to } 0.13 \\
\text { higher) }\end{array}$ & - & $\begin{array}{l}410 \\
\text { (2 RCTs) }\end{array}$ & $\begin{array}{l}\oplus \oplus \ominus \ominus \\
\text { low } 1,2\end{array}$ & $\begin{array}{l}\text { CBT may result in little to no difference } \\
\text { in health-related quality of life when } \\
\text { compared with audiological care. }\end{array}$ \\
\hline
\end{tabular}




\begin{tabular}{|c|c|c|c|c|c|c|}
\hline $\begin{array}{l}\text { Negatively biased inter- } \\
\text { pretations of tinnitus } \\
\text { Assessed with: TCS } \\
\text { Scale from: } 0 \text { to } 65\end{array}$ & $\begin{array}{l}\text { At end of treat- } \\
\text { ment TCS } \\
\text { scores had de- } \\
\text { creased from } \\
\text { a mean of } 21 . \\
42 \text { (SD } 12.56) \\
\text { to } 17.14 \text { (SD } \\
11.54) \text {. }\end{array}$ & $\begin{array}{l}\text { At end of treatment } \\
\text { TCS scores had de- } \\
\text { creased from a mean } \\
\text { of } 20.89 \text { (SD 11.83) to } \\
12.45(10.30) \text {. }\end{array}$ & - & $\begin{array}{l}336 \\
(1 \mathrm{RCT})\end{array}$ & $\begin{array}{l}\oplus \oplus \ominus \ominus \\
\text { low }^{3,4}\end{array}$ & $\begin{array}{l}\text { CBT may reduce negatively biased inter- } \\
\text { pretations of tinnitus when compared } \\
\text { with audiological care. }\end{array}$ \\
\hline Other adverse effects & No adverse effe & s occurred. & - & $\begin{array}{l}410 \\
\text { (2 RCTs) }\end{array}$ & $\begin{array}{l}\oplus \oplus \oplus \ominus \\
\text { moderate }^{1}\end{array}$ & - \\
\hline \multicolumn{7}{|c|}{$\begin{array}{l}\text { *The risk in the intervention group (and its } 95 \% \text { confidence interval) is based on the assumed risk in the comparison group and the relative effect of the intervention (and } \\
\text { its } 95 \% \mathrm{CI} \text { ). The assumed risk score in the comparison group (34.14) was obtained from the median control group score from the largest study (Cima 2012) in this compari- } \\
\text { son. } \\
\text { CBT: cognitive behavioural therapy; CI: confidence interval; GAD-7: Generalized Anxiety Disorder-7; HADS-A: Hospital Anxiety and Depression Scale-Anxiety; HADS-D: Hos- } \\
\text { pital Anxiety and Depression Scale-Depression; HUI: Health Utilities Index; MD: mean difference; PHQ-9: Patient Health Questionnaire; RCT: randomised controlled trial; } \\
\text { RR: risk ratio; SWLS: Satisfaction With Life Survey; TCS: Tinnitus Catastrophizing Scale }\end{array}$} \\
\hline \multicolumn{7}{|c|}{$\begin{array}{l}\text { GRADE Working Group grades of evidence } \\
\text { High certainty: We are very confident that the true effect lies close to that of the estimate of the effect } \\
\text { Moderate certainty: We are moderately confident in the effect estimate: The true effect is likely to be close to the estimate of the effect, but there is a possibility that it is } \\
\text { substantially different } \\
\text { Low certainty: Our confidence in the effect estimate is limited: The true effect may be substantially different from the estimate of the effect } \\
\text { Very low certainty: We have very little confidence in the effect estimate: The true effect is likely to be substantially different from the estimate of effect }\end{array}$} \\
\hline \multicolumn{7}{|c|}{$\begin{array}{l}\text { 1Downgraded one level due to study limitations (risk of bias): all studies included for this outcome were judged to be either unclear or at high risk of performance bias due to } \\
\text { an absence of blinding of participants and personnel. } \\
\text { 2Downgraded by one level due to imprecision: the confidence intervals cross the line of no effect. } \\
\text { 3Downgraded one level due to study limitations (risk of bias): performance and detection bias judged as unclear. } \\
\text { 4Downgraded one level due to imprecision: small sample size. }\end{array}$} \\
\hline \multicolumn{7}{|c|}{ Summary of findings 3. CBT compared to TRT (directive counselling and bilateral masking) for tinnitus } \\
\hline \multicolumn{7}{|c|}{ CBT compared to TRT (directive counselling and bilateral masking) for tinnitus at end of treatment } \\
\hline \multicolumn{7}{|c|}{$\begin{array}{l}\text { Patient or population: adults with tinnitus } \\
\text { Setting: hospital } \\
\text { Intervention: CBT } \\
\text { Comparison: TRT (directive counselling and bi }\end{array}$} \\
\hline
\end{tabular}




\begin{tabular}{|c|c|c|c|c|c|c|}
\hline \multirow{2}{*}{ 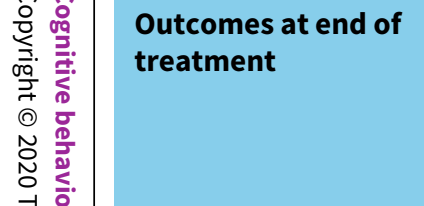 } & \multicolumn{2}{|c|}{ Anticipated absolute effects ${ }^{*}(95 \% \mathrm{Cl})$} & \multirow{2}{*}{$\begin{array}{l}\text { Relative effect } \\
(95 \% \mathrm{Cl})\end{array}$} & \multirow{2}{*}{$\begin{array}{l}\text { № of partici- } \\
\text { pants } \\
\text { (studies) }\end{array}$} & \multirow{2}{*}{$\begin{array}{l}\text { Certainty of } \\
\text { the evidence } \\
\text { (GRADE) }\end{array}$} & \multirow[t]{2}{*}{ Comments } \\
\hline & $\begin{array}{l}\text { Risk with TRT (directive } \\
\text { counselling and bilateral } \\
\text { masking) }\end{array}$ & Risk with CBT & & & & \\
\hline $\begin{array}{l}\text { Impact of tinnitus on } \\
\text { quality of life } \\
\text { Assessed with: THI } \\
\text { Scale from: } 0 \text { to } 100\end{array}$ & $\begin{array}{l}\text { At } 10 \text { weeks the THI score } \\
\text { had decreased from an av- } \\
\text { erage of } 47.00 \text { (SD } 18.19 \text { ) } \\
\text { to an average of } 43.22 \text { (SD } \\
20.75 \text { ). }\end{array}$ & $\begin{array}{l}\text { At } 10 \text { weeks the THI score } \\
\text { had decreased from an av- } \\
\text { erage of } 45.27 \text { (SD 14.99) } \\
\text { to an average of } 27.43 \\
\text { (19.18). }\end{array}$ & - & $\begin{array}{l}42 \\
(1 \mathrm{RCT})\end{array}$ & $\begin{array}{l}\oplus \oplus \ominus \ominus \\
\text { low } 1,2\end{array}$ & $\begin{array}{l}\text { CBT may reduce the impact } \\
\text { of tinnitus on quality of life } \\
\text { compared with TRT. }\end{array}$ \\
\hline \multirow{2}{*}{$\begin{array}{l}\text { Serious adverse ef- } \\
\text { fects }\end{array}$} & \multicolumn{2}{|l|}{ Study population } & \multirow{2}{*}{$\begin{array}{l}\text { RR } 0.45 \\
(0.04 \text { to } 4.64)\end{array}$} & \multirow{2}{*}{$\begin{array}{l}42 \\
(1 \mathrm{RCT})\end{array}$} & \multirow{2}{*}{$\begin{array}{l}\oplus \oplus \ominus \ominus \\
\text { low }^{1,2}\end{array}$} & \multirow{2}{*}{$\begin{array}{l}\text { Three participants deteri- } \\
\text { orated over the course of } \\
\text { the study: } 1 \text { participant was } \\
\text { from the intervention group } \\
(A C T ; n=22 \text { ) and } 2 \text { partici- } \\
\text { pants were from the com- } \\
\text { parison group (TRT; } n=20 \text { ). }\end{array}$} \\
\hline & 100 per 1000 & $\begin{array}{l}45 \text { per } 1000 \\
(4 \text { to } 464)\end{array}$ & & & & \\
\hline $\begin{array}{l}\text { Depression } \\
\text { Assessed with: } \\
\text { HADS-D } \\
\text { Scale from: } 0 \text { to } 21\end{array}$ & $\begin{array}{l}\text { At } 10 \text { weeks the HADS-D } \\
\text { scores had decreased from } \\
\text { a mean of } 5.80 \text { (SD 3.79) to } \\
5.78 \text { (SD 3.73). }\end{array}$ & $\begin{array}{l}\text { At } 10 \text { weeks the HADS- } \\
\text { D scores had decreased } \\
\text { from a mean of } 4.05 \text { (SD } \\
3.06 \text { ) to } 3.20 \text { (SD } 3.47 \text { ). }\end{array}$ & - & $\begin{array}{l}42 \\
(1 \mathrm{RCT})\end{array}$ & $\begin{array}{l}\oplus \oplus \ominus \ominus \\
\text { low } 1,2\end{array}$ & $\begin{array}{l}\text { We are uncertain whether } \\
\text { CBT reduces depression } \\
\text { compared with TRT. }\end{array}$ \\
\hline $\begin{array}{l}\text { Anxiety } \\
\text { Assessed with: } \\
\text { HADS-A } \\
\text { Scale from: } 0 \text { to } 21\end{array}$ & $\begin{array}{l}\text { At } 10 \text { weeks the HADS-A } \\
\text { scores had decreased from } \\
\text { a mean of } 8.2 \text { (SD 3.75) to } \\
7.0 \text { (SD 4.20). }\end{array}$ & $\begin{array}{l}\text { At } 10 \text { weeks the HADS- } \\
\text { A scores had decreased } \\
\text { from a mean of } 6.24 \text { (SD } \\
4.00 \text { ) to } 3.6 \text { (SD 3.14). }\end{array}$ & - & $\begin{array}{l}42 \\
(1 \mathrm{RCT})\end{array}$ & $\begin{array}{l}\oplus \oplus \ominus \ominus \\
\text { low } 1,2\end{array}$ & $\begin{array}{l}\text { We are uncertain whether } \\
\text { CBT reduces anxiety com- } \\
\text { pared with TRT. }\end{array}$ \\
\hline $\begin{array}{l}\text { Health-related quali- } \\
\text { ty of life } \\
\text { Assessed with: QoLI } \\
\text { Scale from: }-6 \text { to } 6\end{array}$ & $\begin{array}{l}\text { At } 10 \text { weeks QoLI scores had } \\
\text { increased from a mean of } \\
2.24 \text { (SD 1.42) to } 2.47 \text { (SD } \\
1.72 \text { ). }\end{array}$ & $\begin{array}{l}\text { At } 10 \text { weeks QoLI scores } \\
\text { had increased from a } \\
\text { mean of } 2.43 \text { (SD } 1.30 \text { ) to } \\
2.78 \text { (SD 1.53). }\end{array}$ & - & $\begin{array}{l}42 \\
(1 \mathrm{RCT})\end{array}$ & $\begin{array}{l}\oplus \oplus \ominus \ominus \\
\text { low } 1,2\end{array}$ & $\begin{array}{l}\text { We are uncertain whether } \\
\text { CBT improves health-relat- } \\
\text { ed quality of life compared } \\
\text { with TRT. }\end{array}$ \\
\hline $\begin{array}{l}\text { Negatively biased in- } \\
\text { terpretations of tin- } \\
\text { nitus } \\
\text { Assessed with: TAQ } \\
\text { Scale from: } 0 \text { to } 72\end{array}$ & $\begin{array}{l}\text { At } 10 \text { weeks TAQ scores had } \\
\text { increased from a mean of } \\
36.65(9.96) \text { to } 37.89 \text { (SD } \\
10.73) \text {. }\end{array}$ & $\begin{array}{l}\text { At } 10 \text { weeks TAQ scores } \\
\text { had increased from a } \\
\text { mean of } 41.05 \text { (SD 9.49) to } \\
47.67 \text { (SD 11.15). }\end{array}$ & - & $\begin{array}{l}42 \\
(1 \mathrm{RCT})\end{array}$ & $\begin{array}{l}\oplus \oplus \ominus \ominus \\
\text { low } 1,2\end{array}$ & $\begin{array}{l}\text { CBT may reduce negative- } \\
\text { ly biased interpretations of } \\
\text { tinnitus compared with TRT. }\end{array}$ \\
\hline Other adverse effects & \multicolumn{2}{|c|}{ No other adverse effects were reported. } & - & $\begin{array}{l}42 \\
(1 \mathrm{RCT})\end{array}$ & $\begin{array}{l}\oplus \oplus \ominus \ominus \\
\text { low } 1,2\end{array}$ & - \\
\hline
\end{tabular}


${ }^{*}$ The risk in the intervention group (and its $95 \%$ confidence interval) is based on the assumed risk in the comparison group and the relative effect of the intervention (and its $95 \% \mathrm{Cl})$.

ACT: acceptance and commitment therapy; Cl: confidence interval; HADS-A: Hospital Anxiety and Depression Scale-Anxiety; HADS-D: Hospital Anxiety and Depression Scale-Depression; QoLI: Quality of Life Inventory; RCT: randomised controlled trial; RR: risk ratio; SD: standard deviation; TAQ: Tinnitus Acceptance Questionnaire; THI: Tinnitus Handicap Inventory; TRT: tinnitus retraining therapy

\section{GRADE Working Group grades of evidence}

High certainty: We are very confident that the true effect lies close to that of the estimate of the effect

Moderate certainty: We are moderately confident in the effect estimate: The true effect is likely to be close to the estimate of the effect, but there is a possibility that it is substantially different

Low certainty: Our confidence in the effect estimate is limited: The true effect may be substantially different from the estimate of the effect

Very low certainty: We have very little confidence in the effect estimate: The true effect is likely to be substantially different from the estimate of effect

1Downgraded one level due to study limitations (risk of bias). There was high risk of bias associated with allocation concealment and unclear risk of bias for performance, detection and attrition bias respectively.

2Downgraded one level due to imprecision: small sample size.

\section{Summary of findings 4. CBT compared to other experimental control for tinnitus}

\section{CBT compared to other experimental control for tinnitus}

Patient or population: adults with tinnitus

Settings: hospital, online

Intervention: CBT

Comparison: other experimental control

\begin{tabular}{|c|c|c|c|c|c|c|}
\hline \multirow[t]{2}{*}{$\begin{array}{l}\text { Outcomes at end of } \\
\text { treatment }\end{array}$} & \multicolumn{2}{|c|}{$\begin{array}{l}\text { Anticipated absolute effects }{ }^{\star} \text { ( } 95 \% \\
\mathrm{Cl})\end{array}$} & \multirow[t]{2}{*}{$\begin{array}{l}\text { Relative effect } \\
(95 \% \mathrm{CI})\end{array}$} & \multirow{2}{*}{$\begin{array}{l}\text { № of partici- } \\
\text { pants } \\
\text { (studies) }\end{array}$} & \multirow{2}{*}{$\begin{array}{l}\text { Certainty of } \\
\text { the evidence } \\
\text { (GRADE) }\end{array}$} & \multirow[t]{2}{*}{ Comments } \\
\hline & $\begin{array}{l}\text { Risk with other } \\
\text { experimental } \\
\text { control }\end{array}$ & Risk with CBT & & & & \\
\hline $\begin{array}{l}\text { Impact of tinnitus on qual- } \\
\text { ity of life at end of treat- } \\
\text { ment } \\
\text { Assessed with: TFI, THI, } \\
\text { THQ, TQ, TRQ, TEQ-ED }\end{array}$ & - & $\begin{array}{l}\text { SMD } 0.30 \text { lower } \\
\text { (0.55 lower to } 0.05 \\
\text { lower) }\end{array}$ & - & $\begin{array}{l}966 \\
(12 \text { RCTs })\end{array}$ & $\begin{array}{l}\oplus \oplus \ominus \ominus \\
\text { low } 1,2\end{array}$ & $\begin{array}{l}\text { CBT may reduce the impact of tinnitus on } \\
\text { quality of life when compared with other } \\
\text { treatments. } \\
\text { The SMD can be interpreted as the THI } \\
\text { score in the CBT group being on average } \\
5.84 \text { points lower than in the other exper- } \\
\text { imental control group. (The minimal clini- }\end{array}$ \\
\hline
\end{tabular}




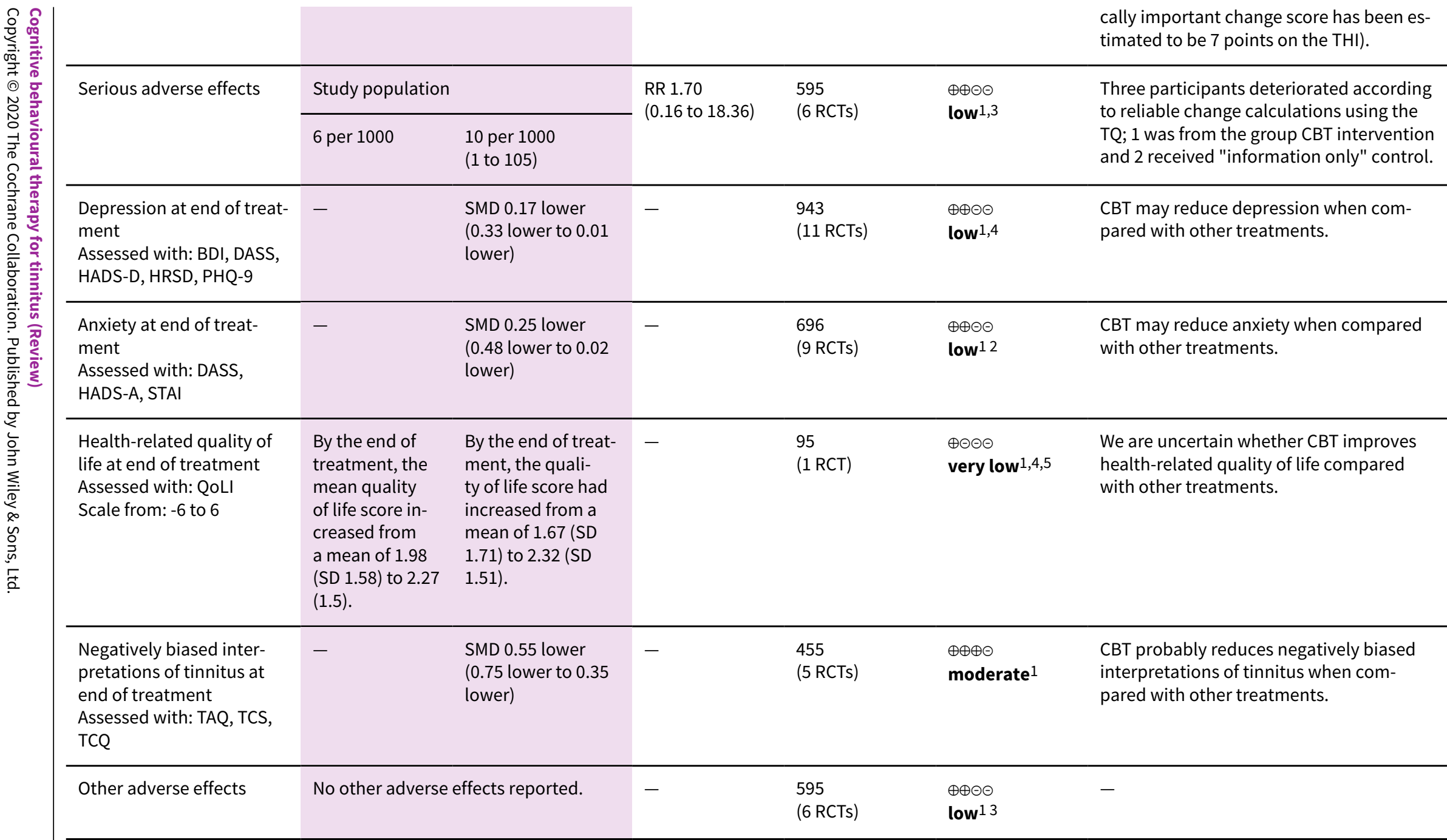

*The risk in the intervention group (and its $95 \%$ confidence interval) is based on the assumed risk in the comparison group and the relative effect of the intervention (and its $95 \% \mathrm{Cl})$.

BDI: Beck Depression Inventory; CI: confidence interval; DASS: Depression Anxiety and Stress Scale; GAD-7: Generalized Anxiety Disorder-7; HADS-A: Hospital Anxiety and Depression Scale-Anxiety; HADS-D: Hospital Anxiety and Depression Scale-Depression; HRSD: Hamilton Rating Scale for Depression; PHQ-9: Patient Health Questionnaire; QoLI: Quality of Life Inventory; RR: risk ratio; STAI: State Trait Anxiety Inventory; TAQ: Tinnitus Acceptance Questionnaire; TCQ: Tinnitus Cognitions Questionnaire; TCS: Tinnitus Catastrophizing Scale; TEQ-ED: Tinnitus Effects Questionnaire-Emotional Distress; TFI: Tinnitus Functional Inventory; THI: Tinnitus Handicap Inventory; THQ: Tinnitus Handicap Questionnaire; TQ: Tinnitus Questionnaire; TRQ: Tinnitus Reaction Questionnaire 
GRADE Working Group grades of evidence

High certainty: We are very confident that the true effect lies close to that of the estimate of the effect

Moderate certainty: We are moderately confident in the effect estimate: The true effect is likely to be close to the estimate of the effect, but there is a possibility that it is substantially different

Low certainty: Our confidence in the effect estimate is limited: The true effect may be substantially different from the estimate of the effect

Very low certainty: We have very little confidence in the effect estimate: The true effect is likely to be substantially different from the estimate of effect

1Downgraded one level due to study limitations (risk of bias): all studies included for this outcome were judged to be either unclear or at high risk of performance bias due to an absence of blinding of participants and personnel.

2Downgraded one level due to inconsistency: not all the confidence intervals overlap, and statistical heterogeneity is relatively high and statistically significant

${ }^{3}$ Downgraded one level due to study limitations (risk of bias): one study judged to be at high risk and all others, except one, at unclear risk of attrition bias.

4Downgraded one level due to imprecision: overall confidence interval crosses the line of no effect.

5Downgraded one level due to imprecision: small sample size. 


\section{B A C K G R O U N D}

The following paragraphs and Description of the condition are based on the Cochrane Review 'Amplification with hearing aids for patients with tinnitus and co-existing hearing loss' and reproduced with permission (Hoare 2014).

Tinnitus is defined as the perception of sound in the absence of a corresponding auditory source (Jastreboff 2004). It is typically described by those who experience it as a ringing, hissing, buzzing or whooshing sound and is thought to result from abnormal neural activity and connectivity in auditory and non-auditory pathways, which is interpreted by the brain as sound (Elgoyhen 2015; Shore 2016). Tinnitus can be either objective or subjective.

Objective tinnitus is estimated to occur in up to $10 \%$ of people with tinnitus seeking help (Kircher 2008), and refers to the perception of sound that can also be heard by the examiner (Roberts 2010). Objective forms include heartbeat synchronous pulsatile tinnitus and they usually have a detectable cause such as arteriovenous malformation, carotid stenosis or dissections (Langguth 2013). Specific medication or surgical treatment can lead to the cessation of the objective tinnitus percept (Kleinjung 2016).

Most commonly, however, tinnitus is subjective, meaning that the sound is only heard by the person experiencing it and no source of the sound can be identified (Jastreboff 1988). Subjective tinnitus (the focus of this review) is estimated to affect up to $21 \%$ of the general adult population, increasing to as many as $30 \%$ of adults over 50 years of age (Davis 2000; Gallus 2015; Kim 2015). It can be experienced acutely, recovering spontaneously within minutes to weeks. However, it can become chronic and is unlikely to resolve spontaneously when experienced for three months or more (Hahn 2008; Hall 2011; Rief 2005). In 1\% to $3 \%$ of the population tinnitus causes severe problems with daily life functioning (Davis 2000; Kim 2015). Although a range of psychological, sound, electrical and electromagnetic therapies have been developed, currently there is no reliable cure for subjective tinnitus.

In England alone there are an estimated 3/4 million General Practitioner consultations every year where the primary complaint is tinnitus (El-Shunnar 2011), equating to a major burden on healthcare services. For many people tinnitus is persistent and troublesome, and has disabling effects such as insomnia, difficulty concentrating, difficulties in communication and social interaction, and negative emotional responses such as anxiety and depression (Andersson 2009; Cima 2011b; Crönlein 2007; Langguth 2011; Marciano 2003; Zirke 2013a; Zirke 2013b). In approximately $90 \%$ of cases, chronic tinnitus is co-morbid with some degree of hearing loss, which may confound these disabling effects (Fowler 1944; Sanchez 2002). An important implication of this in clinical research is that outcome measures need to distinguish benefits specific to the tinnitus signal itself and related aspects such as impairments in communication, emotional processing and social interaction, which all play a relevant role in quality of life.

For the purposes of this review we will use 'the impact of tinnitus on quality of life' (or tinnitus-related quality of life) as a collective term for the cognitive, emotional and behavioural consequences/ sequelae that people living with chronic tinnitus experience. Additionally, unless otherwise noted, we will refer to subjective tinnitus simply as tinnitus.

\section{Description of the condition}

\section{Pathophysiology}

Most people with chronic tinnitus have some degree of hearing loss (Ratnayake 2009), and the prevalence of tinnitus increases with greater hearing loss (Han 2009; Martines 2010). Converging evidence from animal models and studies of human tinnitus sufferers indicates that, while cochlear damage is a trigger, most cases of tinnitus are generated by changes that take place in central auditory pathways when auditory neurons lose their input from the ear (Noreña 2011). Forms of neural plasticity underlie these neural changes, which include: increased spontaneous activity and neural gain in deafferented central auditory structures; increased synchronous activity in these structures; and changes in network behaviour in non-auditory brain regions. These changes have been detected by functional imaging of individuals with tinnitus and corroborated by animal investigations (Eggermont 2014; Elgoyhen 2015). (Additional detail is provided in Appendix 1).

A complication in understanding the pathophysiology of tinnitus is that not all people with hearing loss have tinnitus and not all people with tinnitus have a clinically significant hearing loss. Other variables, such as the profile of a person's hearing loss, may account for differences in their tinnitus report. For example, König 2006 found that the maximum slope within audiograms was higher in people with tinnitus than in people with hearing loss who do not have tinnitus, despite the 'non-tinnitus' group having the greater mean hearing loss. Also the additional involvement of non-auditory areas of the brain, particularly areas associated with awareness and salience detection, can explain why some people with hearing loss develop tinnitus whereas others do not (de Ridder 2011; de Ridder 2014).

Whether tinnitus is perceived as bothersome or not may be related to the additional involvement of emotion processing areas (Rauschecker 2010; Schecklmann 2013; Vanneste 2012). Accordingly, some models have proposed that tinnitus reflects "an emergent property of multiple parallel dynamically changing and partially overlapping sub-networks". This suggests that various brain networks associated with memory and emotional processing are involved in tinnitus and that the degree of involvement of the different networks reflects the variable aspects of an individual's tinnitus (de Ridder 2011; de Ridder 2014; Elgoyhen 2015).

\section{Psychological models of tinnitus}

In addition to the physiological data and models of tinnitus, psychological models have been developed to explain how and why some people experience a negative impact of tinnitus on quality of life. Psychological models of tinnitus include those developed by Hallam, which applies the concept of habituation (Hallam 1984); Jastreboff, whose model features classical conditioning mechanisms (Jastreboff 1988; Jastreboff 1990); and the cognitive behavioural models of McKenna 2014, Cima 2011b and Kleinstauber 2013 (Appendix 2). These psychological models underpin the rationale and development of cognitive behavioural interventions for reducing the impact of tinnitus on quality of life.

\section{Diagnosis and clinical management of tinnitus}

There is no universal internationally established standard procedure for the diagnosis or management of tinnitus. However, common across the (few) published practice guidelines is the use 
or recommendation of self-report questionnaires to assess tinnitus and its impact on patients by measuring severity, quality of life, depression or anxiety (Fuller 2017a). Psychoacoustic measures of tinnitus (pitch, loudness, minimum masking level) are also used in patient assessment but do not correlate well with selfreported measures of tinnitus annoyance (Hiller 2006). Instead they represent measurements of tinnitus that can be useful in patient counselling by, for example, demonstrating changes (or stability) in the individual's perception of the tinnitus over time (Department of Health 2009). No objective measures of tinnitus currently exist and so researchers and clinicians are reliant upon patient selfreport measures (usually questionnaires with Likert-type or visual analogue scales) to record any changes in tinnitus related quality of life or other general health effects of therapy (Appendix 3). The previous Cochrane Review of cognitive behavioural therapy for tinnitus used self-reported, subjective tinnitus loudness as the primary outcome measure (Martinez-Devesa 2010). That review and others like it have consistently reported that there are generally weak (if any) effects of the intervention on the level of perceived loudness of the tinnitus (Andersson 1999; Martinez-Devesa 2010). Additionally, concerns have been raised about what is actually being measured when people are asked to rate the subjective loudness of their tinnitus (McKenna 2014).

Clinical management strategies include education and/or counselling, relaxation therapy, tinnitus retraining therapy (TRT), cognitive behavioural therapies (CBT) and sound enrichment using ear-level sound generators or hearing aids (Henry 2005). In addition, electrical and neurostimulation, as well as drug therapies aimed at treating tinnitus directly, or managing comorbid symptoms such as insomnia, anxiety or depression, have been tested. The effects of these management options are variable, they have inconclusive outcomes and some have risks or adverse effects (Dobie 1999; Hoare 2011a; Hoare 2011b; Hobson 2012; Langguth 2013; Martinez-Devesa 2010; Phillips 2010).

\section{Description of the intervention}

Cognitive behavioural therapy (CBT) is an inclusive term that features and combines numerous psychological interventions that were developed and evolved from cognitive and behavioural therapies respectively. CBT for tinnitus aims primarily to reduce the impact of tinnitus on quality of life, rather than directly change the perceived loudness.

Behavioural therapies (e.g. behavioural activation, exposure, relaxation) aim to help patients overrule learned associations between tinnitus and counter-productive responses (e.g. avoiding tinnitus-increasing activities). Cognitive therapies, on the other hand, focus on the relationship between thoughts and emotions (Ellis 1977), and apply a process of identification and modification of errors in thought processing of experiences (Beck 1979). Combined, the behavioural and cognitive theories have produced a range of intervention components designed to address the dysfunctional thought processes, behavioural and emotional responses that maintain low tinnitus-related quality of life..

As discussed by Cima 2014, cognitive behavioural interventions such as mindfulness-based stress reduction (also known as 'mindfulness'; Kabat-Zinn 1982) and acceptance and commitment therapy (ACT; Hayes 1999) have been developed and applied to the treatment of the impact of tinnitus on quality of life (e.g. Hesser 2009; Philippot 2012). For the purposes of this review, we will not make distinctions between whether an intervention is 'first', 'second' or 'third wave' CBT. Instead, we will treat $A C T$ and mindfulness interventions as CBT and in the course of data extraction we will identify components/elements within all interventions as behavioural, cognitive or a combination (i.e. CBT).

Interventions described or labelled as 'CBT' cannot be assumed to be equivalent homogenous entities. Even if $\mathrm{CBT}$ interventions comprise the same elements they might vary with regard to: the mode of delivery of the intervention (e.g. face-to-face, mediated via telephone, Internet); the frequency of sessions (e.g. daily, weekly, fortnightly); the length of sessions; the duration of the intervention; who delivers the CBT (e.g. psychologist, social worker, nurse, computer program); the setting in which the treatment is delivered (e.g. hospital, health centre, private clinic); and whether the therapy is delivered in a group or individual format.

The previous Cochrane Review of CBT for tinnitus found that there were no reported adverse effects in the included studies (Martinez-Devesa 2010). It is, however, conceivable that people might experience a deterioration in their mood during the course of CBT, due to the often challenging nature of the therapy or the distress arising as a result of changes in cognitive and emotional mechanisms. It is also possible that adverse effects were not reported by the authors of studies included in the review, as this is a common occurrence in studies (Pitrou 2009).

\section{How the intervention might work}

Since a growing body of evidence suggests that the impact of tinnitus on quality of life depends more on psychological factors than acoustic properties (Cima 2014; Milerova 2013), psychological therapies have been widely used for tinnitus treatment.

Cognitive strategies are based on the idea that negatively biased interpretations or thoughts about specific events or experiences, such as hearing tinnitus, produce a dysfunctional emotional and/or behavioural response (Beck 1979; Ellis 1977). Thus, cognitive strategies are thought to work by identifying any biased or irrational thinking styles (such as catastrophising), then challenging, modifying and/or replacing them with alternative and more realistic beliefs that lead to a more adaptive response.

A behavioural intervention such as an exposure therapy might be utilised to decrease the impact of tinnitus on daily life. Exposure to the tinnitus sound is thought to work through a process of extinction learning and generalisation. That is, a person learns that the tinnitus sound is no longer indicative of being emotionally aroused or in a distressed state and applies this new knowledge to situations beyond those learned in the therapeutic setting. In daily life this might mean a person re-engages in activities that they previously avoided for fear that the tinnitus would deteriorate.

Individually, cognitive and behavioural therapy components are hypothesised to have specific effects. For example, education regarding the physiology and pathophysiology of hearing and tinnitus are thought to provide a foundation on which patients can begin to understand that tinnitus is not a harmful symptom in its own right and hence nothing, logically at least, to be afraid of. Cognitive behavioural approaches to tinnitus therapy are therefore hypothesised to affect a reduction in impact of tinnitus on quality of life through the summed or synergistic effects of the specific intervention components included in an individual 
therapy. Further, it is hypothesised that this has a consequent effect of reducing generalised anxiety or depression where it is co-morbid, and generally improving self-reported quality of life.

To date there has been little detailed research examining precisely when therapeutic change occurs during the course of CBT treatments, but they have been reported to be effective over at least a 12-month period (e.g. Cima 2012).

\section{Why it is important to do this review}

This review includes recent randomised controlled trials of CBT for tinnitus that were not included in previous meta-analyses or recent reviews. The most recently published review of CBT interventions for tinnitus was a historical and narrative overview in which a range of study designs in addition to RCTs were included, but also one in which neither a risk of bias assessment was undertaken nor a meta-analysis conducted (Cima 2014). These methodological issues make it harder to draw conclusions about the strength of any treatment effects and risks of bias in the evidence included in the narrative synthesis.

A second reason is that it was also important to address new questions that will inform decisions about service provision, as this has particular relevance for the policy-makers and agencies involved in the funding of treatment (e.g. insurance companies). CBT for tinnitus is generally well received by patients and is potentially a cost-effective means for reducing the impact of tinnitus on quality of life (Maes 2014), but it would also be informative to compare the effectiveness of CBT delivered in group and individual formats and CBT performed by psychologists compared with other health professionals.

Finally, since the previous version of the Cochrane Review of CBT for tinnitus was published (Martinez-Devesa 2010), Cochrane standards for the conduct of intervention reviews have been revised (Higgins 2013; Higgins 2016). This new review not only includes recent randomised controlled trials, but also complies with the new standards.

In summary, this review synthesises the latest evidence related to CBT for tinnitus, which will help inform decisions on whether CBT for tinnitus is effective at reducing the impact of tinnitus on quality of life.

\section{OB JECTIVES}

To assess the effects and safety of CBT for tinnitus in adults.

\section{METHODS}

\section{Criteria for considering studies for this review}

\section{Types of studies}

Randomised controlled trials (including cluster-randomised). If included studies had used a cross-over design, we would have only included data from the first treatment phase. Quasi-randomised controlled studies were not included.

We did not apply restrictions on language, year of publication or publication status.

\section{Types of participants}

Participants were at least 18 years of age with tinnitus as the primary reason for seeking treatment.

In the event that studies included an age range of participants below 18 years (e.g. 16 to 21 years), they were included if the mean age was 18 years or above.

\section{Types of interventions}

The primary intervention of interest was CBT. For the purposes of this review we included studies that also described CBT interventions that apparently only used cognitive or behavioural elements. Interventions such as acceptance and commitment therapy (ACT) and mindfulness were also included but simply considered as types of CBT.

We considered interventions as 'mindfulness' if they involved: exercises that involved self-regulation of attention on experience and emphasised openness, curiosity and acceptance (Bishop 2004).

For the purposes of determining similarities for subgroup analysis, we would have attempted to contact authors of studies that examined the effectiveness of an apparently 'pure' cognitive or behavioural interventions to obtain treatment manuals or protocols.

Upon receipt of any protocols, two authors would have then independently reviewed the intervention manual classifying treatment elements as either cognitive or behavioural. Based on results from a review of treatment components used in psychological therapy for people with tinnitus (Thompson 2016) and the behaviour change taxonomy (Michie 2013), we classified interventions as either 'cognitive only', 'behavioural only' or 'CBT'. In the event that the review authors had differed in their judgements, a third review author would have acted as an arbiter.

We stratified studies into four comparisons:

- $\mathrm{CBT}$ versus no intervention/waiting list control;

- CBT versus usual audiological care (tinnitus education and rehabilitation for hearing loss);

- CBT versus TRT (directive counselling and the use of bilateral sound generators as per TRT protocol);

- CBT versus other experimental control (pooled if using the same experimental control). Other experimental controls may include transcranial magnetic stimulation, electrical or electromagnetic stimulation therapy and bio- neuro-feedback.

\section{Types of outcome measures}

We analysed the following outcomes in the review, but did not use them as a basis for including or excluding studies.

\section{Primary outcomes}

- Impact of tinnitus on quality of life as measured by validated tinnitus-specific multi-item questionnaires identified in a systematic review of outcome instruments used in studies of interventions for tinnitus (Hall 2016). These included:

○ Tinnitus Functional Index;

- Tinnitus Handicap Inventory;

- Tinnitus Handicap Questionnaire; 
- Tinnitus Questionnaire;

- Tinnitus Reaction Questionnaire;

- Tinnitus Disability Index;

- Tinnitus Severity Scale.

For references associated with the outcome measures see Appendix 4).

If a study used multiple measures of the impact of tinnitus on quality of life we applied the following as a hierarchy of the outcome measures based on their known psychometric validity (Fackrell 2014): Tinnitus Functional Index, Tinnitus Handicap Inventory, Tinnitus Handicap Questionnaire, Tinnitus Questionnaire, Tinnitus Reaction Questionnaire, Tinnitus Disability Index, Tinnitus Severity Scale and then other tinnitus-specific questionnaires. Invariably these questionnaires show good convergent validity.

- Serious adverse effects: self-harm, suicide, suicide attempt, suicidal crisis, severe symptom exacerbation.

\section{Secondary outcomes}

- Generalised depression as measured by validated questionnaires, such as the Beck Depression Inventory II (Beck 1996), the depression scale of the Hospital Anxiety and Depression Scale (HADS; Zigmond 1983), and the Hamilton Rating Scale for Depression (Hamilton 1960).

- Generalised anxiety as measured by a validated scale, for example, the anxiety scale of the HADS or Beck Anxiety Inventory (Beck 1988) or the Anxiety Sensitivity Index (Reiss 1986).

- Health-related quality of life as measured by a validated scale, for example, the Short-Form 36 (Hays 1993), WHOQoL-BREF (Skevington 2004), and other WHOQoL versions, Health Utilities Index (Furlong 2001).

- Negatively biased interpretations of tinnitus as measured by a validated scale, such as the Tinnitus Catastrophizing Scale (Cima 2011b), the Fear of Tinnitus Questionnaire (Cima 2011b), and the Tinnitus Fear and Avoidance Scale (Kleinstauber 2013).

- Other adverse effects: acute emotional discomfort.

We measured outcomes at treatment end (typically six to eight weeks) and at long-term follow-up (6 and 12 months).

\section{Search methods for identification of studies}

The Cochrane ENT Information Specialist conducted systematic searches for randomised controlled trials and controlled clinical trials. There were no language, publication year or publication status restrictions. The date of the search was 25 November 2019.

\section{Electronic searches}

The Information Specialist searched:

- the Cochrane ENT Trials Register (searched 25 November 2019);
- CENTRAL (2019, Issue 11) via the Cochrane Register of Studies (25 November 2019);

- Epub Ahead of Print, In-Process \& Other Non-Indexed Citations, Ovid MEDLINE(R) Daily and Ovid MEDLINE(R) 1946 to 25 November 2019);

- Ovid EMBASE (1974 to 25 November 2019);

- EBSCO CINAHL (1982 to 25 November 2019);

- Ovid AMED (1985 to 25 November 2019);

- Ovid PsycINFO (1806 to 25 November 2019);

- Web of Knowledge, Core Collection (1945 to 25 November 2019);

- ClinicalTrials.gov, (searched via the Cochrane Register of Studies 26 November 2019);

- World Health Organization (WHO) International Clinical Trials Registry Platform (ICTRP), www.who.int/ictrp (searched via the Cochrane Register of Studies 26 November 2019).

In searches prior to November 2019, we also searched LILACS, KoreaMed, IndMed and PakMediNet to November 2018.

The Information Specialist modelled subject strategies for databases on the search strategy designed for CENTRAL. Where appropriate, they were combined with subject strategy adaptations of the highly sensitive search strategy designed by Cochrane for identifying randomised controlled trials and controlled clinical trials (as described in the Cochrane Handbook for Systematic Reviews of Interventions Version 5.1.0, Box 6.4.b. (Higgins 2011). Search strategies for major databases including CENTRAL are provided in Appendix 5.

\section{Searching other resources}

We scanned the reference lists of identified publications for additional studies and contacted study authors where necessary. In addition, the Information Specialist searched Ovid MEDLINE to retrieve existing systematic reviews relevant to this systematic review, so that we could scan their reference lists for additional studies. The Information Specialist also ran non-systematic searches of Google Scholar to retrieve grey literature and other sources of potential studies.

\section{Data collection and analysis}

\section{Selection of studies}

Thomas Fuller (TF) and Rilana Cima (RC) independently screened titles and abstracts from the search results for eligible studies. When there were disagreements at the screening stage, we obtained copies of the full-text articles and examined them closely for eligibility. For all disagreements over full-text articles being assessed for inclusion, a third review author was consulted as an arbiter.

We recorded and presented the flow of study identification and selection in the form of a PRISMA flow chart (Moher 2009; Figure 1). 
Figure 1. Process for sifting search results and selecting studies for inclusion

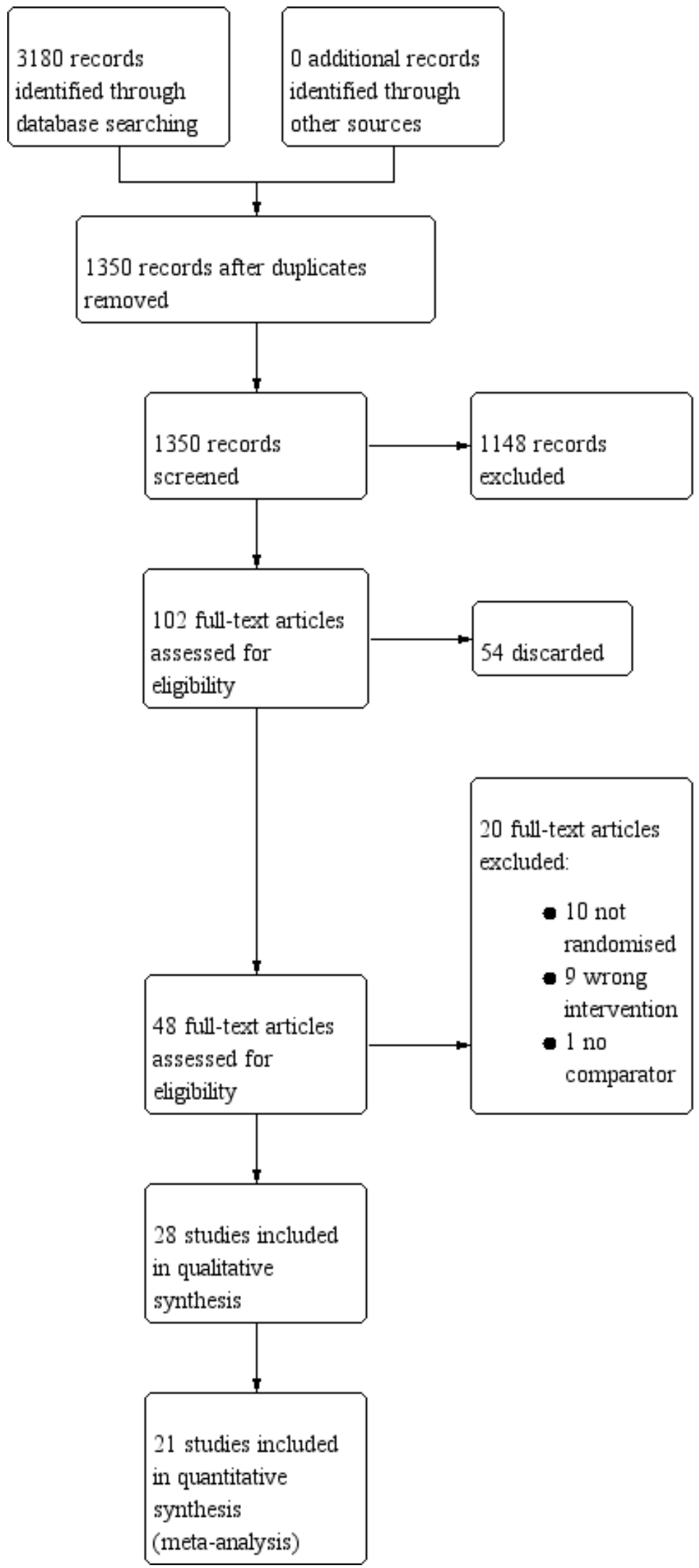




\section{Data extraction and management}

TF co-ordinated the retrieval of full-text articles as well as the management and extraction of all data. Two of TF, Derek Hoare (DH), RC or Birgit Mazurek (BM) independently extracted data from the included studies into standardised data forms based on a generic form developed by the Cochrane ENT editorial group. In the event that one of the review authors was the author of an included study he or she did not extract data from the study. Where relevant, the review authors copied and pasted verbatim text from included studies into the data extraction form. Any disagreements in the data extraction were initially addressed through discussion between the review authors involved. If that did not lead to agreement, a third review author was consulted as an arbiter. In the event of information not being reported in adequate detail to enable decisions about inclusion or exclusion, we contacted (or at least attempted multiple times to do so) study authors to request the provision of additional information.

Data extraction included information on the following: details of the source of participants, eligibility criteria, methods, participants, intervention treatment elements, outcome measures at baseline (or pre-test) and other time points reported in the respective studies, results including estimates of effects and confidence intervals, details of the funding source, key conclusions from the authors, comments from the review authors especially with regard to any differences between protocols and study reports, details of any correspondence required and any references to other relevant studies. Further details of the data to be extracted for intervention reviews are specified in table 7.2 of the Cochrane Handbook for Systematic of Interventions (Higgins 2011).

At the completion of data collection and once there was agreement on the data set that had been extracted, we entered the data into Review Manager 5.3 (RevMan 2014).

\section{Assessment of risk of bias in included studies}

TF, BM, DH and RC completed assessment of the risk of bias of the included studies independently, with the following taken into consideration, as guided by theCochrane Handbook for Systematic Reviews of Interventions (Higgins 2011):

- sequence generation;

- allocation concealment;

- blinding;

- incomplete outcome data;

- selective outcome reporting; and

- other sources of bias.

We used the Cochrane 'Risk of bias' tool in RevMan 5.3 (RevMan 2014), which involved describing each of these domains as reported in the study and then assigning a judgement about the adequacy of each entry: 'low', 'high' or 'unclear' risk of bias. In the event of disagreement between assessors of risk of bias, we discussed the rationale for the respective judgements in an effort to resolve the differences. If that did not lead to agreement, a third review author acted as an arbiter.

\section{Measures of treatment effect}

We analysed ordinal data as if it were continuous data and used standardised mean differences (SMD) and Cohen's d effect size measurement to estimate treatment effects for measures of the impact of tinnitus on quality of life and other continuous measures of secondary outcomes. If feasible, we also pooled data from the same scale and used mean differences (MD).

We analysed dichotomous data using risk ratios (RR) and reported all results with $95 \%$ confidence intervals $(95 \% \mathrm{Cls})$.

\section{Unit of analysis issues}

One study used a cluster-randomised design so we chose statistical methods in consultation with a statistician and following the recommendations in the Cochrane Handbook for Systematic Reviews of Interventions to "extract an estimate of the required effect measure from an analysis that accounts for the cluster design" using an odds ratio with confidence interval or generalised estimating equations (Higgins 2011). Also as specified we used the inverse variance method to meta-analyse effect estimates and standard errors so that the clustered nature of the data was taken into consideration (Higgins 2011).

We did not include any RCTs that used a cross-over design. Had we done so, individual participant data constituting the unit of analysis from the first treatment phase would have been included in the meta-analysis.

\section{Dealing with missing data}

Whenever possible we attempted to contact investigators to request missing data relating to, for example, study characteristics, outcome measures and how many patients dropped out or were included in the analysis. In relation to missing information about dropout or numbers included in the analysis, if we did not receive a response or data from the authors, we conducted the analysis using a conservative approach and assumed that the missing participants' data indicated no effect of/from the intervention. We undertook a sensitivity analysis to examine the effect of this assumption by comparing the results with what would happen if the missing participants had the best possible outcome.

In one study standard deviations were not reported for the Tinnitus Effects Questionnaire (TEQ) total score (Jakes 1992). It was not possible, from the information reported in Jakes 1992, to estimate the standard deviations, so we made a decision to use the standard deviation reported in Henry 1998a.

Where there were missing standard deviations for continuous data, we used methods to estimate these using confidence intervals, standard errors, $\mathrm{t}, \mathrm{P}$ or $\mathrm{F}$ values where reported.

We report the attempts to contact authors for missing data and responses (or otherwise), along with consideration of the potential impact of the missing data, in the Discussion of the review.

\section{Assessment of heterogeneity}

We investigated clinical heterogeneity with regard to: components of the interventions, mode of delivery, level of action, who delivered the CBT and the type of intervention used in the control condition. We also assessed methodological heterogeneity according to study design and risk of bias (i.e. randomisation, blinding of outcome assessment, losses to follow-up).

We assessed the degree of statistical heterogeneity that existed across studies using the $\mathrm{I}^{2}$ statistic and we used the following from 
the Cochrane Handbook for Systematic Reviews of Interventions as a guide for interpretation (Higgins 2011):

- $0 \%$ to $40 \%$ : might not be important;

- $30 \%$ to $60 \%$ : may represent moderate heterogeneity;

- $50 \%$ to $90 \%$ : may represent substantial heterogeneity;

- $75 \%$ to $100 \%$ : considerable heterogeneity.

\section{Assessment of reporting biases}

We examined reporting bias through the creation of funnel plots for the comparisons of CBT versus no intervention/wait list control and $\mathrm{CBT}$ versus any other active comparator.

\section{Data synthesis}

We conducted meta-analyses using random-effects models as we expected that there would be differences between the study populations and methods used. We conducted sensitivity analyses using fixed-effect models.

We pooled studies where there was sufficient similarity between them with regard to: outcome (good convergent validity), level of action (i.e. individual or group therapy) and mode of delivery (i.e. in person, face-to-face or online).

We stratified studies into four comparisons:

- CBT versus no intervention/waiting list control;

- CBT versus usual audiological care (tinnitus education and rehabilitation for hearing loss);

- CBT versus TRT (directive counselling and bilateral masking);

- CBT versus other experimental control (pooled if using the same experimental control). Other experimental controls may include transcranial magnetic stimulation, electrical or electromagnetic stimulation therapy or bio- neuro-feedback.

The intention was to pool the results of the CBT treatments. While CBT treatment protocols differed we judged them, within the particular sub-types of CBT, to be similar enough to conduct metaanalyses although there was significant statistical heterogeneity.

\section{Subgroup analysis and investigation of heterogeneity}

We conducted the following subgroup analyses for the primary outcome of the impact of tinnitus on quality of life:

- Studies by types of therapy: 'cognitive only', 'behavioural only', 'cognitive and behavioural only', ACT, mindfulness.

- Studies by modes of delivery: 'face-to-face' or 'online CBT'.

- Studies by unit of delivery: 'individual patient therapy' or 'group therapy'.

- Study or patient groups by who delivers CBT; 'psychologists' or 'psychiatrists' or 'audiologists' or other therapists or clinicians.

- Studies by whether participants are included/excluded according to their hearing status: 'hearing loss was an exclusion criterion' or 'hearing loss was not an exclusion criterion'.

\section{Sensitivity analysis}

We conducted the following sensitivity analyses to examine the role of:
- meta-analysis using random-effects and fixed-effect models respectively;

- including or excluding studies at high risk of bias for incomplete outcome data.

- replacing missing data with a conservative compared with an 'optimistic' approach in the event that data within a particular study were not collected (or reported) at the end of treatment.

\section{Summary of findings and assessment of the certainty of the evidence}

We used the GRADE approach to rate the overall certainty of evidence. The certainty of evidence reflected the extent to which we were confident that an estimate of effect was correct and we applied this to the interpretation of results. There were four possible ratings: high, moderate, low, and very low. A rating of high certainty of evidence implied that we were confident in our estimate of effect and that further research is very unlikely to change our confidence in the estimate of effect. A rating of very low certainty implied that any estimate of effect obtained is very uncertain.

The GRADE approach rates evidence from RCTs that do not have serious limitations as high certainty. However, several factors can lead to the downgrading of the evidence to moderate, low, or very low. The degree of downgrading is determined by the seriousness of these factors:

- study limitations (risk of bias);

- inconsistency;

- indirectness of evidence;

- imprecision; and

- publication bias.

'Summary of findings' tables for CBT compared with no intervention/waiting list control, usual audiological care, TRT and other control interventions are presented (Summary of findings for the main comparison; Summary of findings 2; Summary of findings 3; Summary of findings 4). The tables include the following outcomes: impact of tinnitus on quality of life, serious adverse effects, depression, anxiety, health-related quality of life, negatively biased interpretations of tinnitus and other adverse effects.

\section{RES U L T S}

\section{Description of studies}

\section{Results of the search}

The Cochrane ENT Information Specialist conducted an electronic search of the literature in November 2019. A total of 3180 records were identified through this method, of which 1350 remained after duplicates were removed. We excluded 1148 references on the basis of title or abstract and retrieved a total of 102 records for fulltext review. We discarded 54 records and excluded 20 (10 because the allocation of participants was not randomised, nine because the interventions were not CBT and one because there was not a relevant comparator). See Characteristics of excluded studies for details. Five records were for ongoing studies (see Characteristics of ongoing studies). There are no studies awaiting assessment.

In total we included 28 studies in this review. Twenty-two of these studies reported quantitative data, which were included 
in meta-analyses (Abbott 2009; Andersson 2002; Andersson 2005; Arif 2017; Beukes 2018a; Beukes 2018b; Cima 2012; Davies 1995; Henry 1996; Hesser 2012; Jakes 1992; Jasper 2014; Kaldo 2007; Kreuzer 2012; Malinvaud 2016; McKenna 2017; Nyenhuis 2013a; Oron (unpublished); Philippot 2012a; Schmidt 2018; Weise 2016; Westin 2011). Six studies did not present usable data (Henry 1998; Jakes 1986; Lindberg 1989; Martz 2018; Robinson 2008; Zhong 2014).

No additional studies were identified through other search methods, which included contacting researchers and handsearching the references of included studies.

Figure 1 presents the study retrieval and selection process, and reasons for exclusion.

\section{Included studies}

We included 27 published studies and one unpublished study that is being prepared for publication (Oron (unpublished)). For descriptions of the studies, see the Characteristics of included studies table.

\section{Design}

Twenty-seven studies were parallel-group RCTs and one was a cluster-RCT (Abbott 2009).

Three studies had multiple intervention/treatment arms (Jasper 2014; Hesser 2012; Martz 2018). Jasper 2014 was a three-arm trial in which Internet-based CBT (iCBT) and group-CBT was compared with an Internet-based discussion forum. Hesser 2012 was also a three-arm trial but compared a CBT intervention with an ACT intervention and an online discussion forum condition respectively. Martz 2018 examined the efficacy of CBT, ACT, Coping Effectiveness Training and a wait list control condition.

\section{Sample sizes}

The total sample size for all included studies was 2733 . Within studies the sample size ranged from 23 (Andersson 2005) to 492 (Cima 2012) participants.

\section{Setting}

Nine studies were set in hospitals. Two of these were in England (Davies 1995; McKenna 2017), two in Sweden (Lindberg 1989; Westin 2011), and one each in China (Zhong 2014), France (Malinvaud 2016), Israel (Oron (unpublished)), the Netherlands (Cima 2012), and Wales (Arif 2017). A total of five studies, in four countries, were conducted online (i.e. using Internet-based interventions): two in Sweden (Andersson 2002; Hesser 2012), and one in Australia (Abbott 2009), England (Beukes 2018a), and Germany (Weise 2016) respectively. In one study the intervention (iCBT) was conducted online while the comparator, audiological treatment as usual was conducted in hospital settings (Beukes 2018b).

Three studies were conducted in Veterans Affairs clinics in the USA (Martz 2018; Robinson 2008; Schmidt 2018) and two studies were conducted in psychology clinics (one in Belgium (Philippot 2012a) and one in Sweden (Andersson 2005)). Nyenhuis 2013a conducted a study with four arms that included an online condition, a bibliotherapy condition and interventions delivered face-to-face in two "study centres" in the southern region of
Lower Saxony, Germany. One study, set in Sweden, delivered the intervention primarily through bibliotherapy (Kaldo 2007). Two of three conditions in Jasper 2014 (set in Germany) were delivered as an Internet-based intervention (i.e. iCBT and the control condition), while the setting for group CBT was not described. Five studies did not report the setting in which the studies were conducted; two were from Australia (Henry 1996; Henry 1998), two were from England (Jakes 1986; Jakes 1992), and one was from Germany (Kreuzer 2012).

Of the 28 included studies, six were from England, six from Sweden, four from Germany, three from the USA, three from Australia, and one each from Belgium, China, France, Israel, the Netherlands and Wales.

\section{Participants}

All studies included adult participants (18 years or over) with the mean age of participants ranging from 42.6 years to 70.1 years. Six studies limited the maximum age of participants: one limited it to 65 years (Abbott 2009); three to 70 years (Andersson 2002; Jakes 1986; Malinvaud 2016); one to 75 years (Nyenhuis 2013a); and one to 80 years (Kreuzer 2012). One study had a minimum age of 65 years (Andersson 2005). Eleven studies did not report inclusion or exclusion criteria related to age (Davies 1995; Henry 1996; Henry 1998; Jakes 1992; Jasper 2014; Lindberg 1989; Martz 2018; Oron (unpublished); Philippot 2012a; Robinson 2008; Zhong 2014).

Of all the participants in the included studies, $40.7 \%$ were female $(n=1106)$ and $58.1 \%$ were male $(n=1579)$. There were missing data on gender for 34 participants from five studies (Abbott 2009; Davies 1995; Henry 1996; Malinvaud 2016; Schmidt 2018), although one study accounted for approximately $44 \%$ of this $(n=15)$ (Davies 1995). Three of the 28 studies had a greater proportion of female than male participants (53.5\%, 52.4\% and $59.7 \%$ respectively) (Arif 2017; Jakes 1992; Weise 2016). The proportion of males in the included studies ranged from 28.9\% (Davies 1995) to 82.1\% (Abbott 2009).

The reported tinnitus duration ranged from a minimum average of 3.2 months (Nyenhuis 2013a) to a maximum average of 22.9 years (Schmidt 2018). A minimum tinnitus duration was not required/ reported in nine studies (Arif 2017; Beukes 2018b; Cima 2012; Jakes 1986; Martz 2018; Oron (unpublished); Philippot 2012a; Robinson 2008; Zhong 2014), although three did require a referral and/or diagnosis from a medical professional such as an Ear, Nose and Throat surgeon or general practitioner (Beukes 2018b; Cima 2012; Philippot 2012a). Seven studies specified that participants had a diagnosis from a medical professional as part of their inclusion criteria (Abbott 2009; Andersson 2002; Henry 1996; Henry 1998; Hesser 2012; Kaldo 2007; Weise 2016).

Most studies (24 of the 28 ) stated or described in their inclusion criteria a level of tinnitus severity required to participate. Ten studies gave cut-off scores on self-report questionnaires as criteria indicating minimum levels of severity. Within this group, there was some variation on the specific cut-off scores and questionnaires referred to. Specifically, Jasper 2014 required participants to have a minimum score of 18 on the THI, compared with others who required minimums of 20 (Schmidt 2018), 30 (Westin 2011) or 38 (Hesser 2012; Weise 2016). Jasper 2014 and Weise 2016 also specified additional cut-off scores on the mini-TQ ( 8 and 13 respectively) and Schmidt 2018 specified a minimum score of 17 on 
the TRQ, and 5 or more on the Tinnitus Impact Screening Interview. Three studies referred solely to a TRQ score, though there was also a difference in cut-offs (Henry 1996; Henry 1998; Kaldo 2007); 10 or more for Kaldo 2007, and 17 or more for Henry 1996 and Henry 1998. Martz 2018 required prospective participants to have a minimum score of 21 and Beukes 2018a required participants to have a minimum of 25 on the Tinnitus Functional Index (TFI). Descriptions and/or indicators of tinnitus severity referred to in other studies included for example: "primary complaint of tinnitus" (e.g. Cima 2012), "self-reported distress due to tinnitus" (Robinson 2008), and "significant psychological distress and impairment in everyday activities resulting from tinnitus" (Philippot 2012a).

One study excluded participants with severe hearing loss due to the impact this could have on the use of wearable sound generators (Westin 2011), but otherwise hearing loss was not applied as an exclusion criterion for participating in the studies.

In relation to co-morbid psychological conditions, 16 studies included measures of anxiety and 23 included measures of depression. However, only three studies specifically referred to anxiety or depression in their inclusion or exclusion criteria (Andersson 2005; Kaldo 2007; Weise 2016). Kaldo 2007 specified that participants must have scores lower than 19 on both the anxiety and depression subscales of the Hospital Anxiety and Depression Scale. Andersson 2005 included people with scores lower than 22 on the Beck Depression Inventory, and Weise 2016 included those without a "clinical diagnosis of depression". It was, however, more common (15 out of 28 studies) that descriptive criteria about psychopathology were used to exclude potential participants. For example, criteria would refer to prospective participants with/without the presence/absence of a major psychiatric condition or disorder (Beukes 2018a; Davies 1995; Jakes 1992). Five studies also specified high risk of suicide in their exclusion criteria (Andersson 2005; Hesser 2012; Jasper 2014; McKenna 2017; Weise 2016). Other specific psychological conditions referred to in participant selection criteria included substance use disorders (McKenna 2017; Schmidt 2018; Weise 2016), psychosis (Robinson 2008; Schmidt 2018), and personality disorders (Philippot 2012a).

\section{Interventions and comparisons}

Cognitive, behavioural, ACT, mindfulness, and cognitive and behavioural (combined) interventions were considered as 'CBT' and thus eligible for inclusion in this review. (Note that in the following description of the studies, some had more than one CBT and/or control arm within the study, and thus the total number of comparisons does not equal 28). Seventeen studies tested CBT (Abbott 2009; Andersson 2002; Andersson 2005; Beukes 2018a; Beukes 2018b; Cima 2012; Jasper 2014; Kaldo 2007; Lindberg 1989; Malinvaud 2016; Martz 2018; Nyenhuis 2013a; Robinson 2008; Schmidt 2018; Zhong 2014); five tested cognitive interventions (Davies 1995; Henry 1996; Henry 1998; Jakes 1986; Jakes 1992), four tested ACT (Hesser 2012; Martz 2018; Oron (unpublished); Westin 2011), and four tested mindfulness interventions. Within the mindfulness interventions, two tested mindfulness meditation (Arif 2017; Kreuzer 2012), one tested a mindfulness-based stress reduction (Philippot 2012a), and one tested a mindfulness-based cognitive therapy intervention (McKenna 2017). No studies tested purely behavioural interventions.
The most common mode by which interventions were delivered was face-to-face. Twenty-one studies delivered CBT face-to-face (Andersson 2005; Arif 2017; Cima 2012; Davies 1995; Henry 1996; Henry 1998; Jakes 1986; Jakes 1992; Jasper 2014; Kreuzer 2012; Lindberg 1989; Malinvaud 2016; Martz 2018; McKenna 2017; Nyenhuis 2013a; Oron (unpublished); Philippot 2012a; Robinson 2008; Schmidt 2018; Westin 2011; Zhong 2014), six delivered CBT in the form of an Internet-based intervention (Abbott 2009; Andersson 2002; Beukes 2018a; Beukes 2018b; Hesser 2012; Weise 2016), and one multi-arm study included an Internet-based and faceto-face CBT condition (Jasper 2014). Kaldo 2007 compared CBT delivered as bibliotherapy with email contact with a wait list control condition. Seventeen studies delivered CBT in a group format (Andersson 2005; Cima 2012; Henry 1996; Henry 1998; Jakes 1986; Jakes 1992; Jasper 2014; Kreuzer 2012; Lindberg 1989; Malinvaud 2016; Martz 2018; McKenna 2017; Nyenhuis 2013a; Oron (unpublished); Philippot 2012a; Robinson 2008; Schmidt 2018; Zhong 2014), 10 studies delivered CBT individually (Abbott 2009; Andersson 2002; Arif 2017; Beukes 2018a; Beukes 2018b; Davies 1995; Jakes 1986; Kaldo 2007; Weise 2016; Westin 2011), and one study included an individual and group CBT condition (Jasper 2014).

Professions involved in delivering interventions included psychologists (Abbott 2009; Andersson 2005; Davies 1995; Henry 1996; Hesser 2012; Jasper 2014; Kaldo 2007; Lindberg 1989; Malinvaud 2016; Martz 2018; McKenna 2017; Nyenhuis 2013a; Philippot 2012a; Robinson 2008; Schmidt 2018; Weise 2016; Westin 2011), and audiologists (Beukes 2018a; Beukes 2018b). In one study a multidisciplinary team delivered CBT (Cima 2012), and in another psychologists and psychiatrists delivered the intervention (Robinson 2008). Three studies described the people delivering the interventions as "therapists" without providing details of qualifications (Arif 2017; Jakes 1992; Kreuzer 2012), and four studies did not report any information about who delivered the intervention (Henry 1998; Jakes 1986; Oron (unpublished); Zhong 2014).

\section{CBT versus no intervention/waiting list control}

Fourteen studies compared CBT to wait list control conditions (Andersson 2002; Andersson 2005; Beukes 2018a; Henry 1996; Henry 1998; Jakes 1992; Kaldo 2007; Kreuzer 2012; Lindberg 1989; Malinvaud 2016; Martz 2018; Oron (unpublished); Robinson 2008; Westin 2011). The duration of the waiting list control period ranged from 3 (Lindberg 1989) to 22 (Kreuzer 2012) weeks, with the median being 6 weeks, and the average waiting period being 8.1 weeks. In all studies, participants were offered the CBT intervention at the end of the waiting period.

\section{CBT versus usual audiological care (tinnitus education and} rehabilitation for hearing loss)

Three studies compared CBT to audiological care (Beukes 2018b; Cima 2012; Schmidt 2018). Beukes 2018b compared an individually delivered, eight-week iCBT (with optional email contact with an audiologist) intervention to audiological care as usually delivered in the UK; that is, three 60-minute appointments, and two followup appointments at one and two months respectively. In Cima 2012, the CBT intervention was delivered face-to-face, according to a stepped-care model where those requiring greater assistance received a greater number of sessions. The audiological care condition in Cima 2012 was based on the results from a survey of audiologists asking what care they provided to patients with 
tinnitus, as at the time there was no standardised audiological care for tinnitus in the Netherlands. The audiological care in Cima 2012 also comprised a stepped-care approach where patients first had audiological tests and education in step 1, and then if needed in step 2, up to nine sessions with a social worker. Schmidt 2018 tested a six-week face-to-face group CBT intervention developed specifically for veterans of military service. Audiological care was also delivered in groups over six weeks, and included tinnitus education and attentional skills training (Schmidt 2018).

\section{CBT versus tinnitus retraining therapy}

One study compared CBT to TRT and a wait list control condition (Westin 2011). The CBT intervention comprised Acceptance and Commitment Therapy (ACT) delivered individually over the course of 10 weeks in 60- to 75-minute sessions. TRT involved a 2.5-hour consultation with an ENT physician which included a diagnostic assessment, and directive counselling. Participants in the TRT condition were also fitted with bilateral sound generators (as per TRT protocol) and instructed to use them for a minimum of eight hours per day over an 18-month period.

\section{CBT versus other active control}

Sixteen studies compared CBT to an active experimental control group not otherwise included in the previous comparisons (Abbott 2009; Arif 2017; Davies 1995; Henry 1996; Hesser 2012; Jakes 1986; Jakes 1992; Jasper 2014; Malinvaud 2016; Martz 2018; McKenna 2017; Nyenhuis 2013a; Oron (unpublished); Philippot 2012a; Weise 2016; Zhong 2014).

The CBT interventions included: CBT (Abbott 2009; Jakes 1986; Jasper 2014; Malinvaud 2016; Martz 2018; Nyenhuis 2013a; Weise 2016; Zhong 2014), cognitive therapy (Davies 1995; Henry 1998; Jakes 1992), ACT (Hesser 2012; Martz 2018; Oron (unpublished), and mindfulness (Arif 2017; McKenna 2017; Philippot 2012a). Eleven CBT interventions were delivered face-to-face (Arif 2017; Davies 1995; Henry 1996; Jakes 1986; Jakes 1992; Malinvaud 2016; Martz 2018; McKenna 2017;Nyenhuis 2013a; Oron (unpublished); Zhong 2014), and three were delivered as Internet-based interventions (Abbott 2009; Hesser 2012; Weise 2016). Jasper 2014 included a group CBT and iCBT arm in addition to a control condition.

CBT was provided individually in five studies (Abbott 2009; Arif 2017; Davies 1995; Hesser 2012; Weise 2016), in groups in nine studies (Henry 1996; Jakes 1986; Jakes 1992; Malinvaud 2016; Martz 2018; McKenna 2017; Nyenhuis 2013a; Oron (unpublished); Philippot 2012a), and both individually and in groups in Jasper 2014. In one study, no information was reported to indicate whether participants engaged in treatment individually or in groups (Zhong 2014).

In nine studies psychologists delivered CBT (Davies 1995; Henry 1996; Jakes 1986; Jasper 2014; Malinvaud 2016; Martz 2018; McKenna 2017; Nyenhuis 2013a; Philippot 2012a), four studies tested guided Internet-based interventions by psychologists who were available via email to answer questions and provide feedback (Abbott 2009; Hesser 2012; Jasper 2014; Weise 2016), and two studies reported that "therapists" delivered the intervention but did not provide further information on their qualifications or experience (Arif 2017; Jakes 1992). One study, Zhong 2014, did not report what professional delivered the CBT.

Other experimental control interventions included:
- Relaxation (Arif 2017; Davies 1995; Jakes 1986; McKenna 2017; Philippot 2012a). The types of relaxation used as active control conditions included: applied relaxation based on the work of Bernstein 1973 (Jakes 1986), Bernstein 1984 (Davies 1995), Jacobson 1957 (Philippot 2012a), and Ost 1987 (Arif 2017; McKenna 2017), respectively. In each of these studies, the same people who delivered the CBT delivered the relaxation therapy.

- Provision of information about tinnitus and hearing (Abbott 2009; Henry 1996; Nyenhuis 2013a). This included information about tinnitus and its causes, the auditory system, audiological assessment and available treatments. Information about tinnitus was provided by: a clinical psychologist (Henry 1996), an 11-page booklet (Nyenhuis 2013a), and computer/Internet with the support of brief contact from a psychologist to provide new passwords to access new information support regarding their tinnitus coping.

- Internet-based discussion forums (Hesser 2012; Jasper 2014; Weise 2016). The discussion forums were moderated by clinical psychology students (Hesser 2012; Jasper 2014; Weise 2016), licensed CBT therapists (Weise 2016) or licensed psychologists (Hesser 2012). New topics of discussion, such as "representations of tinnitus in the media" (Weise 2016) were presented on a weekly basis in addition to participants being able to initiate topics in each of the respective studies.

- Coping effectiveness training (Martz 2018; Oron (unpublished)) aimed to increase understanding of stress and coping with tinnitus, and how to better learn how to match appropriate coping strategies to situations. The intervention content was delivered in English by psychologists or counsellors (Martz 2018), and in Hebrew (Oron (unpublished)) - no information available about the presenters).

- Masking (Jakes 1992; Zhong 2014). Jakes 1992 used a standard masker device supplied by $\mathrm{A}$ and $\mathrm{M}$ Hearing Aids Ltd and instructed participants to turn the masker volume up so that they could not hear the tinnitus. Jakes 1992 also included a condition with a placebo masking device that was the same as in the masking condition, but the volume control of the masker was glued into place at the participant's threshold. Zhong 2014 used an MP3 player (no further details provided) as a masking device and participants were instructed to use it one to three times a day, for 15 to 20 minutes each time.

- Virtual reality was delivered in two steps; the first of which included information about tinnitus, treatment and short breathing and relaxation techniques (Malinvaud 2016). The second step where participants entered into a virtual world and were able to control a tinnitus avatar took place under the direction of an ENT physician over eight once-weekly sessions. During a session (one-hour duration) participants were asked to navigate through three environments in which they could choose to displace, mask or unmask sounds as they wished (Malinvaud 2016).

- Self-help.Nyenhuis 2013a included two self-help conditions which varied only in the mode of delivery, i.e. via bibliotherapy or Internet. The content of these conditions was adapted from Tinnitus Coping Training (Kröner-Herwig 1997; Kröner-Herwig 2003). (See also Characteristics of included studies).

Five studies also included a wait list control condition in addition to an active control group (Henry 1996; Jakes 1992; Malinvaud 2016; Martz 2018; Oron (unpublished). 


\section{Outcomes}

\section{Primary outcomes}

\section{Impact of tinnitus on quality of life}

Twenty-six of the 28 studies reported changes in the impact of tinnitus on quality of life as measured by scores on a multiitem questionnaire. Fourteen studies used a single multi-item questionnaire (Abbott 2009; Andersson 2002; Andersson 2005; Arif 2017; Beukes 2018a; Davies 1995; Hesser 2012; Jakes 1986; Jakes 1992; Nyenhuis 2013a; Oron (unpublished); Philippot 2012a; Westin 2011; Zhong 2014), nine studies used two multi-item questionnaires (Beukes 2018b; Cima 2012; Jasper 2014; Kaldo 2007; Kreuzer 2012; Malinvaud 2016; McKenna 2017; Schmidt 2018; Weise 2016), two studies used three multi-item questionnaires (Henry 1996; Henry 1998), and one study used four multi-item questionnaires (Robinson 2008).

Of the 14 studies that used one multi-item questionnaire, four used the Tinnitus Handicap Inventory (THI; Newman 1996) (Hesser 2012; Oron (unpublished); Westin 2011; Zhong 2014), four used the Tinnitus Reaction Questionnaire (TRQ; Wilson 1991) (Abbott 2009; Andersson 2002; Andersson 2005; Arif 2017), three used the Tinnitus Effects Questionnaire-Emotional Distress scale (TEQ-ED; Hallam 1988) (Davies 1995; Jakes 1986; Jakes 1992), one used the Tinnitus Functional Index (TFI; Meikle 2012) (Beukes 2018a), one used the Tinnitus Questionnaire (TQ; Hallam 1988; Hallam 2008) (Nyenhuis 2013a), and one used the Tinnitus Psychological Impact Questionnaire (QIPA) (Philippot 2012a). (See explanatory note in Characteristics of included studies, Philippot 2012a regarding the QIPA).

Of the nine studies that used two questionnaires, Beukes 2018b used the TFI and THI; Jasper 2014 and Weise 2016 both used the THI and mini-TQ, Malinvaud 2016 used the THI and THQ; Schmidt 2018 and Kaldo 2007 used the THI and TRQ; Kreuzer 2012 and Cima 2012 used the THI and TQ; and, McKenna 2017 used the TFI and TQ. Henry 1996 and Henry 1998 both used the same three questionnaires (TRQ, THQ, TEQ-ED) and Robinson 2008 used four multi-item questionnaires (THQ, TRQ, THI, TQ)

In this review we measured outcome at treatment end, and at six- and 12-month follow-up. Three studies reported follow-up data at six months (Jasper 2014; McKenna 2017; Weise 2016), and six studies reported data at 12 months follow-up (Andersson 2002; Henry 1996; Hesser 2012; Kaldo 2007; Robinson 2008; Weise 2016). However, only McKenna 2017 and Henry 1996 reported data from active comparator groups that could be considered. Other studies used wait list control groups and subsequently gave those participants CBT. The time points at which follow-up data were collected in other studies included two weeks (Kreuzer 2012), one month (Davies 1995; Jakes 1986; Martz 2018; McKenna 2017; Zhong 2014), two months (Beukes 2018a; Beukes 2018b; Robinson 2008; Schmidt 2018), three months (Andersson 2005; Jakes 1992; Malinvaud 2016; Philippot 2012a), four months (Cima 2012; Davies 1995; Henry 1996; Jakes 1986; Robinson 2008; Zhong 2014), 4.5 months (Westin 2011), eight months (Henry 1996), nine months (Nyenhuis 2013a), and 16 months (Westin 2011). Four studies did not collect follow-up data (Abbott 2009; Arif 2017; Lindberg 1989; Oron (unpublished)).

\section{Serious adverse effects}

Eleven of the 28 studies provided information about the incidence of serious adverse effects. Seven studies reported, or we were informed by the authors via personal communication, that there were no serious adverse effects associated with CBT (Andersson 2002; Andersson 2005; Beukes 2018b; Cima 2012; Kaldo 2007; Malinvaud 2016; Oron (unpublished)). Four studies reported some serious adverse effects (mostly symptom deterioration) in a small number of participants (Beukes 2018a; Nyenhuis 2013a; Weise 2016; Westin 2011). Information about the presence of serious adverse effects was available at the end of treatment (Beukes 2018a; Nyenhuis 2013a; Westin 2011), six-month follow-up (Westin 2011) and 12-month follow-up (Beukes 2018a as reported in Beukes 2018c).

\section{Secondary outcomes}

\section{Depression}

Depression was measured with multi-item questionnaires in 22 studies. It was measured with the Hospital Anxiety and Depression Scale-Depression subscale (HADS-D; Zigmond 1983) in 11 studies (Andersson 2002; Andersson 2005; Arif 2017; Cima 2012; Hesser 2012; Jasper 2014; Kaldo 2007; Malinvaud 2016; McKenna 2017; Weise 2016; Westin 2011), the Beck Depression Inventory (BDI; Beck 1996) in five studies (Davies 1995; Henry 1996; Henry 1998; Kreuzer 2012; Philippot 2012a), the Patient Health Questionnaire-9 (PHQ-9; Kroenke 2001) in three studies (Beukes 2018a; Beukes 2018b; Nyenhuis 2013a), the Depression Anxiety and Stress ScaleDepression subscale (DASS-D; Lovibond 1995) in one study (Abbott 2009), and the Hamilton Rating Scale for Depression (HRSD; Hamilton 1960) in one study (Jakes 1986). One study used both the HRSD and the BDI to measure depression (Robinson 2008).

\section{Anxiety}

Anxiety was measured with multi-item questionnaires in 16 studies. It was measured with the Hospital Anxiety and Depression ScaleAnxiety subscale (HADS-A; Zigmond 1983) in nine studies (Arif 2017; Cima 2012; Hesser 2012; Jasper 2014; Kaldo 2007; Malinvaud 2016; McKenna 2017; Weise 2016; Westin 2011); the Generalised Anxiety Disorder-7 scale (GAD-7; Spitzer 2006) in two studies (Beukes 2018a; Beukes 2018b); the State Trait Anxiety Inventory (STAI; Spielberger 1983) in two studies (Davies 1995; Philippot 2012a); and the Depression Anxiety and Stress Scale-Anxiety subscale (DASS-A; Lovibond 1995) in one study (Abbott 2009). Two studies used both the HADS-A and Anxiety Sensitivity Index (ASI; Reiss 1986) to assess anxiety (Andersson 2002; Andersson 2005).

\section{Quality of life}

Seven studies assessed change in quality of life. Quality of life was assessed with multi-item questionnaires that included the Satisfaction with Life Survey (SWLS; Diener 1985) in two studies (Beukes 2018a; Beukes 2018b); the Quality of Life Inventory (QOLI; Frisch 1992) in two studies (Hesser 2012; Westin 2011); the Health Utilities Index (HUI; Furlong 2001) in one study (Cima 2012); the Quality of Well-being Scale (Kaplan 1996) in one study (Robinson 2008); and the WHOQoL (Skevington 2004) in one study (Abbott 2009).

\section{Negative biased interpretations of tinnitus}

Biased interpretations of tinnitus were measured with multiitem questionnaires in 10 studies. The Tinnitus Acceptance 
Questionnaire (TAQ; Weise 2013) was used in five studies (Hesser 2012; Jasper 2014; McKenna 2017; Weise 2016; Westin 2011); the Tinnitus Effects Questionnaire-Irrational Beliefs subscale (TEQ-IB; Hallam 1988) in four studies (Davies 1995; Jakes 1992; Henry 1996; Henry 1998); and the Tinnitus Cognitions Questionnaire (TCQ; Wilson 1998) in two studies (Henry 1996; Henry 1998). Cima 2012 used the Tinnitus Catastrophizing Scale (TCS; Cima 2011b) and Fear of Tinnitus Questionnaire (FTQ; Cima 2011b), and McKenna 2017 used the TAQ (Weise 2013), TCS (Cima 2011b), and Tinnitus-Fear and Avoidance Scale (T-FAS; Kleinstauber 2013).

\section{Other adverse effects}

Only one study reported some 'other' adverse effects (Weise 2016). These adverse effects occurred at 12 months follow-up and referred to some "slight deterioration in sleep quality". (Note: there were no long-term comparator data available as the online discussion comparator group had received CBT by that time).

\section{Non-relevant outcomes}

Two studies did not use any outcomes that were relevant to this review (Lindberg 1989; Martz 2018). Lindberg 1989 reported results from visual analogue scales that were used on a daily basis for one week to measure subjective loudness, discomfort from tinnitus and ability to control the discomfort from tinnitus. Martz 2018 reported results from the Brief COPE scale (Carver 1997), a 28-item version of the COPE inventory (Carver 1989) measuring what techniques, strategies or supports people use to manage challenging situations or experiences. Martz 2018 specifically reported results for three subscales: "engagement coping", which includes, for example, positive re-framing, self-distraction and use of humour, "disengagement coping", which includes, denial, behavioural disengagement and self-blame, and "social support coping", which includes instrumental support, emotional support, venting and religion.

\section{Excluded studies}

From the full-text screening we excluded 15 studies. The main reasons for excluding studies were: non-random allocation of participants (six studies); wrong intervention (six studies); secondary analyses of data (two studies); and no appropriate comparator included (one study). A list of studies and the specific reason for exclusion is in the Characteristics of excluded studies table.

\section{Ongoing studies}

We identified five ongoing studies in our search results (NCT03022084; NCT03386123; NCT04004260; NTR6415; SLCTR/2018/005), which are reported in the Characteristics of ongoing studies table.

\section{Risk of bias in included studies}

A graph showing a summary of the 'Risk of bias' assessments is shown in Figure 2 and the review authors' judgements for each included study are shown in Figure 3.

Figure 2. 'Risk of bias' graph: review authors' judgements about each risk of bias item presented as percentages across all included studies.

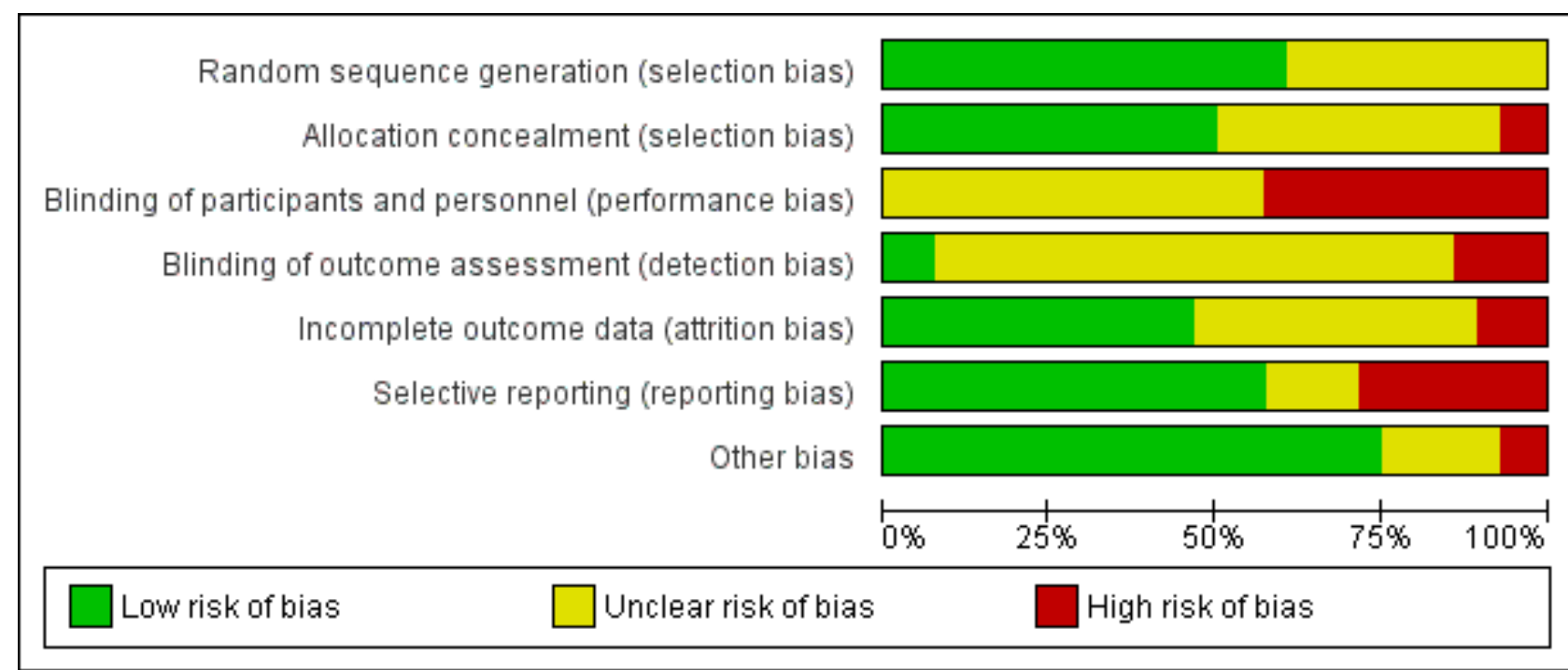


Figure 3. 'Risk of bias' summary: review authors' judgements about each risk of bias item for each included study.

\begin{tabular}{|c|c|c|c|c|c|c|c|}
\hline & 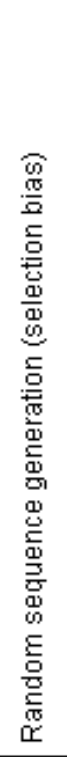 & 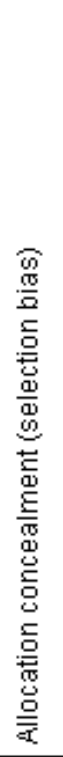 & 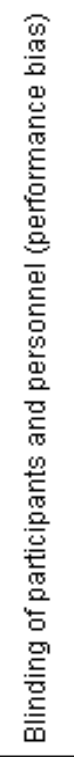 & 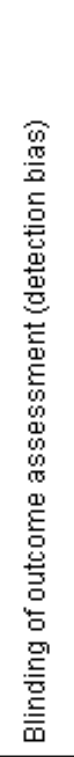 & 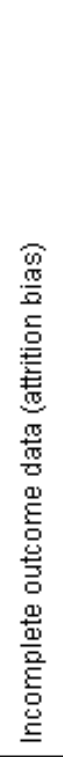 & 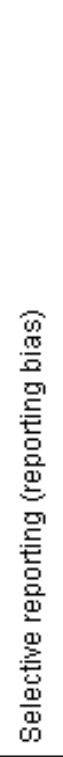 & 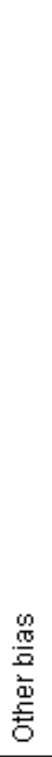 \\
\hline Abbott 2009 & $\odot$ & $?$ & $\odot$ & $?$ & $\odot$ & $\odot$ & $\odot$ \\
\hline Andersson 2002 & $?$ & $\odot$ & 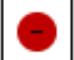 & $?$ & $?$ & + & + \\
\hline Andersson 2005 & $?$ & $?$ & $?$ & $?$ & + & + & + \\
\hline Arif 2017 & $?$ & + & $\odot$ & $?$ & $?$ & + & $\odot$ \\
\hline Beukes 2018a & + & $\odot$ & $\odot$ & $\theta$ & + & + & + \\
\hline Beukes $2018 \mathrm{~b}$ & $\odot$ & $\odot$ & 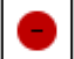 & + & + & + & $\odot$ \\
\hline Cima 2012 & $\odot$ & $\odot$ & $?$ & $?$ & + & + & + \\
\hline Davies 1995 & $?$ & $?$ & $?$ & $?$ & $\odot$ & + & + \\
\hline Henry 1996 & ? & $?$ & $?$ & ? & $\odot$ & $\odot$ & $\odot$ \\
\hline Henry 1998 & $?$ & $?$ & $?$ & $?$ & ? & + & $\odot$ \\
\hline Hesser 2012 & $\odot$ & $\odot$ & $\odot$ & $?$ & + & $\odot$ & + \\
\hline Jakes 1986 & $?$ & $?$ & $?$ & $?$ & $?$ & $\odot$ & + \\
\hline Jakes 1992 & $?$ & $?$ & $?$ & $?$ & $?$ & $\odot$ & $\odot$ \\
\hline Jasper 2014 & + & $\odot$ & $?$ & $\odot$ & + & $?$ & $?$ \\
\hline Kaldo 2007 & + & $?$ & $\odot$ & $?$ & + & + & + \\
\hline Kreuzer 2012 & $\odot$ & $?$ & $\odot$ & $\theta$ & $?$ & $\odot$ & $?$ \\
\hline Lindberg 1989 & $?$ & $\odot$ & $?$ & $?$ & + & $?$ & $?$ \\
\hline Malinvaud 2016 & + & $\odot$ & $\odot$ & $\odot$ & $\odot$ & $\odot$ & $?$ \\
\hline Mart 2018 & $\odot$ & $\odot$ & $\odot$ & $?$ & $?$ & $\odot$ & - \\
\hline McKenna 2017 & + & + & $?$ & $?$ & $?$ & + & $\odot$ \\
\hline
\end{tabular}


Figure 3. (Continued)

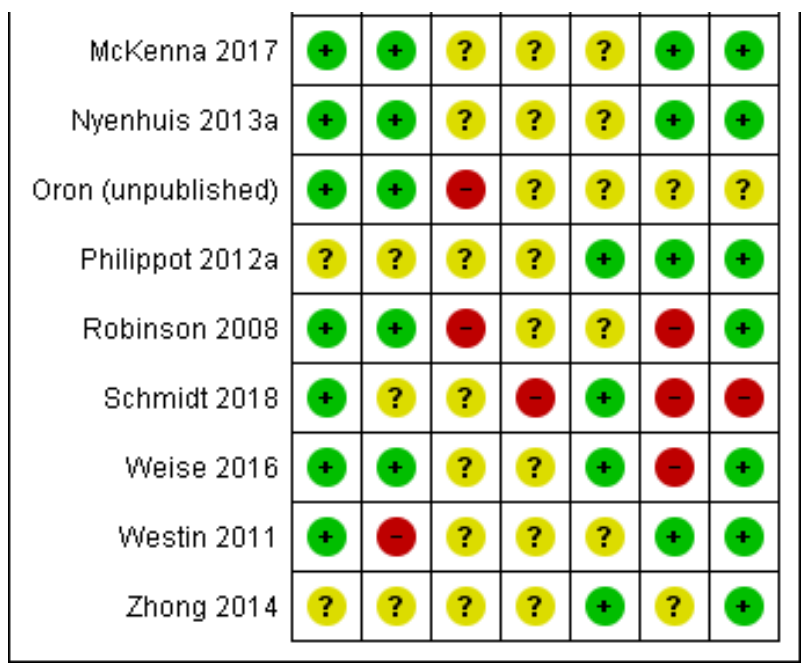

\section{Allocation}

\section{Random sequence generation}

We rated 17 included studies as having low risk of bias because they clearly described or information was received from corresponding authors to confirm an adequate random sequence generation process had been used (Abbott 2009; Beukes 2018a; Beukes 2018b; Cima 2012; Hesser 2012; Jasper 2014; Kaldo 2007; Kreuzer 2012; Malinvaud 2016; Martz 2018; McKenna 2017; Nyenhuis 2013a; Oron (unpublished); Robinson 2008; Schmidt 2018; Weise 2016; Westin 2011). Methods of random sequence generation included Internet-based randomisation systems (e.g. www.random.org, www.randomization.com), coin tossing, computer-generated random number sequences and block randomisation, and drawing numbers from a hat. We rated the remaining 11 studies as having an 'unclear' risk of bias as they did not describe their random sequence generation methods (Andersson 2002; Andersson 2005; Arif 2017; Davies 1995; Henry 1996; Henry 1998; Jakes 1986; Jakes 1992; Lindberg 1989; Philippot 2012a; Zhong 2014).

\section{Allocation concealment}

We rated half the included studies as having a low risk of bias for allocation concealment as it was performed by staff independent of the research team, or was conducted centrally (Andersson 2002; Arif 2017; Beukes 2018b; Cima 2012; Hesser 2012; Jasper 2014; Lindberg 1989; Malinvaud 2016; Martz 2018; McKenna 2017; Nyenhuis 2013a; Oron (unpublished); Robinson 2008; Weise 2016). We rated two studies as being at high risk of bias because there was no allocation concealment (Beukes 2018a; Westin 2011). In the remaining 12 studies, allocation concealment was not described and so we rated those studies as having unclear risk of bias (Abbott 2009; Andersson 2005; Davies 1995; Henry 1996; Henry 1998; Jakes 1986; Jakes 1992; Kaldo 2007; Kreuzer 2012; Philippot 2012a; Schmidt 2018; Zhong 2014).

\section{Blinding}

\section{Blinding of participants and personnel}

We judged 12 studies to be at high risk of bias (Abbott 2009; Andersson 2002; Arif 2017; Beukes 2018a; Beukes 2018b; Hesser
2012; Kaldo 2007; Kreuzer 2012; Malinvaud 2016; Martz 2018; Oron (unpublished); Robinson 2008), and the remaining 16 to be at unclear risk of bias in relation to blinding of participants and personnel (Andersson 2005; Cima 2012; Davies 1995; Henry 1996; Henry 1998; Jakes 1986; Jakes 1992; Jasper 2014; Lindberg 1989; McKenna 2017; Nyenhuis 2013a; Philippot 2012a; Schmidt 2018; Weise 2016; Westin 2011; Zhong 2014). Although CBT itself is not possible to 'mask', we judged there to be a high risk where there was a clear difference between the intervention and comparison group. Specifically, we judged there to be a high risk when there was a wait list control (e.g. Andersson 2002), when an information only control was used (e.g. Abbott 2009), and when participants were explicitly informed of the differences between groups/interventions that they could be allocated to (e.g. Arif 2017). In other scenarios, for example, where participants might not know the difference between the intervention and control, and/or if the content of the alterative treatment was masked, we rated the risk of bias as unclear (e.g. Cima 2012).

\section{Blinding of outcome assessment}

We rated two studies as having a low risk of bias (Beukes 2018b; Jasper 2014) and four studies as having a high risk of bias with regard to the likely impact that unblinded outcome assessment had on the study results (Beukes 2018a; Kreuzer 2012; Malinvaud 2016; Schmidt 2018). We rated Beukes 2018b as low risk of bias as the data analysts were blinded to group allocation. Jasper 2014 specifically investigated the perceived expectations and credibility of the intervention (iCBT) and control (online discussion forum), and concluded that there was no effect of these on the results. The four studies rated as high risk of bias due to lack of blinding all reported that outcome assessors were not blinded. The remaining 22 studies did not report sufficient detail to judge whether outcome assessors were blinded and so we rated them as having unclear risk of detection bias.

\section{Incomplete outcome data}

We rated three studies as having a high risk (Abbott 2009; Davies 1995; Malinvaud 2016), 12 an unclear risk (Andersson 2002; Arif 2017; Henry 1998; Jakes 1986; Jakes 1992; Kreuzer 2012; Martz 2018; McKenna 2017; Nyenhuis 2013a; Oron (unpublished); Robinson 2008; Westin 2011), and 13 a low risk of attrition 
bias (Andersson 2005; Beukes 2018a; Beukes 2018b; Cima 2012; Henry 1996; Hesser 2012; Jasper 2014; Kaldo 2007; Lindberg 1989; Philippot 2012a; Schmidt 2018; Weise 2016; Zhong 2014).

Abbott 2009 reported a high level of attrition, most of which was unexplained, and replaced missing data with the last (observed) outcome carried forward. Davies 1995 reported differing levels of attrition across groups and concluded from this that the "differential attrition to some extent invalidates the group comparison". Malinvaud 2016 reported that different reasons were given by participants for dropout depending on the group they were allocated to, and missing data appear to be related to treatment outcome. Hence we also rated this study as having a high risk of attrition bias.

\section{Selective reporting}

We judged eight studies to be at high risk of reporting bias. These included studies with discrepancies between what was reported in the protocol or methods and what was published in the results (Abbott 2009; Jakes 1992; Malinvaud 2016; Martz 2018; Robinson 2008; Weise 2016). For example, Schmidt 2018 specified a primary outcome in the study protocol but did not report it as such in the publication, and additional outcomes not identified in protocol were reported in the publication. Three studies did not report enough information to judge the risk of reporting bias (Jasper 2014; Lindberg 1989; Zhong 2014), and one is currently being prepared for publication and hence we judged it as at unclear risk (Oron (unpublished)). We rated all other studies as low risk, having either fully reported what was specified in their respective methods sections, and/or reported everything as specified in a published protocol or trial registration (Arif 2017 Beukes 2018b; Cima 2012; Davies 1995; Henry 1996; Henry 1998; Hesser 2012; Kaldo 2007; Kreuzer 2012; McKenna 2017; Nyenhuis 2013a; Philippot 2012a; Westin 2011).

\section{Other potential sources of bias}

We judged two studies to be at risk of other biases. Martz 2018 did not explain why the initial anticipated sample size estimate was 80 but only 40 participants were included in the final publication, or why ACT was added as an intervention arm one year after initial trial registration. In Schmidt 2018 there were additional discrepancies between the protocol and publication, e.g. the protocol included a "no-intervention/standard care condition" but this was not reported in the publication of the study.

Four studies received an unclear rating for various reasons. In Jasper 2014 the inclusion criteria differed between the protocol (mini TQ score over 12) and study publications (mini TQ score over 8). In Kreuzer 2012 the long waiting time could lead to increased spontaneous improvements in the control group. Lindberg 1989 reported no scientific input from funders, but no protocol was available to inform our judgement. In Malinvaud 2016 there were some deviations from the protocol with an unknown effect on the outcomes of the study. Oron (unpublished) is not yet published. We judged all other studies as having a low risk of other biases.

\section{Effects of interventions}

See: Summary of findings for the main comparison CBT compared to no intervention/waiting list control for tinnitus; Summary of findings 2 CBT compared to audiological care (tinnitus education and rehabilitation for hearing loss) for tinnitus; Summary of findings 3 CBT compared to TRT (directive counselling and bilateral masking) for tinnitus; Summary of findings 4 CBT compared to other experimental control for tinnitus

\section{Comparison 1: Cognitive behavioural therapy (CBT) versus no} intervention/wait list control

\section{Primary outcomes}

\subsection{Impact of tinnitus on quality of life}

\subsubsection{End of treatment}

Ten studies reported data on the impact of tinnitus on quality of life at the end of treatment using validated multi-item questionnaires (Andersson 2002; Andersson 2005; Beukes 2018a; Henry 1996; Jakes 1992; Kaldo 2007; Kreuzer 2012; Malinvaud 2016; Oron (unpublished); Westin 2011). (Note: Post-treatment data from Malinvaud 2016 were not available and so, in accordance with a conservative approach, we entered baseline data showing no difference between the groups. This note applies to all analyses where Malinvaud 2016 is included). Overall, there was a clear difference in favour of CBT indicating that it may lead to a reduction in the impact of tinnitus on quality of life (standardised mean difference (SMD) - $0.56,95 \%$ confidence interval $(\mathrm{Cl})-0.83$ to -0.30 ; 10 studies; 537 participants; Analysis 1.1). Re-expressed as a score on the Tinnitus Handicap Inventory (THI; range 0 to 100) this is equivalent to a score 10.91 points lower in the CBT group, with an estimated minimal clinically important difference (MCID) for this scale being 7 points. A moderate level of heterogeneity was present $\left(\mathrm{Tau}^{2}=0.08 ; \mathrm{Chi}^{2}=17.62, \mathrm{df}=9(\mathrm{P}=0.04) ; \mathrm{I}^{2}=49 \%\right)$. The forest plot illustrating this result is shown in Figure 4 and it is reported in Summary of findings for the main comparison as a finding of importance. We rated the certainty of the evidence as low. 
Figure 4. Forest plot of comparison: 1 CBT versus no intervention/waiting list control, outcome: 1.1 Impact of tinnitus on quality of life at end of treatment.

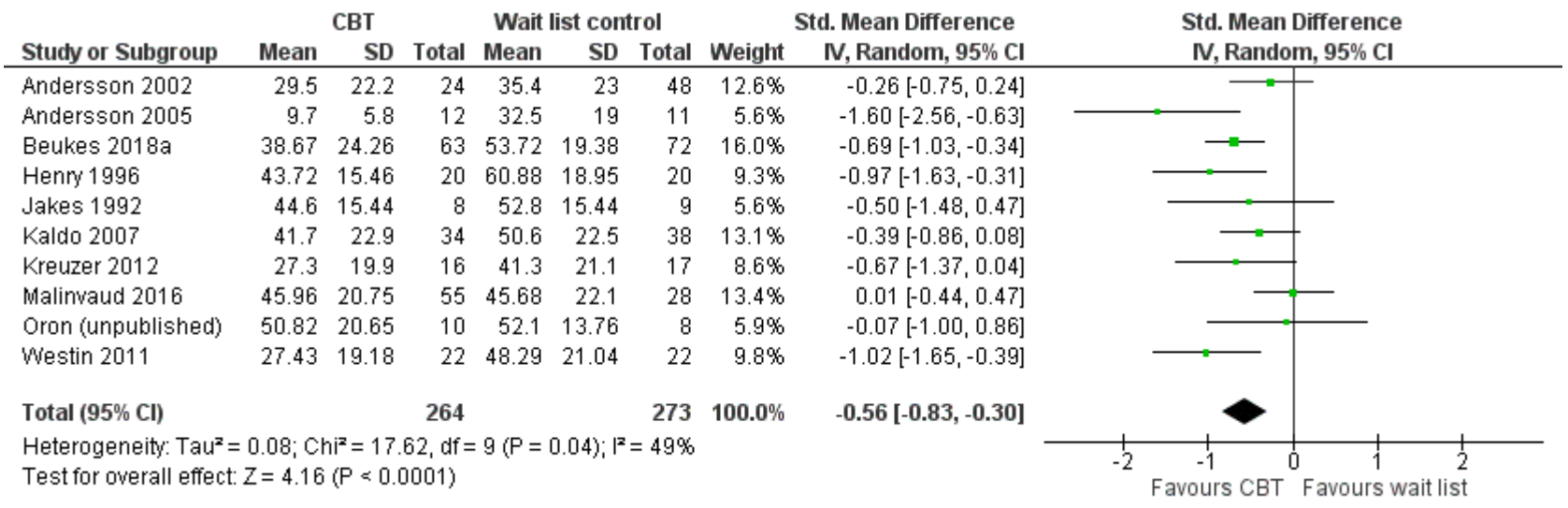

Subgroup analyses examining the type of therapy compared to wait list control indicated that there no statistically significant differences between the types of therapy, and that heterogeneity might not be important $\left(\mathrm{Chi}^{2}=1.80, \mathrm{df}=3(\mathrm{P}=0.61), \mathrm{I}^{2}=0 \%\right.$; Analysis 1.7): six studies used CBT (Andersson 2002; Andersson 2005; Beukes 2018a; Jakes 1992; Kaldo 2007; Malinvaud 2016), two used acceptance and commitment therapy (ACT) (Oron (unpublished); Westin 2011), one used cognitive therapy (Henry 1996), and one used mindfulness (Kreuzer 2012).

Subgroup analyses examining the mode of delivery (bibliotherapy, face-to-face and Internet-based) indicated that there were no significant differences between the modes of delivery, and that heterogeneity might not be important $\left(\mathrm{Chi}^{2}=0.69, \mathrm{df}=2(\mathrm{P}=0.71)\right.$, $\mathrm{I}^{2}=0 \%$; Analysis 1.9). Seven of the studies in this analysis delivered therapy face-to-face (Andersson 2005; Henry 1996; Jakes 1992; Kreuzer 2012; Malinvaud 2016; Oron (unpublished); Westin 2011), two via Internet interventions (Andersson 2002; Beukes 2018a), and one by bibliotherapy (Kaldo 2007).

When the unit of delivery was examined (i.e. individual compared with group), the subgroup analyses detected no significant differences and indicated that heterogeneity might not be a problem $\left(\mathrm{Chi}^{2}=0.01, \mathrm{df}=1(\mathrm{P}=0.94), \mathrm{I}^{2}=0 \%\right.$; Analysis 1.11): six studies delivered treatment in groups (Andersson 2005; Henry 1996; Jakes 1992; Kreuzer 2012; Malinvaud 2016; Oron (unpublished)), and four delivered it individually (Andersson 2002; Beukes 2018a; Kaldo 2007; Westin 2011).

Subgroup analyses by who delivered CBT (i.e. psychologists, "other clinician", computer, bibliotherapy) indicated no difference, and that heterogeneity might not be a problem $\left(\mathrm{Chi}^{2}=1.65\right.$, $\mathrm{df}=3(\mathrm{P}=0.65), \mathrm{I}^{2}=0 \%$; Analysis 1.13$)$ : four studies reported that psychologists delivered treatment (Henry 1996; Malinvaud 2016; Oron (unpublished); Westin 2011), three employed "other clinicians" (Andersson 2005; Jakes 1992; Kreuzer 2012), two used computers/Internet interventions (Andersson 2002; Beukes 2018a), and one used bibliotherapy (Kaldo 2007).

No data were available for subgroup analysis comparing selection of participants based on inclusion/exclusion criteria relating to severe hearing loss.

\subsubsection{Six months follow-up}

One study collected six-month follow-up data (Henry 1998), but by this time the participants in the wait list control group had received CBT and thus there was no comparison available.

\subsubsection{Twelve months follow-up}

Four studies collected 12-month follow-up data (Andersson 2002; Henry 1996; Kaldo 2007; Robinson 2008), but participants in the wait list groups had all received CBT treatment by that time and thus at the 12-month time point no comparison could be made.

\subsection{Serious adverse effects}

\subsubsection{End of treatment}

Seven studies, rated as moderate certainty, either reported or informed us via personal communication about serious adverse effects. Six informed us that no serious adverse effects occurred in the CBT or wait list condition at the end of treatment (Andersson 2002; Andersson 2005; Beukes 2018a; Kaldo 2007; Malinvaud 2016; Oron (unpublished)) and one study (Westin 2011) reported that one participant had deteriorated according to calculations of the reliable change index (Jacobson 1991) using the THI scores. This deterioration, however, occurred in the period between pretreatment assessment and the assessment following the first session but still appeared in the data at end of treatment. Given the timing of the deterioration, it is unlikely to be related to CBT (or specifically, ACT).

\subsubsection{Six months follow-up}

Westin 2011 reported at six-month follow-up that one participant of the 22 in the ACT condition had, according to reliable change calculations, deteriorated. This deterioration occurred in the period between pre-treatment assessment to the assessment following the first session (hence it was unlikely to be caused by CBT) but was still detectable at six-month follow-up.

\subsubsection{Twelve months follow-up}

At 12-month follow-up Beukes 2018c reported that three participants from the study Beukes 2018a developed "moderate" new symptoms, "moderate" negative well being, and that two participants had thought that treatment was too long. 


\section{Secondary outcomes}

\subsection{Depression}

\subsubsection{End of treatment}

Eight studies reported scores on measures of depression at the end of treatment (Andersson 2002; Andersson 2005; Beukes 2018a; Henry 1996; Kaldo 2007; Kreuzer 2012; Malinvaud 2016; Westin 2011). There was a statistically significant difference in favour of CBT (SMD - $0.34,95 \%$ Cl -0.60 to $-0.08 ; 8$ studies; 502 participants; Analysis 1.3) while moderate heterogeneity might be present ( $\mathrm{Tau}^{2}$ $\left.=0.06 ; \mathrm{Chi}^{2}=12.85, \mathrm{df}=7(\mathrm{P}=0.08) ; \mathrm{I}^{2}=46 \%\right)$. This result was reported in Summary of findings for the main comparison. Overall we rated the certainty of the evidence for this outcome as low.

\subsubsection{Six months follow-up}

No data were available for this outcome at this time point.

\subsubsection{Twelve months follow-up}

No data were available for this outcome at this time point.

\subsection{Anxiety}

\subsubsection{End of treatment}

Six studies reported scores on multi-item questionnaires measuring levels of anxiety at the end of treatment (Andersson 2002; Andersson 2005; Beukes 2018a; Kaldo 2007; Malinvaud 2016; Westin 2011). There was a statistically significant difference in favour of CBT (SMD $-0.45,95 \%$ Cl -0.82 to $-0.09 ; 6$ studies; 429 participants; Analysis 1.4). Substantial statistical heterogeneity was present $\left(\mathrm{Tau}^{2}=0.13 ; \mathrm{Chi}^{2}=15.36, \mathrm{df}=5(\mathrm{P}=0.009) ; \mathrm{I}^{2}=67 \%\right)$. We rated the certainty of the evidence for this outcome as very low meaning that the true effect is likely to be substantially different from the estimate of effect.

\subsubsection{Six months follow-up}

No data were available for this outcome at this time point

\subsubsection{Twelve months follow-up}

No data were available for this outcome at this time point.

\subsection{Health-related quality of life}

\subsubsection{End of treatment}

Two studies collected health-related quality of life data and the results indicated that there was a significant difference in favour of CBT (SMD $-0.38,95 \% \mathrm{Cl}-0.67$ to $-0.08 ; 2$ studies; 179 participants; Analysis 1.5). Heterogeneity was not likely to be important ( $\mathrm{Tau}^{2}$ $\left.=0.00 ; \mathrm{Chi}^{2}=0.26, \mathrm{df}=1(\mathrm{P}=0.61) ; \mathrm{I}^{2}=0 \%\right)$. Beukes 2018a used the Satisfaction with Life Scales and reported that the CBT group's quality of life improved significantly more than the wait list control group. Westin 2011 used the Quality of Life Index and linear mixed model regression to examine group by time effects over the 18month follow-up period of the study. Although means and standard deviations were reported at the end of the treatment phase for the groups (i.e. ACT, TRT and wait list control) the differences between the groups were not analysed at this time point.

We rated the certainty of the evidence as very low meaning that the true effect is likely to be substantially different from the estimate of effect.

\subsubsection{Six months follow-up}

No data were available for this outcome at this time point.

\subsubsection{Twelve months follow-up}

No data were available for this outcome at this time point.

\subsection{Negatively biased interpretations of tinnitus}

\subsubsection{End of treatment}

Two studies reported multi-item questionnaire measures of negatively biased interpretations of tinnitus (Henry 1996; Westin 2011). The analysis of the combined data revealed no difference between the CBT and wait list control groups. We rated the certainty of the evidence as very low, which suggests that the true effect is likely to be substantially different from the estimate of effect. There was high statistical heterogeneity $\left(I^{2}=73 \%\right)$, which could be explained by the different CBT interventions and units of delivery: Henry 1996 compared group cognitive therapy whereas Westin 2011 compared ACT delivered to individuals. Both studies, however, employed psychologists to provide the face-to-face intervention.

\subsubsection{Six months follow-up}

No data were available for this outcome at this time point.

\subsubsection{Twelve months follow-up}

No data were available for this outcome at this time point.

\subsection{Other adverse effects}

\subsubsection{End of treatment}

Seven studies, rated as moderate certainty, either reported or informed us via personal communication that no other adverse effects occurred in the CBT or wait list conditions at the end of treatment (Andersson 2002; Andersson 2005; Beukes 2018a; Kaldo 2007; Malinvaud 2016; Oron (unpublished); Westin 2011).

\subsubsection{Six months follow-up}

No data were available for this outcome at this time point.

\subsubsection{Twelve months follow-up}

No data were available for this outcome at this time point.

\section{Comparison 2: CBT versus usual audiological care}

Three studies compared CBT to audiological care (Beukes 2018b; Cima 2012; Schmidt 2018). No follow-up data were available at 6 or 12 months following the interventions. Two studies reported follow-up data at two months (Beukes 2018b; Schmidt 2018) and one at four months (Cima 2012).

We did not conduct subgroup analyses for this comparison as only three studies were included.

\section{Primary outcomes}

\subsection{Impact of tinnitus on quality of life}

\subsubsection{End of treatment}

Three studies each reported results using the THI and thus we pooled and analysed the data using mean differences (MD). The results indicated a statistically significant difference in favour of CBT compared to usual audiological care (MD -5.65, 95\% Cl -9.79 to -1.50 ; 3 studies; 430 participants; MCID = 7 points; Analysis 2.1). 
Statistical heterogeneity was unlikely to be important $\left(\mathrm{Tau}^{2}=0.00\right.$ $\left.\mathrm{Chi}^{2}=0.08, \mathrm{df}=2(\mathrm{P}=0.96) ; \mathrm{I}^{2}=0 \%\right)$. The forest plot is shown in Figure 5. We rated the certainty of the evidence as moderate reflecting that we are moderately confident that the true effect is likely to be close to the effect estimate but that there is a possibility that it could be substantially different (Summary of findings 2).

Figure 5. Forest plot of comparison: 2 CBT versus audiological care (tinnitus education and rehabilitation for hearing loss), outcome: 2.1 Impact of tinnitus on quality of life at end of treatment.

\begin{tabular}{|c|c|c|c|c|c|c|c|c|c|c|}
\hline \multirow[b]{2}{*}{ Study or Subgroup } & \multicolumn{3}{|c|}{ CBT } & \multicolumn{3}{|c|}{ Audiological care } & \multirow[b]{2}{*}{ Weight } & \multirow{2}{*}{$\begin{array}{l}\text { Mean Difference } \\
\text { IV, Random, } 95 \% \mathrm{Cl}\end{array}$} & \multirow{2}{*}{$\begin{array}{c}\text { Mean Difference } \\
\text { IV, Random, 95\% Cl }\end{array}$} & \\
\hline & Mean & SD & Total & Mean & SD & Total & & & & \\
\hline Beukes 2018b & 22.33 & 19.63 & 44 & 28.74 & 20.07 & 44 & $24.0 \%$ & $-6.41[-14.71,1.89]$ & $\longrightarrow=$ & \\
\hline Cima 2012 & 28.85 & 20.51 & 175 & 34.14 & 24.6 & 161 & $69.9 \%$ & $-5.29[-10.16,-0.42]$ & & \\
\hline Schmidt 2018 & 37.9 & 16.9 & 11 & 45.1 & 20 & 9 & $6.1 \%$ & $-7.20[-23.65,9.25]$ & & \\
\hline Total $(95 \% \mathrm{Cl})$ & & & 230 & & & 214 & $100.0 \%$ & $-5.68[-9.74,-1.61]$ & & \\
\hline \multicolumn{7}{|c|}{$\begin{array}{l}\text { Heterogeneity: } \text { Tau }^{2}=0.00 ; \mathrm{Chi}^{2}=0.09, \mathrm{df}=2(\mathrm{P}=0.96) ; \mathrm{I}^{2}=0 \% \\
\text { Test for overall effect: } Z=2.74(P=0.006)\end{array}$} & & & $\begin{array}{cccc}-20 & -10 & 0 & 10 \\
\text { Favours } & \text { CBT } & \text { Favours a }\end{array}$ & $\begin{array}{c}10 \\
20 \\
\text { udiol }\end{array}$ \\
\hline
\end{tabular}

\subsubsection{Six months follow-up}

No data were available for this outcome at this time point.

\subsubsection{Twelve months follow-up}

No data were available for this outcome at this time point.

\subsection{Serious adverse effects}

\subsubsection{End of treatment}

Two studies reported that no serious adverse effects occurred as a result of the intervention (Beukes 2018b; Cima 2012), and one study did not report information related to this outcome (Schmidt 2018). We rated the certainty of the evidence as moderate for this outcome (Summary of findings 2).

\subsubsection{Six months follow-up}

No data were available for this outcome at this time point.

\subsubsection{Twelve months follow-up}

No data were available for this outcome at this time point.

\section{Secondary outcomes}

\subsection{Depression}

\subsubsection{End of treatment}

Beukes 2018b used the PHQ-9 and Cima 2012 used the HADS$D$ to measure depression on multi-item questionnaires. The results indicated that there was no difference between CBT and audiological care (Analysis 2.3). We rated the certainty of the evidence as low suggesting that the true effect is likely to be different from the estimate of the effect (Summary of findings 2).

\subsubsection{Six months follow-up}

No data were available for this outcome at this time point.

\subsubsection{Twelve months follow-up}

No data were available for this outcome at this time point.

\subsection{Anxiety}

\subsubsection{End of treatment}

Beukes 2018b used the Generalised Anxiety Disorder-7 (GAD-7) scale and Cima 2012 the Hospital Anxiety and Depression Scale - Anxiety subscale (HADS-A) to measure anxiety on multi-item questionnaires. The results indicated that there was no difference between CBT and audiological care (Analysis 2.4). We rated the certainty of the evidence as low suggesting that the true effect is likely to be different from the estimate of the effect (Summary of findings 2).

\subsubsection{Six months follow-up}

No data were available for this outcome at this time point.

\subsubsection{Twelve months follow-up}

No data were available for this outcome at this time point.

\subsection{Health-related quality of life}

\subsubsection{End of treatment}

Beukes 2018b used the Satisfaction with Life Scale (SLWS) and Cima 2012 used the Health Utilities Index (HUI) to measure quality of life on multi-item questionnaires. The results indicated that there was no difference between CBT and audiological care (Analysis 2.5). We rated the certainty of the evidence as low, suggesting that the true effect is likely to be different from the estimate of the effect (Summary of findings 2).

\subsubsection{Six months follow-up}

No data were available for this outcome at this time point.

\subsubsection{Twelve months follow-up}

No data were available for this outcome at this time point.

\subsection{Negatively biased interpretations of tinnitus}

\subsubsection{End of treatment}

Cima 2012 used the Tinnitus Catastrophizing Scale to measure negatively biased interpretations of tinnitus. At the end of treatment there was a statistically significant difference in favour of CBT based on an intention-to-treat analysis (group difference $-4.683,95 \% \mathrm{Cl}-6.938$ to $-2.428, \mathrm{P}<0.0001$ ), which equated to a moderate effect size (Cohen's $d=0.60$ ). We rated the certainty of the evidence as low, suggesting that the true effect is likely to be different from the estimate of the effect (Summary of findings 2).

\subsubsection{Six months follow-up}

No data were available for this outcome at this time point. 


\subsubsection{Twelve months follow-up}

No data were available for this outcome at this time point.

\subsection{Other adverse effects}

No other adverse effects were reported at the end of treatment or at 6 and 12 months respectively in any of the three studies that compared CBT to usual audiological care. We rated the certainty of the evidence as moderate for this outcome (Summary of findings 2).

\section{Comparison 3: CBT versus tinnitus retraining therapy (TRT)}

One study compared a CBT intervention (ACT) with TRT and a wait list control condition (Westin 2011). For this comparison the wait list control group was included in the analyses reported in Comparison 1.

We did not conduct subgroup analyses for this comparison as only one study was included.

We assessed the certainty of the evidence for all the outcomes in this comparison as low (Summary of findings 3).

\section{Primary outcomes}

\subsection{Impact of tinnitus on quality of life}

Westin 2011 conducted linear mixed model analyses, which revealed a significant linear (time by group) interaction effect on the main outcome measure (THI) at all time points in the study (posttreatment, 6-month and 18-month follow-up; $\mathrm{F}(1,114)=8.49 ; \mathrm{P}=$ 0.04).

\subsubsection{End of treatment}

At end of treatment, there was a statistically significant difference in favour of ACT compared with TRT (MD $-15.79,95 \% \mathrm{Cl}-27.91$ to -3.67 ; 1 study; 42 participants; Analysis 3.1).

\subsubsection{Six months follow-up}

At six months follow-up, there was a statistically significant difference in favour of ACT compared with TRT (MD -13.10, 95\% Cl -26.08 to -0.12 ; 1 study; 42 participants Analysis 3.2).

\subsubsection{Twelve months follow-up}

No data were available for this outcome at this time point.

\subsection{Serious adverse effects}

\subsubsection{End of treatment}

Three participants deteriorated over the course of the study: one participant was in the ACT group $(n=22)$, and two participants were in the TRT group $(n=20)$. (See section 1.2.1 for information about the participant in the ACT group who deteriorated according to reliable change index calculations (Jacobson 1991; Westin 2011)).

\subsubsection{Six months follow-up}

The two participants in the TRT condition who deteriorated did so between 10 weeks and six months, although they also continued use of the wearable noise generators. (See section 1.2.2 for information about the participant in the ACT group who deteriorated (Westin 2011)).

\subsubsection{Twelve months follow-up}

No data were available for this outcome at this time point.

\section{Secondary outcomes}

\subsection{Depression}

\subsubsection{End of treatment}

At the end of treatment there were no time, group or interaction effects on depression scores as measured by the HADS-D (Westin 2011).

\subsubsection{Six months follow-up}

At six months follow-up there were no time, group or interaction effects on depression scores as measured by the HADS-D (Westin 2011).

\subsubsection{Twelve months follow-up}

No data were available for this outcome at this time point.

\subsection{Anxiety}

\subsubsection{End of treatment}

At the end of treatment there were no time, group or interaction effects on anxiety scores as measured by the HADS-A (Westin 2011).

\subsubsection{Six months follow-up}

At six months after treatment there were no time, group or interaction effects on anxiety scores as measured by the HADS-A (Westin 2011).

\subsubsection{Twelve months follow-up}

No data were available for this outcome at this time point.

\subsection{Health-related quality of life}

\subsubsection{End of treatment}

At the end of treatment there were no time, group or interaction effects on health-related quality of life scores as measured by the Quality of Life Inventory (QOLI) (Westin 2011).

\subsubsection{Six months follow-up}

At six months after treatment there were no time, group or interaction effects on health-related quality of life scores as measured by the QOLI (Westin 2011)

\subsubsection{Twelve months follow-up}

No data were available for this outcome at this time point.

\subsection{Negatively biased interpretations of tinnitus}

Westin 2011 used the Tinnitus Acceptance Questionnaire (TAQ) as a process measure rather than an outcome measure. Before treatment no differences were found between the three groups (ACT, TRT, wait list) concerning tinnitus acceptance $F(2,61)=1.21$; $P$ $=0.31$ (higher scores indicate better levels of tinnitus acceptance).

\subsubsection{End of treatment}

At the end of treatment there was a clear difference in favour of CBT compared with TRT (MD $-9.78,95 \% \mathrm{Cl}-16.40$ to $-3.16 ; 1$ study; 42 participants; Analysis 3.4). 


\subsubsection{Six months follow-up}

At 6 months follow-up, there was a difference in favour of CBT compared with TRT (MD -8.28, 95\% Cl -15.34 to -1.22 ; 1 study; 42 participants; Analysis 3.5).

\subsubsection{Twelve months follow-up}

No data were available for this outcome at this time point.

\subsection{Other adverse effects}

\subsubsection{End of treatment}

No data were available for this outcome at this time point.

\subsubsection{Six months follow-up}

No data were available for this outcome at this time point.

\subsubsection{Twelve months follow-up}

No data were available for this outcome at this time point.

\section{Comparison 4: CBT versus other active control}

Sixteen studies compared CBT to an active experimental control group not otherwise included in the previous comparisons (Abbott 2009; Arif 2017; Davies 1995; Henry 1996; Hesser 2012; Jakes 1986; Jakes 1992; Jasper 2014; Malinvaud 2016; Martz 2018; McKenna 2017; Nyenhuis 2013a; Oron (unpublished); Philippot 2012a; Weise
2016; Zhong 2014). Four of these studies did not provide any data that could be included in meta-analyses (Jakes 1986; Martz 2018; Philippot 2012a; Zhong 2014). For a description of the types of active comparison conditions used see Description of studies.

Abbott 2009 used a cluster-randomised controlled trial design but did not report the intracluster correlation coefficients and did not take into consideration the clustered nature of the data in their analyses. In consultation with a statistician, we obtained an estimate of an appropriate intracluster correlation coefficient from Meijerink 2017 and used this to adjust the sample size for the study.

\section{Primary outcomes}

\subsection{Impact of tinnitus on quality of life}

\subsubsection{End of treatment}

Twelve studies provided data on the impact of tinnitus on quality of life using multi-item questionnaires. Analysis indicated that the results were clearly in favour of $C B T$ regardless of what the active control condition was (SMD $-0.30,95 \% \mathrm{Cl}-0.55$ to $-0.05 ; 12$ studies; 966 participants; Analysis 4.1). The forest plot is shown in Figure 6 . Overall we rated the certainty of the evidence as low, which indicates that the true effect might be substantially different from the effect estimate (Summary of findings 4). Heterogeneity was moderate to substantial $\left(\mathrm{Tau}^{2}=0.12 ; \mathrm{Chi}^{2}=33.27, \mathrm{df}=11(\mathrm{P}=\right.$ 0.0005), $\left.I^{2}=67 \%\right)$.

Figure 6. Forest plot of comparison: 4 CBT versus other experimental control, outcome: 4.1 Impact of tinnitus on quality of life.

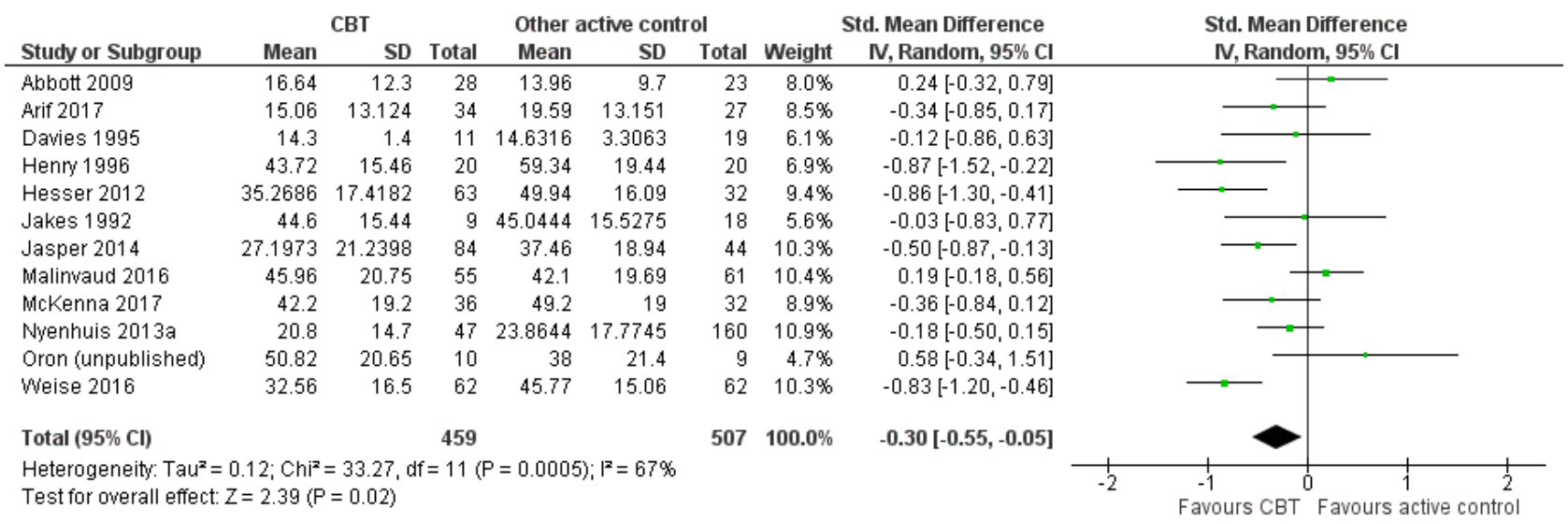

Subgroup analysis examining the type of therapy indicated that there were no significant differences between the types of therapy, and that heterogeneity was not a problem $\left(\mathrm{Chi}^{2}=0.29, \mathrm{df}=3 ; \mathrm{P}=\right.$ $0.96 ; 1^{2}=0 \%$; Analysis 4.14).

Subgroup analysis comparing face-to-face delivery to Internetbased delivery of CBT indicated that there was no significant difference between the respective modes of delivery $\left(\mathrm{Chi}^{2}=1.54 \mathrm{df}\right.$ $=1(P=0.21), I^{2}=35 \%$; Analysis 4.16)

Subgroup analysis examining whether there was a difference between $\mathrm{CBT}$ delivered in groups compared to individually found no difference $\left(\mathrm{Chi}^{2}=1.328, \mathrm{df}=1 ; \mathrm{P}=0.25 ; \mathrm{I}^{2}=24 \%\right.$; Analysis 4.17).
There were no significant group differences regarding who delivers the CBT (psychologists, Internet-based or "therapists") $\left(\mathrm{Chi}^{2}=0.15\right.$, $\mathrm{df}=2 ; \mathrm{P}=0.93 ; \mathrm{I}^{2}=0 \%$; Analysis 4.18).

No data were available for subgroup analysis comparing selection of participants based on inclusion/exclusion criteria relating to severe hearing loss.

\subsubsection{Six months follow-up}

McKenna 2017 reported that at six months after treatment there was a statistically significant difference in favour of CBT compared to relaxation therapy in the impact of tinnitus on quality of life, as measured by the Tinnitus Questionnaire (TQ). Specifically, the adjusted mean score in Mindfulness Based Cognitive Therapy (MBCT) $($ mean $=23, \mathrm{SD}-18.1)$ was 7.2 points lower $(95 \% \mathrm{Cl} 2.1-12.3$, 
$P=0.006)$ than in relaxation therapy (mean $=35.6, S D=16.8)$, with a standardised effect size of 0.56 ( $95 \% \mathrm{Cl} 0.16$ to 0.96$)$.

\subsubsection{Twelve months follow-up}

One study provided 12-month follow-up data comparing cognitive therapy combined with education to education only, on the impact of tinnitus on quality of life, as measured by the THQ (Henry 1996). Results from repeated measures ANOVAs indicated that there was no significant difference between the conditions.

\subsection{Serious adverse effects}

\subsubsection{End of treatment}

Nyenhuis 2013a reported that three participants deteriorated using the scores from the TQ in reliable change index calculations (Jacobson 1991); one participant was from the group CBT intervention and two from the information only control. We assessed the certainty of the evidence as low, meaning that the true effect could be substantially different from the effect estimate (Summary of findings 4).

\subsubsection{Six months follow-up}

No data were available for this outcome at this time point.

\subsubsection{Twelve months follow-up}

Weise 2016 reported that three participants showed "reliable deterioration" using the reliable change index for measures of the impact of tinnitus on quality of life (THI, $n=2$; Mini-TQ, $n=1$ ). (Note: by this time point there were no longer comparator participants since they had been offered and received CBT).

\section{Secondary outcomes}

\subsection{Depression}

\subsubsection{End of treatment}

Eleven studies supplied data for this analysis (Abbott 2009; Arif 2017; Davies 1995; Henry 1996; Hesser 2012; Jasper 2014; Malinvaud 2016; McKenna 2017; Nyenhuis 2013a; Philippot 2012a; Weise 2016). The results indicated a small difference in favour of CBT over other active interventions (SMD $-0.17,95 \% \mathrm{Cl}-0.33$ to -0.01 ; 11 studies; 943 participants; Analysis 4.5), and heterogeneity was unlikely to be important $\left(\mathrm{Chi}^{2}=13.00, \mathrm{df}=10 ; \mathrm{P}=0.22 ; \mathrm{I}^{2}=23 \%\right)$. We assessed the certainty of the evidence as low, meaning that the true effect could be substantially different from the effect estimate (Summary of findings 4).

\subsubsection{Six months follow-up}

At six months follow-up, McKenna 2017 reported that there were no differences between the MBCT group and active control (relaxation therapy) in HADS-D scores after pre-treatment scores had been taken into consideration (MD $-1.90,95 \% \mathrm{Cl}-3.87$ to $0.07 ; 1$ study; 62 participants; Analysis 4.6).

\subsubsection{Twelve months follow-up}

Henry 1996 compared cognitive therapy combined with education to an education only control condition, and reported that there was no significant difference between the groups (MD - 2.00, 95\% Cl-7.88 to 3.88 ; 1 study; 33 participants; Analysis 4.7).

\subsection{Anxiety}

\subsubsection{End of treatment}

Nine studies supplied data for the comparison of CBT to an active control (Abbott 2009; Arif 2017; Davies 1995; Hesser 2012; Jasper 2014; Malinvaud 2016; McKenna 2017; Philippot 2012a; Weise 2016). There was a significant difference in favour of CBT (SMD - 0.25 , $95 \% \mathrm{Cl}-0.48$ to $-0.02 ; 9$ studies; 696 participants; Analysis 4.8) with moderate to substantial heterogeneity $\left(\mathrm{Chi}^{2}=16.54, \mathrm{df}=8 ; \mathrm{P}=\right.$ $\left.0.04 ; I^{2}=52 \%\right)$. We rated the certainty of the evidence as low, which indicated that the true effect might be substantially different from the effect estimate (Summary of findings 4).

\subsubsection{Six months follow-up}

At six months follow-up, McKenna 2017 reported that there were no differences between the MBCT group and active control (relaxation therapy) in HADS-A scores after pre-treatment scores had been taken into consideration (MD $-1.20,95 \% \mathrm{Cl}-3.07$ to $0.67 ; 1$ study; 62 participants; Analysis 4.9).

\subsubsection{Twelve months follow-up}

No data were available for this outcome at this time point.

\subsection{Health-related quality of life}

\subsubsection{End of treatment}

One study reported quality of life data for the comparison of CBT and other active control (Hesser 2012), but found no difference between the conditions ( $\mathrm{MD}-0.05,95 \% \mathrm{Cl}-0.68$ to $0.59 ; 1$ study, 95 participants; Analysis 4.10). We rated the certainty of the evidence for this outcome as very low, reflecting that we have little confidence in the effect estimate and that the true effect is likely to be substantially different (Summary of findings 4).

\subsubsection{Six months follow-up}

No data were available for this outcome at this time point.

\subsubsection{Twelve months follow-up}

No data were available for this outcome at this time point.

\subsection{Negatively biased interpretations of tinnitus}

\subsubsection{End of treatment}

Data were supplied from five studies (Henry 1996; Hesser 2012; Jasper 2014; McKenna 2017; Weise 2016). These yielded results that found a significant difference in favour of CBT at the end of treatment (SMD $-0.55,95 \% \mathrm{Cl}-0.75$ to $-0.35 ; 5$ studies; 455 participants; Analysis 4.11). Heterogeneity might not be important $\left(\mathrm{Tau}^{2}=0.00 ; \mathrm{Chi}^{2}=4.23, \mathrm{df}=4(\mathrm{P}=0.38) ; \mathrm{I}^{2}=5 \%\right)$. We rated the certainty of the evidence for this outcome as moderate, indicating that the true effect is likely to be close to the effect estimate, but could possibly be substantially different (Summary of findings 4).

\subsubsection{Six months follow-up}

At six months follow-up, McKenna 2017 reported that there was a statistically significant difference between participants' scores in the MBCT group compared to the active control (relaxation therapy) on catastrophising as measured by the Tinnitus Catastrophizing Scale (TCS) (MD $-7.20,95 \% \mathrm{Cl}-13.65$ to $-0.75 ; 1$ study; 62 participants; Analysis 4.12). McKenna 2017 reported an adjusted mean difference of $-4.6(95 \% \mathrm{Cl}-8.7$ to -0.5$)$. 


\subsubsection{Twelve months follow-up}

One study provided 12-month follow-up data that compared cognitive therapy combined with education, to education only, on the negatively biased interpretations of tinnitus as measured by the Tinnitus Cognitions Questionnaire (Henry 1996). Results from repeated measures ANOVAs indicated that there were no significant differences between the conditions.

\subsection{Other adverse effects}

\subsubsection{End of treatment}

No other adverse effects were reported at end of treatment.

\subsubsection{Six months follow-up}

No data were available for this outcome at this time point.

\subsubsection{Twelve months follow-up}

One study reported some slight deterioration in sleep quality in three participants (Weise 2016). (Note: by this time point there were no longer comparator participants since they had been offered and received $\mathrm{CBT}$ ).

\section{Sensitivity analyses}

\section{Random-effects models compared to fixed-effect models}

There were no substantial differences between results depending on whether random-effects or fixed-effect modelling was used in the respective meta-analyses for the comparisons:

- CBT versus wait list control (Analysis 1.7; Analysis 1.8);

- CBT versus usual audiological care (Analysis 2.1; Analysis 2.7);

- CBT versus other active control (Analysis 4.14; Analysis 4.15).

\section{Excluding studies at high risk of bias for incomplete data}

Comparison 1: CBT versus no intervention/wait list control at end of treatment - impact of tinnitus on quality of life

We judged one study to be at high risk of bias for incomplete data (Malinvaud 2016). Retaining or excluding Malinvaud 2016 from the meta-analysis did not change the conclusion that CBT was more effective than wait list control, and did not change the effect size (moderate). With Malinvaud 2016 included the SMD was - 0.56 (95\% $\mathrm{Cl}-0.83$ to $-0.30 ; 10$ studies, 537 participants; Analysis 1.1) and with Malinvaud 2016 excluded the SMD was -0.64 ( $95 \% \mathrm{Cl}-0.88$ to -0.40 ; 9 studies, 454 participants; Analysis 1.15). Excluding Malinvaud 2016 from the analysis reduced the heterogeneity from I ${ }^{2}=49 \%\left(\mathrm{Tau}^{2}=\right.$ $\left.0.08 ; \mathrm{Chi}^{2}=17.62, \mathrm{df}=9(\mathrm{P}=0.04)\right)$ to $\mathrm{I}^{2}=28 \%\left(\mathrm{Tau}^{2}=0.04 ; \mathrm{Chi}^{2}=\right.$ $11.18, d f=8(P=0.19))$.

\section{Comparison 4: CBT versus other active control at the end of treatment} - impact of tinnitus on quality of life

We judged three studies to be at high risk of bias for incomplete outcome data (Abbott 2009; Davies 1995; Malinvaud 2016). With Abbott 2009, Davies 1995 and Malinvaud 2016 included the SMD was $-0.32(95 \% \mathrm{Cl}-0.56$ to $-0.08 ; 12$ studies; 967 participants; Analysis 4.1), therefore lower than with the studies excluded (SMD $-0.48,95 \% \mathrm{Cl}-0.71$ to -0.26 ; 9 studies; 770 participants; Analysis 4.21). Excluding the studies did not change the finding that CBT was more effective at decreasing the impact of tinnitus on quality of life, but did lead to a change in the effect size from 'small' to 'moderate'. Statistical heterogeneity was also reduced when the three studies were excluded, from $\mathrm{I}^{2}=66 \%\left(\mathrm{Tau}^{2}=0.11 ; \mathrm{Chi}^{2}=32.28, \mathrm{df}=11(\mathrm{P}=\right.$ $0.0007))$ to $\mathrm{I}^{2}=47 \%\left(\mathrm{Tau}^{2}=0.05 ; \mathrm{Chi}^{2}=15.18, \mathrm{df}=8(\mathrm{P}=0.06)\right)$.

Effects of replacing missing data with a 'conservative' compared with an 'optimistic' approach

One study that was included in two comparisons had missing data that were replaced with (baseline) data showing no difference between the groups (Malinvaud 2016). To examine the effect of entering data on a 'conservative' basis showing no effect, we undertook a sensitivity analysis replacing it with data at three months follow-up, which showed a change in participants' scores in favour of CBT.

Comparison 1: CBT versus no intervention/wait list control at the end of treatment - impact of tinnitus on quality of life

Using data showing a more 'optimistic' response at the end of treatment resulted in a small increase in the SMD but did not change the effect size (moderate) or conclusion that CBT was more effective than wait list control at reducing the impact of tinnitus on quality of life at the end of treatment. Specifically, using the 'conservative' approach, the SMD was $-0.56(95 \% \mathrm{Cl}-0.83$ to -0.30 ; Analysis 1.1) compared to the 'optimistic' scenario using threemonth follow-up data (SMD $-0.65,95 \% \mathrm{Cl}-0.85$ to -0.44 ; Analysis 1.16). Heterogeneity decreased from $\mathrm{I}^{2}=49 \%\left(\mathrm{Tau}^{2}=0.08 ; \mathrm{Chi}^{2}=\right.$ 17.62, $\mathrm{df}=9, \mathrm{P}=0.04)$ to $\mathrm{I}^{2}=20 \%\left(\mathrm{Tau}^{2}=0.02 ; \mathrm{Chi}^{2}=11.29, \mathrm{df}=9, \mathrm{P}\right.$ $=0.26$ ) with the use of three-month follow-up data.

\section{Comparison 4: CBT versus other active control at the end of treatment} - impact of tinnitus on quality of life

Using data that showed a more 'optimistic' response at the end of treatment resulted in a small increase in SMD score in favour of $\mathrm{CBT}$, but did not change the effect size (small). Specifically, the SMD increased from $-0.32(95 \% \mathrm{Cl}-0.56$ to -0.08 ; Analysis 4.1$)$ to -0.37 $(95 \% \mathrm{Cl}-0.58$ to -0.17 ; Analysis 4.23$)$ when the more 'optimistic' data were used. Furthermore, heterogeneity decreased from $\mathrm{I}^{2}=66 \%$ $\left(\mathrm{Tau}^{2}=0.11 ; \mathrm{Chi}^{2}=32.28, \mathrm{df}=11, \mathrm{P}=0.0007\right)$ to $\mathrm{I}^{2}=54 \%\left(\mathrm{Tau}^{2}=0.07\right.$; $\mathrm{Chi}^{2}=23.74, \mathrm{df}=11, \mathrm{P}=0.01$ ).

\section{DISCUSSION}

\section{Summary of main results}

The objective of this review was to assess the effects and safety of cognitive behavioural therapy (CBT) for tinnitus in adults. Twentyeight randomised controlled trials (RCTs) were included in the review with 21 of these supplying data for inclusion in metaanalyses. The four main comparisons of interest were wait list control, usual audiological care, tinnitus retraining therapy (TRT) and 'other' active control conditions.

Ten studies supplied data for the comparison of CBT with no intervention/wait list control. However, not all of them provided data on all the outcomes of interest for this review. There was evidence to indicate that CBT was superior to not providing any intervention in reducing the impact of tinnitus on quality of life and depression. However, there is limited evidence for CBT for tinnitus reducing anxiety, improving health-related quality of life, or reducing negatively biased interpretations of tinnitus (Summary of findings for the main comparison). Furthermore, information from six of the 10 studies indicated that there were no serious adverse effects at the end of treatment. One study reported that one participant who received CBT experienced a deterioration in 
their symptoms. Although the results appear promising, effect sizes were small or medium, and the certainty of the evidence was very low, low or moderate, depending on the specific outcome.

Three studies supplied data for the comparison of CBT with audiological care. With regard to reducing the impact of tinnitus on quality of life, there was evidence to indicate that CBT was superior to audiological care and without serious adverse effects. The mean difference across the three studies (5.65) was less than the difference of seven points that has been reported to reflect a clinically important change (Zeman 2011). For this comparison though it is relevant to consider that one large study $(n=336)$ reported a large effect in favour of CBT and influences the overall result. There were negligible differences between CBT and audiological care on measures of depression, anxiety or quality of life. There was insufficient evidence to conclude that either CBT or audiological care is superior or inferior with regard to reducing negatively biased interpretations of tinnitus. No other adverse effects occurred. (See Summary of findings 2).

There was insufficient evidence to conclude that CBT is superior or inferior to TRT on the outcomes of interest as only one study was included for this comparison (Westin 2011). In one of the few examples of studies reporting adverse effects, Westin 2011 found that three participants tinnitus worsened following treatment (one from the CBT and two from the TRT group). (See Summary of findings 3 ).

Twelve studies compared CBT to another active control condition. Across the studies there was variation in what form of CBT was used, what the comparison group received, whether it was individual or group treatment, delivered in person or not, and who delivered the treatment(s). Despite this, there was evidence to indicate that overall and albeit with small effect sizes, CBT is superior to other active treatments (e.g. relaxation, information sessions) in reducing the impact of tinnitus on quality of life. Similarly, small effect sizes in favour of CBT were found for reducing symptoms of depression, anxiety and negatively biased interpretations of tinnitus. Data from six of the 12 studies also suggest that adverse effects were less likely with CBT than with other active treatments. There was insufficient evidence to support the superiority or inferiority of CBT compared to other active treatments on quality of life. (See Summary of findings 4).

Sensitivity analyses indicated that the findings and conclusions were robust to tests of assumptions and the methods used.

\section{Results from subgroup analyses}

The subgroup analyses examined whether there were differences in effects between: the types of CBT; modes of delivery; unit of delivery; health professional involved in delivery; and whether effects differed between studies that excluded participants according to hearing thresholds. Results from each of these analyses are briefly discussed below.

\section{Type of CBT}

Subgroup analyses for both the comparison of CBT versus wait list control and an active control intervention (excluding usual audiological care and TRT) found no statistically significant differences between the types of CBT (Analysis 1.7; Analysis 4.14). It is important to note, however, that the most frequent intervention type included in the subgroup analyses was CBT.
In summary, the type of CBT intervention used might not matter, but a relatively low number of studies using mindfulness or acceptance and commitment therapy (ACT) (i.e. 'third wave' CBT) prevents more definitive conclusions from being drawn.

\section{Mode of delivery}

While there was no statistically significant difference between the effects of CBT when mode of delivery was taken into account, there was greater variation in effect size when CBT was delivered faceto-face compared with as an Internet-based intervention. If CBT delivered as an Internet-based intervention is indeed as effective as face-to-face CBT, this has implications for access to treatment and cost-effectiveness. (See Implications for practice).

\section{Unit of delivery}

Subgroup analyses in the respective comparisons examining whether there were difference in effectiveness of CBT delivered individually compared with groups were consistent in their results. They both found that there was no statistical difference when CBT was delivered individually compared to groups (Analysis 1.11; Analysis 4.17).

\section{Health professional involved in delivery}

There were no statistically significant differences in effect between who (or what) delivered CBT when comparing CBT to wait list (Analysis 1.13) or an 'other' active control (Analysis 4.18). Two issues are important to consider. First, the Internet-delivered and bibliotherapy CBT interventions were designed by psychologists but were not delivered by psychologists. Secondly, it was unclear exactly in what training or profession the "other clinicians" were in four studies across the two subgroup analyses (Andersson 2005; Arif 2017; Jakes 1992; Kreuzer 2012). These issues prevent conclusions being drawn with regard to the question of whether it matters who delivers CBT.

\section{Hearing thresholds as a selection criteria in RCTs of CBT for tinnitus}

Only one study included in this review excluded participants with severe hearing loss (Westin 2011). On one hand, this means that it is not possible to establish whether there are differences in results obtained between studies that examine whether there are differences in effectiveness of CBT depending on hearing loss, but on the other hand, it means that the results from the review can be applied to all people seeking help for tinnitus-related distress regardless of whether or not they have hearing loss.

\section{Additional results and considerations}

\section{Many articles did not report or collect data on adverse effects or other outcomes of interest}

Adverse effects of CBT for tinnitus are rare. However, this conclusion comes with the caveat that relatively few studies actually reported whether adverse effects occurred or not, and when they did there was little or no information on the method used to identify adverse effects. The exception to this being in four studies (Beukes 2018a; Nyenhuis 2013a; Weise 2016; Westin 2011), where deterioration on measures of tinnitus-related quality of life was established using the calculation of reliable change indices. When not reported, information about adverse effects was sought from and/or obtained from personal email communication with authors involved in the studies. Although a lot of authors responded to our 
inquiries, not all were able to recall whether there were adverse effects or not.

In addition to the absence of data relating to adverse effects, it was relatively rare for studies to collect outcome data for quality of life ( 6 of 28 studies) and negatively biased interpretations of tinnitus (10 of 28 studies).

\section{Few studies collected six- and 12-month follow-up data}

Collecting longer-term follow-up data is vital for examining whether or not any intervention effects are sustained over time. This in turn can have an effect on treatment preferences of the patient, service provider and at a policy level. The time points of interest for this review were post-treatment, six- and 12-month follow-up, which in the context of 'longer-term follow-up' is not actually that long. Unfortunately, only two studies reported outcome data that were collected at six months and six studies collected outcome data at 12 months follow-up. Even then, not all the data collected at these time points could be included in meta-analyses as the comparators were mostly wait list control conditions and participants had already received $\mathrm{CBT}$ by the six- or 12 -month time point. The average duration for which follow-up data were collected was four months and the median was three months.

Short-term follow-up data might also limit the ability of multiitem questionnaires to detect changes in overall quality of life in particular. This might be the case here because a change in one domain (e.g. tinnitus-related quality of life) might not immediately generalise or have an effect on other domains of life in the short term, but might do so in the longer term.

\section{Origin of CBT materials}

Viktor Kaldo and Gerhard Andersson co-authored a manual (in Swedish) for conducting CBT for tinnitus (Kaldo 2004a), which subsequently formed the basis for many bibliotherapy and Internet-based interventions. Nine of the 21 studies that supplied data for meta-analyses are derived from this work. In addition to the Swedish studies (Andersson 2002; Andersson 2005; Hesser 2012; Kaldo 2007), the treatment manual and Internet-based interventions have been adapted, translated and used in English(Abbott 2009; Beukes 2018a; Beukes 2018b) and German-speaking populations (Jasper 2014; Weise 2016). With the exception of Abbott 2009, which used a cluster-randomised control design and had a high participant dropout rate, results from the other eight studies were consistently in favour of CBT over comparison conditions. This suggests that the CBT content delivered with little human interaction is efficacious and robust to different contexts and languages.

\section{Issues of sample size}

With the exception of one study, all studies involved samples of fewer than 150 participants. Cima 2012 included 492 participants. This one large study reported a large effect size in favour of CBT and was weighted highly in the meta-analysis for the comparison in which it was included (CBT compared to audiological care). Ultimately the result showing that CBT was superior to audiological treatment was thus largely determined by the results of Cima 2012.

\section{Outlier study}

In general the results of studies included in this review found that $\mathrm{CBT}$ is superior to other control/comparator conditions with regard to reducing the impact tinnitus has on quality of life. The notable exception to this was Oron (unpublished), which found that the participants in the comparator intervention (coping effectiveness training; CET) had significantly lower scores on the THI than participants in the acceptance and commitment therapy (ACT) condition. A possible explanation for this result could be that CET appears to have some intervention components that are commonly found in CBT interventions. For example, CET included problemsolving skills with the intention of reducing stress, relaxation and pleasant activity scheduling.

\section{Clinical significance}

All of the included studies report group-level statistical significance of changes in the outcomes of the interventions. However, only 15 studies discussed clinical significance. Reporting the clinical significance of an intervention is important in order to provide an indication of size of effect, and the numbers of individual participants who might have benefited, remained the same or deteriorated following treatment. Clinical significance can be calculated in many ways and there is no standardised criteria or method for doing this. For example, within the included studies Kaldo 2007 used a pre-specified criteria of a 50\% reduction in the Tinnitus Reaction Questionnaire (TRQ) score as a sign of clinically significant change. In contrast, Weise 2016 used two methods to estimate clinical significance. Firstly, the Reliable Change Index (Jacobson 1991) to establish the change in score required to reflect change beyond measurement error, and second, calculating the numbers of participants who were "highly functioning" following treatment according to if they scored in the "mild" range on the THI (i.e. less than or equal to 36 ).

Given that there were only small to moderate effect sizes achieved, it seems that there is still room for improvement in the design and implementation of CBT interventions for tinnitus. However, by way of comparison, the magnitudes of the effect sizes reported in this review are similar to those reported for psychological interventions for treating chronic pain (Ecclestone 2013), which has many conceptual similarities to chronic tinnitus.

\section{Studies at high risk of bias for missing data affected heterogeneity}

Sensitivity analyses revealed that including studies judged to be at high risk for missing data inflated the level of heterogeneity (Abbott 2009; Davies 1995; Malinvaud 2016). Furthermore, for Comparison 1, the inclusion of Malinvaud 2016 also contributed to us downgrading the level of evidence for inconsistency in the results - that is, inconsistency that we introduced ourselves into the data set by including the study.

\section{Limitations}

The results of this review should be interpreted with five main limitations in mind. First, information about adverse effects associated with CBT was rarely reported, and the methods associated with monitoring or seeking information about adverse effects were not reported. Where information about adverse effects (serious or otherwise) was not reported, we made efforts to contact authors to obtain this information. Not every author we contacted responded to our requests for information and in many cases, information was dependent on recall of studies that occurred many years ago. It is conceivable that authors who responded to our inquiries were less likely to have observed adverse effects in their 
studies, thus leading to an underestimation of the risks associated with CBT. Nonetheless, CBT is considered to be a safe intervention, compared to surgical and pharmacological treatments since it is non-invasive.

A second limitation within this review concerns the lack of sixand 12-month follow-up data. Very few studies collected longerterm follow-up data, which in turn limits our ability to draw any conclusions about the longer-term efficacy of CBT for tinnitus. This has long been recognised as an issue within tinnitus research (Henry 1996), but little has changed in recent years.

'Risk of bias' assessments revealed that there were very few studies at low risk of performance and detection biases. This is partially a product of $\mathrm{CBT}$ as intervention being difficult to mask from participants and that trialists depended on multi-item self-report questionnaires of subjective variables such as tinnitus-related quality of life. None of the included studies had, for example, an independent outcome assessor to complement the responses obtained from the self-report questionnaires.

Another limitation within the review is the limited number of studies included for the subgroup analyses: those results should be interpreted with caution.

Lastly, although not an inherent limitation, we only included evidence from parallel-group and cluster-randomised controlled trials. Excluding non-randomised studies may limit the generalisability of drawing conclusions on how well CBT for tinnitus will work in everyday clinical practice. Although accompanied by higher risks of bias, the (potential) benefit of non-randomised trials is that they can inform judgments of the effectiveness of CBT when implemented in everyday practice.

\section{Overall completeness and applicability of evidence}

\section{Completeness}

The completeness of the evidence was variable with regard to the four main comparisons included in this review. There is sufficient evidence of moderate certainty to conclude that $C B T$ is superior to wait list control conditions at the end of treatment. However, there were too few studies comparing CBT with audiological care and TRT respectively to reach a conclusion. For the comparison of CBT with any other active intervention, the rating of the evidence (i.e. low certainty) means that our confidence in the effect estimate is limited.

Information about adverse effects (serious or otherwise) was rarely reported (although we obtained information about adverse effects from some authors). Three-quarters of the studies provided data for inclusion in meta-analyses of the primary outcome (impact of tinnitus on quality of life). Outcome data for depression and anxiety were more frequently reported than data related to quality of life and negatively biased interpretations of tinnitus.

Across all comparisons and outcomes there was an absence of evidence of the efficacy of CBT at 6 or 12 months after treatment.

For the subgroup analyses, there were considerable differences in the number of studies that provided data within the respective comparisons. For example, there was a comparable number of studies using different modes and units of delivery, but not of types of therapy, professional delivering therapy or of studies using and not using hearing loss as an exclusion criteria.

\section{Applicability}

There was a relatively complete representation of the population of people living with and seeking psychological help for tinnitus. There was a wide age range, a wide range of tinnitus duration, and a balance of males and females. Only one study excluded participants based on hearing thresholds (Westin 2011). The most frequent exclusion criteria were objective tinnitus and/or severe mental health conditions. In summary, the evidence appears applicable to people suffering from chronic tinnitus. People suffering from tinnitus and seeking pharmacological, electrical or electromagnetic stimulation therapy, or bio- neuro-feedback treatments would not have sought to participate in the included studies, and thus could be different in some characteristics.

Between the studies there was considerable variation in the possible combinations and permutations of $\mathrm{CBT}$; that is, there was variation in the type of $\mathrm{CBT}$, how it was delivered to participants, the unit of delivery, who delivered CBT and the duration of treatment. With the exception of the studies linked to Andersson and Kaldo's work there was also variation in the content of the CBT interventions included/used in the studies. The planned comparisons we specified meant that almost all RCTs of CBT for tinnitus have been included, making the results applicable to CBT for tinnitus in general.

The results from this review are most applicable to when either a psychologist (or psychologist in training) delivers the interventions and/or designed the CBT intervention. Although 'therapists' were referred to in some studies there was, overall, an absence of evidence from studies that had professionals other than psychologists delivering CBT. The absence of follow-up data also means that the results are only applicable to CBT in the short rather than medium or long term.

It is also important to recognise that the data included in this review came from studies conducted in outpatient clinics in a relatively small number of European countries, America and Australia. One study from China was included in the review, but did not provide data suitable for inclusion in meta-analyses. We are thus unclear about the effectiveness of CBT for tinnitus when conducted in inpatient settings or in other countries beyond those included here. Similarly, it is unclear what effects CBT might have when delivered by professionals such as audiologists due to an absence of data.

\section{Quality of the evidence}

For the comparison 'CBT versus no intervention/wait list control' the certainty of evidence for the two primary outcomes was low and moderate respectively. We downgraded the evidence (10 studies) by one level due to risk of bias associated with an absence of blinding of participants and personnel with regard to both the intervention and outcome assessment. We also downgraded the evidence for the outcome impact of tinnitus on quality of life by one level due to inconsistency in the results.

For the comparison 'CBT versus audiological care' the certainty of evidence for the primary outcomes was moderate. We downgraded the evidence (three studies) by one level due to risk of bias. We judged all studies in this comparison to be at high risk of 
performance bias due to an absence of blinding of participants and personnel.

For the comparison 'CBT versus tinnitus retraining therapy' we rated the certainty of evidence for the primary outcomes as low. We downgraded the evidence (one study) primarily due to high risk of bias associated with an absence of allocation concealment, and also an unclear risk of bias due to performance, detection and attrition biases. We also downgraded the evidence by one level due to imprecision (sample size fewer than 350 participants).

For the comparison 'CBT versus other experimental control' we rated the certainty of evidence for the primary outcomes as low. For the outcome of impact of tinnitus on quality of life we downgraded the evidence by two levels due to risk of bias (unclear or high risk associated with performance bias and blinding) and inconsistency (not all confidence levels overlapped and there was high statistical heterogeneity). For the outcome of serious adverse effects, we downgraded the evidence by two levels due to unclear or high risk of performance and attrition bias respectively.

\section{Potential biases in the review process}

The searches of the electronic databases were conducted independently of the group of authors by Information Specialists within the Cochrane ENT group. We also searched the reference lists of the included studies and previous Cochrane Reviews (MartinezDevesa 2010). Date of publication and language were not barriers to inclusion in this review. In addition to English, we reviewed full-text articles in Chinese and German for eligibility assessment. Where authors of this review were listed as authors of included studies, they were not involved in decisions related to screening, data extraction or risk of bias assessment.

We conscientiously followed Cochrane guidelines and the methods described in the protocol (Fuller 2017b). This, however, does not entirely prevent biases from influencing the outcome of the review. It is possible that biases influenced decisions in the development of the protocol and thus the procedures that we followed thereafter. For example, how we defined CBT determined the studies that were included. There is some debate about whether ACT and mindfulness constitute a new form of CBT or should be considered independently (Hofmann 2008). Similarly, after some debate within the group, we excluded Malouff 2010 on the grounds that giving participants a book based on CBT principles, but without any contact from a psychologist or other therapist, does not constitute CBT. It is possible that a different group of authors might have reached different conclusions about these issues.

There were three minor changes to the roles of authors in relation to data extraction and risk of bias assessments (see Differences between protocol and review). However, given that this led to additional participation from more rather than fewer authors, it is likely that biased thinking was decreased rather than increased as a result of greater scrutiny amongst the review team.

In this review, one cluster-randomised controlled trial was included (Abbott 2009). The analysis conducted by Abbott 2009 did not take into account the clustered nature of the data, and the corresponding author informed us that the data set was no longer available. The Cochrane Handbook for Systematic Reviews of Interventions advises that a post-hoc calculation can be made to adjust the sample size to account for the clustered nature of the data, using a value for the intracluster correlation (ICC) (Higgins 2011). If an ICC is not reported in the article, a suitable alternative should be identified. Ultimately, we used an ICC value from a cluster-RCT with hearing aid users as the best/closest approximation (Meijerink 2017). This ICC was identified by the Cochrane Methods Support Unit (see Acknowledgements) and the decision to use it was taken in consultation with the Cochrane ENT Managing Editor.

\section{Agreements and disagreements with other studies or reviews}

The conclusions of this review are mostly consistent with previous systematic reviews. The previous Cochrane Review of CBT for tinnitus concluded that "CBT has a positive effect on the management of tinnitus" (p.2; Martinez-Devesa 2010). This conclusion was based on results showing significant improvement in the impact of tinnitus on quality of life and depression scores. While we agree with this conclusion in principle, our results are more nuanced as a result of being able to include more recent studies and subsequently conduct subgroup analyses. Furthermore our conclusions about the efficacy of CBT are limited to post-treatment as there is an absence of evidence at six and 12 months follow-up.

The effect sizes for the impact of tinnitus on quality of life reported for CBT versus wait list control at end of treatment in MartinezDevesa 2010 were larger (standardised mean difference (SMD) 0.91, $95 \%$ confidence interval ( $\mathrm{Cl}) 0.50$ to $1.32 ; 5$ studies; 309 participants) than the ones we report (SMD $0.56,95 \% \mathrm{Cl}-0.83$ to $-0.30 ; 10$ studies; 537 participants). We identified a few examples of participant deterioration following CBT, though Martinez-Devesa 2010 did not.

Regarding the secondary outcomes, for depression our results for the comparison of CBT versus wait list control and versus another active treatment were in agreement. Specifically, each review reported medium and small effect sizes respectively. MartinezDevesa 2010, however, did not examine outcomes related to anxiety, quality of life or negatively biased interpretations of tinnitus. We on the other hand did not include subjective loudness of tinnitus as an outcome despite it being the primary outcome of Martinez-Devesa 2010. Research has consistently reported that CBT does not affect the perceptual characteristics of tinnitus.

While our conclusions are consistent with Martinez-Devesa 2010 there are differences in the studies that were included and excluded. We excluded the following studies that Martinez-Devesa 2010 included: Kröner-Herwig 1995; Kröner-Herwig 2003; Rief 2005; Weise 2008; Zachriat 2004. (See Excluded studies for reasons why the studies were excluded from this review). In contrast, we included the following studies that Martinez-Devesa 2010 excluded: Abbott 2009; Andersson 2002; Davies 1995; Henry 1998; Jakes 1986; Jakes 1992; Lindberg 1989; Robinson 2008. Five of these studies were excluded by Martinez-Devesa 2010 due to high participant dropout rates, a criterion that is no longer considered as valid for exclusion. One study was excluded for not having usable data (Henry 1998), one for not being randomised (Jakes 1986), and one study was judged not to have used CBT as an intervention (Lindberg 1989). We included these studies although none provided data that could be included in the meta-analyses.

Two studies were listed in Martinez-Devesa 2010 as ongoing studies at the time of publication. We included one, Schmidt 2018 (referred 
to as "Kendall 2009" in Martinez-Devesa 2010, p. 12) and excluded one, Zenner 2013 (referred to as "Zenner 2010" in Martinez-Devesa 2010, p. 12).

In addition to the previous Cochrane Review of CBT for tinnitus, there have been nine other systematic reviews that have examined the efficacy of CBT for tinnitus (Andersson 1999; Hesser 2011; Hoare 2011b; Nyenhuis 2013b; Cima 2014; Beukes 2019; Landry 2019; Mehta 2019; Rademaker 2019). Although these reviews differ from each other and our review in their focus and methodology, the conclusions are consistent in that CBT appears to be superior to other control conditions for alleviating the impact of tinnitus on quality of life. The moderate effect sizes for CBT versus wait list control reported by Hesser 2011 and Hoare 2011b are comparable with our results but smaller than the large effect size reported by Landry 2019.

\section{AUTHORS' CONCLUSIONS}

\section{Implications for practice}

There are six main implications for practice generated by this review:

1. The main results of this review indicate that cognitive behavioural therapy (CBT) may be effective in reducing the impact of tinnitus on quality of life at the end of treatment, and that there are few if any adverse effects from receiving CBT (although further research on this is recommended below). These results provide further evidence or justification for recommendations made in two prominent clinical guidelines endorsing the provision of CBT for patients with chronic bothersome tinnitus (Cima 2019; Tunkel 2014). Consequently, policy-makers and service providers should feel confident that CBT for tinnitus is beneficial for patients at least in the short term. This is not to say, however, that CBT is an easy form of treatment to engage in; it is often personally challenging and can require a considerable investment of time and money from the patient (assuming that CBT is even available and/or covered by insurance in a given country).

2. CBT for tinnitus appears to have some benefit for people who also experience depression, but the effects are small and there are some concerns with regards to the quality of the evidence. Thus, in addition to receiving tinnitus-specific CBT, people with co-morbid depression should also seek depression-specific treatment. Overall, there is either low-certainty evidence, small effects and/or an insufficient amount of evidence currently to recommend $C B T$ for tinnitus if the primary intention is to improve anxiety or general quality of life, or to change negatively biased interpretations of tinnitus.

3. CBT for tinnitus delivered in person and delivered via the Internet, with some additional email communication from a professional, appear similarly effective, as does CBT delivered individually and group-wise. Alternative modes of delivery should be considered depending on patient preference, accessibility and cost.

4. There is insufficient evidence to support a recommendation for whom should provide CBT for tinnitus, although it is noted that psychologists and/or psychiatrists were involved in the design, conduct and/or supervision of all CBT treatments.

5. The results from this review are relevant to tinnitus patients with varying levels of hearing loss and thus they should also be eligible to access treatment. We do not know, however, to what extent the study populations represent the whole patient population.

6. It is important to keep in mind that approximately half of the included studies in the review only reported group-level data/ analyses. This means that the results represent an average of the outcomes for participants in the study. In other words, on average, people improved receiving CBT compared with waiting for it (tinnitus) to get better, or an other available treatment. It is likely that individual patients might respond better or worse than the average treatment effects reported here and that patients should make informed choices aligned with personal preferences where possible.

\section{Implications for research}

Future research into the effectiveness of CBT for tinnitus should use the most rigorous methods available. Researchers can do this at least in part by using the SPIRIT statement when designing study protocols (Chan 2013), pre-registering trials at sites (such as clinicaltrials.gov, osf.io or aspredicted.org) and using the CONSORT statement to guide reporting of the results from the study (Schulz 2010). Using reporting guidelines and pre-registering trials can help ensure transparency in the conduct and reporting of studies and potentially increase confidence in the results and help future systematic reviewers make decisions with regard to whether a particular study meets eligibility criteria for inclusion. In this review only 10 of the 28 included studies were preregistered, only one protocol was published ahead of the study and one protocol fully published as a supplementary file. While the aforementioned regards the general planning and reporting of a study, researchers should in future make efforts to assess adverse effects, report how they did so and record whether adverse effects occurred. Researchers should also give greater consideration to assessment of outcomes and use complementary methods to selfreport questionnaires (e.g. blinded outcome assessors; healthcare use; multi-item self-report questionnaires completed by carers or partners of people receiving treatment). This would reduce some of the risks of bias associated with outcome assessment.

\section{Recommendations for future research include the following:}

1. Use follow-up measurement points of at least 6 to 12 months in order to assess the longer-term efficacy of CBT for tinnitus. Presently there is insufficient evidence available to comment on the efficacy of CBT at these time points. Researchers could also investigate the impact that 'booster' sessions might have in enhancing any of the effects from treatment and/or delaying or preventing relapse from occurring.

2. Use pre-specified primary outcomes including serious adverse effects. If multiple primary outcome measures are to be used, a rationale for doing so should be included. Ongoing work is currently being conducted to establish a (minimum) core set of outcome measures (Hall 2018), which should be included in all future studies in order to improve evidence synthesis and the ability to compare results between studies. We urge researchers to keep informed of future recommendations resulting from this work.

3. Systematically examine components of CBT interventions or compare specific CBT protocols, in order to reduce heterogeneity and further refine treatment protocols. 
4. Ensure that a power analysis is conducted to inform the target sample size for a study and report the results. If the target sample size is not reached this should be reported as well as a discussion on how this affected the power of the study and interpretation of results.

5. Examine the efficacy of CBT delivered by healthcare professionals other than psychologists (or trainee psychologists). If other health professionals can provide effective CBT for tinnitus, then there would be scope to increase access to treatment.

6. Establish which form of treatment works best for whom, especially since there is considerable heterogeneity in tinnitus. Randomised controlled trials (RCTs) are not typically designed to examine this issue and thus they would either need to be specifically designed to do so and/or other methods should be used. An alternative research method, which is increasingly used to inform such decisions, is the single case experimental design (Schork 2015). Single case experimental design studies have high internal validity and require fewer participants and resources to conduct.

7. Establish differences in effectiveness between types of CBT. This issue should be investigated in RCTs, single case experimental trials and future systematic reviews.

8. Ensure that estimates of clinical significance are included in the results of studies.

9. Incorporate treatment fidelity checks into RCTs to verify that interventions are delivered as intended. This is particularly relevant and important if the same therapists are delivering two or more interventions in the same study in order to assess if there is 'contamination' or insufficient 'protocol adherence' in the interventions.

\section{ACKN OWLEDGEMENTS}

We would like to thank the corresponding authors of the following included studies for providing additional information: Abbott 2009; Andersson 2002; Andersson 2005; Arif 2017; Beukes 2018a; Beukes 2018b; Cima 2012; Kaldo 2007; Lindberg 1989; Malinvaud 2016; Martz 2018; Oron (unpublished); Philippot 2012a; Robinson 2008; Schmidt 2018; Westin 2011. We would also like to thank the corresponding authors of Kröner-Herwig 1995, Kröner-Herwig 1999, Kröner-Herwig 2003, Kröner-Herwig 2006 and Tucker 2013, who provided additional information about their studies that ultimately helped inform our decisions on whether they met the inclusion criteria.

We would like to acknowledge Jenny Bellorini for all the practical assistance and guidance throughout the review. We thank Samantha Cox for preparing and conducting the initial and final literature searches, and Vittoria Lutje for conducting an update search. We would also like to thank Aidan Tan and Yu-Tian Xiao who extracted data from and conducted the risk of bias assessment for the Zhong 2014 study.

We would like to thank Nuala Livingstone and Kerry Dwan from the Cochrane Methods Support Unit for providing guidance and an intracluster correlation coefficient to enable data from Abbott 2009 to be included in meta-analyses.

Thomas Fuller was supported by SWOL Limburgs Fonds voor Revalidatie and the Netherlands Organisation for Health Research and Development (ZonMW), Research programme: Health Care Efficiency, Subprogramme: Effects \& Costs, Grant number: 945-07-715.

Rilana Cima received funding from the Innovational Research Incentives Scheme Veni, from the Netherlands Organisation for Scientific Research (NWO).

Derek J Hoare is funded through the National Institute for Health Research (NIHR) Biomedical Research Centre Programme. The view expressed are those of the author and not necessarily those of the NIHR, the NHS, or the Department of Health and Social Care.

Johan WS Vlaeyen received funding from the Research Foundation, Flanders FWO, Belgium (Fonds Wetenschappelijk Onderzoek Vlaanderen) and the Netherlands Organisation for Health Research and Development (ZonMW)

This project was supported by the National Institute for Health Research, via Cochrane Infrastructure, Cochrane Programme Grant or Cochrane Incentive funding to Cochrane ENT. The views and opinions expressed therein are those of the authors and do not necessarily reflect those of the Systematic Reviews Programme, NIHR, NHS or the Department of Health. 


\section{REFERE N CES}

\section{References to studies included in this review}

\section{Abbott 2009 \{published data only\}}

Abbott JA, Kaldo V, Klein B, Austin D, Hamilton C, Piterman L, et al. A cluster randomised trial of an internet-based intervention program for tinnitus distress in an industrial setting. Cognitive Behaviour Therapy 2009;38(3):162-73. [DOI: 10.1080/16506070902763174]

\section{Andersson 2002 \{published data only\}}

* Andersson G, Stromgren T, Strom L, Lyttkens L. Randomized controlled trial of internet-based cognitive behavior therapy for distress associated with tinnitus. Psychosomatic Medicine 2002;64(5):810-6. [DOI: 10.1097/01.PSY.0000031577.42041.F8]

\section{Andersson 2005 \{published data only\}}

* Andersson G, Porsaeus D, Wiklund M, Kaldo V, Larsen HC. Treatment of tinnitus in the elderly: a controlled trial of cognitive behavior therapy. International Journal of Audiology 2005;44(11):671-5. [DOI: 10.1080/14992020500266720]

\section{Arif 2017 \{published data only\}}

Arif M, Sadlier M, Rajenderkumar D, James J, Tahir T. A randomised controlled study of mindfulness meditation versus relaxation therapy in the management of tinnitus. Journal of Laryngology and Otology 2017;131(6):501-7. [DOI: 10.1017/ S002221511700069X]

Beukes 2018a \{published and unpublished data\}

* Beukes EW, Baguley DM, Allen PM, Manchaiah V, Andersson G. Audiologist- guided internet-based cognitive behavior therapy for adults with tinnitus in the United Kingdom: a randomized controlled trial. Ear and Hearing 2018; Vol. 39, issue 3:423-33. [0196-0202]

NCT02370810. A CBT-based internet intervention for adults with tinnitus in the United Kingdom [Study protocol for a CBT-based internet intervention for adults with tinnitus in the United Kingdom: a randomise controlled trial]. clinicaltrials.gov/show/ NCT02370810 (first received 25 February 2015). [CENTRAL: CN-01050683; CRS: 1781268]

\section{Beukes 2018b \{published data only\}}

* Beukes EW, Andersson G, Allen PM, Manchaiah V, Baguley D. Guided internet-based versus face-to-face clinical care in the treatment of tinnitus: a multicentre randomized noninferiority trial. JAMA Otolaryngology-Head \& Neck Surgery 2018;144(12):1126-33. [DOI: 10.1001/jamaoto.2018.2238]

NCT02665975. Internet-based versus face-to-face clinical care for tinnitus [Internet-based versus face-to-face clinical care for tinnitus: a multi-study randomised control trial]. clinicaltrials.gov/show/NCT02665975 (first received 28 January 2016). [CENTRAL: CN-01180255; CRS: 4390326]

Cima 2012 \{published and unpublished data\}

* Cima RF, Maes IH, Joore MA, Scheyen DJ, El Refaie A, Baguley DM, et al. Specialised treatment based on cognitive behaviour therapy versus usual care for tinnitus: a randomised controlled trial. Lancet 2012;379:1951-9.
NCT00733044. Cost-effectiveness of multidisciplinary management of tinnitus [Cost-effectiveness of multidisciplinary management of tinnitus at a specialised tinnitus centre]. clinicaltrials.gov/show/NCT00733044 (first received 12 August 2008). [CENTRAL: CN-00874706; CRS: 1648542]

Davies 1995 \{published data only\}

Davies S, McKenna L, Hallam RS. Relaxation and cognitive therapy: a controlled trial in chronic tinnitus. Psychology \& Health 1995;10(2):129-43.

Henry 1996 \{published data only\}

* Henry JL, Wilson PH. The psychological management of tinnitus: comparison of a combined cognitive educational program, education alone and a waiting-list control. International Tinnitus Journal 1996;2:9-20. [PUBMED: 10753339]

Henry 1998 \{published data only (unpublished sought but not used)\}

* Henry JL, Wilson PH. An evaluation of two types of cognitive intervention in the management of chronic tinnitus. Scandinavian Journal of Behaviour Therapy 1998;27(4):156-66. [DOI: 10.1080/02845719808408510]

Hesser 2012 \{published data only\}

Hesser H, Gustafsson T, Lunden C, Henrikson O, Fattahi K, Johnsson $\mathrm{E}$, et al. A randomized controlled trial of internetdelivered cognitive behavior therapy and acceptance and commitment therapy in the treatment of tinnitus. Journal of Consulting and Clinical Psychology 2012;80(4):649-61. [DOI: 10.1037/a0027021]

Jakes 1986 \{published data only\}

* Jakes S, Hallam R, Rachman S, Hinchcliffe R. The effects of reassurance relaxation training and distraction on chronic tinnitus sufferers. Behavior Research and Therapy 1986;24(5):497-507. [DOI: 10.1016/0005-7967(86)90029-X]

Jakes 1992 \{published data only\}

* Jakes SC, Hallam RS, McKenna L, Hinchcliffe R. Group cognitive therapy for medical patients - an application to tinnitus. Cognitive Therapy and Research 1992;16(1):67-82. [DOI: 10.1007/BF01172957]

\section{Jasper 2014 \{published data only\}}

* Jasper K, Weise C, Conrad I, Andersson G, Hiller W, Kleinstauber M. Internet-based guided self-help versus group cognitive behavioral therapy for chronic tinnitus: a randomized controlled trial. Psychotherapy and Psychosomatics 2014;83(4):234-46.

NCT01205906. Comparison of an internet-based guided selfhelp and a group therapy for chronic tinnitus [Comparison of the efficacy of an internet-based self-help training and a well-established outpatient group therapy for the treatment of chronic tinnitus: a randomized controlled trial]. clinicaltrials.gov/show/nct01205906 (first received 21 September 2010). [CENTRAL: CN-00921448; CRS: 1689266] 
Kaldo 2007 \{published data only\}

Kaldo V, Cars S, Rahnert M, Larsen HC, Andersson G. Use of a self-help book with weekly therapist contact to reduce tinnitus distress: a randomized controlled trial. Journal of Psychosomatic Research 2007;63(2):195-202.

\section{Kreuzer 2012 \{published data only\}}

Kreuzer PM, Goetz M, Holl M, Schecklmann M, Landgrebe M, Staudinger $S$, et al. Mindfulness-and body-psychotherapy-based group treatment of chronic tinnitus: a randomized controlled pilot study. BMC Complementary and Alternative Medicine 2012;12:235.

NCT01540357. Mindfulness-based therapy in chronic tinnitus [Mindfulness-based therapy for the treatment of chronic tinnitus: a randomized controlled pilot study]. clinicaltrials.gov/ show/NCT01540357 (first received 28 February 2012).

[CENTRAL: CN-00921453; CRS: 1689271]

Lindberg 1989 \{published data only (unpublished sought but not used)\}

Lindberg P, Scott B, Melin L, Lyttkens L. The psychological treatment of tinnitus: an experimental evaluation.

Behaviour Research and Therapy 1989;27(6):593-603. [DOI: 10.1016/0005-7967(89)90143-5]

Malinvaud 2016 \{published data only (unpublished sought but not used)\}

Malinvaud D, Londero A, Niarra R, Peignard P, Warusfel O, ViaudDelmon I, et al. Auditory and visual 3D virtual reality therapy as a new treatment for chronic subjective tinnitus: results of a randomized controlled trial. Hearing Research 2016;333:127-35. [DOI: 10.1016/j.heares.2015.12.023]

Martz 2018 \{published data only\}

Martz E, Chesney M, Livneh H, Jelleberg C, Fuller B, Henry JA. A pilot randomized clinical trial comparing three brief group interventions for individuals with tinnitus. Global Advances in Health and Medicine 2018;7:1-11.

NCT02293512. A comparison of three psychoeducational group interventions for veterans with tinnitus [A comparison of CBT and CET interventions for veterans with tinnitus]. clinicaltrials.gov/show/NCT02293512 (first received 18 November 2014). [CENTRAL: CN-01039743; CRS: 1770339]

\section{McKenna 2017 \{published data only\}}

McKenna L, Marks EM, Hallsworth CA, Schaette R. Mindfulnessbased cognitive therapy as a treatment for chronic tinnitus: a randomized controlled trial. Psychotherapy and Psychosomatics 2017;86(6):351-61. [0033-3190]

NCT02059447. A comparison of the benefits of mindfulness based cognitive therapy, relaxation therapy and a waiting list control in the management of distress in chronic tinnitus patients. clinicaltrials.gov/show/nct02059447 (first received 11 February 2014). [CENTRAL: CN-00994037; CRS: 1724717]

Nyenhuis 2013a \{published data only\}

Nyenhuis N, Zastrutzki S, Weise C, Jager B, Kroner-Herwig B. The efficacy of minimal contact interventions for acute tinnitus: a randomised controlled study. Cognitive Behaviour Therapy 2013;42(2):127-38. [DOI: 10.1080/16506073.2012.655305]

Oron (unpublished) \{unpublished data only\}

Martz E, Chesney MA, Livneh H, Ungar OJ, Harel S, Terracini D, Oron $Y$. Two brief group interventions for individuals with tinnitus in Israel. Unpublished.

NCT03068871. A comparison of two psycho-educational group interventions for tinnitus patients. https://clinicaltrials.gov/ show/nct03068871 (first received 3 March 2017). [CENTRAL: CN-01343644; CRS: 5479734]

\section{Philippot 2012a \{published data only\}}

Philippot P, Nef F, Clauw L, Romree M, Segal Z. A randomized controlled trial of mindfulness-based cognitive therapy for treating tinnitus. Clinical Psychology \& Psychotherapy 2012;19(5):411-9. [10.1002/cpp.756]

Robinson 2008 \{published data only\}

Robinson SK, Viirre ES, Bailey KA, Kindermann S, Minassian AL, Goldin PR, et al. A randomized controlled trial of cognitivebehavior therapy for tinnitus. International Tinnitus Journal 2008;14(2):119-26.

Schmidt 2018 \{published and unpublished data\} NCT00724152. Cognitive behavioral therapy (CBT) for tinnitus. clinicaltrials.gov/show/NCT00724152 (first received 29 July 2008). [CENTRAL: CN-00921437; CRS: 1689255]

* Schmidt CJ, Kerns RD, Finkel S, Michaelides E, Henry JA. Cognitive-behavioral therapy for veterans with tinnitus. Federal Practitioner 2018;35(8):36-46. [PUBMED: 30766380]

Weise 2016 \{published data only\}

NCT01205919. Internet-based guided self-help for chronic tinnitus [Efficacy of an internet-based guided self-help training for chronic tinnitus: a randomized controlled trial]. clinicaltrials.gov/show/NCT01205919 (first received 21 September 2010). [CENTRAL: CN-00921442; CRS: 1689260]

* Weise C, Kleinstauber M, Andersson G. Internet-delivered cognitive-behavior therapy for tinnitus: a randomized controlled trial. Psychosomatic medicine 2016;78(4):501-10.

\section{Westin 2011 \{published data only\}}

Westin VZ, Schulin M, Hesser H, Karlsson M, Noe RZ, Olofsson $\mathrm{U}$, et al. Acceptance and commitment therapy versus tinnitus retraining therapy in the treatment of tinnitus: a randomised controlled trial. Behaviour Research and Therapy 2011;49(11):737-47. [DOI: 10.1016/j.brat.2011.08.001]

Zhong 2014 \{published data only (unpublished sought but not used)\}

* Zhong C, Zhong Z, Luo Q, Qiu Y, Yang Q, Liu Y. The curative effect of cognitive behavior therapy for the treatment of chronic subjective tinnitus. Lin Chuang Er Bi Yan Hou Tou Jing Wai Ke za Zhi [Journal of Clinical Otorhinolaryngology, Head, and Neck Surgery] 2014;29(8):709-11. [PUBMED: 26248442] 


\section{References to studies excluded from this review}

Delb 2002 \{published data only\}

Delb W, D'Amelio R, Boisten CJ, Plinkert PK. Evaluation of the tinnitus retraining therapy as combined with a cognitive behavioral group therapy. HNO 2002;50(11):997-1004.

\section{Henry 2017 \{published data only\}}

Henry JA, Thielman EJ, Zaugg TL, Kaelin C, Schmidt CJ, Griest S, et al. Randomized controlled trial in clinical settings to evaluate effectiveness of coping skills education used with progressive tinnitus management. Journal of Speech, Language, and Hearing Research 2017;60(5):1378-97.

Kaldo 2008 \{published data only\}

* Kaldo V, Levin S, Widarsson J, Buhrman M, Larsen HC, Andersson $\mathrm{G}$. Internet versus group cognitive-behavioral treatment of distress associated with tinnitus: a randomized controlled trial. Behavior Therapy 2008;39(4):348-59.

\section{Konzag 2006 \{published data only\}}

Konzag TA, Rubler D, Bloching M, Bandemer-Greulich U, Fikentscher E, Frommer J. Counselling versus a self-help manual for tinnitus outpatients: a comparison of effectiveness. HNO 2006;54(8):599-604.

\section{Kröner-Herwig 1995 \{published data only\}}

Kröner-Herwig B, Hebing G, van Rijn-Kalkmann U, Frenzel A, Schilkowsky G, Esser G. The management of chronic tinnitus-comparison of a cognitive-behavioural group training with yoga. Journal of Psychosomatic Research 1995;39(2):153-65.

\section{Kröner-Herwig 1999 \{published data only\}}

Kroener-Herwig B, Esser G, Frenzel A, Fritsche G, Schilkowsky G. Result of an outpatient cognitive-behavioral group treatment for chronic tinnitus. Proceedings of the Sixth International Tinnitus Seminar, Cambridge, UK, 5-9 September, 1999. 1999:369-72.

\section{Kröner-Herwig 2003 \{published data only\}}

Kroner-Herwig B, Frenzel A, Fritsche G, Schilkowsky G, Esser G. The management of chronic tinnitus: comparison of an outpatient cognitive-behavioral group training to minimalcontact interventions. Journal of Psychosomatic Research 2003;54(4):381-9.

\section{Kröner-Herwig 2006 \{published data only\}}

Kroner-Herwig B, Zachriat C, Weigand D. Do patient characteristics predict outcome in the outpatient treatment of chronic tinnitus?. Psycho-social Medicine 2006;3:Doc07.

\section{Li 2019 \{published data only\}}

Juan L, Jianhua J, Songli X, Qian Z, Yuqin C, Min H, et al. Clinical efficacy of cognitive behavioral therapy for chronic subjective tinnitus. American Journal of Otolaryngology 2019;40:253-6.

\section{Luyten 2019 \{published data only\}}

Luyten T, Van de Heyning P, Jacquemin L, Van Looveren N, Declau F, Fransen E, et al. The value of Eye Movement Desensitization Reprocessing in the treatment of tinnitus: study protocol for a randomized controlled trial. Trials 2019;20:32.

\section{Malouff 2010 \{published data only\}}

Malouff JM, Noble W, Schutte NS, Bhullar N. The effectiveness of bibliotherapy in alleviating tinnitus-related distress. Journal of Psychosomatic Research 2010;68(3):245-51.

\section{Rief 2005 \{published data only\}}

Rief W, Weise C, Kley N, Martin A. Psychophysiologic treatment of chronic tinnitus: a randomized clinical trial. Psychosomatic Medicine 2005;67(5):833-8.

Scott 1985 \{published data only\}

Scott B, Lindberg P, Lyttkens L, Melin L. Psychological treatment of tinnitus. An experimental group study. Scandinavian Audiology 1985;14(4):223-30.

\section{Tan 2018 \{published data only\}}

Tan Y, Chen L, Luo Z, Ye W. The therapeutic effect of rehabilitation exercise and cognitive-behavioral intervention on the patients with tinnitus. Modern Clinical Nursing 2018;17(2):34-9.

\section{Tavakoli 2019 \{published data only\}}

Tavakoli M, Marashi S, Hamid N, Beshlideh K. The effects of cognitive-behavioral training and muscle relaxation on the degree of tinnitus and the quality of sleep. Auditory and Vestibular Research 2019;28(3):182-9.

Tucker 2013 \{published data only\}

Tucker EM. Tinnitus in Cochlear Implantees: Cognitive Behavioural Therapy for Cochlear Implant Users [Doctoral Thesis]. Available from: https://eprints.soton.ac.uk/. University of Southampton, Faculty of Engineering and the Environment, 2013.

Weise 2008 \{published data only\}

Weise C, Heinecke K, Rief W. Biofeedback-based behavioral treatment for chronic tinnitus: results of a randomized controlled trial. Journal of Consulting and Clinical Psychology 2008;76(6):1046-57.

\section{Zachriat 2004 \{published data only\}}

Zachriat C, Kroner-Herwig B. Treating chronic tinnitus: comparison of cognitive-behavioural and habituation-based treatments. Cognitive Behaviour Therapy 2004;33(4):187-98.

\section{Zenner 2013 \{published data only\}}

NCT00719940. Tinnitus treatment by structured cognitive behavioral therapy [Randomized controlled clinical trial of efficacy and safety of individual cognitive behavioral therapy (CBT) within the setting of the structured therapy program STCP (structured tinnitus care program) in patients with tinnitus aurium]. clinicaltrials.gov/show/nct00719940 (first received 22 July 2008). [CENTRAL: CN-00725092; CRS: 1526940]

Zenner HP, Vonthein R, Zenner B, Leuchtweis R, Plontke SK, Torka W, et al. Standardized tinnitus-specific individual cognitive-behavioral therapy: a controlled outcome study with 286 tinnitus patients. Hearing Research 2013;298:117-25. 
Zhicheng 2019 \{published data only\}

Zhicheng L. Resilience training to improve tinnitus distress: a randomized controlled trial. International Clinical Trials Registry 2019.

\section{References to ongoing studies \\ NCT03022084 \{published data only\}}

NCT03022084. Clinical trial of sound-based versus behavioral therapy for tinnitus. clinicaltrials.gov/show/NCT03022084 (first received 16 January 2017).

\section{NCT03386123 \{published data only\}}

NCT03386123. A comparison of CBTi and usual treatment for tinnitus related insomnia [A comparison of cognitive behaviour therapy for insomnia (CBTi) and usual audiological rehabilitation in the management of tinnitus related insomnia]. clinicaltrials.gov/show/NCT03386123 (first received 29 December 2017).

\section{NCT04004260 \{published data only\}}

Manchaiah V. CBT-based internet intervention for adults with tinnitus in the United States. https://clinicaltrials.gov/ct2/show/ nct04004260 (first received 2 July 2019).

\section{NTR6415 \{published data only\}}

NTR6415. Bold exposure or safe masking? A fear-conditioning approach to chronic tinnitus suffering [Bold exposure or safe masking? A fear-conditioning approach to chronic tinnitus suffering; a randomised controlled trial]. trialregister.nl/ trial/6235 (first received 24 April 2017).

\section{SLCTR/2018/005 \{published data only\}}

SLCTR/2018/005. Effectiveness of cognitive behavioral therapy for tinnitus [Effectiveness of modified cognitive behavioral therapy (CBT) in reducing distress for patients with tinnitus who are receiving treatment at two tertiary care hospitals in Sri Lanka, a randomised controlled trial]. slctr.lk/trials/ slctr-2018-005 (first received 2 February 2018).

\section{Additional references}

\section{Andersson 1999}

Andersson G, Lyttkens L. A meta-analytic review of psychological treatments for tinnitus. British Journal of Audiology 1999;33(4):201-10. [PUBMED: 10509855]

\section{Andersson 2001}

Andersson $\mathrm{G}$. The role of psychology in managing tinnitus: a cognitive behavioural approach. Seminars in Hearing 2001;22:65-76

\section{Andersson 2004}

Andersson G, Kaldo V. Internet-based cognitive behavioral therapy for tinnitus. Journal of Clinical Psychology 2004;60(2):171-8. [0021-9762: (Print)]

\section{Andersson 2009}

Andersson G. Tinnitus patients with cognitive problems: causes and possible treatments. The Hearing Journal 2009;62:27-8,30.

\section{Baguley 2013}

Baguley D, Andersson G, McFerran D, McKenna L. Tinnitus: A Multidisciplinary Approach. Second Edition. Chichester: John Wiley \& Sons, Ltd, 2013.

\section{Bakal 1972}

Bakal D. The Psychobiology of Chronic Headache. New York: Springer, 1972.

\section{Beck 1979}

Beck AT. Cognitive Therapy and the Emotional Disorders. New York: International Universities Press, 1979.

\section{Beck 1988}

Beck AT, Epstein N, Brown G, Steer RA. An inventory for measuring clinical anxiety: psychometric properties. Journal of Consulting and Clinical Psychology 1988;56(6):893-7.

\section{Beck 1996}

Beck AT, Steer RA, Brown GK. Manual for the Beck Depression Inventory-II. San Antonio, TX: Psychological Corporation, 1996.

\section{Bernstein 1973}

Bernstein DA, Borkovec TD. Progressive Relaxation Training: A Manual for the Helping Professions. Champaign, IL, US: Research Press, 1973:viii, 66.

\section{Bernstein 1984}

Bernstein DA, Borkovec TD. Progressive Relaxation Training. Champaign, Illinois: Research Press, 1984.

\section{Beukes 2018c}

Beukes E, Allen P, Baguley D, Manchaiah V, Andersson G. Longterm efficacy of audiologist-guided internet-based cognitive behavior therapy for tinnitus. American Journal of Audiology 2018;27:431-47. [DOI: 10.1044/2018_AJA-IMIA3-18-0004]

\section{Beukes 2019}

Beukes E, Manchaiah V, Allen P, Baguley D, Andersson G. Internet-based interventions for adults with hearing loss, tinnitus and vestibular disorders: A systematic review and metaanalysis. Trends in Hearing 2019;23:1-22.

\section{Biesinger 1998}

Biesinger E, Heiden C, Greimel V, Lendle T, Höing R, Albegger K. Strategien in der ambulaten Behandlung des Tinnitus. HNO 1998;46(2):157-69. [0017-6192]

\section{Bishop 2004}

Bishop SR, Lau M, Shapiro S, Carlson L, Anderson ND, Carmody J, et al. Mindfulness: a proposed operational definition. Clinical Psychology: Science and Practice 2004;11(3):230-41.

\section{Bouton 2007}

Bouton M. Learning and Behavior: A Contemporary Synthesis. Sunderland: MA Sinauer, 2007. 


\section{Buss 1998}

Buss E, Hall III JW, Grose JH, Hatch DR. Perceptual consequences of peripheral hearing loss: do edge effects exist for abrupt cochlear lesions?. Hearing Research 1998;125:98-108.

\section{Carver 1989}

Carver CS, Scheier MF, Weintraub JK. Assessing coping strategies: a theoretically based approach. Journal of Personality and Social Psychology 1989;56(2):267-83.

\section{Carver 1997}

Carver CS. You want to measure coping but your protocol's too long: consider the brief COPE. International Journal of Behavioral Medicine 1997;4(1):92-100.

\section{Chan 2013}

Chan A-W, Tetzlaff JM, Altman DG, Laupacis A, Gøtzsche PC, Krleža-Jerić K, et al. SPIRIT 2013 Statement: defining standard protocol items for clinical trials. Annals of Internal Medicine 2013;158(3):200-7.

\section{Cima 2011a}

Cima RF, Vlaeyen JW, Maes IH, Joore MA, Anteunis LJ. Tinnitus interferes with daily life activities: a psychometric examination of the Tinnitus Disability Index. Ear and Hearing 2011;32(5):623-33. [PUBMED: 21336139]

\section{Cima 2011b}

Cima RF, Crombez G, Vlaeuen JW. Catastrophizing and fear of tinnitus predict quality of life in patients with chronic tinnitus. Ear and Hearing 2011;32(5):634-64.

\section{Cima 2014}

Cima RFF, Andersson G, Schmidt CJ, Henry JA. Cognitivebehavioral treatments for tinnitus: a review of the literature. Journal of the American Academy of Audiology 2014;25:29-61.

\section{Cima 2019}

Cima RFF, Mazurek B, Haider H, Kikidis D, Lapira A, Norena A, et al. A multidisciplinary European guideline for tinnitus: diagnostics, assessment, and treatment. HNO 2019;67(Suppl 1):10-42.

\section{Crönlein 2007}

Crönlein T, Langguth B, Geisler P, Hajak G. Tinnitus and insomnia. Progress in Brain Research 2007;166:227-33.

\section{Davis 2000}

Davis A, El Rafaie A. Epidemiology of tinnitus. In: Tyler RS editor(s). Tinnitus Handbook. Singular, Thomson Learning, 2000.

\section{de Ridder 2011}

De Ridder D, Elgoyhen AB, Romo R, Langguth B. Phantom percepts: tinnitus and pain as persisting aversive memory networks. Proceedings of the National Academy of Sciences of the United States of America 2011;108(20):8075-80.

\section{de Ridder 2014}

De Ridder D, Vanneste S, Weisz N, Londero A, Schlee W, Elgoyhen $A B$, et al. An integrative model of auditory phantom perception: tinnitus as a unified percept of interacting separable subnetworks. Neuroscience and Biobehavioral Reviews 2014;44:16-32.

\section{Department of Health 2009}

Department of Health. Provision of Services for Adults with Tinnitus. A Good Practice Guide. London: Central Office of Information, 2009.

\section{Diener 1985}

Diener E, Emmons RA, Larsen RJ, Griffin S. The Satisfaction With Life Scale. Journal of Personality Assessment 1985;49:71-5.

\section{Dietrich 2001}

Dietrich V, Nieschalk M, Stoll W, Rajan R, Pantev C. Cortical reorganization in patients with high frequency cochlear hearing loss. Hearing Research 2001;158:95-101.

\section{Dobie 1999}

Dobie RA. A review of randomized clinical trials in tinnitus. Laryngoscope 1999;109:1202-11.

\section{Dong 2010}

Dong S, Rodger J, Mulders WH, Robertson D. Tonotopic changes in GABA receptor expression in guinea pig inferior colliculus after partial unilateral hearing loss. Brain Research 2010;1342:24-32.

\section{Ecclestone 2013}

Eccleston C, Morley SJ, Williams AC. Psychological approaches to chronic pain management: evidence and challenges. British Journal of Anaesthesia 2013;111(1):59-63.

\section{Eggermont 2004}

Eggermont JJ, Roberts LE. The neuroscience of tinnitus. Trends in Neuroscience 2004;27:676-82.

\section{Eggermont 2014}

Eggermont JJ, Roberts LE. Tinnitus: animal models and findings in humans. Cell and Tissue Research 2014;361(1):311-36.

\section{El-Shunnar 2011}

El-Shunnar S, Hoare DJ, Smith S, Gander PE, Kang S, Fackrell K, et al. Primary care for tinnitus: practice and opinion among GPs in England. Journal of Evaluation in Clinical Practice 2011;17:684-92.

\section{Elgoyhen 2015}

Elgoyhen AB, Langguth B, De Ridder D, Vanneste S. Tinnitus: perspectives from human neuroimaging. Nature Reviews. Neuroscience 2015;16(10):632-42.

\section{Ellis 1977}

Ellis A, Grieger R (editors). Handbook of Rational-Emotive Therapy. Vol. 1, New York: Springer, 1977.

\section{Engineer 2011}

Engineer ND, Riley JR, Seale JD, Vrana WA, Shetake JA, Sudanagunta SP, et al. Reversing pathological neural activity using targeted plasticity. Nature 2011;470:101-4. 


\section{Fackrell 2014}

Fackrell K, Hall DA, Barry J, Hoare DJ. Tools for tinnitus measurement: development and validity of questionnaires to assess handicap and treatment effects. In: Signorelli F, Turjman F editor(s). Tinnitus: Causes, Treatment and Short \& Long-Term Health Effects. New York: Nova Science Publisher, 2014:13-60.

\section{Fowler 1944}

Fowler EP. Head noises in normal and disordered ears: significance, measurement, differentiation and treatment. Archives of Otolaryngology 1944;39:490-503.

\section{Frisch 1992}

Frisch MB, Cornell J, Villanueva M, \& PJ. Clinical validation of the Quality of Life Inventory: a measure of life satisfaction of use in treatment planning and outcome assessment. Psychological Assessment 1992;4:92-101.

\section{Fuller 2017a}

Fuller TE, Haider HF, Kikidis D, Lapira A, Mazurek B, Norena A, et al. Different teams, same conclusions? A systematic review of existing clinical guidelines for the assessment and treatment of tinnitus in adults. Frontiers in Psychology 2017;8:206. [DOI: 10.3389/fpsyg.2017.00206]

\section{Furlong 2001}

Furlong WJ, Feeny DH, Torrance GW, Barr RD. The Health Utilities Index $\left(\mathrm{HUI}^{\circledR}\right)$ system for assessing health-related quality of life in clinical studies. Annals of Medicine 2001;33:375-84.

\section{Gallus 2015}

Gallus S, Lugo A, Garavello W, Bosetti C, Santoro E, Colombo P, et al. Prevalence and determinants of tinnitus in the Italian adult population. Neuroepidemiology 2015;45(1):12-9.

\section{Goebel 1994}

Goebel G, Hiller W. The Tinnitus Questionnaire. A standard instrument for grading the degree of tinnitus. Results of a multicenter study with the tinnitus questionnaire [TinnitusFragebogen (TF). Standardinstrument zur Graduierung des Tinnitusschweregrades. Ergebnisse einer Multicenterstudie mit dem Tinnitus-Fragebogen (TF)]. HNO 1994;42(3):166-72.

\section{Hahn 2008}

Hahn A, Radkova R, Achiemere G, Klement V, Alpini D, Strouhal J. Multimodal therapy for chronic tinnitus. International Tinnitus Journal 2008;14:69-71.

\section{Hall 2011}

Hall D, Láinez M, Newman C, Sanchez T, Egler M, Tennigkeit F, et al. Treatment options for subjective tinnitus: self reports from a sample of general practitioners and ENT physicians within Europe and the USA. BMC Health Services Research 2011;11(1):302.

\section{Hall 2016}

Hall D, Haider H, Szczepek A, Lau P, Rabau S, Jones-Diette J, et al. Systematic review of outcome domains and instruments used in clinical trials of tinnitus treatments in adults. Trials 2016;17(1):270. [DOI: 10.1186/s13063-016-1399-9]

\section{Hall 2018}

Hall DA, Smith H, Hibbert A, Colley V, Haider HF, Horobin A, et al. Core Outcome Measures in Tinnitus (COMiT) initiative. The COMiT'ID Study: developing core outcome domains sets for clinical trials of sound-, psychology-, and pharmacology-based interventions for chronic subjective tinnitus in adults. Trends in Hearing 2018;22:1-16.

\section{Hallam 1984}

Hallam RS, Rachman S, Hinchcliffe R. Psychological aspects of tinnitus. In: Rachman S editor(s). Contributions to Medical Psychology. Oxford: Pergamon Press, 1984.

\section{Hallam 1988}

Hallam RS, Jakes SC, Hinchcliffe R. Cognitive variables in tinnitus annoyance. British Journal of Clinical Psychology 1988;27:213-22.

\section{Hallam 2008}

Hallam RS. Manual of the Tinnitus Questionnaire (TQ). Revised and updated. London: Polpresa Press, 2008.

\section{Hamilton 1960}

Hamilton M. A rating scale for depression. Journal of Neurology, Neurosurgery \& Psychiatry 1960;23(1):56-62.

\section{Han 2009}

Han BI, Lee HW, Kim TY, Lim JS, Shin KS. Tinnitus: characteristics, causes, mechanisms, and treatments. Journal of Clinical Neurology 2009;5:11-9.

\section{Hayes 1999}

Hayes SC, Strosahl KD, Wilson KG. Acceptance and Commitment Therapy: An Experiential Approach to Behavior Change. New York: Guildford Press, 1999.

\section{Hays 1993}

Hays RD, Sherbourne CD, Mazel RM. The RAND 36-item health survey 1.0. Health Economics 1993;2(3):217-27.

\section{Henry 1996a}

Henry JL, Wilson PH. The psychological management of tinnitus: comparison of a combined cognitive educational program, education alone and a waiting-list control. International Tinnitus Journal 1996;2:9-20.

\section{Henry 1998a}

Henry JL, Wilson P. The psychometric properties of two measures of tinnitus complaint and handicap. International Tinnitus Journal 1998;4:114-21.

\section{Henry 2005}

Henry JA, Dennis KC, Schechter MA. General review of tinnitus: prevalence, mechanisms, effects, and management. Journal of Speech, Language, and Hearing Research 2005;48:1204-35.

\section{Hesser 2009}

Hesser H, Westin V, Hayes SC, Andersson G. Clients' in-session acceptance and cognitive defusion behaviors in acceptancebased treatment of tinnitus distress. Behaviour Research and Therapy 2009;47(6):523-8. [PUBMED: 19268281] 


\section{Hesser 2011}

Hesser H, Weise C, Westin VZ, Andersson G. A systematic review and meta-analysis of randomized controlled trials of cognitivebehavioral therapy for tinnitus distress. Clinical Psychology Review 2011;31(4):545-53.

\section{Higgins 2011}

Higgins JPT, Green S (editors). Cochrane Handbook for Systematic Reviews of Interventions Version 5.1.0 [updated March 2011]. The Cochrane Collaboration, 2011. Available from www.cochrane-handbook.org.

\section{Higgins 2013}

Higgins JPT, Lasserson T, Chandler J, Tovey D, Churchill R. Standards for the conduct of new Cochrane Intervention Reviews 2012 V2.3. Methodological Expectations of Cochrane Intervention Reviews: Standards for the conduct and reporting of new Cochrane Intervention Reviews 2012. Version 2. London: Cochrane, 2013.

\section{Higgins 2016}

Higgins JPT, Lasserson T, Chandler J, Tovey D, Churchill R. Methodological Expectations of Cochrane Intervention Reviews. London: Cochrane, 2016.

\section{Hiller 2006}

Hiller W, Goebel G. Factors influencing tinnitus loudness and annoyance. Archives of Otolaryngology--Head and Neck Surgery 2006;132:1323-30.

\section{Hoare 2011a}

Hoare DJ, Hall DA. Clinical guidelines and practice: a commentary on the complexity of tinnitus management. Evaluation and the Health Professions 2011;34(4):413-20.

\section{Hoare 2011b}

Hoare DJ, Kowalkowski V, Kang S, Hall DA. Systematic review and meta-analyses of RCTs examining tinnitus management. Laryngoscope 2011;121:1555-64.

\section{Hoare 2014}

Hoare DJ, Edmondson-Jones M, Sereda M, Akeroyd MA, Hall D. Amplification with hearing aids for patients with tinnitus and co-existing hearing loss. Cochrane Database of Systematic Reviews 2014, Issue 1. [DOI: 10.1002/14651858.CD010151.pub2]

\section{Hobson 2012}

Hobson J, Chisholm E, El Refaie A. Sound therapy (masking) in the management of tinnitus in adults. Cochrane Database of Systematic Reviews 2012, Issue 11. [DOI: 10.1002/14651858.CD006371.pub3]

\section{Hofmann 2008}

Hofmann SG, Asmundson G. Acceptance and mindfulnessbased therapy: new wave or old hat?. Clinical Psychology Review 2008;28(1):1-16.

\section{Holl 2011}

Holl M. Die Tinnitus-Atemtherapie: So gehen Sie aktiv gegen Ihr Ohrgeräusch vor. Hannover: Schluetersche, 2011.

\section{Jacobson 1957}

Jacobson E. You Must Relax. New York: McGraw-Hill, 1957.

\section{Jacobson 1991}

Jacobson NS, Truax P. Clinical significance: a statistical approach to defining meaningful change in psychotherapy research. Journal of Consulting and Clinical Psychology 1991;59(1):12-9.

\section{Jastreboff 1988}

Jastreboff PJ, Brennan JF, Coleman JK, Sasaki CT. Phantom auditory sensation in rats: an animal model for tinnitus. Behavioral Neuroscience 1988;102:811-22.

\section{Jastreboff 1990}

Jastreboff PJ. Phantom auditory perception (tinnitus): mechanisms of generation and perception. Neuroscience Research 1990;8(4):221-54.

\section{Jastreboff 1993}

Jastreboff PJ, Hazell JW. A neurophysiological approach to tinnitus: clinical implications. British Journal of Audiology 1993;27(1):7-17. [PUBMED: 8339063]

\section{Jastreboff 2004}

Jastreboff PJ, Hazell JWP. Tinnitus Retraining Therapy. Cambridge University Press, 2004.

\section{Kabat-Zinn 1982}

Kabat-Zinn J. An outpatient program in behavioral medicine for chronic pain patients based on the practice of mindfulness meditation: Theoretical considerations and preliminary results. General Hospital Psychiatry 1982;4(1):33-47.

\section{Kaldo 2004a}

Kaldo V, Andersson G. Kognitiv Beteendeterapivid Tinnitus [Cognitive-Behavioural Therapy of Tinnitus]. Lund, Sweden: Studentlitteratur, 2004.

\section{Kaplan 1996}

Kaplan R, Ganiats T, Sieber W. The Quality of Well-Being Scale, Self Administered (QWB-SA). San Diego: University of California, San Diego, 1996.

\section{Kim 2015}

Kim HJ, Lee HJ, An SY, Sim S, Park B, Kim SW, et al. Analysis of the prevalence and associated risk factors of tinnitus in adults. PloS One 2015;10(5):e0127578.

\section{Kircher 2008}

Kircher ML, Standring RT, Leonetti JP. Neuroaudiologic assessment of pulsatile tinnitus. Paper presented at the Annual Meeting of the American Academy of Otolaryngology - Head Neck Surgery. Chicago, 2008.

\section{Kleinjung 2016}

Kleinjung T. Pulsatile tinnitus. In: Baguley D, Fagelson M editor(s). Tinnitus. Clinical and Research Perspectives. San Diego: Plural Publishing, 2016:163-80. 


\section{Kleinstauber 2013}

Kleinstauber M, Jasper K, Schweda I, Hiller W, Andersson G, Weise $\mathrm{C}$. The role of fear-avoidance cognitions and behaviors in patients with chronic tinnitus. Cognitive Behaviour Therapy 2013;42(2):84-99. [PUBMED: 23199238]

\section{Kluk 2006}

Kluk K, Moore BCJ. Dead regions in the cochlea and enhancement of frequency discrimination: effects of audiogram slope, unilateral versus bilateral loss, and hearing-aid use. Hearing Research 2006;221:1-15.

\section{Kroenke 2001}

Kroenke K, Spitzer RL, Williams JB. The PHQ-9: validity of a brief depression severity measure. Journal of General Internal Medicine 2001;16(1):606-13.

\section{Kröner-Herwig 1997}

Kröner-Herwig B. Psychologische Behandlung des Chronischen Tinnitus. Stuttgart: Beltz Psychologie Verlags Union, 1997.

\section{Kuk 1990}

Kuk FK, Tyler RS, Russell D, Jordan H. The psychometric properties of a tinnitus handicap questionnaire. Ear and Hearing 1990;11:434-45.

\section{König 2006}

König O, Schaette R, Kempter R, Gross M. Course of hearing loss and occurrence of tinnitus. Hearing Research 2006;221:59-64.

\section{Landry 2019}

Landry EC, Sandoval XCR, Simeone CN, Tidball G, Lea J, Westerberg BD. Systematic review and network meta-analysis of cognitive and/or behavioral therapies (CBT) for tinnitus. Otology \& Neurotology 2019 Nov 15 [Epub ahead of print].

\section{Langers 2012}

Langers DRM, de Kleine E, van Dijk P. Tinnitus does not require macroscopic tonotopic map reorganization. Frontiers in Systems Neuroscience 2012;6:2. [DOI: 10.3389/fnsys.2012.00002]

\section{Langguth 2011}

Langguth B. A review of tinnitus symptoms beyond 'ringing in the ears': a call to action. Current Medical Research and Opinion 2011;27(8):1635-43.

\section{Langguth 2013}

Langguth B, Kreuzer PM, Kleinjung T, De Ridder D. Tinnitus: causes and clinical management. Lancet Neurology 2013;12(9):920-30.

\section{Lethem 1983}

Lethem J, Slade PD, Troup JD, Bentley G. Outline of a fearavoidance model of exaggerated pain perception--I. Behaviour Research and Therapy 1983;21(4):401-8. [PUBMED: 6626110]

\section{Lewinsohn 1978}

Lewinsohn PM, Munoz R, Youngren MA, Zeiss AM. Control Your Depression. New Jersey: Prentice-Hall, 1978.

\section{Lovibond 1995}

Lovibond SH, Lovibond PF. Manual for the Depression Anxiety Stress Scales. Sydney, Australia: Psychology Foundation, 1995.

\section{Maes 2014}

Maes IH, Cima RF, Anteunis LJ, Scheijen DJ, Baguley DM, El Refaie A, et al. Cost-effectiveness of specialized treatment based on cognitive behavioral therapy versus usual care for tinnitus. Otology \& Neurotology 2014;35(5):787-95. [PUBMED: 24829038]

\section{Marciano 2003}

Marciano E, Varrabba L, Giannini P, Sementina C, Verde P, Bruno C, et al. Psychiatric comorbidity in a population of outpatients affected by tinnitus. International Journal of Audiology 2003;42:4-9.

\section{Martines 2010}

Martines F, Bentivegna D, Di Piazza F, Martines E, Sciacca V, Martinciglio G. Investigation of tinnitus patients in Italy: clinical and audiological characteristics. International Journal of Otolaryngology 2010 Jun 23 [Epub ahead of print].

\section{Martinez-Devesa 2010}

Martinez-Devesa P, Perera R, Theodoulou M, Waddell A. Cognitive behavioural therapy for tinnitus. Cochrane Database of Systematic Reviews 2010, Issue 9. [DOI: 10.1002/14651858.CD005233.pub3]

\section{Mazurek 2015}

Mazurek B, Szczepek AJ, Hebert S. Stress and tinnitus. HNO 2015;63(4):258-65.

\section{McDermott 1998}

McDermott HJ, Lech M. Loudness perception and frequency discrimination in subjects with steeply sloping hearing loss: possible correlates of neural plasticity. Journal of the Acoustical Society of America 1998;104:2314-25.

\section{McKenna 2004}

McKenna L. Models of tinnitus suffering and treatment compared and contrasted. Audiological Medicine 2004;2:41-53.

\section{McKenna 2014}

McKenna L, Handscomb L, Hoare DJ, Hall DA. A scientific cognitive-behavioral model of tinnitus: novel conceptualizations of tinnitus distress. Frontiers in Neurology 2014;5:196. [PUBMED: 25339938]

\section{Mehta 2019}

Mehta S, Peynenburg VA, Hadjistavropoulos HD. Internetdelivered cognitive behaviour therapy for chronic health conditions: a systematic review and meta-analysis. Journal of Behavioral Medicine 2019;42:169-87.

\section{Meijerink 2017}

Meijerink JF, Pronk M, Paulissen B, Witte BI, Wouden BV, Jansen V, et al. Effectiveness of an online SUpport PRogramme (SUPR) for older hearing aid users: study protocol for a cluster randomised controlled trial. BMJ Open 2017;7(5):e.015012. 


\section{Meikle 2012}

Meikle MB, Henry JA, Griest SE, Stewart BJ, Abrams HB, McArdle R, et al. The Tinnitus Functional Index: development of a new clinical measure for chronic, intrusive tinnitus. Ear and Hearing 2012;33:153-76.

\section{Michie 2013}

Michie S, Richardson M, Johnston M, Abraham C, Francis J, Hardeman W, et al. The behavior change technique taxonomy (v1) of 93 hierarchically clustered techniques: building an international consensus for the reporting of behavior change interventions. Annals of Behavioral Medicine 2013;46(1):81-95.

\section{Middleton 2011}

Middleton JW, Kiritanid T, Pedersen C, Turner JG, Shepherd GMG, Tzounopoulos T. Mice with behavioral evidence of tinnitus exhibit dorsal cochlear nucleus hyperactivity because of decreased GABAergic inhibition. Proceedings of the National Academy of Science 2011;108:7601-6.

\section{Milerova 2013}

Milerova J, Anders M, Dvorak T, Sand PG, Koniger S, Langguth B. The influence of psychological factors on tinnitus severity. General Hospital Psychiatry 2013;35(4):412-6.

\section{Moher 2009}

Moher D, Liberati A, Tetzlaff J, Altman DG, The PRISMA Group. Preferred Reporting Items for Systematic Reviews and Meta-Analyses: The PRISMA Statement. PLoS Medicine 2009;6(7):e1000097.

\section{Moore 2009}

Moore BCJ, Vinay SN. Enhanced discrimination of lowfrequency sounds for subjects with high-frequency dead regions. Brain 2009;132:524-36.

\section{Mulders 2010}

Mulders WH, Seluakumaran K, Robertson D. Efferent pathways modulate hyperactivity in inferior colliculus. Journal of Neuroscience 2010;30:9578-87.

\section{Munoz 1993}

Munoz R, Miranda J. Group Therapy Manual for Cognitive Behavioral Treatment for Depression. Unpublished, 1993.

\section{Møller 2000}

Møller AR. Similarities between severe tinnitus and chronic pain. Journal of the American Academy of Audiology 2000;11:115-24.

\section{MühInickel 1998}

Mühlnickel W, Elbert T, Taub E, Flor H . Reorganization of auditory cortex in tinnitus (plasticity magnetic source imaging). Proceedings of the National Academy of Sciences of the United States of America 1998;95:10340-3.

\section{Newman 1996}

Newman CW, Jacobson GP, Spitzer JB. Development of the Tinnitus Handicap Inventory. Archives of Otolaryngology--Head and Neck Surgery 1996;122:143-8.

\section{Noreña 2005}

Noreña AJ, Eggermont JJ. Enriched acoustic environment after noise trauma reduces hearing loss and prevents cortical map reorganization. Journal of Neuroscience 2005;25:699-705.

\section{Noreña 2011}

Noreña AJ. An integrative model of tinnitus based on a central gain controlling neural sensitivity. Neuroscience and Biobehavioral Reviews 2011;35:1089-109.

\section{Nyenhuis 2013b}

Nyenhuis N, Golm D, Kroner-Herwig B. A systematic review and meta-analysis on the efficacy of self-help interventions in tinnitus. Cognitive Behaviour Therapy 2013;42(2):159-69.

\section{Ost 1987}

Ost LG. Applied relaxation: description of a coping technique and review of controlled studies. Behaviour Research and Therapy 1987;25(5):397-409. [0005-7967: (Print)]

\section{Pavlov 1927}

Pavlov I. Conditioned Reflexes. London: Oxford University Press, 1927.

\section{Philippot 2012}

Philippot P, Nef F, Clauw L, de Romree M, Segal Z. A randomized controlled trial of mindfulness-based cognitive therapy for treating tinnitus. Clinical Psychology \& Psychotherapy 2012;19(5):411-9. [PUBMED: 21567655]

\section{Phillips 2010}

Phillips JS, McFerran D. Tinnitus Retraining Therapy (TRT) for tinnitus. Cochrane Database of Systematic Reviews 2010, Issue 3. [DOI: 10.1002/14651858.CD007330.pub2]

\section{Pilati 2012}

Pilati N, Large C, Forsythe ID, Hamann M. Acoustic overexposure triggers burst firing in dorsal cochlear nucleus fusiform cells. Hearing Research 2012;283:98-106.

\section{Pitrou 2009}

Pitrou I, Boutron I, Ahmad N, Ravaud P. Reporting of safety results in published reports of randomized controlled trials. Archives of Internal Medicine 2009;169(19):1756-61.

\section{Rademaker 2019}

Rademaker MM, Stegeman I, Ho-Kang-You KE, Stokroos RJ, Smit AL. The effect of mindfulness-based interventions on tinnitus distress. A systematic review. Frontiers in Neurology 2019;10:1135.

\section{Ratnayake 2009}

Ratnayake SA, Jayarajan V, Bartlett J. Could an underlying hearing loss be a significant factor in the handicap caused by tinnitus?. Noise and Health 2009;11:156-60.

\section{Rauschecker 1999}

Rauschecker JP. Auditory cortical plasticity: a comparison with other sensory systems. Trends in Neurosciences 1999;22:74-80. 


\section{Rauschecker 2010}

Rauschecker JP, Leaver AM, Mühlau M. Tuning out the noise: limbic-auditory interaction in tinnitus. Neuron 2010;66:819-26.

\section{Reiss 1986}

Reiss S, Peterson RA, Gursky DM, McNally RJ. Anxiety sensitivity, anxiety frequency and the prediction of fearfulness. Behaviour Research and Therapy 1986;24(1):1-8.

\section{RevMan 2014 [Computer program]}

The Nordic Cochrane Centre, The Cochrane Collaboration. Review Manager (RevMan). Version 5.3. Copenhagen: The Nordic Cochrane Centre, The Cochrane Collaboration, 2014.

\section{Rief 2005}

Rief W, Weise C, Kley N, Martin A. Psychophysiologic treatment of chronic tinnitus: a randomized clinical trial. Psychosomatic Medicine 2005;67:833-8.

\section{Roberts 2010}

Roberts L, Eggermont J, Caspary D, Shore S, Melcher J, Kaltenbach J. Ringing ears: the neuroscience of tinnitus. Journa of Neuroscience 2010;30:14972-9.

\section{Sanchez 2002}

Sanchez TG, Ferrari GMS. The control of tinnitus through hearing aids: suggestions for optimal use [O controle do zumbido por meio de prótese auditiva: sugestões para otimização do uso]. Pró-Fono Revista de Atualização Científica 2002;14:111-8.

\section{Schaette 2011}

Schaette R, McAlpine D. Tinnitus with a normal audiogram: physiological evidence for hidden hearing loss and computational model. Journal of Neuroscience 2011;31:13452-7.

\section{Schecklmann 2013}

Schecklmann M, Lehner A, Poeppl TB, Kreuzer PM, Rupprecht R, Rackl J, et al. Auditory cortex is implicated in tinnitus distress: a voxel-based morphometry study. Brain Structure \& Function 2013;218(4):1061-70.

\section{Schork 2015}

Schork N. Personalized medicine: time for one-person trials. Nature 2015;520:609-11. [DOI: 10.1038/520609]

\section{Schulz 2010}

Schulz KF, Altman DG, Moher D. CONSORT 2010 Statement: updated guidelines for reporting parallel group randomised trials. BMJ 2010;340:c332.

\section{Segal 2012}

Segal ZV, Williams JM, Teasdale JD. Mindfulness-based Cognitive Therapy for Depression. 2nd Edition. New York: Guildford Press, 2012.

\section{Seki 2003}

Seki S, Eggermont JJ. Changes in spontaneous firing rate and neural synchrony in cat primary auditory cortex after localized tone-induced hearing loss. Hearing Research 2003;180:28-38.

\section{Sereda 2018}

Sereda M, Xia J, El Refaie A, Hall DA, Hoare DJ. Sound therapy (using amplification devices and/or sound generators) for tinnitus. Cochrane Database of Systematic Reviews 2018, Issue 12. [DOI: 10.1002/14651858.CD013094.pub2]

\section{Shore 2016}

Shore SE, Roberts LE, Langguth B. Maladaptive plasticity in tinnitus triggers, mechanisms and treatment. Nature Reviews. Neurology 2016;12(3):150-60.

\section{Skevington 2004}

Skevington SM, Lofty M, O'Connell KA. The World Health Organization's WHOQOL-BREF quality of life assessment: psychometric properties and results of the international field trial. A report from the WHOQOL group. Quality of Life Research 2004;13(2):299-310.

\section{Skinner 1938}

Skinner BF. The Behavior of Organisms. New York: AppletonCentury-Crofts, 1938.

\section{Spielberger 1983}

Spielberger C, Gorsuch R, Lushene R, Vagg P, Jacobs, G. Manual for the State-Trait Anxiety Inventory. Palo Alto, CA: Consulting Psychologists Press, 1983.

\section{Spitzer 2006}

Spitzer RL, Kroenke K, Williams JB, Löwe Bl. A brief measure for assessing generalized anxiety disorder: the GAD-7. Archives of Internal Medicine 2006;166(10):1092-7.

\section{Sundell 1982}

Sundell M, Sundell SS. Behavior Modification in the Human Services. Englewood Cliffs, NJ: Prentice-Hall, 1982.

\section{Sweetow 1990}

Sweetow R, Levy M. Tinnitus severity scaling for diagnostic/ therapeutic usage. Hearing Instruments 1990;41:20-1.

\section{Tass 2012}

Tass PA, Adamchic I, Freund H-J, von Stackelberg T, Hauptmann C. Counteracting tinnitus by acoustic coordinated reset neuromodulation. Restorative Neurology and Neuroscience 2012;30:137-59.

\section{Thai-Van 2002}

Thai-Van H, Micheyl C, Noreña A, Collet L. Local improvement in auditory frequency discrimination is associated with hearing loss slope in subjects with cochlear damage. Brain 2002;125:524-37.

\section{Thai-Van 2003}

Thai-Van H, Micheyl C, Moore BCJ, Collet L. Enhanced frequency discrimination near the hearing loss cut-off: a consequence of central auditory plasticity induced by cochlear damage?. Brain 2003;126:2235-45.

\section{Thompson 2016}

Thompson DM, Hall DA, Walker D-M, Hoare DJ. Psychological therapy for people with tinnitus: a scoping review of treatment 
components. Ear and Hearing 2016 Aug 18 [Epub ahead of print].

\section{Tunkel 2014}

Tunkel DE, Bauer CA, Sun GH, Rosenfeld RM, Chandrasekhar SS, Cunningham ER, et al. Clinical Practice Guideline: Tinnitus. Otolaryngology - Head and Neck Surgery 2014;151(2 Suppl):S1-40.

\section{Turk 1983}

Turk DC, Meichenbaum D, Genest M. Pain and Behavioural Medicine - A Cognitive Behavioural Perspective. New York: Guilford Press, 1983.

\section{Van Rillaer 1997}

Van Rillaer J. Learning relaxation in cognitive behavioural therapy [L'apprentissage de la relaxation en thérapie cognitivocomportementale]. Revue Francophone de Clinique Comportementale et Cognitives 1997;2:16-20.

\section{Vanneste 2012}

Vanneste S, De Ridder D. The auditory and non-auditory brain areas involved in tinnitus. An emergent property of multiple parallel overlapping subnetworks. Frontiers in Systems Neuroscience 2012;6:31.

\section{Vlaeyen 2000}

Vlaeyen JW, Linton SJ. Fear-avoidance and its consequences in chronic musculoskeletal pain: a state of the art. Pain 2000;85(3):317-32. [PUBMED: 10781906]

\section{Vlaeyen 2012}

Vlayen JW, Linton SJ. Fear-avoidance model of chronic musculoskeletal pain: 12 years on. Pain 2012;153(6):1144-7. [PUBMED: 22321917]

\section{Wegner 2018}

Wegner I, Hall DA, Smit AL, McFerran D, Stegeman I. Betahistine for tinnitus. Cochrane Database of Systematic Reviews 2018, Issue 12. [DOI: 10.1002/14651858.CD013093.pub2]

\section{Weise 2013}

Weise C, Kleinstäuber M, Hesser H, Westin VZ, Andersson G. Acceptance of tinnitus: validation of the tinnitus acceptance questionnaire. Cognitive Behaviour Therapy 2013;42:100-15.

\section{Weisz 2005}

Weisz N, Moratti S, Meinzer M, Dohrmann K, Elbert T. Tinnitus perception and distress is related to abnormal spontaneous

\section{CHARACTERISTICS OF STUDIES}

Characteristics of included studies [ordered by study ID] brain activity as measured by magnetoencephalography. PLoS Medicine 2005;2:e153.

\section{Wilson 1991}

Wilson PH, Henry J, Bowen M, Haralambous G. Tinnitus Reaction Questionnaire: psychometric properties of a measure of distress associated with tinnitus. Journal of Speech and Hearing Research 1991;34:197-201.

\section{Wilson 1998}

Wilson PH, Henry JL. Tinnitus Cognitions Questionnaire: development and psychometric properties of a measure of dysfunctional cognitions associated with tinnitus. International Tinnitus Journal 1998;4(1):23-30.

\section{Zeman 2011}

Zeman F, Koller M, Figueiredo R, Aazevedo A, Rates M, Coelho C, et al. Tinnitus Handicap Inventory for evaluating treatment effects: which changes are clinically relevant?. Otolaryngology - Head and Neck Surgery 2011;145(2):282-7. [DOI: 10.1177/0194599811403882]

\section{Zigmond 1983}

Zigmond AS, Snaith RP. The Hospital Anxiety and Depression Scale. Acta Psychiatrica Scandinavica 1983;67:361-70.

\section{Zirke 2013a}

Zirke N, Seydel C, Szczepek AJ, Olze H, Haupt H, Mazurek B. Psychological comorbidity in patients with chronic tinnitus: analysis and comparison with chronic pain, asthma or atopic dermatitis patients. Quality of Life Research 2013;22(2):263-72.

\section{Zirke 2013b}

Zirke N, Seydel C, Arsoy D, Klapp BF, Haupt H, Szczepek AJ, et al. Analysis of mental disorders in tinnitus patients performed with Composite International Diagnostic Interview. Quality of Life Research 2013;22(8):2095-104.

\section{References to other published versions of this review Fuller 2017b \\ Fuller TE, Cima RFF, Langguth B, Mazurek B, Waddell A, Hoare DJ, et al. Cognitive behavioural therapy for tinnitus. Cochrane Database of Systematic Reviews 2017, Issue 4. [DOI: 10.1002/14651858.CD012614]}

* Indicates the major publication for the study
Abbott 2009

Methods Two-arm, non-blinded, cluster-RCT, with 6 weeks duration of treatment and pre-post assessment (no follow-up)
Location: Australia 
Setting of recruitment and treatment: participants recruited from 2 major industrial organisations between June 2006 and March 2007 for an online intervention

\section{Sample size: 56}

- Number randomised: 32 (9 work sites) in iCBT, 24 (11 work sites) in information only

- Number completed: 9 in iCBT, 19 in information only

\section{Participant (baseline) characteristics:}

- Age: 50.5 (iCBT), 48.7 (information only)

- Gender: 5 females (1 in iCBT, 4 in information only), 46 males (27 in iCBT, 19 in information only)

- Tinnitus duration: 11.7 years in iCBT, 5.0 years in information only

Inclusion criteria: age between 18 and 65 years, tinnitus for at least 3 months, tinnitus diagnosed by health professional, not currently receiving psychological treatment for tinnitus, and being able to access the Internet and print instructions

Exclusion criteria: none reported

Interventions

іCBT: Internet-based CBT, presented in 6 modules over a period of 6 weeks. Intervention was translated and adapted (shortened) from the Swedish self-help programme used in Kaldo 2008. Modules included: rationale and instructions for applied relaxation; positive imagery to aid relaxation; attentional control exercises; information and advice regarding noise sensitivity; behavioural sleep management strategies; sound enrichment; improving concentration; strategies to make the most of existing hearing abilities; cognitive therapy; future planning; and homework assignments. All modules included the completion and submission of weekly assignments and diaries via email to registered psychologists who provided feedback. The psychologists received supervision via Internet from the Swedish tinnitus team who developed the treatment.

Comparator: information only (IOC). The Tinnitus Information Program contained basic psychoeducational information minus active CBT components, presented over 6 weeks and weekly multiple choice quizzes about participants' memory of the content of the module. The psychologists contacted the IOC participants once a week to provide necessary passwords for each new module and to provide minimal support regarding their tinnitus status and coping. After the 6-week period and the completion of post-assessment measures, the IOC participants were provided the option of completing the intervention programme.

Use of additional interventions: none

Outcomes Primary and secondary outcomes not specified/distinguished

Outcome measures included: Tinnitus Reaction Questionnaire (TRQ), Depression Anxiety and Stress Scale - Depression score (DASS-D), Depression Anxiety and Stress Scale - Anxiety score (DASS-A), Depression Anxiety and Stress Scale - Stress score (DASS-S), World Health Organization Quality of Life Questionnaire Brief version (WHOQOL-BREF); Occupational Stress Inventory revised (OSI-R); visual analogue scales for tinnitus loudness, annoyance, control and sleep quality

Measurement time points: pre and post intervention

Funding sources
Australian Research Council Linkage Project Grant with industry contribution from BP Australia. The role (if any) of the funders was not reported.

\section{Declarations of interest No information provided}

Notes

Participants lost to follow-up: $23 / 32$ (72\%) participants in the intervention group dropped out, 5/24 (21\%) dropped out of comparison group. Intention-to-treat analyses conducted with the baseline scores carried forward and used as post-assessment scores.

Adverse effects: not reported 
Abbott 2009 (Continued)

Risk of bias

\begin{tabular}{|c|c|c|}
\hline Bias & Authors' judgement & Support for judgement \\
\hline $\begin{array}{l}\text { Random sequence genera- } \\
\text { tion (selection bias) }\end{array}$ & Low risk & $\begin{array}{l}\text { Allocation by coin toss and workplace to avoid co-workers being allocated to } \\
\text { different interventions. }\end{array}$ \\
\hline $\begin{array}{l}\text { Allocation concealment } \\
\text { (selection bias) }\end{array}$ & Unclear risk & Information not reported in the manuscript. \\
\hline $\begin{array}{l}\text { Blinding of participants } \\
\text { and personnel (perfor- } \\
\text { mance bias) } \\
\text { All outcomes }\end{array}$ & High risk & $\begin{array}{l}\text { No blinding of participants reported. Quote: "Participants allocated to the In- } \\
\text { formation Only Control condition were informed that they had been random- } \\
\text { ly allocated to first read the online Tinnitus Information Program, after which } \\
\text { they would receive the CBT tinnitus distress program." } \\
\text { Information about personnel blinding was not reported in the manuscript. }\end{array}$ \\
\hline $\begin{array}{l}\text { Blinding of outcome as- } \\
\text { sessment (detection bias) } \\
\text { All outcomes }\end{array}$ & Unclear risk & Information not reported in the manuscript. \\
\hline $\begin{array}{l}\text { Incomplete outcome data } \\
\text { (attrition bias) } \\
\text { All outcomes }\end{array}$ & High risk & $\begin{array}{l}\text { High levels of attrition reported, most of which is unexplained. Missing data } \\
\text { were replaced with the last (observed) outcome carried forward. Quote: "... for } \\
\text { those participants who discontinued their involvement during intervention } \\
(n=20) \text { or control ( } n=7) \text {, their pre-assessment scores were carried forward and } \\
\text { used as the post-assessment scores." }\end{array}$ \\
\hline $\begin{array}{l}\text { Selective reporting (re- } \\
\text { porting bias) }\end{array}$ & High risk & $\begin{array}{l}\text { Not all of the subscales of the WHO QoL and Depression Anxiety and Stress } \\
\text { Scale were included in the repeated measures analyses. }\end{array}$ \\
\hline Other bias & Low risk & No other sources of bias identified. \\
\hline
\end{tabular}

\section{Andersson 2002}

Methods Two-arm, non-blinded, parallel-group RCT, with 6 weeks of treatment and 12 months follow-up

Participants Location: Sweden

Setting of recruitment and treatment: participants were recruited via newspaper articles and the Swedish Hard of Hearing Association website for this online study

\section{Sample size: 117}

- Number randomised: 53 in iCBT, 64 in wait list control

- Number completed: 46 in iCBT, 60 in wait list control

\section{Participant (baseline) characteristics:}

- Age: 48.5 years in iCBT, 47.2 in wait list control

- Gender: $46 \%$ female in iCBT, $48 \%$ female in wait list control

- Tinnitus duration: 6.2 years in iCBT, 6.4 years in wait list control

Inclusion criteria: duration of tinnitus of at least 6 months, having seen a general practitioner (or ear, nose and throat physician) on account of tinnitus, age between 18 and 70 years, and tinnitus a severe problem

Exclusion criteria: not reported 
Andersson 2002 (Continued)

Interventions

iCBT: a CBT self-help manual included 10 components presented in 6 modules on a weekly basis for 6 weeks. The first week included a treatment rationale and the first step of applied relaxation (tense-relax). The second week continued the applied relaxation (relax only) and also included positive imagery, sound enrichment by means of external sounds, hearing tactics and advice regarding noise sensitivity. The latter 2 components were optional. Week 3 involved controlled breathing (as part of applied relaxation) and cognitive therapy, which was adjusted to deal with negative thoughts and beliefs relating to tinnitus. The module given at week 4 included differential relaxation (24) and behavioural sleep management. In the fifth module, rapid relaxation was presented, as was advice regarding concentration difficulties, exercises of concentration (mindfulness) and advice on physical activity. The final module at week 6 included continued practice of applied relaxation, relapse prevention and a summary of the contents of the treatment programme. All modules involved homework assignments and weekly reports on a report web page to be submitted weekly. Participants were encouraged to ask questions regarding the treatment, and all queries were answered as promptly as possible by the investigators depending on their area of expertise. When submitting a week's report, the participant was sent an encouraging email with the instruction to go to the next module.

\section{Comparator: wait list control}

Use of additional interventions: none reported

\section{Outcomes Primary and secondary outcomes were not specified}

The outcome measures included: Tinnitus Reaction Questionnaire (TRQ); Hospital Anxiety and Depression Scale-Anxiety (HADS-A) subscale, Hospital Anxiety and Depression-Depression subscale (HADS-D); Anxiety Sensitivity Index (ASI)

Measurement time points: pre, post and 12 months follow-up

\begin{tabular}{ll}
\hline Funding sources & Swedish Council for Social Research and the Swedish Hard of Hearing Association \\
\hline Declarations of interest & None stated
\end{tabular}

Notes

Participants lost to follow-up: 11 (7 from CBT, 4 from wait list control)

Adverse effects: none (personal communication)

\section{Risk of bias}

\begin{tabular}{|c|c|c|}
\hline Bias & Authors' judgement & Support for judgement \\
\hline \multirow[t]{2}{*}{$\begin{array}{l}\text { Random sequence genera- } \\
\text { tion (selection bias) }\end{array}$} & Unclear risk & $\begin{array}{l}\text { Although the authors state that participants were randomly allocated to } \\
\text { the groups, there is insufficient information about the sequence generation } \\
\text { process available to permit a judgement of low risk or high risk. }\end{array}$ \\
\hline & & $\begin{array}{l}\text { Quote: "Randomization was not stratified and was based on random num- } \\
\text { bers." }\end{array}$ \\
\hline $\begin{array}{l}\text { Allocation concealment } \\
\text { (selection bias) }\end{array}$ & Low risk & $\begin{array}{l}\text { Quote: "Allocation was carried out before any personal E-mail contact with the } \\
\text { participants." }\end{array}$ \\
\hline $\begin{array}{l}\text { Blinding of participants } \\
\text { and personnel (perfor- } \\
\text { mance bias) } \\
\text { All outcomes }\end{array}$ & High risk & $\begin{array}{l}\text { No blinding of personnel or participants and the presence of a wait list control } \\
\text { group. }\end{array}$ \\
\hline $\begin{array}{l}\text { Blinding of outcome as- } \\
\text { sessment (detection bias) } \\
\text { All outcomes }\end{array}$ & Unclear risk & $\begin{array}{l}\text { Although the outcome measures were self-reported/administered question- } \\
\text { naires, there was insufficient information available to permit a judgement of } \\
\text { low risk or high risk. }\end{array}$ \\
\hline
\end{tabular}


Andersson 2002 (Continued)

Incomplete outcome data (attrition bias)

All outcomes
Unclear risk

There were a relatively large number of dropouts, but the reasons for not continuing did not appear to be related to the group allocation.

Quote: "During the first phase of the study, E-mails were sent to the potential dropouts in order to probe for the causes for dropping out or being delayed. A majority of responders replied that the main cause for dropping out or being delayed was lack of time ( $N 22$ ), and 4 participants had just recently begun working with the modules. Additional comments were that the program had been too fast, that there was a lack of peace and quiet at home to do the exercises, and that they had just not been sending in any reports and were still working with the program.A few comments concerned the negative aspects of the program such as being impersonal and that the program was too extensive. Unpaired t tests revealed no significant differences between the completers and the non-responders of the first randomised controlled phase of the study on any of the pretreatment measures (all p values .05). Although the response rate was higher in the WLC group in the first phase, at the 1-year uncontrolled follow-up, the groups did not differ in response rate."

\begin{tabular}{lll}
\hline $\begin{array}{l}\text { Selective reporting (re- } \\
\text { porting bias) }\end{array}$ & Low risk & $\begin{array}{l}\text { No protocol available but all outcomes specified in the manuscript are report- } \\
\text { ed. }\end{array}$ \\
\hline Other bias & Low risk & No other sources of bias identified.
\end{tabular}

\section{Andersson 2005}

Methods

Two-arm, non-blinded, parallel-group RCT, with 6 weeks duration of treatment and 3 months follow-up

Participants

Location: Sweden

Setting of recruitment and treatment: participants were recruited via an advertisement at hospitals and treatment was provided in a psychology department (personal communication)

\section{Sample size: 23}

- Number randomised: 12 to CBT, 11 to wait list control

- Number completed: 12 in CBT, 11 in wait list control

\section{Participant (baseline) characteristics:}

- Age: 70.1 years (overall)

- Gender: 11 females, 12 males

- Tinnitus duration: 13 years

Inclusion criteria: 1 ) aged over 65 years; 2 ) participants should have problems with their tinnitus (for example tinnitus is audible in many acoustic environments, disturbs sleep, or is a dominating problem that affects quality of life); 3) duration of tinnitus for at least 6 months, and be able to come to session$\mathrm{s}$, which included walking the stairs to the therapy room

Exclusion criteria: 1) received previous psychological treatment for tinnitus; 2) had a depression score above 22 on the Beck Depression Inventory; 3) a score above 2 on item 2 (hopelessness) and item 9 (suicidal ideation); or 4) had medical reasons for not taking part in the treatment 6) with 2 MSc students who had completed their training in clinical psychology but were unlicensed at the time (personal communication). CBT aimed to decrease the psychological distress associated with tinnitus and was not targeted towards the loudness of tinnitus. The 6 sessions included: information about tinnitus, applied relaxation (presented during 4 sessions), cognitive restructuring, behavioural activation, positive imagery, sound enrichment (by means of environmental sounds rather than noise 
Andersson 2005 (Continued)

generators), exposure to tinnitus, advice regarding hyperacusis, hearing tactics and relapse prevention. Homework assignments were included in all sessions and comments on assignments were made at the beginning of each session. Additional details of the intervention reported in Andersson 2001.

Comparator: wait list control; participants received CBT after the waiting period

Use of additional interventions: none reported

Outcomes Primary outcome: Tinnitus Reaction Questionnaire (TRQ)

Secondary outcome: Hospital Anxiety and Depression Scale-Anxiety (HADS-A) subscale, Hospital Anxiety and Depression-Depression subscale (HADS-D); Anxiety Sensitivity Index (ASI); daily measures 1 week prior to and after treatment on annoyance, tinnitus loudness and quality of sleep

Measurement time points: pre- and post-treatment and at 3 months follow-up

\begin{tabular}{ll}
\hline Funding sources & This study was supported by a grant from the Swedish Hard of Hearing Association \\
\hline Declarations of interest & Not reported \\
\hline Notes & Participants lost to follow-up: 0 \\
& Adverse effects: none (personal communication) \\
\hline
\end{tabular}

\section{Risk of bias}

\begin{tabular}{|c|c|c|}
\hline Bias & Authors' judgement & Support for judgement \\
\hline $\begin{array}{l}\text { Random sequence genera- } \\
\text { tion (selection bias) }\end{array}$ & Unclear risk & Information not reported in the manuscript. \\
\hline $\begin{array}{l}\text { Allocation concealment } \\
\text { (selection bias) }\end{array}$ & Unclear risk & Information not reported in the manuscript. \\
\hline $\begin{array}{l}\text { Blinding of participants } \\
\text { and personnel (perfor- } \\
\text { mance bias) } \\
\text { All outcomes }\end{array}$ & Unclear risk & Information not reported in the manuscript. \\
\hline $\begin{array}{l}\text { Blinding of outcome as- } \\
\text { sessment (detection bias) } \\
\text { All outcomes }\end{array}$ & Unclear risk & $\begin{array}{l}\text { The outcome measures were self-reported/administered questionnaires, but } \\
\text { there was insufficient information available to permit a judgement of low risk } \\
\text { or high risk. }\end{array}$ \\
\hline $\begin{array}{l}\text { Incomplete outcome data } \\
\text { (attrition bias) } \\
\text { All outcomes }\end{array}$ & Low risk & There was no attrition from the study. \\
\hline $\begin{array}{l}\text { Selective reporting (re- } \\
\text { porting bias) }\end{array}$ & Low risk & $\begin{array}{l}\text { Protocol not available but all outcome measure listed in the methods were re- } \\
\text { ported. }\end{array}$ \\
\hline Other bias & Low risk & No other source of bias identified. \\
\hline
\end{tabular}

\section{Arif 2017}

\begin{tabular}{ll}
\hline Methods & Two-arm, non-blinded, parallel-group RCT, with 15 weeks of treatment and no follow-up \\
\hline Participants & Location: Cardiff, Wales
\end{tabular}


Arif 2017 (Continued)

Setting of recruitment and treatment: the study was conducted between June 2010 and November 2013. Participants were recruited from the tinnitus clinic held at the University Hospital of Wales, Cardiff. Leaflets comprising information about the study were displayed in the University Hospital of Wales and the neighbouring districts' general hospitals. Colleagues at the ENT units from these hospitals were also asked to refer patients to the tinnitus clinic. The treatment itself was conducted at the University Hospital in Cardiff (personal communication).

\section{Sample size: 86}

- Number randomised: 42 in mindfulness meditation group, 44 in relaxation therapy

- Number completed: 34 in mindfulness meditation group, 27 in relaxation therapy

\section{Participant (baseline) characteristics:}

- Age: 53.8 years in mindfulness meditation group, 58.3 in relaxation group

- Gender: $59 \%$ female in the mindfulness meditation group, $48 \%$ female in the relaxation group

- Tinnitus duration: 5.2 years in the mindfulness group, 6.5 years in the relaxation group

Inclusion criteria: patients with a primary complaint of intrusive tinnitus were recruited from the tinnitus clinic held at the University Hospital of Wales, Cardiff. Adults with intrusive tinnitus, aged over 18 years, who had not responded to other treatments such as hearing aids, maskers, background music or reassurance, or patients who did not want to try these treatments, were recruited for the study.

Exclusion criteria: patients with psychiatric disorders severe enough to require treatment were excluded. Those who were undergoing litigation or legal matters related to auditory disorders, those unwilling to consider mindfulness meditation or relaxation therapy, and those who had problems communicating in English were also excluded.

Interventions

Mindfulness: each participant had 5 face-to-face, individual sessions of 40 minutes of mindfulness meditation treatment over a period of 15 weeks. The meditation was conducted by a single experienced therapist.

Mindfulness meditation was used in an attempt to uncouple or neutralise the sensory signal of tinnitus from the interpretative and behavioural response, thereby preventing a weak signal having a powerful conditioned response. The sessions included: exploration of the participant's model of tinnitus; sitting meditation with slow diagphragmatic breathing; addressing avoidance and reassurance seeking behaviours; discussing locus of control; and, homework tasks (e.g. reading material on mindfulness, listening to meditation $\mathrm{CD}$, practising meditation).

Comparator: relaxation group. Each participant had 5 face-to-face, individual sessions of 40 minutes of relaxation over a period of 15 weeks from 1 of 2 therapists who followed a treatment manual based on the work of Lars-Goran Ost (Ost 1987).

The relaxation therapy included: checking understanding of tinnitus; explanation of relaxation response, neural plasticity of the brain; treatment rationale; release only relaxation; visualisation exercises; cue-controlled relaxation; differential relaxation; rapid relaxation; and homework of 15 to 20 minutes relaxation per day.

Use of additional interventions: none reported

Outcomes Primary outcome: Tinnitus Reaction Questionnaire (TRQ)

Secondary outcomes: Hospital Anxiety and Depression Scale-Anxiety (HADS-A) subscale, Hospital Anxiety and Depression-Depression subscale (HADS-D); visual analogue scales to measure the severity and loudness of tinnitus, the sensitivity to loud sounds and the awareness of tinnitus; health state thermometer (a visual analogue scale ranging from 0 to 100 where 0 indicates the worst state of health and 100 the best state of health).

Measurement time points: pre and post intervention

Funding sources No external funding (personal communication) 
Arif 2017 (Continued)

Declarations of interest No conflicts of interest (personal communication)

Notes Participants lost to follow-up: 25/86 (29\%; 8 from the mindfulness group, 17 from the relaxation group)

Adverse effects: none (personal communication)

\section{Risk of bias}

Bias Authors' judgement Support for judgement

Random sequence genera- Unclear risk tion (selection bias)

Although the authors state that participants were randomly allocated to the groups, there is insufficient information about the sequence generation process available to permit a judgement of low risk or high risk.

\begin{tabular}{|c|c|c|}
\hline $\begin{array}{l}\text { Allocation concealment } \\
\text { (selection bias) }\end{array}$ & Low risk & $\begin{array}{l}\text { Quote: "After completing an informed consent form, participants were ran- } \\
\text { domly allocated to one of } 2 \text { groups using sealed envelopes prepared by a per- } \\
\text { son not involved in the trial." (Personal communication). }\end{array}$ \\
\hline
\end{tabular}

Blinding of participants High risk and personnel (perfor-

No blinding described and the outcome was likely to be influenced by lack of mance bias)

All outcomes blinding.

Quote: "Participants who satisfied the above criteria were given written information with brief descriptions of relaxation therapy and mindfulness meditation. The purpose of the research and the potential benefits were also explained. Participants were given the opportunity to ask any questions related to the study at this point. Participants had two weeks to decide whether to participate in the study."

\begin{tabular}{lll}
\hline Blinding of outcome as- & Unclear risk & Although the outcome measures were self-reported/administered question- \\
sessment (detection bias) & naires, there was insufficient information available to permit a judgement of \\
All outcomes & low risk or high risk.
\end{tabular}
All outcomes

Unclear risk

Incomplete outcome data (attrition bias)

There were greater levels of attrition from the relaxation group, but the rea-

All outcomes sons for attrition, with an exception, do not appear to be related (directly) to the effects of the intervention. Quote: "The reasons for not completing the study varied from being too busy to attend, moving out of area, wanting no further treatment, having no time to attend for treatment, not being convinced of treatment benefits and the emergence of other medical problems."

\begin{tabular}{lll}
$\begin{array}{l}\text { Selective reporting (re- } \\
\text { porting bias) }\end{array}$ & Low risk & No protocol available, but all outcomes appear to be reported. \\
\hline Other bias & Low risk & No other sources of bias identified. \\
\hline
\end{tabular}

Beukes 2018a

\begin{tabular}{ll}
\hline Methods & Two-arm, non-blinded, parallel-group RCT, with 8 weeks treatment and 2 months follow-up \\
\hline Participants & Location: England \\
& Setting of recruitment and treatment: recruitment was throughout the United Kingdom for a period \\
of 2 months and targeted people from various demographical backgrounds with significant levels of \\
tinnitus distress. Study information was available online (e.g. the NHS Choices Twitter), in newspapers \\
and magazines (e.g. New Scientist), through support groups (e.g. tinnitus, thyroid) and from healthcare \\
professionals (GP surgeries, audiologists). The study was conducted between October 2016 and the last \\
2-month post-assessment measures were obtained in July 2017. Treatment was conducted online.
\end{tabular}




\section{Sample size: 146}

- Number randomised: 73 in iCBT, 73 in wait list control

- Number completed: 54 in iCBT, 60 in wait list control

\section{Participant (baseline) characteristics:}

- Age: 56.8 years in iCBT, 54.3 years in wait list control, 55.6 overall

- Gender: 63 females (30 in iCBT, 33 in wait list control), 83 males (43 in iCBT, 40 in wait list control)

- Tinnitus duration: 11.1 years in $\mathrm{iCBT}, 12.4$ years in wait list control, 11.7 years overall

Inclusion criteria: 1) aged 18 years and over living in the United Kingdom; 2) computer and Internet access and the ability to use these; 3) the ability to read and type in English; 4) experiencing tinnitus for a minimum duration of 3 months; 5 ) a score of 25 or above on the Tinnitus Functional Index

Exclusion criteria: 1) reporting any major medical, psychiatric, or mental disorder, which may hamper commitment to the programme; 2 ) reporting pulsatile, objective or unilateral tinnitus, which had not been investigated medically; 3 ) tinnitus as a consequence of a medical disorder, still under investigation; 4) undergoing any tinnitus therapy concurrently with partaking in the study

iCBT: Internet-based CBT (iCBT) was based on a previously developed self-help programme for tinnitus (Andersson 2004). The intervention ran over an 8-week period, during which 2 to 3 modules were released on a weekly basis. There were 16 recommended modules and 5 optional modules. Recommended modules included CBT content such as applied relaxation, thought analysis, cognitive restructuring, imagery and exposure techniques. Optional modules were available to add an element of tailoring, and participants could choose whether or not to do these modules.

If initial baseline scores indicated it (criteria specified in the article) specific modules for sleep, concentration and sound sensitivity respectively were recommended to specific participants.

An experienced, registered audiological scientist guided the intervention. The audiologist was experienced in managing tinnitus patients both in a clinical setting and online and had a suitable understanding of CBT principles but no formal CBT training. Supervision was provided by a clinical psychologist (specialised in providing tinnitus intervention) throughout the duration of the trial. The audiologist's role was to conduct the telephone interviews that would last for a minimum of 10 minutes per week. The audiologist would: introduce weekly modules, provide feedback, answer queries, provide guidance, support and encourage engagement.

Comparator: wait list control. Once the experimental group completed the intervention, the wait list control group underwent the same iCBT intervention.

Use of additional interventions: none reported

Outcomes $\quad$ Primary outcome: Tinnitus Functional Index (TFI)

Secondary outcomes: Insomnia Severity Index (ISI), Generalized Anxiety Disorder-7 (GAD-7), Patient Health Questionnaire-9 (PHQ-9), Hearing Handicap Inventory for Adults Screening version (HHIA-S), Hyperacusis Questionnaire (HQ), Cognitive Failures Questionnaires (CFQ), Satisfaction with Life Scales (SWLS); Tinnitus Handicap Inventory Screening version (THI-S) was also administered on a weekly basis

Measurement time points: baseline, post-treatment and 2-month follow-up

Funding sources

This study was not funded from any specific grant from funding agencies in the public, commercial or not-for-profit sectors. Anglia Ruskin, Lamar and Linköping Universities, and NIHR supported the undertaking of this study, but the views expressed are those of the authors and not of these institutions.

Declarations of interest No conflicts of interest are declared

Notes

Participants lost to follow-up: 32 (19 from iCBT, 13 from wait list control)

Adverse effects: no adverse effects were reported during the course of the intervention. However, at 12 months follow-up, 11 out 104 participants indicated that they had experienced: worsening of symptoms, emergence of new symptoms, negative well being and prolongation of treatment. 
Other: intention-to-treat analysis conducted

\section{Risk of bias}

\begin{tabular}{lll}
\hline Bias & Authors' judgement & Support for judgement \\
\hline $\begin{array}{l}\text { Random sequence genera- } \\
\text { tion (selection bias) }\end{array}$ & Low risk & $\begin{array}{l}\text { Randomisation sequence generated by computer algorithm (http://www.ran- } \\
\text { domizer.org/) }\end{array}$ \\
\hline $\begin{array}{l}\text { Allocation concealment } \\
\text { (selection bias) }\end{array}$ & High risk & $\begin{array}{l}\text { Allocation done by an independent researcher but the trial design resulted in } \\
\text { the investigator not being masked to the assignment of interventions during } \\
\text { the running of the trial. }\end{array}$ \\
\hline $\begin{array}{l}\text { Blinding of participants } \\
\text { and personnel (perfor- } \\
\text { mance bias) } \\
\text { All outcomes }\end{array}$ & High risk & $\begin{array}{l}\text { The trial design resulted in the investigator not being masked to the assign- } \\
\text { ment of interventions during the running of the trial. The authors state that } \\
\text { participants might have realised their group assignment, but that this was nev- } \\
\text { er explicitly stated. }\end{array}$
\end{tabular}

Blinding of outcome as- High risk Participants and personnel were not blinded.

sessment (detection bias)

All outcomes

$\begin{array}{ll}\begin{array}{l}\text { Incomplete outcome data } \\ \text { (attrition bias) }\end{array} & \text { Low risk } \\ \text { Alltrition was fully reported and a test for 'missing at random' performed. Mul- } \\ \text { tiple imputation was used in the event of missing data. }\end{array}$

All outcomes

Selective reporting (re- Low risk $\quad$ All outcomes reported as per registered protocol.
porting bias)

Other bias Low risk No other source of bias identified.

Beukes 2018b

Methods Two arm, non-blinded, multi-centre, parallel-group RCT, with 8 weeks duration of treatment and 2-
month follow-up

\section{Participants}

Location: England, 3 sites (Norfolk and Norwich Universities Hospitals Trust, Milton Keynes University Hospital NHS Foundation Trust and Hinchingbrooke Health Care NHS Trust)

Setting of recruitment and treatment: the trial was conducted between October 2016 and July 2017. The 3 sites were selected from sites partnered in the East of England Tinnitus Network to improve consistency of practice across sites. CBT was conducted online and treatment as usual in NHS hospitals and healthcare centres.

\section{Sample size: 92}

- Number randomised: 46 in guided iCBT, 46 in audiological care

- Number completed: 37 in guided iCBT, 37 in audiological care

\section{Participant (baseline) characteristics:}

- Age: 50.7 years in guided iCBT, 55.3 years in audiological care as usual, 53.0 years overall

- Gender: 37 females (17 in guided iCBT, 20 in audiological care as usual), 55 males (29 in guided iCBT, 26 in audiological care)

- Tinnitus duration: 5.2 years in guided iCBT, 7.9 years in audiological care, 6.5 years overall

Inclusion criteria: 1) attending either Norfolk and Norwich University Hospital, Milton Keynes University Hospital or Hinchingbrooke Hospitals as recruitment was via these hospitals; 2) aged 18 years or old- 
er, living in the United Kingdom and having the ability to read and type in English; 3) regular access to a computer and the Internet and the ability to use these; 4) examined clinically by an ear, nose and throat (ENT) specialist and an audiologist to rule out any medical causes for tinnitus; 5) referred to the tinnitus clinic by an ENT specialist or audiologist because of troublesome tinnitus

Exclusion criteria: 1) reporting any major medical, psychiatric or mental disorder which may hamper commitment to the programme; 2 ) undergoing any tinnitus therapy concurrently to partaking in this study

Interventions

iCBT: the content is based on a self-help programme (iCBT) originally developed by Andersson and colleagues (Kaldo 2007). The focus of this intervention is to address the physical, emotional and problematic effects of experiencing tinnitus to aid habituation to tinnitus. Key audiological principles, such as the use of sound enrichment, are also incorporated into the programme. The content of the original programme has been redeveloped for a UK population into an interactive e-learning version. The intervention is partly tailored to individual needs and consists of 16 recommended modules and 5 optional modules. The course features: week 1: programme rationale and outline; week 2: relaxation and imagery; week 3 diaphragmatic breathing, reinterpreting tinnitus, sleep management; week 4: relaxation, focusing and concentration techniques; week 5: relaxation, thought analysis, reducing sound sensitivity; week 6: relaxation in daily activities, cognitive restructuring, communication tactics; week 7: relaxation in stressful situations, exposure to tinnitus; week 8: reviewing helpful techniques, maintenance and relapse prevention.

Participants are instructed to engage with the modules and then practise the suggested techniques on a daily basis. There is a secure messaging system to enable participants to ask questions and allow their assigned audiologists to provide feedback. A synchronous communication with participants about the content of the iCBT was a component of the intervention.

Comparator: audiological care. Participants received individualised therapy for tinnitus using the usual informational counselling approach generally followed in the management of tinnitus in the United Kingdom. This usually consists of 3 appointments of 60 minutes. The initial appointment includes explanations about tinnitus and the effects thereof on the individual's day-to-day life and provides some basic management strategies. A follow-up appointment is made for 1 month later to discuss additional strategies for tinnitus management. One month later a second follow-up appointment may be made to further address remaining difficulties. The appointments may be shorter for those not requiring as much input. The total time under active intervention will be 2 months, on average and follows a structured protocol including similar intervention components in order to standardise the care received across the different hospitals sites.

The content includes: an in-depth case history, information about tinnitus, sound enrichment advice and equipment demonstration, relaxation advice, sleep management advice, CBT techniques such as identifying automatic thoughts, a review of difficulties, advice on further support options and information about further treatment options (e.g. mindfulness).

Use of additional interventions: none reported

Outcomes Primary outcome: Tinnitus Functional Index (TFI) and Tinnitus Handicap Inventory (THI)

Secondary outcomes: Insomnia Severity Index (ISI), Generalized Anxiety Disorder-7 (GAD-7), Patient Health Questionnaire-9 (PHQ-9), Hearing Handicap Inventory for Adults Screening version (HHIA-S), Hyperacusis Questionnaire (HQ), Cognitive Failures Questionnaires (CFQ), Satisfaction with Life Scales (SWLS)

Measurement time points: pre, post and 2 months follow-up

Funding sources The British Society of Audiology funded the study but had no role in the design of the study or in the preparation or approval of the manuscript

\begin{tabular}{ll}
\hline Declarations of interest & No conflicts of interest are declared \\
\hline Notes & Participants lost to follow-up: 18/92 (19.6\%) (9 from iCBT, 9 from treatment as usual) \\
& Adverse effects: none \\
\hline
\end{tabular}


Beukes 2018b (Continued)

Intention-to-treat analyses conducted

\section{Risk of bias}

\section{Bias} tion (selection bias)
Random sequence genera- Low risk

\section{Authors' judgement Support for judgement}

Randomisation sequence was generated by computer algorithm.

Quote (personal communication): "The allocation sequence was set up before the trial started. A computer generated sequence was generated by an independent researcher. This listed the block identifier (up to 20), block size (varying between $4 / 6$ ) and sequence with in the block (1-6). This indicated the list of treatment group $A / B$ to be allocated to (e.g. BAAB, BBAAA, etc). This list was followed to allocate participants. Instead of allocating all the participants at one point in time and letting everyone know their assignment at the same time, patients entered the trial sequentially. First recruited patient would be given Treatment B, second and third recruited participants Treatment A, A, fourth participant Treatment B, and so forth."

$\begin{array}{ll}\begin{array}{l}\text { Allocation concealment } \quad \text { Low risk } \\ \text { (selection bias) }\end{array} & \begin{array}{l}\text { Quote (personal communication): "Allocation concealment was done by pro- } \\ \text { viding the assistant with only study codes. These codes were assigned to a } \\ \text { group. Only following allocation the code was used to identify the patient. The } \\ \text { patient, hospitals and ICBT therapist were then notified of the group assign- } \\ \text { ment and appointments were arranged accordingly." }\end{array} \\ \end{array}$

Blinding of participants High risk Quote: "Whilst a blinded design would be optimal, in this context it is not feasiand personnel (perfor- ble. Both participants and the clinicians will therefore know the group allocamance bias)

All outcomes

\begin{tabular}{lll}
\hline $\begin{array}{l}\text { Blinding of outcome as- } \\
\text { sessment (detection bias) } \\
\text { All outcomes }\end{array}$ & Low risk & The data analysis was masked in terms of group allocation. \\
\hline $\begin{array}{l}\text { Incomplete outcome data } \\
\begin{array}{l}\text { (attrition bias) } \\
\text { All outcomes }\end{array}\end{array}$ & Low risk & No attrition from the study. \\
\hline $\begin{array}{l}\text { Selective reporting (re- } \\
\text { porting bias) }\end{array}$ & Low risk & Protocol and report are consistent. \\
\hline Other bias & Low risk & No other potential biases identified. \\
\hline
\end{tabular}

Cima 2012

Methods Two-arm, non-blinded, parallel-group RCT, with up to 12 weeks of treatment and 4 months follow-up

Participants

Location: the Netherlands

Setting of recruitment and treatment: audiology and communication rehabilitation centre

Sample size: 492

- Number randomised: 245 in CBT, 247 in audiological care

- Number completed: 171 in CBT, 161 in audiological care

Participant (baseline) characteristics: 
- Age: 53.7 years in intervention, 54.6 in comparison, 54.2 years overall

- Gender: 184 females (87 in invention, 97 in comparison), 308 males (158 in intervention, 150 in comparison)

- Tinnitus duration: $30 \%$ less than 1 year, $39 \% 1$ to 5 years; $31 \%$ more than 5 years

Inclusion criteria: adult patients referred to the centre with a primary complaint of subjective tinnitus were eligible for inclusion

Exclusion criteria: patients who were unable to read and write in Dutch, had health issues that impaired attendance or prevented participation (e.g. terminal illness or physical disability), or had undergone treatment at the centre within 5 years before study enrolment. Patients were assessed by an otolaryngologist to rule out pathological changes that needed immediate medical care.

Interventions

CBT: step 1 of specialised care consisted of multidisciplinary diagnostics and specific tinnitus retraining counselling, which were undertaken in a cognitive behaviour framework (including audiological rehabilitation when necessary). When tinnitus was more severe (as measured at baseline and after psychological screening), patients entered step 2 of treatment, which consisted of 3 different 12-week group treatment options with levels of care dependent on tinnitus severity and hearing loss.

Step 2 of specialised care consisted of group treatments (10 to 12 patients, 6 to 8 patients, or 3 to 4 patients, dependent on tinnitus severity level and hearing level) for which the treatment intensity varies according to severity; 120 minutes per session over 12 weeks). The sessions were provided by clinical psychologist, movement therapist, physical therapist, clinical physicist in audiology, social worker and speech therapist. Session content included: psychoeducation, cognitive restructuring, exposure to tinnitus, mindfulness-based elements, stress relief and attention redirecting techniques by means of movement therapy, and applied relaxation.

Themed group information sessions (including partners) were also held. Information sessions were 120 minutes long and provided information about: audiology and tinnitus; thoughts, feelings and behaviours associated with tinnitus; maintaining personal resources/energy and a healthy balance in life; tinnitus and sleep problems; relationships and communication.

An individual trajectory was also provided if group treatment was contraindicated (60 minutes) per discipline per patient for up to 12 weeks.

Comparator group: audiological care. "Treatment as usual in the Netherlands" comprised of 2 steps of care.

Step 1 comprised: audiological diagnostics (105 minutes) with an audiology assistant who conducted pure tone and speech audiometry, tympanometry (stapedial reflexes); tinnitus pitch mask frequency and minimum masking level; uncomfortable loudness level measurement; hearing aid check and optimisation; questions about duration and location of the tinnitus.

It also included an individual consultation with a clinical physicist in audiology, which featured: audiological assessment; an explanation of the results from the assessment; provision of information about tinnitus and hearing loss; an assessment of the severity of tinnitus complaints; prescription of an hearing aid (when indicated by hearing loss).

Audiological rehabilitation (30 minutes) from/with an audiology assistant conducted after 8 weeks of hearing aid use. Audiological follow-up ( 40 minutes) conducted by an audiology assistant included: pre tone and speech audiometry, tympanometry (stapedial reflexes); uncomfortable loudness level measurement; hearing aid check and optimisation (if relevant); tinnitus pitch mask frequency and minimum masking level. Referrals to social work were also made (when indicated).

Step 2 of treatment as usual comprised: intake conducted by a social work (60 minutes) of general inventory of tinnitus complaints and use of hearing aids or maskers, and up to a maximum of 9 follow-up contacts of 60 minutes each when indicated. Follow-up social work contacts could be counselling sessions, telephone contacts, appointments with third parties or home visits.

Use of additional interventions: sound generators were prescribed when specifically asked for by the patient and were adjusted to produce a small band noise around the pitch match frequency and slightly above hearing threshold, as measured with the small band noise of the sound generator. 
Cima 2012 (Continued)

Outcomes

Primary outcome: Health Utilities Index (HUI mark 3), Tinnitus Questionnaire (TQ), Tinnitus Handicap Inventory (THI)

Secondary outcomes: Hospital Anxiety and Depression Scale (HADS) total score, Tinnitus Catastrophizing Scale (TCS), Fear of Tinnitus Questionnaire (FTQ)

Measurement time points: pre, post and 4 months follow-up

Funding sources

The Netherlands Organisation for Health Research and Development (ZonMW) grant number 945-07-715. The sponsor of the study had no role in study design, data collection, data analysis, data interpretation or writing of the report.

Declarations of interest No conflicts of interest were declared.

Notes Participants lost to follow-up: 160 participants (74 in CBT, 86 in audiological care) did not complete assessments at 4 months follow-up

Adverse effects: none

Other: intention-to-treat analyses conducted

\section{Risk of bias}

Bias Authors' judgement Support for judgement

Random sequence genera- Low risk tion (selection bias)

Quote: "An independent research assistant, who was based outside of Adelante, Department of Audiology and Communication, randomly allocated patients by use of a computer-generated allocation sequence to usual care or specialised care in a 1:1 ratio after receipt of informed consent and baseline assessments."

\begin{tabular}{ll}
\hline $\begin{array}{l}\text { Allocation concealment } \\
\text { (selection bias) }\end{array}$ & Low risk \\
& $\begin{array}{l}\text { Quote: "An independent research assistant, who was based outside of Ade- } \\
\text { lante, Department of Audiology and Communication, randomly allocated pa- } \\
\text { tients by use of a computer-generated allocation sequence to usual care or } \\
\text { specialised care in a 1:1 ratio after receipt of informed consent and baseline } \\
\text { assessments." }\end{array}$
\end{tabular}

Blinding of participants Unclear risk Participants and investigators were masked to treatment group allocation. Beand personnel (perfor- fore study enrolment, participants were informed that they would be allocated mance bias)

All outcomes to 1 of 2 different treatments aimed at tinnitus management, with a client-centred, stepped-care approach. Participants were also aware that by giving their consent they would not be informed to which treatment they were allocated. Early in the intervention procedure detailed information about the treatment received was unveiled, while the participants remained masked to the content of the alternative treatment.

\begin{tabular}{|c|c|c|}
\hline $\begin{array}{l}\text { Blinding of outcome as- } \\
\text { sessment (detection bias) } \\
\text { All outcomes }\end{array}$ & Unclear risk & $\begin{array}{l}\text { The outcome measures were self-reported/administered questionnaires, but } \\
\text { there was insufficient information available to permit a judgement of low risk } \\
\text { or high risk }\end{array}$ \\
\hline
\end{tabular}

\begin{tabular}{lll}
\hline $\begin{array}{l}\text { Incomplete outcome data } \\
\text { (attrition bias) } \\
\text { All outcomes }\end{array}$ & Low risk & All available data included in intention-to-treat analyses. \\
\hline $\begin{array}{l}\text { Selective reporting (re- } \\
\text { porting bias) }\end{array}$ & Low risk & All outcomes reported according to the trial registry. \\
\hline Other bias & Low risk & No other sources of bias identified. \\
\hline
\end{tabular}


Davies 1995

Methods

Three-arm, non-blinded, parallel-group RCT, with 1 and 4 months follow-up (treatment duration period not reported)

Participants

Location: London, UK

Setting of recruitment and treatment: potential participants were selected by researchers from outpatients attending a specialist (mainly tertiary referral) neuro-otology clinic. Treatment was conducted in a hospital clinic.

Sample size: 45

- Number randomised: 16 to cognitive therapy, 16 to applied relaxation, 13 to passive relaxation

- Number completed: 10 in cognitive therapy, 11 in applied relaxation, 6 in passive relaxation

Participant (baseline) characteristics (calculated from the participants who completed post-intervention measures):

- Age: 54.5 years in cognitive therapy, 56.9 years in applied relaxation, 58.0 years in passive relaxation; 46.1 years in participants who dropped out

- Gender: 17 females (8 in cognitive therapy, 7 in applied relaxation, 2 in passive relaxation), 13 males ( 3 in cognitive therapy, 5 in applied relaxation, 5 in passive relaxation)

- Tinnitus duration: 4.1 years in cognitive therapy, 5.9 years in applied relaxation, 4.1 years in passive relaxation, 3.2 years in participants who dropped out

Inclusion criteria: 1) tinnitus a significant problem to the client and also the main problem; 2) duration of complaint at least 6 months; 3 ) absence of major psychiatric disorder; 4) able and willing to attend the hospital for therapy; 5) no previous psychological help in the department; 6) able to complete questionnaires without difficulty

Exclusion criteria: not reported

A qualified clinical psychologist, who received additional supervised practice in cognitive therapy prior to the study provided all forms of therapy. All participants were offered a minimum of $6 \times 1$-hour individual sessions with the possibility of extension to a maximum of 8 .

Cognitive therapy: individual cognitive therapy followed the principles of Ellis 1977 and aimed to: 1) render tinnitus "meaningless" and unworthy of attention; and 2) help the participant counter specific thoughts about tinnitus that produce emotional distress. It involved participants identifying any negative thoughts associated with emotional distress, and completing a checklist that listed common tinnitus complaints, associated emotions, and commonly held maladaptive beliefs about tinnitus. Participants also completed an ABC (antecedents, beliefs, consequences) diary and recent incidents formed the basis for cognitive analysis. The participant's evidence for holding maladaptive beliefs about tinnitus (or how to cope with it) were disputed using a Socratic form of questioning and behavioural experiments to test out beliefs were employed where appropriate.

Comparator: passive relaxation group. It was explained that Passive Relaxation Therapy (PRT) would break into the vicious cycle of "annoyance-stress-attention to noises-further annoyance" by diminishing the stress response to tinnitus annoyance. Relaxation was taught in a sitting or lying position in the office and included: a) progressive muscle tensing and relaxing Bernstein 1984; b) use of pleasant visual imagery to promote mental calmness; and c) encouragement of relaxed diaphragmatic breathing.

An audiocassette was supplied and subjects were encouraged to practise at home at least once per day for 20 to 30 minutes. Progress was monitored, problems were dealt with, further practice was given in each session and participants encouraged to apply their relaxation skill in their daily life.

Comparator: applied relaxation group: applied relaxation was taught as for the passive relaxation but in addition it was explained that acquisition of the skill through daily practice would break into the vicious cycle of "annoyance leads to greater attention leads to greater annoyance" by enabling participants to apply relaxation when tinnitus was annoying. Moments of greatest annoyance/distress were 
Davies 1995 (Continued)

recorded in a daily diary in which participants identified antecedents and consequences of these moments.

Participants were instructed to apply the following principles to tinnitus annoyance as a method of coping with it: 1)"When relaxed, focus on the noises and float with them rather than tense up or fight them"; 2) "When relaxed and listening to the noises, search for more pleasant interpretations such as 'wind in trees' rather than 'piercing whistle"; and 3) "When tinnitus is especially distressing, apply relaxation at these times to counteract the learned tendency to tense up to the noise".

Treatment sessions were used to help participants identify situations and record their annoyance and distress so that relaxation could be applied. A hierarchy of situations was constructed and the least distressing was presented once the participant had learned to relax moderately well. Each situation was imagined for 10 to 20 seconds, the participant then took a breath, paused said "relax" (or "calm", "take it easy" etc) and then relaxed the muscles of the body while exhaling. The scene was rehearsed several times before moving up this hierarchy.

Use of additional interventions: all participants received a handout at the end of the screening interview. This included a fact sheet on tinnitus covering medical and audiological aspects, typical psychological effects and the sort of help that could be offered; in addition they received a brief description of the psychological intervention offered to the subject together with its rationale.

\begin{abstract}
Outcomes Primary and secondary outcome measures were not distinguished.
Outcome measures included: Tinnitus Effects Questionnaire (TEQ) short version, tinnitus loudness and annoyance ratings, Beck Depression Inventory (BDI), State Trait Anxiety Inventory (STAI), diary ratings of loudness and annoyance were made for 3 times per day for 7 days prior to the 4-month follow-up assessment; diary insomnia ratings estimating the time it took to get to sleep, whether they had woken during the night and estimate how long they slept. At 4 months follow-up an author blinded to the treatment participants had received conducted a clinical interview to determine the extent that tinnitus was a problem for the participants.
\end{abstract}

Measurement time points: pre, post, 1-month follow-up, and at 4-month follow-up an independent assessor's clinical rating (based on an interview with the participant). The BDI and STAI were administered before treatment and at 1-month follow-up.

Funding sources

The research was supported by the Locally Organised Research Scheme. The role of the funders was not reported.

\begin{tabular}{ll}
\hline Declarations of interest & Not reported \\
\hline Notes & $\begin{array}{l}\text { Participants lost to follow-up: } 6 \text { from cognitive therapy, } 5 \text { from applied relaxation, } 7 \text { from passive re- } \\
\text { laxation }\end{array}$
\end{tabular}

Adverse effects: not reported

Other: applied and passive relaxation data combined to form one group for the purposes of the CBT versus active comparison

Passive relaxation group omitted from the main analyses in the article

\title{
Risk of bias
}

Bias Authors' judgement Support for judgement

Random sequence genera- Unclear risk Information not reported in the manuscript.
tion (selection bias)
tion (selection bias)

Allocation concealment Unclear risk Information not reported in the manuscript.
(selection bias)


Davies 1995 (Continued)

Blinding of participants Unclear risk Information not reported in the manuscript. and personnel (performance bias)

All outcomes

\begin{tabular}{lll}
$\begin{array}{l}\text { Blinding of outcome as- } \\
\text { sessment (detection bias) }\end{array}$ & Unclear risk & $\begin{array}{l}\text { The outcome measures were self-reported/administered questionnaires, but } \\
\text { there was insufficient information available to permit a judgement of low risk } \\
\text { All outcomes }\end{array}$ \\
\hline
\end{tabular}
or high risk.

Incomplete outcome data High risk (attrition bias)

All outcomes
Quote: "More than $50 \%$ of subjects were lost from PRT (see Table 2). This compared with $32-37 \%$ in ART and ICT. Although the proportion lost from Group 1 is not significantly greater than in Groups 2 and 3 combined $(n=45, d f, x 2=1.45$, NS), differential attrition to some extent invalidates the group comparison."

Selective reporting (re- Low risk No protocol available, but all outcomes appear to be reported.
porting bias)

Other bias Low risk No other sources of bias identified.

\section{Henry 1996}

Location: Australia

Setting of recruitment and treatment: participants were primarily referred by audiologists and/ or otolaryngologists at a Veterans Hospital outpatients clinic to the study. Advertisements about the study were also made via radio and the Australian Tinnitus Association. The setting in which the interventions were conducted was not described.

\section{Sample size: 65}

- Number randomised: 22 to cognitive coping skills combined with education, 21 to education only, 22 to wait list control

- Number completed: 16 in cognitive coping skills combined with education, 17 in education only, 14 in wait list control

\section{Participant (baseline) characteristics:}

- Age: 64.6 years overall

- Gender: 8 females and 52 males

- Tinnitus duration: 0 to 5 years: $12 / 60$ participants; 5 to 10 years: $11 / 60$ participants; 10 to 20 years: 19/60 participants; $>20$ years: $18 / 60$ participants

Inclusion criteria: 1) a primary complaint of chronic tinnitus (i.e. "duration greater than six month"), 2) the tinnitus had been assessed by both an otolaryngologist and an audiologist, 3) traditional medical and audiological treatments were not recommended, or had been attempted and had failed, 4) no provision of a hearing aid, masker or tinnitus suppressive medication within the previous 6 months, 5) a demonstrated level of distress associated with tinnitus as indicated by a total score of at least 17 points on the Tinnitus Reaction Questionnaire (TRQ), II 6) able to read and speak English, 7) willing to participate in a research-oriented treatment programme

Exclusion criteria: not reported

Interventions

Cognitive Coping Skills Training plus education: the intervention was delivered in $6 \times 90$-minute, weekly group-based sessions by a clinical psychologist. 
The cognitive techniques employed in this treatment were based largely on the work of Turk 1983 in the area of chronic pain management and Bakal 1972 in the application of psychological interventions for chronic headache. Adaptations were made to the techniques to allow their application to the management of tinnitus.

It was explained to participants that often it is a person's reaction to tinnitus, rather than the sound itself that may lead to distress. Participants were encouraged to learn to approach the problem of tinnitus in more adaptive and constructive ways, and to regard their reaction to tinnitus as potentially manageable and subject to modification.

They were trained in cognitive restructuring and attention diversion strategies (e.g. guided exercises whereby they practised re-focusing attention from internal stimuli to external stimuli; and the use of mental imagery). Participants practised these techniques under guidance from the therapist in the sessions.

Participants were provided with education about tinnitus to ensure thorough knowledge and to resolve any misconceptions. Each participant received a written treatment manual which covered the attention diversion strategies, imagery techniques, thought management skills and the educational material. They were also supplied with audiocassettes of attention diversion and imagery exercises for use in home practice of the techniques.

Comparator 1: Education only comparison. The aim of this treatment programme was solely to educate participants about tinnitus. The material was presented in a written treatment manual. The sessions were didactic in nature and followed a sequence of specific topics each week. Topics covered were: 1 ) the auditory system, language and speech, and the nature of tinnitus; 2 ) audiological assessment; 3) causes of tinnitus; 4) theories of tinnitus and medical treatments; 5) audiological treatments; 6) history of tinnitus, and details of the Australian Tinnitus Association.

Participants of this education-only programme were not instructed in any active coping skills. The educational material was identical to that provided to participants in the combined cognitive coping skills/ education programme. However, the material was provided at a slower pace in the education-only programme.

Comparator 2: wait list control. Participants assigned to the wait list control condition were informed that due to present demands and limited facilities their participation in the programme would be delayed. Participants were assured that they would be treated when further groups were scheduled. Wait list participants received treatment (cognitive coping skills/education) immediately following the posttreatment assessment period.

Use of additional interventions: none reported

\section{Primary and secondary outcome measures were not specified.}

Outcome measures included: Tinnitus Reaction Questionnaire (TRQ); Tinnitus Handicap Questionnaire (THQ); Tinnitus Effects Questionnaire (TEQ); Tinnitus Cognitions Questionnaire (TCQ); Tinnitus Coping Strategies Questionnaire (TCSQ); Tinnitus Knowledge Questionnaire (TKQ); Beck Depression Inventory (BDI); Locus of Control of Behaviour Scale; Self-Monitoring of Tinnitus (participants were asked to monitor for 7 consecutive days how much they noticed their tinnitus, how loud it was and how bothered they were by their tinnitus.

Measurement time points: pre, post, 4, 8, 12 months follow-up

Funding sources Not reported

Declarations of interest Not reported

Notes Participants lost to follow-up: 18/65 (27.7\%; 6 from coping skills with education, 4 from education only, 8 from wait list control)

\section{Adverse effects: not reported}

Other: THQ data used in the analysis. Four- and 8-month follow-up data are not reported in the article. 
Henry 1996 (Continued)

No response to emails requesting additional information about the study.

\section{Risk of bias}

Bias Authors' judgement Support for judgement

Random sequence genera- Unclear risk tion (selection bias)

Although stated in the article that participants were randomly allocated, there is no information on how the randomisation was conducted.

Quote: "The subjects were randomly allocated to one of the three experimental conditions."

\begin{tabular}{lll}
\hline $\begin{array}{l}\text { Allocation concealment } \\
\text { (selection bias) }\end{array}$ & Unclear risk & Information not reported in the manuscript. \\
\hline $\begin{array}{l}\text { Blinding of participants } \\
\text { and personnel (perfor- } \\
\text { mance bias) }\end{array}$ & Unclear risk & Information not reported in the manuscript. \\
All outcomes & \\
\hline
\end{tabular}

\begin{tabular}{|c|c|c|}
\hline $\begin{array}{l}\text { Blinding of outcome as- } \\
\text { sessment (detection bias) } \\
\text { All outcomes }\end{array}$ & Unclear risk & $\begin{array}{l}\text { Quote: "However, it must be acknowledged that these measures are all self-re- } \\
\text { port questionnaires and that the experimenter conducted treatment and as- } \\
\text { sessment sessions." }\end{array}$ \\
\hline
\end{tabular}

Incomplete outcome data Low risk Small amount of attrition and balanced across groups.

(attrition bias)

All outcomes

\begin{tabular}{lll}
\hline $\begin{array}{l}\text { Selective reporting (re- } \\
\text { porting bias) }\end{array}$ & Low risk & $\begin{array}{l}\text { Although no protocol is available, all the outcomes described in method are } \\
\text { reported in the results of the manuscript. }\end{array}$ \\
\hline Other bias & Low risk & No other sources of bias identified. \\
\hline
\end{tabular}

\section{Henry 1998}

\section{Methods}

Participants
Four-arm, non-blinded, parallel-group RCT, with 8 weeks of treatment and 6 months follow-up

Location: Australia

Setting of recruitment and treatment: not reported

\section{Sample size: 50}

- Number randomised: no details provided regarding to which groups participants were allocated

- Number completed: 39

\section{Participant (baseline) characteristics:}

- Age: 56.3 years overall

- Gender: 19 females, 31 males

- Tinnitus duration: not reported

Inclusion criteria: primary complaint of tinnitus of more than 6 months' duration; previous assessment by both an otolaryngologist and an audiologist; traditional medical and audiological treatments not recommended or failed; a demonstrated level of distress associated with tinnitus as indicated by a score of 17 points or higher on the Tinnitus Reaction Questionnaire; ability to speak and read English; willingness to participate in a research-oriented management programme 
Henry 1998 (Continued)

Exclusion criteria: not reported

Interventions

Cognitive restructuring: a rationale for the use of cognitive therapy was provided and that reactions (including avoidance and emotional responses) to tinnitus may be associated with their beliefs and interpretations of the sounds that they experienced.

Participants received instruction in several techniques designed to: 1) identify positive, negative and neutral thoughts and to distinguish between dysfunctional and constructive thinking; 2) manage and control negative thinking (thought stopping); 3) examine the validity of thoughts, challenge negative thoughts, and substitute realistic and rational thoughts (cognitive restructuring); 4) anticipate, prepare for and deal with stressful situations; and 5) employ coping self-statements (self-instructions)

Comparator 1: Attention Control and Imagery Training (ACI). This treatment programme was similar to the one employed in an earlier treatment outcome study (Henry 1996a). The programme involved training in attention control, distraction and imagery skills. Attention control, distraction and imagery skills are cognitive coping strategies which help the participant learn to shift attention to and from the tinnitus and to focus on more pleasant stimuli. The programme was adapted from some of the approaches described for use in the management of chronic pain (Turk 1983) and headache (Bakal 1972).

Attention control involves a series of practice sessions, aided by tape recordings, in which participants attempt to use a number of approaches to divert their attention from the tinnitus to other bodily sensations, images, and external sounds or other stimuli. The aim is not just to learn to divert attention, but to help the person learn that he or she can control the direction of his or her attention.

Participants were also instructed in how to identify positive, negative and neutral thoughts and were introduced to a variety of techniques for controlling negative thoughts, such as thought stopping and increasing positive thoughts (Lewinsohn 1978).

The attention control and imagery exercises were described on audiocassette provided with a detailed manual in order to assist participants in their home practice.

Comparator 2: combined treatment (ACI + CR). Participants in this condition received a combination of the attention control and imagery training plus cognitive restructuring, although the 2 components were necessarily abbreviated in order to ensure that therapy time was equivalent for all 3 treatment groups. Participants were provided with the 2 written treatment manuals and the educational manual.

Comparator 3: wait list control. Participants in this condition were informed that, due to present demands, treatment would be delayed for 8 weeks. Treatment was provided following post-treatment assessment.

Use of additional interventions: all participants were provided with a written educational manual, which gave information about the causes of and treatments for tinnitus.

\section{Outcomes Primary and secondary outcomes not specified/distinguished.}

Outcome measures included: Tinnitus Reaction Questionnaire (TRQ), Tinnitus Handicap Questionnaire (THQ), Tinnitus Effects Questionnaire (TEQ), Tinnitus Cognitions Questionnaire (TCQ), Beck Depression Inventory (BDI), Automatic Thoughts Questionnaire (ATQ)

Measurement time points: pre, post and 6-months follow-up

\begin{tabular}{ll}
\hline Funding sources & Not reported \\
\hline Declarations of interest & Not reported \\
\hline Notes & $\begin{array}{l}\text { Participants lost to follow-up: } 11 \text { in total. Some but not all details were provided regarding the condi- } \\
\text { tions from which participants did not complete the study; } 2 \text { participants who were in the ACl group did } \\
\text { not complete the study, } 2 \text { participants who were in the wait list control did not complete the study and } \\
\text { an additional } 7 \text { did not complete the follow-up assessments. }\end{array}$
\end{tabular}

Adverse effects: not reported 
Henry 1998 (Continued)

Other: no response from authors to questions about the study. Data are not available for meta-analysis.

\section{Risk of bias}

\begin{tabular}{|c|c|c|}
\hline Bias & Authors' judgement & Support for judgement \\
\hline $\begin{array}{l}\text { Random sequence genera- } \\
\text { tion (selection bias) }\end{array}$ & Unclear risk & Information not reported in the manuscript. \\
\hline $\begin{array}{l}\text { Allocation concealment } \\
\text { (selection bias) }\end{array}$ & Unclear risk & Information not reported in the manuscript. \\
\hline $\begin{array}{l}\text { Blinding of participants } \\
\text { and personnel (perfor- } \\
\text { mance bias) } \\
\text { All outcomes }\end{array}$ & Unclear risk & Information not reported in the manuscript. \\
\hline $\begin{array}{l}\text { Blinding of outcome as- } \\
\text { sessment (detection bias) } \\
\text { All outcomes }\end{array}$ & Unclear risk & Insufficient information to judge high or low risk. \\
\hline $\begin{array}{l}\text { Incomplete outcome data } \\
\text { (attrition bias) } \\
\text { All outcomes }\end{array}$ & Unclear risk & $\begin{array}{l}\text { Unclear per condition how many participants under which circumstances } \\
\text { dropped out. }\end{array}$ \\
\hline $\begin{array}{l}\text { Selective reporting (re- } \\
\text { porting bias) }\end{array}$ & Low risk & $\begin{array}{l}\text { No protocol available, but all measures reported in the manuscript are report- } \\
\text { ed for all time points. }\end{array}$ \\
\hline Other bias & Low risk & No other sources of bias identified. \\
\hline
\end{tabular}

Hesser 2012

Methods Three-arm, non-blinded, parallel-group RCT, with 8 weeks treatment and 1 year follow-up

Participants Location: Sweden

Setting of recruitment and treatment: participants were recruited via advertisements in the Swedish media and from a waiting list on the Internet where individuals could report interest in taking part in upcoming Internet-based treatment studies on tinnitus. The treatment was an Internet-based intervention delivered individually to participants.

\section{Sample size: 99}

- Number randomised: 32 to $\mathrm{iCBT}, 35$ to ACT, 32 to online discussion forum

- Number completed: 30 from CBT, 31 from ACT, 32 from online discussion forum

\section{Participant (baseline) characteristics:}

- Age: 48.8 years in iCBT, 50.1 years in ACT, 48.4 in online discussion forum, 48.5 years overall

- Gender: 43 females (14 in iCBT, 15 in ACT, 14 in online discussion forum), 56 males (18 in iCBT, 20 in $A C T, 18$ in online discussion forum)

- Tinnitus duration: 8.9 months in iCBT, 9.7 months in ACT, 9 months in online discussion forum, 9.2 months overall

Inclusion criteria: 1) have had tinnitus for more than 6 months (the diagnosis had to be confirmed by an ear, nose and throat specialist or an audiological physician); 2) to be at least 18 years old; 3 ) to be a resident of Sweden; 4 ) to have moderate to severe tinnitus distress (defined as a total score $\geq 38$ on the 
Tinnitus Handicap Inventory; and 5) given the online format of the treatments participants had to report that they were able to read and write sufficiently well to be able to work with text-based material, had the time to complete the treatment (i.e. a minimum of 2 hours per week for a period of 8 weeks), and had access to a computer with an Internet connection.

Exclusion criteria: 1) have a severe medical or psychiatric condition; 2) presented with an imminent suicide risk; 3) had an ongoing treatment for tinnitus; or 4) previously received the treatments that were offered in the present study.

Interventions

Both treatments were in the form of guided Internet-delivered therapy, which included structured selfhelp material presented via the Internet and an identified therapist (a licensed psychologist or one of 6 clinical psychology masters students) who provided support and guidance of therapeutic activities (Andersson 2009). All online communication with participants was asynchronous (i.e. the therapist and the participant were not concurrently engaged in communication). Treatments consisted primarily of textand picture-based self-help material. The language of instruction and communication was Swedish.

Participants were given one module at a time and were prompted to work approximately 1 week per module during the 8 weeks of treatment.

iCBT: The CBT self-help material (157 pages of text divided into 8 modules) was based on a shortened version of a published self-help manual (Kaldo 2004a). Tinnitus-specific CBT techniques included applied relaxation, positive imagery, attention training, cognitive restructuring, exposure and the use of background sounds to cope with the experience of tinnitus. In addition, throughout the programme participants were provided with the opportunity to work with specific problems that are commonly experienced by individuals with tinnitus, including noise sensitivity, hearing problems and sleep problems, using traditional cognitive and behavioural interventions (e.g. sleep restriction, problem solving, hearing tactics). Interventions that targeted these specific problem areas were optional, and participants were advised to use them if they experienced such problems.

iACT: this ACT self-help treatment manual (104 pages divided into 8 modules) was informed by Hayes' model of psychological flexibility (Hayes 1999). Specific ACT interventions included exercises that focused on mindfulness and distancing of internal experiences (i.e. diffusion), assignments with the purpose of identifying personal values and goals, and exercises that promoted willingness to experience tinnitus in the context of value-based behaviour change. ACT included mindfulness exercises that were not provided to participants in CBT.

All participants in ACT were also offered behavioural interventions that were optional and that targeted specific tinnitus-related problems (i.e. hearing, noise sensitivity and sleep problems). However, all optional interventions were modified to fit with underlying principles of the ACT model. In other words, all control-based parts of the interventions were removed and replaced with ACT-coherent interventions. For example, distraction or cognitive restructuring was replaced with mindfulness or defusion exercises in ACT.

Comparator: online discussion forum. Participants assigned to the control condition were invited to participate in a confidential moderated online discussion forum that specifically targeted tinnitus-related problems. They were encouraged to actively take part in the discussion by posting messages online. Therapists monitored the forum and each week a therapist posted a new topic for the participants to discuss. Participants could read the discussions without being active.

Use of additional interventions: none reported

Outcomes

Primary outcome: Tinnitus Handicap Inventory (THI)

Secondary outcomes: Hospital Anxiety and Depression Scale-Anxiety (HADS-A) subscale, Hospital Anxiety and Depression-Depression subscale (HADS-D); Tinnitus Acceptance Questionnaire (TAQ); Insomnia Severity Index (ISI); the Quality of Life Inventory (QoLI)

Measurement time points: baseline data collected 2 weeks before treatment began, post-treatment data collected at 10 weeks after baseline and 1 year follow-up

Funding sources Swedish Council for Working Life and Social Research and the Swedish Research Council (HEAD Linneaus grant) 
Hesser 2012 (Continued)

Declarations of interest Not reported

Notes

Participants lost to follow-up: 6 out of 99 participants did not complete 1-year follow-up (2 were from CBT group, 4 were from ACT)

Adverse effects: not reported

Other: $\mathrm{ACT}$ and CBT groups combined for the purpose of the comparison CBT versus other experimen-

tal control.

Intention-to-treat analyses conducted.

\title{
Risk of bias
}

\begin{tabular}{lll}
\hline Bias & Authors' judgement & Support for judgement \\
\hline $\begin{array}{l}\text { Random sequence genera- } \\
\text { tion (selection bias) }\end{array}$ & Low risk & $\begin{array}{l}\text { Quote: "A researcher who was blind to pre-assessment data conducted } \\
\text { the randomisation by using an online true random-number service http:// } \\
\text { www.random.org) to generate a randomisation list with no restriction and in- } \\
\text { dependently assigned participants to the conditions and therapists to partici- } \\
\text { pants." }\end{array}$ \\
\hline $\begin{array}{l}\text { Allocation concealment } \\
\text { (selection bias) }\end{array}$ & Low risk & $\begin{array}{l}\text { Quote: "A researcher who was blind to pre-assessment data conducted } \\
\text { the randomisation by using an online true random-number service http:// } \\
\text { www.random.org) to generate a randomisation list with no restriction and in- } \\
\text { dependently assigned participants to the conditions and therapists to partici- } \\
\text { pants." }\end{array}$
\end{tabular}

\begin{tabular}{|c|c|c|}
\hline $\begin{array}{l}\text { Blinding of participants } \\
\text { and personnel (perfor- } \\
\text { mance bias) } \\
\text { All outcomes }\end{array}$ & High risk & No blinding of participants or personnel. \\
\hline $\begin{array}{l}\text { Blinding of outcome as- } \\
\text { sessment (detection bias) } \\
\text { All outcomes }\end{array}$ & Unclear risk & $\begin{array}{l}\text { Although the outcome measures were self-reported/administered question- } \\
\text { naires, there was insufficient information available to permit a judgement of } \\
\text { low risk or high risk. }\end{array}$ \\
\hline $\begin{array}{l}\text { Incomplete outcome data } \\
\text { (attrition bias) } \\
\text { All outcomes }\end{array}$ & Low risk & $\begin{array}{l}\text { Low attrition rates with details provided that include a comparison between } \\
\text { those who had missing with those who completed the study. The comparison } \\
\text { indicated that clinical and demographic details were not related to missing da- } \\
\text { ta. }\end{array}$ \\
\hline $\begin{array}{l}\text { Selective reporting (re- } \\
\text { porting bias) }\end{array}$ & Low risk & $\begin{array}{l}\text { No protocol available, but no reason to suspect that outcomes were not fully } \\
\text { reported. }\end{array}$ \\
\hline Other bias & Low risk & No other sources of bias identified. \\
\hline
\end{tabular}

Jakes 1986

Methods Two-arm, non-blinded, parallel-group RCT, with 5 weeks duration of treatment and 4 months follow-up

Participants

\begin{abstract}
Location: England
Setting of recruitment and treatment: the patients were selected from consecutive attenders at a hospital neuro-otology clinic which accepts secondary and tertiary referrals. Each patient had recently received a medical examination and, in some cases, treatment of the tinnitus setting of treatment not reported.
\end{abstract}


Jakes 1986 (Continued)

\section{Sample size: 24}

- Number randomised: not reported how many participants were in the intervention and comparison groups

- Number completed: 24

Participant (baseline) characteristics:

- Age: 55 years overall

- Gender: 12 females, 12 males

- Tinnitus duration: not reported

Inclusion criteria: not reported

Exclusion criteria: 1) aged 70 years or over; 2 ) people who had recently been provided with a hearing aid, masker or tinnitus-suppressive drugs; or 3) there was a treatable cause of the tinnitus

Interventions

Progressive muscle relaxation, plus attention switching: progressive muscle relaxation plus practice in 'attention-switching' (PMRAS). In the PMRAS group, relaxation training was slightly briefer than the progressive muscle relaxation comparison intervention. When the patients were relaxed, they were instructed to focus alternately (for 20 to 30 sets) on their own tinnitus noises and then on distracting stimuli. According to preference, these were external background sounds or pleasant mental images. The training period was approximately 10 minutes/session. The patients were encouraged to use attention switching at the times when they were most aware of the noises, as well as during their own relaxation practice. During the therapy phase, the experimenters avoided discussion of topics unrelated to tinnitus, and their advice concerning tinnitus was limited to reiterating points contained in the orientation booklet. The patients were reassessed at the beginning of the fifth session. This was followed by a training exercise and then they were instructed to continue to practise daily for 4 weeks before being seen again.

Comparator: progressive muscle relaxation. Progressive muscle relaxation was conducted individually in 5 weekly training sessions each of which lasted 30 minutes. The method of Bernstein 1973 was employed. The patients progressed from lying on a couch to relaxing sitting up in a chair, and were instructed to practise at least once daily with a cassette provided for them, and to attempt to become aware of their state of tension in a variety of situations. They were advised to use relaxation as a general 'stress' reducer, and to facilitate sleep (if the latter was a problem), but they were not given any systematic instructions to use tinnitus or the distress which it occasions, as a cue for relaxation. Suggestions of calmness and relaxing imagery were used during training, but there was no attempt to incorporate tinnitus sensations into the imagery.

Use of additional interventions: none reported

Outcomes Primary and secondary outcomes were not specified.

Outcome measures included: a diary where patients recorded perceived loudness and annoyance respectively in the morning, afternoon and evening; Tinnitus Effects Questionnaire subscales distress, intrusiveness and sleep respectively (TEQ); Interference with Daily Activities (IWDA); Hamilton Rating Scale for Depression (HRD); and sleep using items on the HRD to measure insomnia; Crown-Crisp Experiential Index (CCEI)

Measurement time points: baseline, 1 and 4 months follow-up

\begin{tabular}{ll}
\hline Funding sources & Not reported \\
\hline Declarations of interest & Not reported \\
\hline Notes & $\begin{array}{l}\text { Participants lost to follow-up: unclear - possibly 4 patients were lost to follow-up/excluded but re- } \\
\text { placed over the course of the trial }\end{array}$
\end{tabular}

Adverse effects: not reported 
Other: this study is not included in meta-analysis due to outcome data for all participants being combined for the respective outcome measures

\section{Risk of bias}

\begin{tabular}{|c|c|c|}
\hline Bias & Authors' judgement & Support for judgement \\
\hline $\begin{array}{l}\text { Random sequence genera- } \\
\text { tion (selection bias) }\end{array}$ & Unclear risk & Information not reported in the manuscript. \\
\hline $\begin{array}{l}\text { Allocation concealment } \\
\text { (selection bias) }\end{array}$ & Unclear risk & Information not reported in the manuscript. \\
\hline $\begin{array}{l}\text { Blinding of participants } \\
\text { and personnel (perfor- } \\
\text { mance bias) } \\
\text { All outcomes }\end{array}$ & Unclear risk & Information not reported in the manuscript. \\
\hline $\begin{array}{l}\text { Blinding of outcome as- } \\
\text { sessment (detection bias) } \\
\text { All outcomes }\end{array}$ & Unclear risk & Information not reported in the manuscript. \\
\hline $\begin{array}{l}\text { Incomplete outcome data } \\
\text { (attrition bias) } \\
\text { All outcomes }\end{array}$ & Unclear risk & Information not reported in the manuscript. \\
\hline $\begin{array}{l}\text { Selective reporting (re- } \\
\text { porting bias) }\end{array}$ & High risk & Primary outcome not specified. \\
\hline Other bias & Low risk & No other sources of bias identified. \\
\hline
\end{tabular}

Jakes 1992

Methods Five-arm, non-blinded, parallel-group RCT, with 5 weeks treatment and 3 months follow-up

Participants Location: London, England

Setting of recruitment and treatment: participants were recruited from outpatients attending a specialist neuro-otology clinic. The location of therapy was not specifically reported, although it seems likely to have been conducted at the clinic from which the participants were recruited from.

\section{Sample size: 84}

- Number randomised: 12 in Group Cognitive Therapy (GCT) intervention, 12 in masking comparison, 14 in GCT + masking comparison, 14 in placebo masking comparison, 14 in wait list control comparison

- Number completed: it is not reported how many in Group Cognitive Therapy (GCT) intervention completed the 12- to 24-month follow-up assessment (although 8 participants completed the post-treatment assessment and 9 completed the 3-month follow-up), 10 in masking comparison, 11 in GCT + masking comparison, 12 in placebo masking comparison, 9 in wait list control comparison

\section{Participant (baseline) characteristics:}

- Age: 59.2 years in GCT, 58.2 years in masking, 59.7 years in GCT + masking, 69.3 years in placebo masking, 54.2 years in wait list control

- Gender: 44 females (18 in GCT, 4 in masking, 6 in GCT + masking, 8 in placebo masking, 8 in wait list control); 40 males (12 males in GCT, 8 in masking, 8 in GCT + masking, 6 in placebo masking, 6 in wait list control) 
- Tinnitus duration: 33 participants had duration of tinnitus for less than 2 years, 36 participants had tinnitus for 3 to 10 years, 15 participants had tinnitus for more than 10 years

Inclusion criteria: 1 ) able to hear in a group; 2 ) tinnitus a significant problem to the client; 3 ) duration of complaint at least 1 year; 4) tinnitus the most significant problem for the client; 5) no major psychiatric disorder; 6) able and willing to attend the hospital for treatment; 7) no previous treatment with maskers or cognitive therapy; (8) able to complete questionnaires without difficulty

Exclusion criteria: major psychiatric disorder (usually severe clinical depression)

Interventions

Group cognitive therapy (GCT): 5 sessions of group cognitive therapy (groups of 6 ) were given by 2 therapists who were experienced cognitive/behavioural therapists but had not received extended training in cognitive therapy. All participants were given a booklet (after the initial baseline assessment) explaining the importance of attitudes towards tinnitus in maintaining attention to it and how these could be changed by cognitive therapy.

The content and schedule for the 5 weeks was: week 1 , introductions and explanations of how their tinnitus affected them, the cognitive model of emotional distress was presented and discussed; weeks 2 to 5 involved training clients in recognition, detection and challenging of negative automatic thoughts.

Comparator: masking. A standard masker was fitted monaurally in a quiet room. The masker generated wide-band noise which can be adjusted by high- or low-frequency clipping. Tone adjustments were made and the volume of the masker was adjusted in an attempt to achieve masking of the tinnitus. If masking was not attainable, then the tone controls were adjusted to yield the most comfortable sound possible. Participants were shown how to put the masker on and how to adjust the volume control. They were asked to use the masker for a trial period of 5 weeks. Participants were encouraged to use the masker regularly and also to use it when the tinnitus was distressing. They were told to turn the masker up so that they could not hear the tinnitus. If this was not possible they were advised to set the masker at a comfortable level of loudness.

Comparator: GCT + masking. Group Cognitive Therapy Plus Masker (GCT + AM) combined the GCT, described above, with the concurrent fitting of a masker. The masker was fitted individually prior to the commencement of the group. Discussion of the masker was discouraged in the group.

Comparator: placebo masking. The procedure in this condition was essentially the same as in masker therapy except that the "masker" which was provided emitted a very quiet noise; i.e., during the fitting the noise emitted by the masker was adjusted so that it was at the participants' threshold. The volume control was then glued in place so that the volume could not be increased. Participants were told that although the device they were being given emitted a very quiet sound, this sort of device had been found very helpful by some tinnitus sufferers.

Comparator: wait list control. Participants were told that there was a waiting list and that treatment could not be given for 7 weeks. At the end of this period they were offered treatment, which in most cases consisted of relaxation training or the provision of a masker.

Use of additional interventions: none

\section{Outcomes Primary and secondary outcomes were not specified.}

Outcome measures included: Tinnitus Effects Questionnaire (TEQ), Interference With Daily Activities (IWDA), Crown Crisp Experiential Index (CCEI), use of diary of loudness and tinnitus annoyance

Measurement time points: pre, post and 3 months follow-up

\begin{tabular}{ll}
\hline Funding sources & Locally Organised Research Scheme \\
\hline Declarations of interest & Not reported \\
\hline
\end{tabular}

Notes

Participants lost to follow-up: data were not collected from 19 participants out of $52(37 \%)$ at 3 months follow-up (wait list control not included). Data were missing for $3 / 12$ participants from GCT, 5/12 from masking, 5/14 GCT+ masking, 6/14 from placebo masking. 
Jakes 1992 (Continued)

Adverse effects: not reported

Other: after each group had been randomly allocated 8 to 10 participants, subsequent participants were allocated to an additional GCT group $(n=18)$, which was not included in the analysis of outcome data. However, all participants allocated to GCT were included in the descriptive information.

\section{Risk of bias}

\begin{tabular}{|c|c|c|}
\hline Bias & Authors' judgement & Support for judgement \\
\hline $\begin{array}{l}\text { Random sequence genera- } \\
\text { tion (selection bias) }\end{array}$ & Unclear risk & Information not reported in the manuscript. \\
\hline $\begin{array}{l}\text { Allocation concealment } \\
\text { (selection bias) }\end{array}$ & Unclear risk & Information not reported in the manuscript. \\
\hline $\begin{array}{l}\text { Blinding of participants } \\
\text { and personnel (perfor- } \\
\text { mance bias) } \\
\text { All outcomes }\end{array}$ & Unclear risk & Information not reported in the manuscript. \\
\hline $\begin{array}{l}\text { Blinding of outcome as- } \\
\text { sessment (detection bias) } \\
\text { All outcomes }\end{array}$ & Unclear risk & $\begin{array}{l}\text { In nearly all cases the experimenter remained blind to the type of help re- } \\
\text { ceived until the last question, which usually elicited revealing comments } \\
\text { about the group allocation. }\end{array}$ \\
\hline $\begin{array}{l}\text { Incomplete outcome data } \\
\text { (attrition bias) } \\
\text { All outcomes }\end{array}$ & Unclear risk & Small amount of missing data, but it is not clear how it was managed. \\
\hline $\begin{array}{l}\text { Selective reporting (re- } \\
\text { porting bias) }\end{array}$ & High risk & $\begin{array}{l}\text { Diary rating of loudness and annoyance not reported but this would not be in } \\
\text { cluded in the meta-analysis. }\end{array}$ \\
\hline Other bias & Low risk & No other sources of bias identified. \\
\hline
\end{tabular}

Jasper 2014

$\begin{array}{ll}\text { Methods } & \begin{array}{l}\text { Three-arm, non-blinded, parallel-group RCT, with 10-week duration of treatment and 6-month fol- } \\ \text { low-up }\end{array}\end{array}$

Participants

\section{Location: Germany}

Setting of recruitment and treatment: participants were recruited via a waiting list for tinnitus treatment at the University Outpatient Clinic for Psychotherapy in Mainz, as well as via the German Tinnitus Association, tinnitus self-help groups, pharmacies, private practices, ear, nose and throat practitioners, and the public media. Treatment was conducted online for 2 of the groups (iCBT and Internet-based discussion forum). The setting of the Group CBT intervention was not reported.

\section{Sample size: 128}

- Number randomised: 41 in iCBT, 43 Group CBT, 44 in Internet-based discussion forum

- Number completed: 34 in iCBT, 37 Group CBT, 43 in Internet-based discussion forum

\section{Participant (baseline) characteristics:}

- Age: 51.3 (iCBT), 50.2 (Group CBT), 52.1 (comparison)

- Gender: 51 females (16/41 iCBT, 19/43 Group CBT, 16/44 comparison), 77 males (25/41 iCBT, 24/43 Group CBT, 28 discussion forum)

- Tinnitus duration: 9.2 years in $\mathrm{ICBT}, 8.4$ years in Group CBT, 8.0 in discussion forum group 
Inclusion criteria: criteria for study inclusion were: 1 ) age of $\geq 18$ years; 2 ) a score of $\geq 18$ on the Tinnitus Handicap Inventory (THI) or a score of $\geq 8$ on the Mini-Tinnitus Questionnaire (Mini-TQ); 3) a tinnitus duration of $\geq 6$ months; 4 ) tinnitus as the primary problem (not e.g. as a consequence of morbus Ménière); 5) consenting to be randomised; 6) Internet access; 7) willingness and ability to attend the weekly group sessions; 8 ) no anticipated absence of $>2$ weeks during the course of the study; 9) no CBT for tinnitus within the last 2 years; 10) no ongoing psychological tinnitus treatment; 11) no major medical or psychiatric condition, and 12) no acute suicidality

Exclusion criteria: not reported

Interventions

iCBT: weekly online individual intervention facilitated by Masters level clinical psychologists. German version adapted from the Swedish treatment manual developed by Kaldo 2004a. The iCBT consisted of 12 mandatory (applied relaxation, positive imagery, focus exercises (enhancing the ability to shift the focus from tinnitus to other stimuli, exposure to tinnitus, cognitive restructuring, avoidance behaviour) and 6 optional (sound enrichment, tinnitus re-framing, noise sensitivity, sleep management, concentration management, hearing tactics) text modules. Each module included general information, suggestions for exercising, worksheets and solutions for common problems.

Group CBT: weekly 90-minute group-based sessions provided by clinical psychologists. The group sizes varied from 5 to 12 participants. The specific content included: warming up and general information, progressive muscle relaxation, psychoeducation (information about epidemiology, causes and mechanism of tinnitus distress), cognitive restructuring, focus exercises (enhancing the ability to shift the focus from tinnitus to other stimuli), identification and reduction of avoidance behaviours, patient-doctor communication, relapse prevention, optional topics (positive imagery, stress management, sleep management, hearing tactics). Sharing experiences, discussing individual coping strategies, and demonstrating exercises were important components of the treatment. To facilitate understanding and practice, the participants were given handouts and encouraged to complete homework assignments.

Comparator: Internet-based discussion forum. In order to control for non-specific effects such as increased attention or empathy, a Internet-based discussion forum (DF) was included as a control condition. In the DF, a new discussion topic was presented every week. [Note: the specific topics for each week were not reported.] The participants were encouraged to discuss and to comment on each other's postings. The topics did not include any strategies to improve tinnitus distress but instead focused on individual experiences and attitudes concerning tinnitus (e.g. "Do you think that the topic of "tinnitus" is over- or underrepresented in the public media?"). The forum was closely monitored to make sure postings were appropriate.

Use of additional interventions: none

Outcomes

Primary outcomes: Tinnitus Handicap Inventory (THI) and Mini-TQ

Secondary outcomes: Hospital Anxiety and Depression Scale-Anxiety (HADS-A) subscale, Hospital Anxiety and Depression-Depression subscale (HADS-D), Tinnitus Acceptance Questionnaire (TAQ), Insomnia Severity Index (ISI)

Measurement time points: pre, post and 6-months follow-up

\begin{tabular}{ll}
\hline Funding sources & Not reported \\
\hline Declarations of interest & Not reported \\
\hline
\end{tabular}

Notes

\section{Participants lost to follow-up: 14}

Adverse effects: not reported

Other: data from $\mathrm{iCBT}$ and group CBT combined for the comparison, CBT versus other active experimental control

\section{Risk of bias}

Bias Authors' judgement Support for judgement


Jasper 2014 (Continued)

Random sequence genera- Low risk tion (selection bias)
Quote: "Randomization was achieved by an online service which uses a pseudo-random number algorithm (www.randomization.com) and was conducted by an independent psychologist."

$\begin{array}{ll}\begin{array}{l}\text { Allocation concealment } \\ \text { (selection bias) }\end{array} & \text { Low risk }\end{array}$

Unclear risk

Blinding of participants and personnel (perfor-

No blinding of participants or personnel, but it is unclear whether the differ-

mance bias)

All outcomes ences in treatment credibility/expectancy would have affected performance.

Quote: "Prior to randomisation, the participants rated their preferred treatment as well as the treatment credibility of each intervention. Of all patients, $46 \%$ preferred GCBT and $21 \%$ were in favour of Internet-based treatment; the remaining $33 \%$ stated no preference. No significant differences between the 2 treatment groups were observed regarding their treatment preference $[\times 2(2$, $n=128)=0.54, p=0.762]$. According to the Credibility/Expectancy Questionnaire, treatment credibility/expectancy was rated significantly higher for GCBT (mean $=36.43, \mathrm{SD}=7.97)$ than for ICBT $[$ mean $=33.16, \mathrm{SD}=9.24 ; \mathrm{t}(126)=-4.01$, $\mathrm{p}<0.001]$."

Blinding of outcome as- Low risk sessment (detection bias) All outcomes
Although there was no blinding, the analysis of treatment expectations and credibility showed no relationship with outcomes.

\begin{tabular}{lll}
\hline $\begin{array}{l}\text { Incomplete outcome data } \\
\text { (attrition bias) } \\
\text { All outcomes }\end{array}$ & Low risk & $\begin{array}{l}\text { Similar levels of missing data across groups and multiple imputation and com- } \\
\text { plete case analyses used. }\end{array}$ \\
\hline $\begin{array}{l}\text { Selective reporting (re- } \\
\text { porting bias) }\end{array}$ & Unclear risk & $\begin{array}{l}\text { Insufficient information to permit a judgement of high or low risk although it is } \\
\text { noted that some secondary outcomes, not related to the main research ques- } \\
\text { tion, are not reported by the study. }\end{array}$ \\
\hline Other bias & Unclear risk & $\begin{array}{l}\text { Inclusion criteria differ between protocol (mini TQ score over 12) and study } \\
\text { publications (mini TQ score over 8). }\end{array}$ \\
\hline
\end{tabular}

Kaldo 2007

Methods Two-arm, non-blinded, parallel-group RCT, with 6 weeks of treatment and 12 months follow-up

\section{Participants}

\section{Location: Sweden}

Setting of recruitment and treatment: participants were recruited into the study that ran from September 2002 to June 2003 via advertisements and articles in newspapers, via the Internet, and from a waiting list at the Department of Audiology at Uppsala University Hospital, Sweden. Treatment was a self-help CBT book complemented with weekly telephone calls.

\section{Sample size: 72}

- Number randomised: 34 in guided CBT bibliotherapy, 38 in wait list control

- Number completed: 30 in guided CBT bibliotherapy, 30 in wait list control

\section{Participant (baseline) characteristics:}

- Age: 45.9 years in guided CBT bibliotherapy, 48.5 years in wait list control

- Gender: 35 females (17 in guided CBT bibliotherapy, 18 in wait list control), 37 males (17 in guided CBT bibliotherapy, 20 in wait list control)

- Tinnitus duration: 8.6 years in guided CBT bibliotherapy, 12.4 years in wait list control 
Kaldo 2007 (Continued)

Inclusion criteria: potential participants 1) must have had a medical examination regarding tinnitus, which had been performed by an ear, nose and throat specialist or an audiological physician; 2 ) must have a tinnitus duration of at least 6 months; 3) must have an ability to read and understand the self-help book; 4) must be likely to complete the self-help process (e.g. participants were not expecting to travel abroad during the treatment or had any major medical or psychiatric condition); 5) must be above 18 years of age; 6) must have a score of 10 or above on the Tinnitus Reaction Questionnaire (TRQ); and 7) must have a score of 18 or below on both the anxiety and depression subscales of the Hospital Anxiety and Depression Scale (HADS)

Exclusion criteria: none reported

Interventions

Guided bibliotherapy CBT: the CBT self-help book's text and structure were largely derived from the Internet-administered self-help treatment developed at the Uppsala University Hospital Department of Audiology (Kaldo 2004a).

The book included: information about tinnitus, the distress it can lead to, and the aims and methods of CBT for tinnitus; planning the treatment and choosing among the available tools; defining treatment goals; making priorities to find time to spend on the treatment assignments; applied relaxation; positive imagery; focus exercises; exposure to tinnitus; sound enrichment; graded exposure to non-risk sounds; advice on how to cope with a hearing deficit; cognitive restructuring; CBT methods to deal with sleep problems; concentration management; evaluation and maintenance; summaries and evaluation of the treatment; planning on how to maintain positive effects; and relapse prevention. A scheduled phone call with the therapist marked the end of 1 week and the beginning of the next. All phone calls were with the same therapists and focused on evaluating treatment progress, providing advice on how to move on, and general feedback on progress.

The 2 therapists had 4.5 years of clinical psychologist training and in addition, received $6 \times 1$-hour long group supervisions by 2 licensed clinical psychologists with extensive experience of working with CBT for tinnitus.

Comparator: wait list control. The wait list control group received the self-help book and one initial phone call immediately after the participants in the treatment group had finished their treatment.

Use of additional interventions: none reported

Outcomes

Primary outcome: Tinnitus Reaction Questionnaire (TRQ)

Secondary outcomes: Tinnitus Handicap Inventory (THI), daily diary visual analogue scales relating to perceived loudness of tinnitus, distress caused by tinnitus, perceived stress during the day; Hospital Anxiety and Depression Scale-Anxiety (HADS-A) subscale, Hospital Anxiety and Depression-Depression subscale (HADS-D)

Measurement time points: pre and post treatment as well as 12-month follow-up data

Funding sources

Declarations of interest

This study was funded by a grant from the Swedish Hard of Hearing Association. The funders played no role in the study design, conduct or reporting (personal communication)

Notes

No conflicts of interest declared (personal communication)

Participants lost to follow-up: 12 (4 in the guided CBT bibliotherapy, 8 in the wait list control)

Adverse effects: none (personal communication)

Other: although the TRQ was the specified primary outcome measure for this study, the results for the $\mathrm{THI}$ were included in the meta-analysis as per the pre-specified protocol

\section{Risk of bias}

Bias Authors' judgement Support for judgement


Kaldo 2007 (Continued)

Random sequence genera- Low risk Quote: "... participants meeting the inclusion criteria were randomised to a tion (selection bias) treatment or a wait-list condition by means of coin tossing (performed by a person not otherwise related to the study)."

\begin{tabular}{|c|c|c|}
\hline $\begin{array}{l}\text { Allocation concealment } \\
\text { (selection bias) }\end{array}$ & Unclear risk & $\begin{array}{l}\text { Quote: "... participants meeting the inclusion criteria were randomised to a } \\
\text { treatment or a wait-list condition by means of coin tossing (performed by a } \\
\text { person not otherwise related to the study)." }\end{array}$ \\
\hline
\end{tabular}

\begin{tabular}{|c|c|c|}
\hline $\begin{array}{l}\text { Blinding of participants } \\
\text { and personnel (perfor- } \\
\text { mance bias) } \\
\text { All outcomes }\end{array}$ & High risk & $\begin{array}{l}\text { No blinding of participants or personnel to conditions (one of which was a wait } \\
\text { list). }\end{array}$ \\
\hline $\begin{array}{l}\text { Blinding of outcome as- } \\
\text { sessment (detection bias) } \\
\text { All outcomes }\end{array}$ & Unclear risk & $\begin{array}{l}\text { The outcome measures were self-reported/administered questionnaires, but } \\
\text { there was insufficient information available to permit a judgement of 'low risk' } \\
\text { or 'high risk'. }\end{array}$ \\
\hline
\end{tabular}

\begin{tabular}{|c|c|c|}
\hline $\begin{array}{l}\text { Incomplete outcome data } \\
\text { (attrition bias) } \\
\text { All outcomes }\end{array}$ & Low risk & $\begin{array}{l}\text { There was only a small amount of missing data and intention-to-treat analyses } \\
\text { was used. }\end{array}$ \\
\hline $\begin{array}{l}\text { Selective reporting (re- } \\
\text { porting bias) }\end{array}$ & Low risk & $\begin{array}{l}\text { The protocol was not available, but all the outcomes listed in the methods } \\
\text { were reported in the results. }\end{array}$ \\
\hline Other bias & Low risk & No other source of bias identified. \\
\hline
\end{tabular}

Kreuzer 2012

\begin{tabular}{ll}
\hline Methods & Two-arm, non-blinded, parallel-group RCT, with 22 weeks treatment and no follow-up \\
\hline Participants & Location: Aachen, Germany \\
& $\begin{array}{l}\text { Setting of recruitment and treatment: clients were recruited by direct referral from a local ENT physi- } \\
\text { cian of the University of Regensburg, Germany and by an advertisement in the newsletter of the Ger- } \\
\text { man Tinnitus League. Treatment was conducted in Aachen and the study conducted between April and } \\
\text { December 2010. }\end{array}$
\end{tabular}

\section{Sample size: 36}

- Number randomised: 18 in mindfulness, 18 in wait list control

- Number completed: 16 in mindfulness, 17 in wait list control

\section{Participant (baseline) characteristics:}

- Age: 49.6 years in mindfulness, 51.7 years in wait list control

- Gender: 17 females ( 7 in mindfulness, 10 in wait list control), 19 males (11 in mindfulness, 8 in wait list control)

- Tinnitus duration: 8.4 years in the mindfulness, 11.9 years in wait list control

Inclusion criteria: 1) age between 18 and 80 years; 2) location in the north-western part of Germany or in Belgium and the ability to understand the German language; 3 ) no communicational problems; 4) individual burden caused by subjective tinnitus for at least 6 months; and 5) absence of any unstable medical conditions

\section{Exclusion criteria: not reported} 2) imagination exercises, 3) self-massage and individualised gentle movement exercises of the body, 
4) exercises aiming at directing moment-to-moment awareness of body- and self-perception and 5) breathing exercises with emphasis on expiration in order to reduce muscle tension and increase relaxation Holl 2011.

One of the authors of the study $(\mathrm{MH})$ also wrote the therapy manual and conducted the therapy over 2 weekends ( 11 hours of treatment/weekend) with an interval of 7 weeks). Two weeks after each weekend and 11 and 15 weeks after the second training weekend patients gathered for a review meeting lasting 2 hours each. Patients were strongly encouraged to perform exercises themselves regularly and were instructed to contact and motivate each other by telephone at least once a week.

Comparator: wait list control. Participants in the wait list control group commenced therapy 24 weeks after baseline measurements.

Use of additional interventions: self-massage

$\begin{array}{ll}\text { Putcomes } & \text { Primary outcome: change in Tinnitus Questionnaire (TQ; German version) scores from baseline to } 9 \\ & \text { weeks } \\ & \text { Secondary outcomes: Tinnitus Handicap Inventory (THI), Beck Depression Inventory (BDI), tinnitus } \\ \text { numeric rating scales (loudness, discomfort, annoyance, distractibility and unpleasantness) }\end{array}$

Measurement time points: baseline and then weeks 7, 9 and 24

\section{Funding sources}

Declarations of interest
$\mathrm{MH}$ (one of the authors) was supported by a grant from the Bundesverband der Innungskrankenkassen (IKK), Association of Health Insurances. The authors were supported by a grant from the Tinnitus Research Initiative (TRI) to the Tinnitus Research Initiative Database.

$\mathrm{MH}$ wrote the treatment manual for "Tinnitus Atemtherapie" and offers this treatment in private practice. The other authors declare no competing interests in relation to this article.

Financial disclosure: "MH has written a book describing the methods of the applied behavioral techniques. The other authors have no conflicts of interest or disclosures to declare in relation to this article."

Other: although the change in the TQ score was the specified primary outcome measure for this study, the results for the THI were included in the meta-analysis as per the pre-specified protocol.

Treatment lasted 22 weeks but the post-treatment measure took place at 24 weeks.

\section{Risk of bias}

\begin{tabular}{|c|c|c|}
\hline Bias & Authors' judgement & Support for judgement \\
\hline $\begin{array}{l}\text { Random sequence genera- } \\
\text { tion (selection bias) }\end{array}$ & Low risk & $\begin{array}{l}\text { Randomisation was conducted by applying a computer-generated random } \\
\text { list. }\end{array}$ \\
\hline $\begin{array}{l}\text { Allocation concealment } \\
\text { (selection bias) }\end{array}$ & Unclear risk & Information not reported in the manuscript. \\
\hline $\begin{array}{l}\text { Blinding of participants } \\
\text { and personnel (perfor- } \\
\text { mance bias) } \\
\text { All outcomes }\end{array}$ & High risk & $\begin{array}{l}\text { No blinding, with participants in the wait list control having to wait } 24 \text { weeks } \\
\text { before treatment. }\end{array}$ \\
\hline $\begin{array}{l}\text { Blinding of outcome as- } \\
\text { sessment (detection bias) } \\
\text { All outcomes }\end{array}$ & High risk & $\begin{array}{l}\text { No blinding of outcome assessment and the outcome measurement was likely } \\
\text { to be influenced by lack of blinding. }\end{array}$ \\
\hline
\end{tabular}


Kreuzer 2012 (Continued)

Incomplete outcome data Unclear risk Low attrition and only complete cases reported, but the reasons for dropout (attrition bias) were not given.

All outcomes

Selective reporting (re- Low risk Outcomes reported fully and per protocol.
porting bias)

Other bias Unclear risk Waiting list controls have a risk of over-estimating the benefit of an intervention and the long duration of the wait list control could lead to spontaneous improvements.

Methods Three-arm, non-blinded, parallel-group RCT, with 2 to 3 weeks treatment and 2-week follow-up

Participants

Location: Uppsala, Sweden

Setting of recruitment and treatment: participants were recruited from patients who had been referred to the Department of Audiology in the University Hospital Uppsala for severe tinnitus. The interventions were conducted at the Department of Audiology and the study took place between January and November 1988.

\section{Sample size: 27}

- Number randomised: 9 to relaxation and exposure, 10 to relaxation and distraction, 8 to wait list control

- Number completed: 9 to relaxation and exposure, 10 to relaxation and distraction, 8 to wait list control

\section{Participant (baseline) characteristics:}

- Age: 51.2 years in the relaxation and exposure group, 61.5 in relaxation and distraction group, 52.1 years in wait list control

- Gender: 13 females ( 2 in relaxation and exposure, 7 in relaxation and distraction, 4 in wait list control), 14 males ( 7 in relaxation and exposure, 3 in relaxation and distraction, 4 in wait list control)

- Tinnitus duration: 10.8 years (total sample)

Inclusion criteria: 1) tinnitus should be the major otological complaint; 2) tinnitus should have been constantly present for at least 1 year; and 3) no other acute psychiatric or somatic disorder should be present

Exclusion criteria: underlying retro-cochlear pathology

Both interventions comprised $10 \times 1$-hour sessions over a period of 2 to 3 weeks and were given in an individual format by licensed psychologists ( 2 authors of the study).

The treatment was identical in the 2 interventions for the first 6 sessions. Both the relaxation and exposure $(\mathrm{RE})$ and the relaxation and distraction (RD) group underwent a behaviour analysis according to a procedure described in Sundell 1982. The patients also received training in progressive relaxation, differential

relaxation, quick relaxation according to procedures in Bernstein 1973, and were instructed to practise the skills they had been taught. The concluding 4 sessions of therapy differed between the 2 groups.

Relaxation and exposure: during the last 4 therapy sessions, participants were trained to cope with the tinnitus sound itself while being exposed to tinnitus-provoking environmental noise. Training was conducted in the clinic while the participants were sitting in a comfortable chair listening to prerecorded environmental noises. During the final training sessions the patients were instructed to direct their attention to their tinnitus sound, while at the same time they were exposed to the pre-recorded 
Lindberg 1989 (Continued)

environmental noise. When the patients were fully concentrated on their tinnitus, they were asked to relax quickly and turn their attention to the loudspeaker sound. This technique was gradually trained under more and more trying acoustic conditions in order to achieve a generalised coping strategy.

Thus, as the participants reported decreased tinnitus while coping, the sound was gradually diminished. For participants reporting increased tinnitus when exposed to noise, the reverse technique was applied.

Comparator: relaxation and distraction. The latter part of the treatment in the RD group consisted of in vivo training of coping in order to develop control over the annoyance and discomfort associated with tinnitus. Through the use of applied relaxation and cognitive strategies such as pleasant images, distraction from tinnitus was achieved. Information on relevant real-life situations was obtained from the behaviour analysis, in which the patient's discomfort from tinnitus was carefully analysed and on the basis of which alternative behaviours in problematic situations were suggested and discussed with each patient.

Comparator: wait list control. Participants in the wait list control group received regular care after the waiting period lapsed although no follow-up data was systematically collected (personal communication).

Use of additional interventions: none reported

Primary and secondary outcomes were not specified.
Outcome measures: visual analogue scales were used to measure on a daily basis for 1 week, subjec-
tive loudness, discomfort from tinnitus and ability to control the discomfort from tinnitus (participants
were instructed to complete these measures at the end of the day)

Measurement time points: 1 week before treatment and 2 weeks post-treatment

Funding sources

This study was supported financially by the Swedish Ministry of Health and Social Affairs, the Commission for Social Research (Project No. E 86,/168), and grants from Stiftelsen Tysta Skolan. The funders played no role in the design, conduct or reporting of the study (personal communication).

\begin{tabular}{ll}
\hline Declarations of interest & No conflicts of interest (personal communication) \\
\hline Notes & Participants lost to follow-up: 0 \\
& Adverse effects: none (personal communication) \\
& Other: outcomes and respective measures not suitable for inclusion in meta-analyses. \\
& Randomisation was made in blocks of $5(5+5+5)$ in order ensure equal distribution to groups (RE, RD, \\
& WLC) and therapist (BS, PL).
\end{tabular}

$\mathrm{WLC}$ ) and therapist (BS, PL).

\section{Risk of bias}

\begin{tabular}{lll}
\hline Bias & Authors' judgement & Support for judgement \\
\hline $\begin{array}{l}\text { Random sequence genera- } \\
\text { tion (selection bias) }\end{array}$ & Unclear risk & $\begin{array}{l}\text { Authors reported that a random list of number was used but could not recall } \\
\text { how it was generated (personal communication). }\end{array}$ \\
\hline $\begin{array}{l}\text { Allocation concealment } \\
\text { (selection bias) }\end{array}$ & Low risk & $\begin{array}{l}\text { Authors reported that allocations were in sealed envelopes that were opened } \\
\text { after participant accepted (personal communication). }\end{array}$ \\
\hline $\begin{array}{l}\text { Blinding of participants } \\
\text { and personnel (perfor- } \\
\text { mance bias) } \\
\text { All outcomes }\end{array}$ & Unclear risk & Information not reported in the manuscript. \\
\hline
\end{tabular}

Blinding of outcome as- Unclear risk Insufficient information to judge high or low risk. sessment (detection bias) 
Lindberg 1989 (Continued)

All outcomes

Incomplete outcome data Low risk Details of individuals provided: there was no dropout.
(attrition bias)

All outcomes

$\begin{array}{lll}\begin{array}{l}\text { Selective reporting (re- } \\ \text { porting bias) }\end{array} & \text { Unclear risk } & \begin{array}{l}\text { Insufficient information available to permit a judgement of 'low risk' or 'high } \\ \text { risk'. }\end{array}\end{array}$

Other bias Unclear risk Author reports no scientific input from funders but no protocol available.

\section{Malinvaud 2016}

Methods

Participants
Two-arm, non-blinded, parallel-group RCT, with 16-week duration of treatment and 3-month follow-up

Location: Paris, France

Setting of recruitment and treatment: patients were recruited and treated at the Tinnitus Clinic of the ENT Department, Hôpital Européen Georges Pompidou. Randomisation and treatment allocation took place between August 2009 and July 2011. Follow-up was completed in November 2011.

Sample size: 148

- Number randomised: 58 in CBT intervention, 61 in virtual reality comparison, 29 in wait list control

- Number completed: 44 in CBT intervention, 50 in virtual reality comparison, 25 in wait list control

Participant (baseline) characteristics:

- Age: 49.1 years in CBT intervention, 52.2 years in virtual reality comparison, 54.2 years in wait list control

- Gender: 43 females (19 in CBT intervention, 12 in virtual reality comparison, 12 in wait list control), 101 males, (36 in CBT intervention, 49 in virtual reality comparison, 16 in wait list control)

- Tinnitus duration: not reported

Inclusion criteria: age between 18 and 70 with informed consent signed, subjective tinnitus from peripheral aetiology (middle ear, inner ear, auditory nerve), stable and chronic tinnitus being present for at least 1 year, good language skills (understanding, ability to answer questionnaires), unilateral or perhaps define predominantly unilateral tinnitus with normal or slightly impaired hearing tinnitus with a well-defined spectrum that permits the fabrication of an exact copy that can be modulated in a virtual environment, and failure of the usual pharmacological agents (vasoactive drugs, anticonvulsants, etc.)

Exclusion criteria: fluctuating tinnitus, poor frequency matching, active Ménière's disease, contraindication to virtual reality environment techniques (claustrophobia, vision impairment), ongoing medical litigation, uncontrolled psychiatric pathologies and pregnancy

Interventions

CBT: CBT was provided in 2 steps. The first step started approximately 2 to 6 weeks after randomisation. It consisted of $2 \times 1$-hour sessions during which explanations regarding subjective tinnitus physiopathology and treatment modalities were presented, and short techniques of respiratory control and relaxation were taught.

The second step started 3 weeks after, and lasted for 12 weeks. Eight x 90-minute group treatment sessions, provided by a psychologist, were delivered in a 12-week period. CBT consisted of: psychoeducation, cognitive restructuring, exposure techniques, mindfulness-based elements, stress relief, applied relaxation, extensive explanation of neurophysiological model and fear avoidance discussion. Each session was ended with group discussion and questions were answered.

Comparator: virtual reality. Virtual reality (VR) was provided in 2 steps. The first step started approximately 2 to 6 weeks after randomisation. It consisted of $2 \times 1$-hour sessions during which explanations regarding subjective tinnitus physiopathology and treatment modalities were presented, and short 
Malinvaud 2016 (Continued)

techniques of respiratory control and relaxation were taught. The sessions lasted an hour. Each patient had 1 session per week for 8 weeks. During each session, the patients were given the ability to voluntarily and freely manipulate a sound resembling their individual tinnitus in a 3D auditory and visual virtual environment. Tinnitus avatar sound was virtually attached to the tip of a rod and its audio components were real-time spatialised according to the navigation and manipulation of the patient. Tinnitus avatar was also materialised as a sparkling spot in the 3D head-mounted display in order to facilitate multi-sensory integration. Patients were asked to navigate in 3 different environments (countryside, urban and indoor scenes) in each of which 10 different fixed sounds were present. Patients could then gain agency on tinnitus by displacing, masking or unmasking it at will.

The virtual reality protocol was directed by an ENT specialist, assisted by physicians specialised in virtual reality, biophysicists and hearing physiologists.

\section{Comparator: wait list control}

Use of additional interventions: none reported

Outcomes

Primary outcome: subjective Tinnitus Severity Scale

Secondary outcomes: Tinnitus Handicap Inventory (THI), Tinnitus Handicap Questionnaire (THQ), Hospital Anxiety and Depression Scale-Anxiety (HADS-A) subscale, Hospital Anxiety and Depression-Depression subscale (HADS-D)

Measurement time points: pre and 3 months after intervention

\begin{tabular}{ll}
\hline Funding sources & "This research is supported by a Tinnitus Research Initiative Grant (PB 07 01), by the French ANR RIAM \\
& 00402 "EarToy" and by AMPLIFON France."
\end{tabular}

Declarations of interest No conflicts of interest declared

Notes

Participants lost to follow-up: 25 (11 in CBT intervention, 11 in virtual reality comparison, 3 in wait list control).

Adverse effects: in the virtual reality comparison group, 3 people experienced "cybersickness" associated with the virtual reality and 1 person experienced an exacerbation of their tinnitus. There were no adverse effects in the CBT group.

Other: although the change in the subjective Tinnitus Severity Scale score was the specified primary outcome measure for this study, the results for the THI were included in the meta-analysis as per the hierarchy pre-specified in the protocol.

Unpublished data sought but not received for the subscales of the HADS and THI for both the control and intervention groups. Baseline data reflecting no between group differences were used in the metaanalysis in accordance with the conservative approach that we specified in the protocol that we would use in the event of missing data for included studies.

\section{Risk of bias}

\begin{tabular}{lll}
\hline Bias & Authors' judgement & Support for judgement \\
\hline $\begin{array}{l}\text { Random sequence genera- } \\
\text { tion (selection bias) }\end{array}$ & Low risk & Quotes: \\
& (From the article) \\
& "2.3. Randomization \\
& $\begin{array}{l}\text { Randomization and treatment allocation took place between August, 2009, } \\
\text { and July, 2011, and follow-up was completed in November, 2011. The ran- } \\
\text { domisation was performed with SAS system Version 9.2 (SAS Institute, Cary, } \\
\text { NC, USA) by a statistician independent of the study. The allocation was done } \\
\text { according a 2: 2: } 1 \text { ratio after screening and return of informed consent, i.e., } \\
\text { twice as many patients VR and CBT groups than the control group (WL). The }\end{array}$
\end{tabular}


randomisation was unstratified, and patients were randomised in blocks of five and ten patients randomly permuted."

(From person communication)

"The randomisation list was fixed beforehand. In each block of 10 patients there were two patients allocated to the waiting list, 4 to the virtual reality therapy and 4 to the CBT. Moreover in each block of five patient 1 was allocated to the waiting list, two to the virtual reality therapy and 2 to the CBT. When the informed consent signed the patient was assigned to the arm according to the next number of the randomisation list."

\begin{tabular}{lll}
\hline $\begin{array}{l}\text { Allocation concealment } \\
\text { (selection bias) }\end{array}$ & Low risk & $\begin{array}{l}\text { Quote: "The randomisation was performed with SAS system Version } 9.2 \text { (SAS } \\
\text { Institute, Cary, NC, USA) by a statistician independent of the study." }\end{array}$ \\
\hline $\begin{array}{l}\text { Blinding of participants } \\
\text { and personnel (perfor- } \\
\text { mance bias) }\end{array}$ & High risk & $\begin{array}{l}\text { Quote: "We undertook an open, and randomised controlled two arms trial } \\
\text { (RCT) comparing two juxtaposed therapeutic approaches." }\end{array}$ \\
All outcomes &
\end{tabular}

\begin{tabular}{|c|c|c|}
\hline $\begin{array}{l}\text { Blinding of outcome as- } \\
\text { sessment (detection bias) } \\
\text { All outcomes }\end{array}$ & High risk & $\begin{array}{l}\text { Self-report measures where allocation to treatment condition might have in- } \\
\text { fluenced responses. }\end{array}$ \\
\hline
\end{tabular}

\begin{tabular}{lll}
\hline $\begin{array}{l}\text { Incomplete outcome data } \\
\text { (attrition bias) } \\
\text { All outcomes }\end{array}$ & High risk & $\begin{array}{l}\text { Different were reasons given for dropout and missing data that appear to be } \\
\text { related to treatment outcome. }\end{array}$ \\
\hline $\begin{array}{l}\text { Selective reporting (re- } \\
\text { porting bias) }\end{array}$ & High risk & $\begin{array}{l}\text { The manuscript did not report the results for auditory sensitivity, which was } \\
\text { specified in the protocol. }\end{array}$ \\
\hline Other bias & Unclear risk & $\begin{array}{l}\text { Quote: "A few deviations from the initial protocol appeared during the study } \\
\text { as the number of patients randomised lower than what was planned (148 vs } \\
\text { 156), the questionnaire "hyperacusis" that wasn't distributed to the patients } \\
\text { because of an IT problem, and the use of the THI questionnaire that was not } \\
\text { planned initially." }\end{array}$ \\
\hline
\end{tabular}

\begin{tabular}{ll}
\hline Methods & Four-arm, non-blinded, parallel-group RCT, with 5 weeks of treatment and 4 weeks follow-up \\
\hline Participants & Location: Portland, USA \\
& Setting of recruitment and treatment: advertisements (flyers within the VAPORHCS, Internet, news- \\
paper) were used to recruit both veterans and civilians with tinnitus in the Portland metropolitan area. & Treatment was conducted at the NCRAR, which is a research centre at the Veterans Administration \\
& Healthcare System. The study was conducted between September 2015 and August 2016.
\end{tabular}

\section{Sample size: 40}

- Number randomised: 10 in CBT in intervention, 10 in ACT intervention, 10 in Coping Effectiveness Training (CET; active comparison), 10 in wait list comparison

- Number completed: 4 in CBT in intervention, 7 in ACT intervention, 6 in CET (active comparison), 10 in wait list comparison

\section{Participant (baseline) characteristics:}

- Age: 57.8 years overall 
Martz 2018 (Continued)

- Gender: 8 females, 32 males

- Tinnitus duration: not reported

Inclusion criteria: report experiencing tinnitus; a score of at least 21 on the Tinnitus Functional Index; 2 errors or less on a 6-item cognitive screening instrument; English-speaking; willing and able to give written informed consent; and use hearing aids if needed

Exclusion criteria: not meeting inclusion criteria; having previously participated in the PTM programme or one of the 2 focus groups held at the beginning of this pilot study; and having any other factor that would preclude full participation in the study

Interventions

The 3 group based interventions (CBT, ACT, CET) all included initial discussions of group rules, confidentiality, and personal introductions and were conducted by 2 co-leaders. Group leaders had clinical training in either psychology or counselling at doctoral level (2 leaders) and one at masters level. The $3 \times 2$-hour sessions featured PowerPoints and included the use of workbooks. Participants received an intervention-specific self-help workbook in the first session that they could keep.

CBT Intervention group: CBT treatments for tinnitus target the reduction of psychopathology by altering cognitive distortions, automatic thoughts and core beliefs, as well as behavioural techniques to reduce physiological arousal. Sessions included: the "CBT cycle" (cognitions/beliefs, emotions and behaviours); deep breathing and imagery relaxation exercises; positive activity scheduling, monitoring of positive activities; cognitive restructuring; discussion and identification of 12 common thought errors and examples related to tinnitus.

ACT intervention group: the ACT intervention aimed to reduce distress and resistance about having tinnitus and to increase committed actions based on one's values. The intervention included: struggling to stop negative reactions to tinnitus; concept of the observing the self and mindfulness; acknowledging negative thoughts and emotions; mindfulness exercises; cognitive diffusion; acceptance of internal experiences without attempting to control or change them; power of language; observing and being compassionate about unwanted internal experiences; exploration of life values; goal setting; and using ACT-T worksheet.

Comparator: Coping Effectiveness Training comparator (CET): CET aimed to increase understanding of stress and coping with tinnitus, and to better learn how to match appropriate coping strategies, based on whether the stressful situation is changeable or not. CET included: definitions of stress, types of stress (changeable or not, specific compared with general stress); problem solving strategies for types of stressful situations; assessing stress-relieving style; visualisation and relaxation exercise; physical activity for managing stress; remembering positive experiences and planning pleasant events; discussion of tinnitus as an invisible disability; types and obtaining social support; positive perspectives despite having tinnitus.

Comparator: wait list control group. The wait list control received the CET intervention at the end of the treatment period.

Use of additional interventions: all interventions also included 2 audiological sessions which involved explanations on why and how to use sound enrichment techniques, information on the types of sound that individuals can use in sound enrichment, how to create a sound plan for using various types of sound targeted toward specific problem situations related to tinnitus, and how to protect one's hearing from further damage.

Measurement time points: pre, post and 4 weeks follow-up

\begin{tabular}{ll}
\hline Funding sources & Pilot study grant from the Veterans Affairs Rehabilitation Research \& Development fund \\
\hline Declarations of interest & Not reported \\
\hline Notes & $\begin{array}{l}\text { Participants lost to follow-up: } 13 \text { (6 from CBT intervention, } 3 \text { from ACT, 4 from CET). Intention-to-treat } \\
\text { analysis conducted. }\end{array}$ \\
\hline
\end{tabular}


Martz 2018 (Continued)

\section{Adverse effects: none}

Other: study data not included in any meta-analysis

\section{Risk of bias}

\begin{tabular}{lll}
\hline Bias & Authors' judgement & Support for judgement \\
\hline $\begin{array}{l}\text { Random sequence genera- } \\
\text { tion (selection bias) }\end{array}$ & Low risk & $\begin{array}{l}\text { Quote: "A random number generator was used, blocking by } 10 \text { per group to as- } \\
\text { sure equal and balanced numbers in each group. Randomization software pro- } \\
\text { vided the randomisation sequence." }\end{array}$
\end{tabular}

\begin{tabular}{lll}
\hline $\begin{array}{l}\text { Allocation concealment } \\
\text { (selection bias) }\end{array}$ & Low risk & $\begin{array}{l}\text { Quote: "Allocation concealment was achieved by using the sequentially num- } \\
\text { bered, opaque, sealed envelopes method." }\end{array}$ \\
\hline $\begin{array}{l}\text { Blinding of participants } \\
\text { and personnel (perfor- } \\
\text { mance bias) }\end{array}$ & High risk & $\begin{array}{l}\text { Quote: "Participants were not blinded to intervention assignments because } \\
\text { they had to be notified about their group assignment." }\end{array}$ \\
All outcomes & No information provided to suggest personnel blinding.
\end{tabular}

\begin{tabular}{|c|c|c|}
\hline $\begin{array}{l}\text { Blinding of outcome as- } \\
\text { sessment (detection bias) } \\
\text { All outcomes }\end{array}$ & Unclear risk & $\begin{array}{l}\text { Although the outcome measures were self-reported questionnaires, no infor- } \\
\text { mation was provided to inform judgement on whether outcomes were likely to } \\
\text { be affected. }\end{array}$ \\
\hline
\end{tabular}

All outcomes mation was provided to inform judgement on whether outcomes were likely to affected.

$\begin{array}{ll}\text { Incomplete outcome data } & \text { Unclear risk } \\ \text { (attrition bias) } & \text { reasons for attrition are not reported. }\end{array}$

All outcomes

Large amounts of missing data but intention-to-treat analysis employed. The

\begin{tabular}{|c|c|c|}
\hline $\begin{array}{l}\text { Selective reporting (re- } \\
\text { porting bias) }\end{array}$ & High risk & $\begin{array}{l}\text { Tinnitus Coping Style Questionnaire listed as a primary outcome in the trial } \\
\text { registration but not mentioned in the study publication. Tinnitus Functional } \\
\text { Index listed as a secondary outcome in the trial registration but not mentioned } \\
\text { in the study publication. }\end{array}$ \\
\hline
\end{tabular}

Other bias High risk

Repeated modifications to the study protocol that are not justified or clarified in the publication. For example, the initial anticipated sample size was 80 but only 40 included in final publication. ACT was only added as an intervention arm 1 year after initial registration.

McKenna 2017

Methods $\begin{aligned} & \text { Two-arm, non-blinded, parallel-group RCT, with 8-week duration of treatment and 6-month duration of } \\ & \text { follow-up }\end{aligned}$

Participants Location: London, England

Setting of recruitment and treatment: recruitment took place between January 2013 and March 2015 and treatment was conducted at Royal National Throat, Nose and Ear Hospital, London

\section{Sample size: 75}

- Number randomised: 39 in MBCT, 36 in relaxation

- Number completed: 34 in MBCT, 28 in relaxation

\section{Participant (baseline) characteristics:}

- Age: 47 in MBCT, 53 in relaxation (median)

- Gender: 34 females (18 in MBCT, 16 in relaxation), 41 males (21 in MBCT, 20 in relaxation)

- Tinnitus duration: 8 years in MBCT, 2.8 years in relaxation. 
Inclusion criteria: 1 ) aged 18 years or over; 2) reported tinnitus of more than 6 months' duration; 3) reported clinical levels of psychological distress (Clinical Outcomes in Routine Evaluation - Non-Risk, CORE-NR score > 10); 4) completed medical investigations for tinnitus; and 5) sufficient command of English and hearing levels allowing participation in group discussions

Exclusion criteria: 1) current, comorbid, severe physical or mental illness; 2) current risk factors of active suicidal ideation or self-harm; 3) current substance dependence

Interventions

Mindfulness Based Cognitive Therapy (MBCT). Mindfulness Based Cognitive Therapy (MBCT) with tinnitus-related modifications was delivered in groups ( $8 \times 120$-minute sessions) in line with manuals based on the protocol for depression (Segal 2012), by 2 clinical psychologists. Modifications involved introducing awareness of sounds from session 2 onwards, explicitly including sounds and tinnitus in group discussions and practices, teaching about the cognitive model of tinnitus (McKenna 2014), and showing videos of people with tinnitus who had completed MBCT. All sessions involved learning specific mindfulness meditations followed by discussions exploring participants' experiences ('inquiry'). Every session included homework review, group discussion and some psychoeducation. Some sessions included stories, videos and poetry. The MBCT group received supporting literature in the form of a workbook that summarised the material covered in the session.

Comparator: Relaxation Therapy (RT). Relaxation Therapy was based on standardised interventions for applied relaxation (Ost 1987), adapted to create an 8-week course for comparability to MBCT. RT was delivered in groups ( $8 \times 120$-minute sessions) by 2 clinical psychologists and involved formal experiential exercises (either relaxation or meditation), discussion about the exercises with a focus on trouble-shooting and planning ways to practise at home, and psychoeducation within the group. Psycho-education in RT focused on the physiology of stress and tinnitus and participants were asked to complete equivalent (to the MBCT group) amounts of daily formal practice (supported by audio guides) and to begin to apply their practice of either mindfulness or relaxation to daily life. RT participants did not receive supporting literature.

Use of additional interventions: none

Outcomes Primary outcome: pre- to post-treatment Tinnitus Questionnaire (TQ)

Secondary outcomes: additional time points of change on the TQ (i.e. baseline to pre-treatment, pretreatment to 1-month and 6-month follow-up); perceived tinnitus loudness on a $10 \mathrm{~cm}$ visual analogue scale; Tinnitus Functional Index (TFI); Hospital Anxiety and Depression Scale-Anxiety (HADS-A) subscale, Hospital Anxiety and Depression-Depression subscale (HADS-D); Tinnitus Catastrophizing Scale (TCS); Tinnitus Fear Avoidance Scale (T-FAS); Tinnitus Acceptance Questionnaire (TAQ); Mindful Attentions Awareness Scale (MAAS); Work and Social Adjustment Scale (WSAS).

Measurement time points: pre, post, 1 and 6 months follow-up

Funding sources British Tinnitus Association (BTA). The study sponsor did not play a role in the study design, data collection, analysis, interpretation or write-up.

Declarations of interest The authors declare that they have no conflicts of interest.

Notes Participants lost to follow-up: 13 (5 in intervention group, 8 in comparison)

Adverse effects: 2 adverse effects were reported in the course of the study although neither were considered to be related to the interventions

Other: "modified" intention-to-treat analysis conducted

The intervention and comparison groups were run on the same day with counterbalancing of morning/afternoon delivery.

\section{Risk of bias}

Bias Authors' judgement Support for judgement


McKenna 2017 (Continued)

Random sequence genera- Low risk Quote: "They were randomly allocated to RT or MBCT by computer." tion (selection bias)

\begin{tabular}{|c|c|c|}
\hline $\begin{array}{l}\text { Allocation concealment } \\
\text { (selection bias) }\end{array}$ & Low risk & $\begin{array}{l}\text { Quote: "They were randomly allocated to RT or MBCT by computer. Random- } \\
\text { ization was stratified by age and gender, conducted independently for each } \\
\text { cohort group to ensure later allocation sequence was not affected. Allocations } \\
\text { were sent to the trial clinical psychologists (L.M. and E.M.M.) who informed } \\
\text { participants } 4 \text { weeks prior to commencing treatment." }\end{array}$ \\
\hline
\end{tabular}

Blinding of participants Unclear risk and personnel (performance bias)

All outcomes
Quote: "It was not possible to mask the participants or clinicians to allocation, although participants were masked to the content of the alternative treatment."

Insufficient information provided to make a judgement on high or low risk of bias.

Blinding of outcome as-
sessment (detection bias)

Unclear as the person conducting randomisation also analysed the data and was only blinded for the initial analyses.

Quote: "Independent statisticians analysing the results were masked to group for the initial analyses."

The method of questionnaire administration is unclear.

\begin{tabular}{|c|c|c|}
\hline $\begin{array}{l}\text { Incomplete outcome data } \\
\text { (attrition bias) } \\
\text { All outcomes }\end{array}$ & Unclear risk & $\begin{array}{l}\text { There is some concern about the proportion of missing data and the clarity } \\
\text { with which it is reported, making it difficult to judge 'high' or 'low' risk. Last } \\
\text { observation carried forward was used to replace the missing data. }\end{array}$ \\
\hline
\end{tabular}

Selective reporting (re- Low risk $\quad$ Outcomes reported match those specified in the protocol.
Outcomes reported match those specified in the protocol.

Other bias Low risk The study appears to be free of other sources of bias.

Nyenhuis 2013a

\begin{tabular}{ll}
\hline Methods $\quad$ Four-arm, non-blinded, parallel-group RCT, with 3-month duration of treatment and 9-month follow-up \\
\hline
\end{tabular}

\section{Location: Germany}

Setting of recruitment and treatment: participants were recruited via newspapers, radio, ENT offices and outpatient clinics. The assessment was conducted at 2 study centres in the southern region of Lower Saxony (Germany).

\section{Sample size: 304}

- Number randomised: 71 in Group CBT, 79 in Internet-based self-help intervention, 77 in bibliotherapy self-help intervention, 77 in information only comparison

- Number completed: 47 in Group CBT, 44 in Internet-based self-help intervention, 45 in bibliotherapy self-help intervention, 49 in information only comparison

\section{Participant (baseline) characteristics:}

- Age: 50.1 years in the Group CBT intervention, 47.8 years in Internet-based self-help intervention, 45.8 years in the bibliotherapy self-help intervention, 50.4 in the information only comparison, 48.5 overall

- Gender (\% female): 46.5 in the Group CBT, 47.4 in Internet-based self-help intervention, 44.2 in bibliotherapy self-help intervention, 42.9 in the information only comparison, 45.4 overall

- Tinnitus duration: 3.2 months in Group CBT, 3 months in Internet-based self-help, 3.3 months in bibliotherapy self-help intervention, 3.2 months in the information only comparison, 3.2 months overall 
Inclusion criteria: a participant was included if he or she had experienced idiopathic tinnitus for 2 to 26 weeks, was between 18 and 75 years old and was not receiving any other tinnitus-related psychological treatment; able to access the Internet and potentially be able to take part in weekly group sessions to ensure that patients could be randomly allocated to each condition

Exclusion criteria: not reported

Group CBT: contents of the training were presented by 3 psychologists (one of whom had previously conducted Group CBT for tinnitus patients) in $4 \times 2$-hour meetings, with each meeting including progressive muscle relaxation. At the end of the first session, participants received a CD with the progressive muscle relaxation instructions and information booklet. The sessions included information and discussion about: the neuro-acoustical and psychological model of tinnitus perception and the tinnitus distress response; coping with tinnitus; attention diversion; cognitive reappraisal; progressive muscle relaxation; general stress management; and information on sleep hygiene.

Self-help comparators: the 2 self-help comparators were based on the same manual, adapted from the CBT-oriented Tinnitus Coping Training (Kröner-Herwig 1997; Kröner-Herwig 2003). The text presented central concepts regarding the neuro-acoustical and psychological model of tinnitus perception and the tinnitus distress response. As means of coping with tinnitus, attention diversion, cognitive reappraisal, progressive muscle relaxation and general stress management were explained. An optional chapter/module provided information on sleep hygiene and coping techniques.

At regular intervals, the manual/modules contained multiple-choice self-tests to check text comprehension. At the end of each training lesson, exercises were offered to participants to practise the suggested self-management skills (e.g. to find and develop ways out of one's personal vicious circle of tinnitus distress, or to deliberately concentrate on other perceptual input such as the smell of coffee or surrounding noises).

Features unique to the comparator Internet-based self-help: contents of the manual were written as web pages and participants could download the progressive muscle relaxation instructions as an MP3-data file in order to use it offline.

Features unique to the comparator bibliotherapy self-help: bibliotherapy consisted of a complete self-help, manual with instructions for progressive muscle relaxation presented on CD.

Comparator: information only. The participants in the information only condition received an 11page booklet that provided information on the morphological and functional characteristics of the auditory system, the potential triggers of tinnitus and medical treatment options. No treatment recommendations were given and all information was avoided that could instil optimistic or pessimistic thoughts about the prognosis of tinnitus. These participants received no further treatment.

Use of additional interventions: none reported

Outcomes Primary outcome: German version of the Tinnitus Questionnaire (TQ)

Secondary outcomes: Von Zerssen's symptom list was used to measure psychosomatic discomfort, Patient Health Questionniare-Depression subscale (German version)

Measurement time points: pre, post and 9 months follow-up

\begin{tabular}{ll}
\hline Funding sources & Federal Ministry of Research and Education, Germany (FKZ 01GX0732) \\
\hline Declarations of interest & None reported \\
\hline Notes & $\begin{array}{l}\text { Participants lost to follow-up: } 119 \text { (24 from Group CBT intervention, } 35 \text { from Internet-based self-help } \\
\text { intervention, 32 from bibliotherapy self-help intervention, } 28 \text { from information only comparison) }\end{array}$ \\
& $\begin{array}{l}\text { Adverse effects: referring to the intention-to-treat analysis for the reliable change results, } 1 \text { person in } \\
\text { the Group CBT deteriorated and } 2 \text { people in the information only condition deteriorated }\end{array}$
\end{tabular}

\section{Risk of bias}


Nyenhuis 2013a (Continued)

\begin{tabular}{|c|c|c|}
\hline Bias & Authors' judgement & Support for judgement \\
\hline $\begin{array}{l}\text { Random sequence genera- } \\
\text { tion (selection bias) }\end{array}$ & Low risk & $\begin{array}{l}\text { Quote: "The random sequence of study arms was generated at each study cen- } \\
\text { tre before data acquisition using computerised random number generators." }\end{array}$ \\
\hline $\begin{array}{l}\text { Allocation concealment } \\
\text { (selection bias) }\end{array}$ & Low risk & $\begin{array}{l}\text { Quote: "A student assistant, who was blind to the participant's identity and } \\
\text { pre-assessment data, assigned the codes to the study arm indicated by a ran- } \\
\text { dom sequence." }\end{array}$ \\
\hline $\begin{array}{l}\text { Blinding of participants } \\
\text { and personnel (perfor- } \\
\text { mance bias) } \\
\text { All outcomes }\end{array}$ & Unclear risk & Insufficient information to judge high or low risk. \\
\hline $\begin{array}{l}\text { Blinding of outcome as- } \\
\text { sessment (detection bias) } \\
\text { All outcomes }\end{array}$ & Unclear risk & Insufficient information to judge high or low risk. \\
\hline $\begin{array}{l}\text { Incomplete outcome data } \\
\text { (attrition bias) } \\
\text { All outcomes }\end{array}$ & Unclear risk & $\begin{array}{l}\text { Dropout rates higher for the self-help treatment conditions compared with the } \\
\text { group therapy, however the reasons for dropout were not explored or report- } \\
\text { ed. }\end{array}$ \\
\hline $\begin{array}{l}\text { Selective reporting (re- } \\
\text { porting bias) }\end{array}$ & Low risk & All outcomes reported in full. \\
\hline Other bias & Low risk & The study appears to be free of other sources of bias. \\
\hline
\end{tabular}

Oron (unpublished)

Methods Three-arm, non-blinded, parallel-group RCT, with 4 weeks of treatment and 1-month follow-up

Participants Location: Tel-Aviv, Israel

Setting of recruitment and treatment: the study was conducted in 2017 and participants were treated in a hospital setting, in Tel-Aviv

\section{Sample size: 45}

- Number randomised: 17 to ACT, 17 to Coping Effectiveness Training (CET), 11 to wait list control

- Number completed: 10 ACT, 9 Coping Effectiveness Training, 8 wait list control

\section{Participant (baseline) characteristics:}

- Age: 51.3 years (overall)

- Gender: 22 females, 23 males

- Tinnitus duration: not reported

Inclusion criteria: 1) reported experience of tinnitus; 2) agreeing to undergo a clinical assessment to determine if tinnitus regularly impaired emotion, cognition, attention and/or task-performance, and if it occurred in several situations (using tinnitus assessment guidelines proposed by Biesinger 1998; 3) being fluent in Hebrew; 4) willing and able to give written informed consent; and 5) using hearing aids if needed

Exclusion criteria: 1) not meeting inclusion criteria 1 to 5 ; and 2) having any other factor that the researchers viewed as prohibiting individuals from fully participating in the study 
Oron (unpublished) (Continued)

Interventions

All participants received one audiological information and then 3 sessions in their respective intervention groups. Sessions were conducted weekly, lasted up to a maximum of 2 hours and had a maximum of 10 participants assigned. (No information was available at this time about the intervention providers).

ACT intervention group: the ACT intervention aimed to reduce distress and resistance about having tinnitus and to increase committed actions based on one's values. The intervention included: struggling to stop negative reactions to tinnitus; concept of the observing the self and mindfulness; acknowledging negative thoughts and emotions; mindfulness exercises; cognitive diffusion; acceptance of internal experiences without attempting to control or change them; power of language; observing and being compassionate about unwanted internal experiences; exploration of life values; goal setting; and using ACT-T worksheet.

Comparator: Coping Effectiveness Training. CET aimed to increase understanding of stress and coping with tinnitus, and to better learn how to match appropriate coping strategies, based on whether the stressful situation is changeable or not. CET included: definitions of stress, types of stress (changeable or not, specific compared with general stress); problem solving strategies for types of stressful situations; assessing stress-relieving style; visualisation and relaxation exercise; physical activity for managing stress; remembering positive experiences and planning pleasant events; discussion of tinnitus as an invisible disability; types and obtaining social support; positive perspectives despite having tinnitus.

Comparator: wait list control. The wait list control received the CET intervention at the end of the treatment period.

Use of additional interventions: none

$\begin{array}{ll}\text { Outcomes } & \text { Primary and secondary outcome not distinguished. } \\ & \text { Outcome measures included: COPE questionnaire, Tinnitus Handicap Inventory (THI) } \\ \text { Measurement time points: pre, post and } 1 \text { month after treatment }\end{array}$

Funding sources $\quad$ Fulbright Commission, US Department of State

Declarations of interest No conflicts of interest (personal communication)

Notes Participants lost to 1-month follow-up: 18 (7 from ACT, 8 from CET, 3 from wait list control)

Adverse effects: none (personal communication)

Other: the ACT and CET interventions are the same as applied/used in Martz 2018

\begin{tabular}{lll}
\hline Risk of bias & & \\
\hline Bias & Authors' judgement & Support for judgement \\
\hline $\begin{array}{l}\text { Random sequence genera- } \\
\text { tion (selection bias) }\end{array}$ & Low risk & $\begin{array}{l}\text { Quote: "A random number generator was used to provide the randomisation } \\
\text { sequence." }\end{array}$ \\
\hline $\begin{array}{l}\text { Allocation concealment } \\
\text { (selection bias) }\end{array}$ & Low risk & $\begin{array}{l}\text { Quote: "Allocation concealment was achieved by using the sequentially num- } \\
\text { bered, opaque, sealed envelopes (SNOSE) method (Doig \& Simpson, 2005; } \\
\text { Schulz \& Grimes, 2002)." }\end{array}$ \\
\hline $\begin{array}{l}\text { Blinding of participants } \\
\text { and personnel (perfor- } \\
\text { mance bias) } \\
\text { All outcomes }\end{array}$ & High risk & $\begin{array}{l}\text { Quote: "Participants were not blinded to intervention assignments as they } \\
\text { were notified about their group assignment after the randomisation and allo- } \\
\text { cation processes were completed." }\end{array}$ \\
\hline
\end{tabular}

\footnotetext{
Blinding of outcome as-

Unclear risk

Insufficient information available to judge high or low risk.

sessment (detection bias)

All outcomes
} 
Oron (unpublished) (Continued)

Incomplete outcome data Unclear risk Insufficient information available to judge high or low risk. (attrition bias)

All outcomes

\begin{tabular}{|c|c|c|}
\hline $\begin{array}{l}\text { Selective reporting (re- } \\
\text { porting bias) }\end{array}$ & Unclear risk & $\begin{array}{l}\text { Insufficient information available to judge high or low risk as the manuscript is } \\
\text { currently in preparation. }\end{array}$ \\
\hline
\end{tabular}

Other bias Unclear risk Insufficient information available to judge high or low risk.

Methods Two-arm, non-blinded, parallel-group RCT, with 6 weeks of treatment and 3 months follow-up

\section{Participants}

Location: Louvain-la-Neuve, Belgium

Setting of recruitment and treatment: the study was advertised in local newspapers as a controlled clinical trial aimed at reducing the psychological distress resulting from tinnitus. Treatment was provided in a university psychology department.

\section{Sample size: 30}

- Number randomised: 15 to $\mathrm{MBCT}, 15$ to relaxation

- Number completed: 13 in MBCT, 12 in relaxation

\section{Participant (baseline) characteristics:}

- Age: 60.9 years in MBCT, 59.8 years in relaxation

- Gender: 12 females (6 in MBCT, 6 in relaxation), 18 males (9 in MBCT, 9 in relaxation)

- Tinnitus duration: not reported

Inclusion criteria: 1) tinnitus experienced within the past 6 months; 2 ) a medical check-up by a physician specialised in hearing disorders; 3 ) sufficient hearing capacity to follow instructions delivered during group sessions; and 4) significant psychological distress and impairment in everyday activities resulting from tinnitus

Exclusion criteria: 1) tinnitus resulting from an organic condition that could benefit from a medical intervention; 2) use of a tinnitus masking apparatus; 3) other psychotherapy or psychological intervention during the study; and 4) borderline or antisocial personality disorder choeducation. The treatments (MBCT or relaxation) consisted of $6 \times 40$-minute, weekly group sessions. The 2 groups were trained in parallel, at the same time, in similar, adjacent rooms within a university psychology department. The groups were instructed by $2 \mathrm{PhD}$ level psychotherapists, with at least 3 years of practice in mindfulness and in relaxation training, and with a formal training in MBCT. To avoid therapist effects, each instructor alternated in teaching the relaxation or the mindfulness session. Thus, each treatment group received the same amount of training from both instructors.

Mindfulness Based Cognitive Therapy: the intervention manual was derived from Segal 2012 and was adapted to treating tinnitus in the following ways. First, the content relative to psychoeducation of depression relapse was deleted, as the present treatment target was the psychological consequences of tinnitus. Second, the number of session was reduced from 8 to 6 . The first 4 sessions were very similar to the original programme: they comprised exactly the same exercises but a) referred to dealing with the adversity of tinnitus rather than with depression relapse and b) did not present the psycho-educative part of session 4. The fifth session of the programme merged aspects of sessions 5 and 6 of the original programme, focusing mostly on the theme that thoughts are not facts. The main exercise consisted of a 40-minute sitting meditation with a sequential focus on breath, body, thoughts and finally the introduction of a difficult thought in the meditation. The 6 th session of the programme merged aspects 
Philippot 2012a (Continued)

of sessions 7 and 8 of the original programme dealing with how to take care of oneself (relapse prevention) and evaluating the programme. The main exercise consisted in a 40-minute body scan.

Comparator: relaxation training. The manual followed the progressive relation training of Jacobson 1957 adapted by Van Rillaer 1997. The first session consisted of breathing training, the second session taught Jacobson relaxation and was divided into 13 body parts, the third session into 8 body parts, the 4 th session into 4 body parts and the fifth session into 2 body parts. The 6 th session focused on mini-relaxation and on maintenance of relaxation competence.

Use of additional interventions: psychoeducation was delivered in single 2-hour group sessions offered to multiple groups (about 10 participants in each group). Two psychologists informed the participants about the nature and characteristics of tinnitus, its epidemiology, aetiology as well as adaptive and ineffective coping strategies. An independent observer ensured that the information presented was consistent with the manual's content. Participants received a booklet outlining the information provided during the psychoeducation session as well as a 2-page self-observation report sheet on which to record their emotions, thoughts and behaviours associated with tinnitus.

\section{Outcomes Primary and secondary outcomes not distinguished.}

Outcome measures included: Tinnitus Psychological Impact Questionnaire (QIPA); State Trait Anxiety Inventory (STAI); Beck Depression Inventory (BDI)

Measurement time points: baseline, pre, post and 3 months after treatment
This research was supported by a grant from the Fonds National de la Recherche Scientifique de Belgique (grant no. 8.4505.00)

There were no conflicts of interest (personal communication)

\begin{tabular}{ll}
\hline Declarations of interest & There were no conflicts of interest (personal communication) \\
\hline Notes & Participants lost to follow-up: 5 (2 from MBCT, 3 from relaxation) \\
& Adverse effects: none \\
& $\begin{array}{l}\text { Other: QIPA is an unpublished 42-item measure that assesses } 6 \text { dimensions of tinnitus experience dur- } \\
\text { ing the past week. These outcome data were not included in the meta-analysis. }\end{array}$
\end{tabular}

\section{Risk of bias}

\begin{tabular}{|c|c|c|}
\hline Bias & Authors' judgement & Support for judgement \\
\hline \multirow[t]{2}{*}{$\begin{array}{l}\text { Random sequence genera- } \\
\text { tion (selection bias) }\end{array}$} & Unclear risk & $\begin{array}{l}\text { Although the manuscript reports that participants were randomly allocated, } \\
\text { there is no information on how the sequence was generated. }\end{array}$ \\
\hline & & $\begin{array}{l}\text { Quote: "Selected participants were randomly allocated to the mindfulness } \\
\text { training condition or to the relaxation training condition with the restriction } \\
\text { that there were equal numbers and gender proportions in each group: Within } \\
\text { gender, an equal number of participants was randomly allocated to each con- } \\
\text { dition." }\end{array}$ \\
\hline $\begin{array}{l}\text { Allocation concealment } \\
\text { (selection bias) }\end{array}$ & Unclear risk & Information not reported in the manuscript. \\
\hline $\begin{array}{l}\text { Blinding of participants } \\
\text { and personnel (perfor- } \\
\text { mance bias) } \\
\text { All outcomes }\end{array}$ & Unclear risk & Information not reported in the manuscript. \\
\hline $\begin{array}{l}\text { Blinding of outcome as- } \\
\text { sessment (detection bias) } \\
\text { All outcomes }\end{array}$ & Unclear risk & $\begin{array}{l}\text { The outcome measures were self-reported/administered questionnaires, but } \\
\text { there was insufficient information available to permit a judgement of low risk } \\
\text { or high risk. }\end{array}$ \\
\hline
\end{tabular}


Philippot 2012a (Continued)

Incomplete outcome data Low risk (attrition bias)

All outcomes
Five participants did not complete the study or were excluded from the analysis; the reasons given for incomplete data were obtained and similar across groups (i.e. excluded due to not attending sessions, or not returning outcome measures).

\begin{tabular}{lll}
\hline $\begin{array}{l}\text { Selective reporting (re- } \\
\text { porting bias) }\end{array}$ & Low risk & $\begin{array}{l}\text { Although there is no protocol available, all outcomes mentioned in the meth- } \\
\text { ods section were reported. }\end{array}$ \\
\hline Other bias & Low risk & No other sources of bias identified. \\
\hline
\end{tabular}

\section{Robinson 2008}

\section{Methods}

Participants

Two-arm, non-blinded, parallel-group RCT, with 8 weeks of treatment and 8, 16 and 52 weeks follow-up

Location: California, USA

Setting of recruitment and treatment: recruitment and treatment took place in a Veterans Affairs hospital, San Diego between 1998 and 2001

\section{Sample size: 65}

- Number randomised: 38 in CBT, 27 in wait list control

- Number completed: 26 in CBT, 15 in wait list control

\section{Participant (baseline) characteristics:}

- Age: 55.0 years for entire sample

- Gender: $48 \%$ of the entire sample was female

- Tinnitus duration: 9.9 years in CBT, 11.2 years in wait list control

Inclusion criteria: the primary inclusion criterion was self-reported distress due to tinnitus; no level of severity or frequency of tinnitus was required

Exclusion criteria: factors that interfered with patient ability to participate in a group for physical (e.g. unable to get to group) or psychological reasons (e.g. psychosis or dementia)

Interventions

CBT: the 8-session CBT intervention was based on a CBT group treatment manual for depression (Munoz 1993), and delivered by a psychiatrist and doctoral psychology students in a group format. The intervention emphasised cognitive restructuring, an increase in pleasant activities and relaxation training. The specific topics addressed in the 8 weeks were: thoughts, behaviours, stress and tinnitus in week 1 ; increasing pleasant activities in week 2 ; relaxation techniques in week 3 ; cognitive restructuring in weeks 4 to 6; goal setting in week 7 ; and summary and review in week 8 . The interventions in this study were modified to make them relevant to coping with tinnitus and stress in general and were also modified by the authors by adding relaxation techniques. Homework assignments were given weekly and reviewed during the next session.

Comparator: wait list control

Use of additional interventions: none reported

Outcomes

\section{Primary and secondary outcomes were not specified.}

The outcome measures included: Tinnitus Handicap Questionnaire (THQ); Tinnitus Reaction Questionnaire (TRQ); Tinnitus Handicap Inventory (THI); Tinnitus Questionnaire (TQ); Modified Somatic Perception Questionnaire (MSPQ); Hamilton Rating Scale for Depression (HRSD); Beck Depression Inventory (BDI); Symptom Checklist 90, revised (SCL-90-R); Quality of Well-being Scale, self-administered version (QWB-SA; Kaplan 1996) 
Robinson 2008 (Continued)

Measurement time points: pre, post, 16 and 52 weeks

\begin{tabular}{ll}
\hline Funding sources & The American Tinnitus Association funded some of the study, but had no role in the study. \\
\hline Declarations of interest & No conflicts of interest. \\
\hline
\end{tabular}

Notes Participants lost to follow-up: 24 out of 65 participants dropped out (37\%); 12 were from the intervention group and 12 were from the comparison group

Adverse effects: none (personal communication)

Other: the treatment manual is available from the first author.

Specific data for the outcome measures all time points except for pre-treatment are not available and thus the study is not included in the meta-analyses.

\section{Risk of bias}

\begin{tabular}{|c|c|c|}
\hline Bias & Authors' judgement & Support for judgement \\
\hline $\begin{array}{l}\text { Random sequence genera- } \\
\text { tion (selection bias) }\end{array}$ & Low risk & $\begin{array}{l}\text { Quote: "Random assignment was made using a random number generator, } \\
\text { and each participant had an equal chance of being assigned to begin treat- } \\
\text { ment either immediately or after an 8-week waiting period." }\end{array}$ \\
\hline $\begin{array}{l}\text { Allocation concealment } \\
\text { (selection bias) }\end{array}$ & Low risk & $\begin{array}{l}\text { Quote: "Neither the staff nor the participant knew to which arm the participant } \\
\text { was randomly assigned until after completion of initial assessments." }\end{array}$ \\
\hline $\begin{array}{l}\text { Blinding of participants } \\
\text { and personnel (perfor- } \\
\text { mance bias) } \\
\text { All outcomes }\end{array}$ & High risk & No blinding of participants or personnel after the initial assessments. \\
\hline $\begin{array}{l}\text { Blinding of outcome as- } \\
\text { sessment (detection bias) } \\
\text { All outcomes }\end{array}$ & Unclear risk & $\begin{array}{l}\text { Although the outcome measures were self-reported/administered question- } \\
\text { naires, there was insufficient information available to permit a judgement of } \\
\text { low risk or high risk. }\end{array}$ \\
\hline $\begin{array}{l}\text { Incomplete outcome data } \\
\text { (attrition bias) } \\
\text { All outcomes }\end{array}$ & Unclear risk & Reasons for dropout were not reported. \\
\hline $\begin{array}{l}\text { Selective reporting (re- } \\
\text { porting bias) }\end{array}$ & High risk & Means and SDs from primary and secondary outcomes are not stated. \\
\hline Other bias & Low risk & No other biases identified. \\
\hline
\end{tabular}

\section{Schmidt 2018}

\begin{tabular}{ll}
\hline Methods & Two-arm, single-blinded, parallel-group RCT, with 6 weeks duration of treatment and 8-week follow-up \\
\hline Participants & Location: West Haven, USA, 1 site (Veterans Affairs Connecticut Healthcare System (VACHS)) \\
& Setting of recruitment and treatment: West Haven VACHS, a tertiary care Veterans Affairs facility \\
& Sample size: 25 \\
- Number randomised: 14 in VET CBT-T, 11 in audiological counselling \\
- Number completed: 11 in VET CBT-T, 9 in audiological counselling
\end{tabular}


Schmidt 2018 (Continued)

\section{Participant (baseline) characteristics:}

- Age: 61.3 (VET CBT-T), 66.4 (audiological counselling)

- Gender: 11 male in VET CBT-T, 9 males in audiological counselling

- Tinnitus duration: 22.9 years (intervention), 23.9 years (comparison)

Inclusion criteria: veterans with chronic ( $>6$ months) tinnitus, 18 years and older, must never had previously received psychological treatment for their tinnitus, have motivation to comply with treatment and able to commit to a 6-week course of treatment, follow-up and study participation by continuing to reside nearby

Exclusion criteria: those who scored 4 or lower on Tinnitus-Impact Screening Interview (TISI); any indication of psychosis on Structured Clinical Interview for Diagnosis, abbreviated - Interview/Non-patient (SCIDa-I/NP); scores of 19 or lower on Tinnitus Handicap Inventory (THI); 16 or lower on Tinnitus Reaction Questionnaire (TRQ); participants who were undergoing litigation or legal matters related to auditory disorders; potential participants with otherwise treatable tinnitus; history of psychotic disorders or dementia; psychotic symptoms (including, for example, delusions of reference, persecutory delusions, somatic delusions); having a recent (within 3 months) history of alcohol or drug abuse or dependence other than tobacco or caffeine; sudden or fluctuating hearing loss; tinnitus associated with otologic disease (e.g. Ménière's Disease) or other co-occurring diseases affecting vestibular dysfunction were excluded

Interventions

VET CBT-T: approximately weekly, group, tinnitus education plus cognitive behavioural therapy provided by a clinical psychologist. CBT included: included identification of individual responses and beliefs about tinnitus and hearing loss, re-conceptualisation of the tinnitus experience as one in which the patient has personal control, presentation of skills to modify cognitions and change behaviours, and reinforcement of skills via goals setting, homework and activities. Skills related to attention control, sleep hygiene, relaxation training are provided. Tinnitus education also included causes, treatments, current research, epidemiological information, basic anatomy of the ear and brain, and support resources.

Comparator: audiologist counselling. Approximately weekly, group-based tinnitus education provided by an audiologist over 6 weeks. Tinnitus education and skills related to attention control, sleep hygiene and relaxation training such as imagery techniques were provided. Tinnitus education also included information about: causes, treatments, current research, epidemiological information, basic anatomy of the ear and brain and support resources.

Use of additional interventions: none

\begin{tabular}{|c|c|c|}
\hline Outcomes & \multicolumn{2}{|c|}{$\begin{array}{l}\text { Primary outcome: Tinnitus Handicap Inventory (THI) } \\
\text { Secondary outcomes: Tinnitus Reaction Questionnaire (TRQ) } \\
\text { Measurement time points: pre, post and } 8 \text { weeks follow-up }\end{array}$} \\
\hline Funding sources & \multicolumn{2}{|c|}{ Study sponsor: VA Office of Research and Development } \\
\hline Declarations of interest & \multicolumn{2}{|l|}{ Not reported } \\
\hline Notes & \multicolumn{2}{|c|}{$\begin{array}{l}\text { Participants lost to follow-up: } 5 \text { ( } 3 \text { in intervention group, } 2 \text { in comparison group) out of total of } 25 \\
(20 \%) \text { dropped out }\end{array}$} \\
\hline \multicolumn{3}{|l|}{ Risk of bias } \\
\hline Bias & Authors' judgement & Support for judgement \\
\hline $\begin{array}{l}\text { Random sequence genera- } \\
\text { tion (selection bias) }\end{array}$ & Low risk & $\begin{array}{l}\text { Simple randomisation was conducted by drawing numbers from a hat with } \\
\text { even numbers indicating allocation to the intervention group (i.e. VET CBT- } \\
\text { T) and odd numbers indicating allocation to the control group (i.e. audiologi- }\end{array}$ \\
\hline
\end{tabular}


Schmidt 2018 (Continued)

cal counselling). This information is not reported but obtained from personal communication with the corresponding author.

\begin{tabular}{|c|c|c|}
\hline $\begin{array}{l}\text { Allocation concealment } \\
\text { (selection bias) }\end{array}$ & Unclear risk & Information not reported in the manuscript. \\
\hline $\begin{array}{l}\text { Blinding of participants } \\
\text { and personnel (perfor- } \\
\text { mance bias) }\end{array}$ & Unclear risk & $\begin{array}{l}\text { Although the study reports single blinding, no information is provided about } \\
\text { how this was achieved. }\end{array}$ \\
\hline All outcomes & & $\begin{array}{l}\text { Quote: "The study used a single-blind, randomised, parallel treatment (VET } \\
\text { CBT-T vs AC) concurrent design complemented by collection of qualitative da- } \\
\text { ta." }\end{array}$ \\
\hline
\end{tabular}

$\begin{array}{ll}\text { Blinding of outcome as- } & \text { High risk }\end{array}$

All outcomes

\begin{tabular}{ll}
\hline $\begin{array}{l}\text { Incomplete outcome data } \\
\text { (attrition bias) }\end{array}$ & Low risk \\
All outcoms for missing data reported and were similar across groups. There was \\
no statical analysis conducted due to the small sample size.
\end{tabular}

All outcomes

Quote: "Because of the small sample size, required assumptions for analysing parametric tests of linear effects were not met. Thus, only descriptive results and mean differences in scores on the THI and TRQ between these 3 assessment periods are presented."

\begin{tabular}{lll}
\hline $\begin{array}{l}\text { Selective reporting (re- } \\
\text { porting bias) }\end{array}$ & High risk & $\begin{array}{l}\text { The primary outcome was specified in the study protocol but not reported as } \\
\text { such in the publication. Additional outcomes not identified in the protocol } \\
\text { were added to the publication. }\end{array}$ \\
\hline Other bias & High risk & $\begin{array}{l}\text { Significant additional differences between the protocol and publication, e.g. } \\
\text { protocol includes a "no-intervention/standard care condition" but this is not } \\
\text { reported in the publication. Group } 2 \text { is described specifically as "tinnitus edu- } \\
\text { cation" in the protocol and "structured audiologist counselling" in the publica- } \\
\text { tion. The components of this comparator differ between the protocol and pub- } \\
\text { lication. }\end{array}$
\end{tabular}

Weise 2016

Methods Two-arm, non-blinded, parallel-group RCT, with 10 weeks of treatment and 12 months follow-up (intervention group only)

Participants Location: Germany

Setting of recruitment and treatment: participants were recruited from several health-related websites, public media and self-help groups for the study between April 2010 and December 2011. Both the intervention and comparator were conducted online.

\section{Sample size: 124}

- Number randomised: 62 in iCBT intervention, 62 in online discussion forum

- Number completed: 58 in iCBT, 61 in online discussion forum

\section{Participant (baseline) characteristics:}

- Age: 47.8 years in iCBT, 47.5 years in online discussion forum

- Gender: 74 females (37 in iCBT, 37 in online discussion forum), 50 males (25 in iCBT, 25 in online discussion forum)

- Tinnitus duration: 7.3 years in $\mathrm{ICBT}, 7.3$ years in online discussion forum 
Weise 2016 (Continued)

Inclusion criteria: 1) age at least 18 years; 2 ) subjective tinnitus (confirmed by an ear, nose, throat (ENT) specialist) with a duration of at least 6 months, which is present for most time during the day; 3 ) total score of 38 or higher on the Tinnitus Handicap Inventory (THI) (25) or of 13 or higher on the Mini-Tinnitus Questionnaire (Mini-TQ) (26); 4) no tinnitus-specific psychological tinnitus treatment within the last 2 years; 5) tinnitus as the primary problem and not only a consequence of a medical disease (e.g. Morbus Ménière), as determined by an ENT specialist; 5) access to a computer with an Internet connection; 6) sufficient reading and writing skills; 7) capacity of a minimum of 2 hours time per week for a period of 10 weeks to complete the treatment; 8) no severe medical or psychiatric condition; and 9) no acute suicidality.

Exclusion criteria: 1) tinnitus caused by any other general medical condition or otologic disease (e.g. active Ménière's disease); 2) clinical diagnosis of any severe mental disorder (especially a severe depressive disorder, suicidality, acute psychosis); 3) clinical diagnosis of dementia or another severe organic cerebral disorder; 4) clinical diagnosis of substance-related addiction/abuse

Interventions Therapists in the study worked with both groups of participants were either licensed CBT therapists ( $\mathrm{n}$ $=2$ ) or masters' level clinical psychologists $(n=2)$. All therapists had previously worked with tinnitus patients and received supervision throughout the course of the study.

ICBT: the 10-week guided Internet-based self-help programme used in the current trial was based on an existing, CBT self-help manual (Kaldo 2004a) but translated into German. Where necessary, the programme was adapted for use in Germany (e.g. typical sayings, names or culture-specific examples) and the text shortened where possible. An extra module on illness behaviours was also included.

The iCBT protocol comprised 12 mandatory and 6 optional text modules. Whereas the mandatory modules covered strategies to reduce tinnitus-related distress (e.g. relaxation, exposure to tinnitus or cognitive restructuring), the optional modules addressed problems potentially associated with tinnitus such as sleep, hearing or concentration problems. Patients were free to choose only those optional modules that were of special interest to them.

The programme explicitly states that the treatment does not target tinnitus loudness. Four days before the programme started, participants were given access to the online platform to get familiarised with the treatment rationale. Moreover, they were required to decide about optional modules with which they wanted to work. Participants were advised not to work on more than 2 to 3 new modules during a week. Participants made their choices for the treatment plan in an online form and sent it to their therapists who, in exchange, commented on the plan and gave additional advice. Each module was structured in the same way: theory and general information, exercises, worksheets and solutions for common problems. All treatment weeks started with a) downloading of the text modules or spoken instructions available as audio files, followed by b) reading the theoretical framework and c) conducting exercises in daily life. Once per week, patients could communicate with the therapist via a secured encrypted web page. Therapists were instructed to spend a maximum of 10 minutes/week per patient for email correspondence.

Comparator: internet-based discussion forum. A confidential, moderated, online discussion forum for 10 weeks acted as a control condition. Participants were invited to discuss tinnitus-related topics with other participants of the discussion forum group. Every week, the therapists posted a new discussion topic, which was related to tinnitus (e.g. representation of tinnitus in public media), but did not target strategies to improve tinnitus-related distress. However, if patients came up with treatment-related topics by themselves, they were free to discuss them. Therapists monitored the postings to assure their appropriateness.

Use of additional interventions: none reported

Measurement time points: pre and post intervention 
Weise 2016 (Continued)

Funding sources

The study was funded, in part, by a grant from the Swedish Research Council (HEAD Linnaeus Grant No. 349-2007-8654)

Declarations of interest

No conflicts of interest to declare

Notes

Participants lost to post-assessment: 5 (4 in iCBT, 1 in online discussion forum). 55/62 participants in iCBT group provided data at 12-months follow-up.

Adverse effects: reliable change index measures showed "reliable deterioration" on 3 participants on THI $(n=2)$ and Mini-TQ $(n=)$ at 12-months follow-up

Other: the comparator group did not complete follow-up measures, which were given to the iCBT group at 6- and 12-month follow-up

\section{Risk of bias}

\begin{tabular}{lll}
\hline Bias & Authors' judgement & Support for judgement \\
\hline $\begin{array}{l}\text { Random sequence genera- } \\
\text { tion (selection bias) }\end{array}$ & Low risk & $\begin{array}{l}\text { Used an online randomisation service (www.randomization.com) to generate } \\
\text { a randomisation list with no restrictions. }\end{array}$ \\
\hline $\begin{array}{l}\text { Allocation concealment } \\
\text { (selection bias) }\end{array}$ & Low risk & An independent researcher conducted the randomisation. \\
\hline $\begin{array}{l}\text { Blinding of participants } \\
\text { and personnel (perfor- } \\
\text { mance bias) } \\
\text { All outcomes }\end{array}$ & Unclear risk & This was an open-label study as per the protocol. \\
\hline
\end{tabular}

Blinding of outcome as- Unclear risk Information not reported in the manuscript.

sessment (detection bias)

All outcomes

\begin{tabular}{|c|c|c|}
\hline $\begin{array}{l}\text { Incomplete outcome data } \\
\text { (attrition bias) } \\
\text { All outcomes }\end{array}$ & Low risk & $\begin{array}{l}\text { Attrition and missing data reported with no significant differences between } \\
\text { groups. Multiple imputation was used, and the imputed and complete cases } \\
\text { analyses conducted. }\end{array}$ \\
\hline
\end{tabular}

\begin{tabular}{|c|c|c|}
\hline $\begin{array}{l}\text { Selective reporting (re- } \\
\text { porting bias) }\end{array}$ & High risk & $\begin{array}{l}\text { Numerous secondary outcome measures listed in the protocol were not re- } \\
\text { ported in methods or results, including outcomes of interest to the review. }\end{array}$ \\
\hline
\end{tabular}

\begin{tabular}{ll}
\hline Other bias $\quad$ Low risk $\quad$ The study appears to be free of other sources of bias. \\
\hline
\end{tabular}

\section{Westin 2011}

\begin{tabular}{ll}
\hline Methods & Three-arm, non-blinded, parallel-group RCT, with 10 weeks treatment and 16 months follow-up \\
\hline Participants & Location: Sweden \\
& Setting of recruitment and treatment: participants were recruited from 3 different audiology depart- \\
ments and via advertisements and articles in newspapers over the course of 17 months. All were reg- \\
istered as regular patients within the public healthcare system and diagnostic assessments and treat- \\
ments were provided within a hospital setting. The first intake in the study took place in August 2006 \\
and the last data were collected in June 2009.
\end{tabular}

\section{Sample size: 64}

- Number randomised: 22 to ACT, 20 to TRT, 22 to wait list control 


\section{Participant (baseline) characteristics:}

- Age: 53.5 years in ACT, 48.9 years in TRT, 49.6 years in wait list control, 50.9 overall

- Gender: 30 females (14 in ACT, 8 in TRT, 8 in wait list); 32 males (6 in ACT, 12 in TRT, 14 in wait list control)

- Tinnitus duration: 6.8 years in ACT, 9.2 years in TRT, 7.1 years in wait list control

Inclusion criteria: 1) potential participants had to have tinnitus as their primary problem; 2) to be at least 18 years old; 3 ) to have a score of at least 30 on the Tinnitus Handicap Inventory; 4) have had tinnitus for at least 6 months; 5) not to suffer from a severe psychiatric disorder; 6) not to have previously received a psychological or sound-generator treatment for tinnitus; 7 ) not be in need of immediate medical consultation; and 8) have hearing thresholds which would allow for the use of wearable sound generators (i.e. in severe hearing loss the sound stimulation may not be heard or need to be so loud that the person would have problems hearing conversations)

Exclusion criteria: not reported

Interventions
ACT: 5 female and 3 male therapists delivered the ACT treatment. Six therapists were masters programme students in clinical psychology who were receiving their clinical training, and 2 were clinical psychologists. Therapists were monitored through videotapes for treatment adherence and received weekly supervision by 2 licensed psychologists and psychotherapists who had extensive experience in ACT and supervision.

The ACT treatment was delivered in up to 10 weekly, 60- to 75-minute sessions in an individual format using a treatment manual developed according to the principles of Hayes 1999. Acceptance and Commitment Therapy for tinnitus included the following. Session 1: introduction to/assessment of tinnitus and tinnitus distress; treatment rationale; a brief introduction to mindfulness; and a mindfulness exercise. Session 2: creative hopelessness (contacting valued direction, evaluating earlier and current coping strategies related to tinnitus). Session 3: creative hopelessness (examining the costs of current strategies, introducing control as the problem and willingness as an alternative). Session 4: willingness and acceptance (including a mindfulness exercise in which the patient approaches tinnitus and associated reactions in a non-judgemental way). Session 5: values. Session 6: values, commitment and dealing with barriers (diffusion). Sessions 7 to 9: commitment and dealing with barriers depending on the patients' specific barriers the treatment has contained one or several of the following components: defusion, acceptance, behavioural hearing tactics, exposure to sound, problem solving and behaviour therapy related to insomnia (sleep restriction and stimulus control. Session 10: relapse prevention, repetition and preparation for possible difficult situations.

Comparator: tinnitus retraining therapy. The patients in the TRT condition received a single $2 \frac{1}{2}$ hour consultation with an ear, nose and throat physician and subsequently used wearable sound generators daily over 18 months following the principles outlined by Jastreboff 2004. The ear, nose and throat physician was a specialist in audiology, had extensive clinical experience of assessing and treating tinnitus patients, and experience with TRT. The consultation included: medical evaluation, taking the history of tinnitus, decreased sound tolerance and hearing loss, and assessing the category for treatment using the criteria presented by Jastreboff and Hazell (2004) in order to adjust treatment accordingly. The consultation included retraining counselling with education about the neurophysiological model of tinnitus. Patients were also given an introduction to sound therapy and instructions on how to wear and monitor their wearable sound generators. The instruction was to wear the devices throughout the waking hours. If this for any reason was impossible a minimum of 8 hours should be attempted, which may be divided into several shorter blocks of time. The intensity of the sound enrichment was set to the "mixing point", at which level partial suppression of the tinnitus sound begins to occur. Participants were given a 30-minute follow-up session over telephone following the same principles.

The sound generators were fitted bilaterally with an open fitting by an audiologist.

Comparator: wait list control. Participants received CBT either in an individual, self-help or group format after the 10-week waiting period.

Use of additional interventions: none reported

Primary outcome: Tinnitus Handicap Inventory (THI) 
Westin 2011 (Continued)

Secondary outcomes: Hospital Anxiety and Depression Scale-Anxiety (HADS-A) subscale, Hospital Anxiety and Depression-Depression subscale (HADS-D); Tinnitus Acceptance Questionnaire (TAQ); Insomnia Severity Index (ISI); The Quality of Life Inventory (QoLI); Clinical Global Impression-Improvement (CGI-I)

Measurement time points: pre-treatment and at 10 weeks (i.e. after the ACT intervention was completed), 6 and 18 months

Funding sources

The Medical Research Council of Southeast Sweden, and the Swedish Council for Working Life and Social Research. Other sources of funding were Starkey and GN Resound who both contributed by giving a discount on their Wearable Noise Generators. The role of the sponsors was strictly financial and they had no involvement in study design, collection, analysis and interpretation of data; writing of the report; or in the decision to submit the paper for publication.

Declarations of interest There are no financial, personal or other relationships that might lead to a conflict of interest.

Notes Participants lost to follow-up: 8 ( 1 in ACT, 6 in TRT, 1 in wait list control)

Adverse effects: deterioration as calculated by the reliable change index occurred in 3 participants (1 in ACT, 2 in TRT) at the 6 month follow-up

Other: wait list control group participants' data after the second measurement point was not included in the analyses

\section{Risk of bias}

\begin{tabular}{|c|c|c|}
\hline Bias & Authors' judgement & Support for judgement \\
\hline $\begin{array}{l}\text { Random sequence genera- } \\
\text { tion (selection bias) }\end{array}$ & Low risk & $\begin{array}{l}\text { Randomisation was performed using a computer-generated list (www.ran- } \\
\text { dom.org) with no restrictions. }\end{array}$ \\
\hline $\begin{array}{l}\text { Allocation concealment } \\
\text { (selection bias) }\end{array}$ & High risk & $\begin{array}{l}\text { The trial co-ordinator handled randomisation being blind to pre-assessment } \\
\text { data, but using no concealment in the allocation to study condition. }\end{array}$ \\
\hline $\begin{array}{l}\text { Blinding of participants } \\
\text { and personnel (perfor- } \\
\text { mance bias) } \\
\text { All outcomes }\end{array}$ & Unclear risk & $\begin{array}{l}\text { There was no blinding of participants or personnel, but there was insufficient } \\
\text { information available to permit a judgement of low risk or high risk. }\end{array}$ \\
\hline $\begin{array}{l}\text { Blinding of outcome as- } \\
\text { sessment (detection bias) } \\
\text { All outcomes }\end{array}$ & Unclear risk & $\begin{array}{l}\text { The outcome measures were self-reported/administered questionnaires, but } \\
\text { there was insufficient information available to permit a judgement of low risk } \\
\text { or high risk. }\end{array}$ \\
\hline $\begin{array}{l}\text { Incomplete outcome data } \\
\text { (attrition bias) } \\
\text { All outcomes }\end{array}$ & Unclear risk & $\begin{array}{l}\text { Some participants underwent treatment but did not complete outcome as- } \\
\text { sessment. Missing data assumed to be missing at random - mixed modelling. } \\
\text { Some participants completed outcomes without starting treatment. }\end{array}$ \\
\hline $\begin{array}{l}\text { Selective reporting (re- } \\
\text { porting bias) }\end{array}$ & Low risk & $\begin{array}{l}\text { Protocol not available but all the outcomes mentioned in the methods were } \\
\text { reported in the results. }\end{array}$ \\
\hline Other bias & Low risk & No other sources of bias identified. \\
\hline
\end{tabular}

Zhong 2014

\begin{tabular}{ll}
\hline Methods & Two-arm, parallel-group RCT at 2, 6 and 12 months follow-up \\
\hline Participants & Location: Pingxiang City, Jiangxi Province, China
\end{tabular}


Setting of recruitment and treatment: participants were recruited from the Pingxiang City Peoples' Hospital Otolaryngology Clinic. (The setting for the treatment was not reported).

Sample size: 157

- Number randomised: 89 in CBT, 68 in masking

- Number completed: not reported

Participant (baseline) characteristics:

- Age: 42.8 years in the CBT group, 42.6 in the masking group

- Gender: 55 females (25 in the CBT group, 30 in masking condition), 102 males (64 males in CBT, 38 in masking)

- Tinnitus duration: 2.6 years in CBT group, 2.4 years in masking group

Inclusion criteria: Quote: "Patients with continuous tinnitus or tinnitus symptoms at night, accompanied by negative emotions such as anxiety and irritation."

Exclusion criteria: 1)local or systemic acute inflammation; 2) history of local or systemic tumours; 3 ) metabolic disease; 4) immune-related disease; 5) severe diseases of internal medicine; 6) otologic diseases

Interventions

CBT: the intervention included: cognitive restructuring, problem solving and training to shift the attention away from tinnitus. Treatment was tailored to individual needs. The frequency, duration and providers of the CBT intervention were not reported.

Comparator: masking. Participants chose (with or without clinician advice) on the type of noise to use as masking sound. It was then given at the lowest volume of noise able to mask tinnitus. It was administered 1 to 3 times a day, for 15 to 20 minutes each time. If there was any change in tinnitus, the noise used to mask the tinnitus was re-selected and adjusted.

Use of additional interventions: sound therapy composed of: advising the use of MP3 as a tinnitus masker to treat tinnitus using 2 kinds of superimposing sounds: 1) "low" volume masking sound (volume being the same as threshold to cover the ear sounds); and 2) "high" volume music background (can also be replaced with watching TV or listening to music). Participants were instructed to focus on music (or listen to TV sounds) during treatment in order to decrease the amount of attention given to tinnitus.

Outcomes

Primary outcome: Tinnitus Handicap Inventory (THI) grouped into 4 categories. First quartile/Category 1: "no handicap", THI score 0 to 16. Second quartile/Category 2: "mild handicap", THI score 18 to 36. Third quartile/Category 3: "moderate handicap" THI score 38 to 56. Fourth quartile/Category 4: "severe handicap", THI score 58 to 100.

Secondary outcomes: not reported

\begin{tabular}{ll}
\hline Funding sources & Not reported \\
\hline Declarations of interest & Not reported \\
\hline Notes & Participants lost to follow-up: unknown \\
& $\begin{array}{l}\text { Adverse effects: not reported } \\
\text { Means, standard deviations and the numbers of participants at each time point requested from the cor- } \\
\text { responding author }\end{array}$
\end{tabular}

\section{Risk of bias}

\begin{tabular}{lll}
\hline Bias & Authors' judgement & Support for judgement \\
\hline $\begin{array}{l}\text { Random sequence genera- } \\
\text { tion (selection bias) }\end{array}$ & Unclear risk & $\begin{array}{l}\text { Participants were randomised to the intervention and control groups but the } \\
\text { method used was not reported. }\end{array}$ \\
\hline
\end{tabular}


Zhong 2014 (Continued)

$\begin{aligned} & \text { Allocation concealment } \\ & \text { (selection bias) }\end{aligned} \quad$ Unclear risk Information not reported in the article.

Blinding of participants Unclear risk Information not reported in the article.
and personnel (perfor-
mance bias)
All outcomes

\begin{tabular}{ll}
\hline Blinding of outcome as- & Unclear risk
\end{tabular} Information not reported in the article.

sessment (detection bias)

All outcomes

\begin{tabular}{lll}
\hline $\begin{array}{l}\text { Incomplete outcome data } \\
\text { (attrition bias) } \\
\text { All outcomes }\end{array}$ & Low risk & All participants completed the study. \\
\hline $\begin{array}{l}\text { Selective reporting (re- } \\
\text { porting bias) }\end{array}$ & Unclear risk & Insufficient information to judge 'high' or 'low' risk. \\
\hline Other bias & Low risk & No other sources of bias identified. \\
\hline
\end{tabular}

$\mathrm{ACl}$ : attention control and imagery training

ACT: acceptance and commitment therapy

ATQ: Automatic Thoughts Questionnaire

BDI: Beck Depression Inventory

CBT: cognitive behavioural therapy

CBT-T: cognitive behavioural therapy plus tinnitus education

CCEI: Crown-Crisp Experiential Index

CET: coping effectiveness training

CFQ: Cognitive Failures Questionnaires

CR: cognitive restructuring

ENT: ear, nose and throat

FTQ: Fear of Tinnitus Questionnaire

GAD-7: Generalized Anxiety Disorder-7

GCT: group cognitive therapy

GP: general practitioner

HADS: Hospital Anxiety and Depression Scale

HADS-A: Hospital Anxiety and Depression Scale - Anxiety subscale

HADS-D: Hospital Anxiety and Depression Scale - Depression subscale

HHIA-S: Hearing Handicap Inventory for Adults Screening version

HQ: Hyperacusis Questionnaire

HRD/HRSD: Hamilton Rating Scale for Depression

HUI: Health Utilities Index

iCBT: Internet-based CBT

ISI: Insomnia Severity Index

IWDA: Interference with Daily Activities

MBCT: Mindfulness Based Cognitive Therapy

MSPQ: Modified Somatic Perception Questionnaire

NHS: National Health Service (UK)

PHQ-9: Patient Health Questionnaire-9

QIPA: Tinnitus Psychological Impact Questionnaire

QoLI: Quality of Life Inventory

$\mathrm{RCT}$ : randomised controlled trial

RT: relaxation therapy

SD: standard deviation

STAI: State Trait Anxiety Inventory

SWLS: Satisfaction with Life Scale

TAQ: Tinnitus Acceptance Questionnaire

TCQ: Tinnitus Cognitions Questionnaire 
TCS: Tinnitus Catastrophizing Scale

TCSQ: Tinnitus Coping Strategies Questionnaire

TEQ: Tinnitus Effects Questionnaire

TFI: Tinnitus Functional Index

THI-S: Tinnitus Handicap Inventory

THI-S: Tinnitus Handicap Inventory screening version

THQ: Tinnitus Handicap Questionnaire

TKQ: Tinnitus Knowledge Questionnaire

TQ: Tinnitus Questionnaire

TRQ: Tinnitus Reaction Questionnaire

TRT: tinnitus retraining therapy

VET: veterans

WHO QoL: World Health Organization Quality of Life

Characteristics of excluded studies [ordered by study ID]

Study Reason for exclusion

\section{Delb 2002}

Henry 2017
ALLOCATION: not randomised; arbitrary division of participants into groups

ALLOCATION: randomised

PARTICIPANTS: 300 veterans with tinnitus

INTERVENTIONS: not CBT. Although the intervention contained CBT, the majority of treatment, and for most patients, it was not and they did not receive CBT.
ALLOCATION: randomised

PARTICIPANTS: people at least 18 years of age with tinnitus of at least 3 months duration

INTERVENTIONS: Internet-based CBT compared with group CBT

COMPARATOR: no appropriate comparator group for this review

PARTICIPANTS: adults with tinnitus aged between 18 and 60 years

INTERVENTION: not CBT

Kröner-Herwig 1995

Kröner-Herwig 1999
ALLOCATION: initially allocation was done by throwing dice, but then some participants had to be re-assigned to different treatments for scheduling reasons (personal communication)

ALLOCATION: this study used the data obtained by Kröner-Herwig 1995 in which allocation was initially done by throwing dice, but then some participants had to be re-assigned to different treatments for scheduling reasons (personal communication)

Kröner-Herwig 2003

ALLOCATION: secondary analysis of data from Zachriat 2004, which was excluded because of nonrandomised allocation of participants. See Zachriat 2004 for details.

Kröner-Herwig 2006

ALLOCATION: secondary analysis of data from Zachriat 2004, which was excluded because of nonrandomised allocation of participants. See Zachriat 2004 for details.

Li 2019 ALLOCATION: randomised

PARTICIPANTS: adults with chronic subjective tinnitus

INTERVENTIONS: not CBT (CBT was combined with masking therapy and sound treatment) 


\begin{tabular}{ll}
\hline Study & Reason for exclusion \\
\hline Luyten 2019 & $\begin{array}{l}\text { ALLOCATION: not randomised ("The allocation sequence will be determined by the date the pa- } \\
\text { tients are referred to the study.") }\end{array}$ \\
\hline
\end{tabular}

Malouff $2010 \quad$ ALLOCATION: randomised

PARTICIPANTS: adults with tinnitus (no exclusion criteria applied)

INTERVENTIONS: not CBT. The intervention used was bibliotherapy (without therapist contact).

Rief 2005
PARTICIPNTS: adults suffering from tinnitus
INTERVENTIONS: not CBT. Although the intervention contains elements of CBT for tinnitus, it is a
"psychophysiologically oriented intervention" (i.e. biofeedback).

Scott $1985 \quad$ ALLOCATION: randomised

PARTICIPANTS: adults with tinnitus

INTERVENTION: not CBT (although the interventions included some elements found in CBT)

\begin{tabular}{|c|c|}
\hline \multirow[t]{2}{*}{ Tan 2018} & $\begin{array}{l}\text { ALLOCATION: unclear. Attempts made to obtain further information on allocation procedure. } \\
\text { PARTICIPANTS: adult patients with tinnitus }\end{array}$ \\
\hline & $\begin{array}{l}\text { INTERVENTION: not CBT. CBT combined with other interventions. "Experimental group received } \\
\text { conventional nursing as well as rehabilitation exercise and cognitive-behavioral intervention." }\end{array}$ \\
\hline
\end{tabular}

Tavakoli 2019 ALLOCATION: not randomised. Described as a "semi-experimental" study. Also, "The exclusion criteria were: unwillingness to continue with participation; attending meetings on an irregular basis; and not doing home exercises given to them."

Tucker 2013

ALLOCATION: unclear. Communications with the author and research assistant who allocated participants to treatment groups could not confirm how participants were actually allocated to groups.

Weise $2008 \quad$ ALLOCATION: randomised

PARTICIPANTS: adults with tinnitus for a minimum of 6 months

INTERVENTIONS: not CBT. Although the interventions included some elements of CBT, we concluded that the intervention was predominantly biofeedback.

\section{Zachriat 2004}

ALLOCATION: not randomised allocation of participants. Participants were grouped according to gender, age and tinnitus-related disability and were randomly assigned to the treatment conditions by throwing dice. However, "practical reasons lead to the assignment of different numbers of patients to the groups....while 31 were assigned to HabituationTreatment and 29 to Tinnitus Coping Training, only 23 were assigned to Educational control group because this number seemed sufficient to test the hypothesis of a lower success rate in this group."

\section{Zenner 2013}

ALLOCATION: not randomised. All patients at 4 treatment centres were allocated to CBT groups while all patients at a fifth treatment centre were allocated to the wait list control condition. 


\section{Study Reason for exclusion}

INTERVENTION: not CBT. Intervention described as "resilience training", which suggests it is neither CBT nor "therapy". Attempts made to contact the authors for further information but no response received.

CBT: cognitive behavioural therapy

Characteristics of ongoing studies [ordered by study ID]

\section{NCT03022084}

\section{Trial name or title}

\begin{tabular}{|c|c|}
\hline Methods & 2-arm randomised controlled (parallel-group) trial \\
\hline Participants & $\begin{array}{l}\text { Inclusion criteria: } \\
\text { - Age } \geq 18 \text { years } \\
\text { - Primary and persistent tinnitus ( } 6 \text { months or longer in duration) } \\
\text { - Tonal tinnitus } \\
\text { - Dominant tinnitus frequency measured between } 0.2 \mathrm{kHz} \text { and } 10 \mathrm{kHz} \\
\text { - Tinnitus Questionnaire score } \\
\text { - No current participation in other tinnitus therapy programme } \\
\text { - Willing and able to listen to the acoustic prescription for } 4 \text { to } 6 \text { hours daily during the trial } \\
\text { - Able to pass the Tone Audibility Assessment with factor of } 1.1 \\
\text { Exclusion criteria: } \\
\text { - Secondary/somatic tinnitus due to a suspected underlying disease } \\
\text { - Atonal, pulsatile, intermittent or occasional tinnitus } \\
\text { - Any hearing threshold }>70 \mathrm{~dB} \text { HL from } 0.25 \mathrm{kHz} \text { to } 8 \mathrm{kHz} \text {, unless participant passes the Tone Au- } \\
\text { dibility Assessment screening with a factor of } 1.1 \\
\text { - Any health or other problems that may prevent the person from completing the study procedures } \\
\text { as determined by investigator } \\
\text { - Participant reports current suicidal ideation and/or homicidal ideation } \\
\text { - Use of medication which may trigger tinnitus (e.g. quinine derivatives, aminoglycoside antibi- } \\
\text { otics, daily high-dose non-steroidal anti-inflammatory drugs } \geq 1000 \text { mg, salicylates (when not pre- } \\
\text { - scribed as a low dose for cardiac health), loop diuretics and chemotherapy agents like cisplatin) } \\
\text { - Conductive hearing loss } \\
\text { - Visible congenital or traumatic deformity of the ear } \\
\text { - History of active drainage from the ear within the past } 90 \text { days } \\
\text { - History of sudden or rapidly progressive hearing loss within the past } 90 \text { days } \\
\text { - Inability to read and respond appropriately to instructions that appear on the computer screen } \\
\text { and/or to perform all of the procedures } \\
\text { - Previous use of Desyncra for Tinnitus Therapy System }\end{array}$ \\
\hline
\end{tabular}

Intervention: cognitive behavioural therapy

Active comparator: this group will use the sound therapy device Desyncra for Tinnitus Therapy System

Primary outcome: change from baseline Tinnitus Questionnaire score (time frame: 24 weeks)

Secondary outcome: none listed

Starting date January 2017

Cognitive behavioural therapy for tinnitus (Review)

Copyright $\odot 2020$ The Cochrane Collaboration. Published by John Wiley \& Sons, Ltd. 
NCT03022084 (Continued)

Contact information
Sarah Theodoroff, Research Investigator

VA Portland Health Care System

National Center for Rehabilitative Auditory Research

Portland, Oregon, United States, 97239

Notes ClinicalTrials.gov Identifier: NCT03022084

\section{NCT03386123}

Trial name or title

Methods

Participants
'A comparison of cognitive behaviour therapy for insomnia (CBTi) and usual audiological rehabilitation in the management of tinnitus related insomnia'

Three-arm randomised controlled (parallel-group) trial

Planned sample size: 111

Inclusion criteria:

- Aged 18 to 70

- At least moderately distressing tinnitus (above a set minimum score on the Mini TQ) for at least 6 months and problematic insomnia (above a set minimum score on the Insomnia Severity Index (ISI) and for tinnitus-related insomnia to be identified in clinical interview) as result of tinnitus, for at least 3 months

- Patient wishes to work on improving sleep

- Sufficient understanding of English and sufficient hearing ability to take part in group discussions and to complete questionnaires

- Patient has had tinnitus assessed by a doctor and an audiological specialist

- Willing and able to provide written consent

- Able to regularly attend clinic in London, United Kingdom

Exclusion criteria:

- Organic sleep disorders present (e.g. obstructive sleep apnoea, delayed phase sleep, etc.) assessed with a subscale of the hospital sleep unit's sleep disorder and snoring proforma

- Currently pregnant, planning pregnancy or breastfeeding

- Alcohol or drug dependent

- Currently suffering with severe mental illness (psychosis, severe anxiety or mood disorder)

- Reports active risk to themselves or others

- Still undergoing medical investigations into sleep and/or tinnitus
Intervention: cognitive behavioural therapy for insomnia (CBTi). A standard CBTi programme for the treatment of primary insomnia, with $6 \times 2$-hour group sessions over 8 weeks. There will be minor adaptations for tinnitus, including making specific reference to tinnitus and psycho-education about tinnitus. Every session concludes with provision of a homework task and a sleep diary to complete over the next week. The CBTi course will be supported by providing participants with a CD with some relaxation exercises and a booklet that covers the information given in the session. CBTi includes: sleep restriction, stimulus control, sleep hygiene, relaxation training, paradoxical intention, cognitive therapy: targeting unhelpful beliefs about sleep and worry, behavioural experiments: testing unhelpful beliefs and adjusting sleep related behaviour. Intervention: behavioural: cognitive behavioural therapy for insomnia

Active comparator: standard audiological care (SAC). A group intervention that fits with reported audiological treatment of people with tinnitus and significant sleep impairment. This involves psycho-education about tinnitus, habituation, sleep and sleep hygiene. Relaxation will be advised and 
information provided. A bedside sound generator, as used in routine clinical practice, will be provided. Information will be based on standard advice given by hearing therapists/audiologists and will not include specific psychological techniques which are not part of SAC. The group will be generally supportive. SAC tends not to involve repeated meetings; after the initial session, there will be one follow-up session 8 weeks later. Follow-up will allow for question and answer, and reports on what has been useful. Both sessions will last for 2 hours. Intervention: Behavioural: standard audiological care.

Placebo comparator: sleep support group (SSG) Participants will meet in a group, which will offer equivalent contact with therapists and a supportive group milieu as CBTi. It will focus on the potential benefits of a supportive group and will not include specific advice. Participants will complete 2-week sleep diaries as baseline and outcome measures at the 4 time points, which will be checked within the session to ensure that participants know how to complete them correctly. The SSG will meet in a group for 6 sessions, over 8 weeks, each of 2 hours duration. Intervention: behavioural: sleep support group.
Outcomes

Cognitive behavioural therapy for tinnitus (Review)

Copyright $\odot 2020$ The Cochrane Collaboration. Published by John Wiley \& Sons, Ltd.

\section{Primary outcomes:}

\section{Secondary outcomes:} and at 1- and 6-month follow-ups) and at 1 and 6 month follow-ups) follow-ups) and at 1 - and 6-month follow-ups)
1. Change in Insomnia Severity Index (ISI) score (time frame: at first and last treatment sessions and at 1 - and 6-month follow-ups), 7-item questionnaire, each item rated 0 to 4 . Total scores range from 0 to 28 with higher scores indicating more severity.

2. Change in the amount of sleep obtained (time frame: a 2-week sleep diary will be kept 2 weeks prior to the first and last treatment sessions and to the 1- and 6-month follow-ups). Average of all of the following metrics obtained from 2 weeks of sleep diary measure: sleep onset latency lower score indicates better sleep; wake time after sleep onset - lower score indicate better sleep; number of night-time awakenings - lower scores indicate better sleep; total sleep time - higher scores indicate better sleep; time in bed - included only so that sleep efficiency can be calculated: sleep efficiency (total sleep time/time in bed x 100) - higher scores indicate better sleep. Diary measures for sleep quality $(0=$ worst possible to $10=$ best possible), tinnitus annoyance $(0=$ not at all to $10=$ extremely), refreshed at waking $(0=$ not at all to $10=$ very refreshed $)$ and quality of day time functioning $(0=$ very poor to $10=$ very good $)$.

1. Change in Pittsburgh Sleep Quality Index score (time frame: at first and last treatment sessions

2. Change in Dysfunctional Beliefs and Attitudes About Sleep Questionnaire - abbreviated version (DBAS-16) score (time frame: at first and last treatment sessions and at 1- and 6-month follow-ups)

3. Change in Tinnitus Catastrophizing Scale score (time frame: at first and last treatment sessions and at 1 - and 6-month follow-ups), 13-item questionnaire scored on a 0 to 4 scale

4. Mini Tinnitus Questionnaire score (time frame: screening). Construct: tinnitus complaint 12-item questionnaire scored on a 0 to 2 scale.

5. Change in the Tinnitus Questionnaire score (time frame: at first and last treatment sessions and at 1-and 6-month follow-ups). The reliable change criterion is 11.08.

6. Change in subjective measure of tinnitus loudness using a visual analogue scale (time frame: at first and last treatment sessions and at 1- and 6-month follow-ups)

7. Change in Clinical Outcome in Routine Evaluation - Outcome Measure score (time frame: at first and last treatment sessions and at 1- and 6-month follow-ups)

8. Change in Patient Health Questionnaire-9 score (time frame: at first and last treatment sessions

9. Change in Generalized Anxiety Disorder Assessment-7 score (time frame: at first and last treatment sessions and at 1- and 6-month follow-ups)

10.Change in EuroQOL score (time frame: at first and last treatment sessions and at 1- and 6-month

11.Change in Work and Social Adjustment Scale score (time frame: at first and last treatment sessions

12.Satisfaction and feedback (time frame: at the end of treatment and at 6-month follow-up point). Questionnaire asking individuals to rate the following on a 0 to 10 scale (from not at all to extremely). How useful was treatment? How relevant was treatment? How acceptable was treatment? 
NCT03386123 (Continued)

They are also asked to provide qualitative feedback in the form of comments in response to questions about what changes they have noticed, what aspects of treatment were most and least useful, what they would have liked to be different and any other comments.

\begin{tabular}{ll}
\hline Starting date & December 2017 \\
\hline Contact information & Laurence McKenna, PhD, laurence.mckenna@nhs.net \\
& Elizabeth Marks, D.Clin.Psych, e.marks@nhs.net \\
\hline Notes & Planned completion date: 30 June 2019 \\
& ClinicalTrials.gov identifier: NCT03386123 \\
\hline
\end{tabular}

\section{NCT04004260}

Trial name or title

'Cognitive Behavior Therapy Based Self-help Delivered Via the Internet for Tinnitus Sufferers: Efficacy Trial in the U.S. Population'

Methods 2-arm randomised controlled (parallel-group) trial

Participants

Inclusion criteria:

1. aged 18 years and older living in the State of Texas, USA;

2. the ability to read and type in English or Spanish;

3. no barriers to using a computer (e.g. no significant fine motor control or visual problems);

4. Internet and email access and the ability to use it;

5. commitment to completing the programme;

6. completion of the online screening and outcome questionnaires;

7. agreeing to participate in either group and be randomised to one of these groups;

8. understanding and working towards the end goal of reducing the impact and distress of tinnitus, although the strength of the tinnitus may remain the same;

9. be available for 12 months after starting the study to complete a 1-year follow-up questionnaire; 10.suffering with experiencing tinnitus for a minimum period of 3 months; and

11.tinnitus outcome measure scores indicating the need for tinnitus care ( 25 or above on the Tinnitus Functional Index (TFI)).

Exclusion criteria:

1. reporting any major medical or psychiatric conditions;

2. reporting pulsatile, objective or unilateral tinnitus, which has not been investigated medically;

3. tinnitus as a consequence of a medical disorder, still under investigation; and

4. undergoing any other tinnitus therapy while participating in this study.

Interventions

Intervention: the intervention offered is a guided Internet-based CBT intervention. The intervention is similar to a self-help programme, providing an opportunity to learn about new ways of coping with tinnitus during everyday life. It is a 8-week long e-learning intervention, with new modules introduced weekly and assignments are given to practice the techniques learned. Intervention: Behavioural: Internet-based cognitive behavioural therapy

Wait list control: the weekly check-in control group will be monitored weekly by means of the Tinnitus Handicap Inventory-Screening version (THI-S) and the Tinnitus Qualities Questionnaire (TQQ). Once the experimental group completes the iCBT intervention, the control group undertake the same iCBT intervention. 
NCT04004260 (Continued)

Outcomes
Primary outcome: change in Tinnitus Functional Index (TFI) (time frame: 8 weeks (baseline; 2 months - last treatment session)). Total possible score ranges from 0 to 100 (scores $>25$ indicate that tinnitus is a significant problem).

Secondary outcomes: change in Tinnitus and Hearing Survey (THS) (time frame: 8 weeks (baseline; 2 months - last treatment session)). The total THS score can range from 0 to 40 (sections $A$ and $B$ range 0 to 16 and section $C$ range from 0 to 8 ).

Change in Tinnitus Cognition Questionnaire (TCQ) (time frame: 8 weeks (baseline; 2 months - last treatment session)). The total score of the TCQ has a potential range from 0 to 104 . A high score represents a greater tendency to engage in negative cognitions in response to tinnitus and low engagement in positive cognitions.

Change in Patient Health Questionnaire (PHQ-9) (time frame: 8 weeks (baseline; 2 months - last treatment session)). The total score can range from 0 to 27 with higher scores indicating more severe depression.

Change in Generalized Anxiety Disorder (GAD-7) (time frame: 8 weeks (baseline; 2 months - last treatment session)). The total score can range from 0 to 21 with higher scores indicating more severe anxiety.

Change in Insomnia Severity Index (ISI) (time frame: 8 weeks (baseline; 2 months - last treatment session)). The total score can range from 0 to 28 with higher scores indicating more severe insomnia.

Change in EuroQol EQ-5D-5L (time frame: 8 weeks (baseline; 2 months - last treatment session)). The total score can range from 0 to 28 with higher scores indicating more severe insomnia.

\begin{tabular}{ll}
\hline Starting date & 1 February 2020 \\
\hline Contact information & Emily Born +14098802272 , eborn@lamar.edu \\
& Srinivas Palanki, PhD +14098807848, spalanki@lamar.edu \\
\hline Notes & $\begin{array}{l}\text { Estimated completion date: } 31 \text { August } 2021 \text { (final data collection date for primary outcome mea- } \\
\text { sure) } \\
\text { ClinicalTrials.gov Identifier: NCT04004260 }\end{array}$ \\
\hline
\end{tabular}

\section{NTR6415}

Trial name or title 'Bold exposure or safe masking? A fear-conditioning approach to chronic tinnitus suffering; a randomised controlled trial'

\begin{tabular}{ll}
\hline Methods & 2-arm randomised controlled (parallel-group) trial \\
\hline Participants & $\begin{array}{l}\text { Adults aged } 18 \text { or more with tinnitus and scores on the Tinnitus Questionnaire of TQ > 30; no previ- } \\
\text { ous masking or exposure therapy of minimally } 5 \text { years before inclusion }\end{array}$ \\
& $\begin{array}{l}\text { Exclusion criteria: hearing loss of more than } 40 \mathrm{~dB} \text { in either/both ears; limited reading and writing } \\
\text { skills in Dutch language }\end{array}$ \\
\hline
\end{tabular}

Interventions Intervention: exposure therapy for tinnitus

Active comparator: masking therapy for tinnitus

Outcomes

Primary outcomes: tinnitus disability as measured by: Tinnitus Handicap Inventory and Tinnitus Functional Index; tinnitus severity as measured by Tinnitus Questionnaire (TQ); and quality of life as measured by the Health Utilities Index and SF-36 
Secondary outcomes: tinnitus fear as measured by the Fear of Tinnitus Questionnaire (FTQ); catastrophic thoughts: Tinnitus Catastrophising Scale (TCS); tinnitus variability; threat expectancies; negative emotional status; avoidance behaviour as measured by the Inventory of Tinnitus Avoidance Behaviours (ITAB); daily diaries (including 3 weeks pre- and post intervention); tinnitus loudness and maskability questionnaire (LMI); and tinnitus-related fear-responding and tinnitus intensity

\begin{tabular}{ll}
\hline Starting date & 22 May 2017 \\
\hline Contact information & Dr. Rilana Cima \\
& r.cima@maastrichtuniversity.nl \\
& P.O. Box 616, Dept. of Clinical Psychological Science \\
& Maastricht University \\
& 6200 MD Maastricht \\
& The Netherlands \\
\hline Notes & Netherlands Trial Registry identifier: NTR6415 \\
\hline
\end{tabular}

\section{SLCTR/2018/005}

Trial name or title

'Effectiveness of modified cognitive behavioral therapy (CBT) in reducing distress for patients with tinnitus who are receiving treatment at 2 tertiary care hospitals in Sri Lanka, a randomised controlled trial'

\begin{tabular}{|c|c|}
\hline Methods & 2-arm, double-blind randomised controlled (parallel-group) trial \\
\hline \multirow[t]{7}{*}{ Participants } & $\begin{array}{l}\text { People over the age of } 18 \text { years with unilateral or bilateral tinnitus lasting for at least } 6 \text { months at } \\
\text { the commencement of the study }\end{array}$ \\
\hline & Exclusion criteria: \\
\hline & 1. Inability to converse in the Sinhala language \\
\hline & $\begin{array}{l}\text { 2. Participants who have not attained a basic educational level, i.e. who are unable to complete the } \\
\text { questionnaires alone }\end{array}$ \\
\hline & 3. Individuals who have had previously received psychological treatment for their tinnitus \\
\hline & 4. Participants with otherwise treatable tinnitus \\
\hline & 5. Participants with a history of psychotic disorders or dementia \\
\hline
\end{tabular}

Interventions

Intervention: patients assigned to the experimental group will receive 4 individual sessions of cognitive behavioural therapy (CBT) over 4 weeks, with each session lasting 45 minutes. The CBT will follow a structured manual which will comprise of these components:

1. Brief focused assessment

2. Education

3. Relaxation

4. Activity scheduling

5. Cognitive restructuring

6. Mindfulness

CBT sessions will be conducted at a mutually convenient time at the hospital premises by audiologists and psychiatrists trained in CBT. In order to ensure all the participating therapists are following the manual, therapists will have regular group supervision during the study.

Active comparator: the control group will receive standard care, which involves audiological measurement and brief education 
SLCTR/2018/005 (Continued)

Outcomes
Primary outcome: severity and handicap level of tinnitus using the Tinnitus Handicap Inventory (at baseline, 4 weeks after initiation and 24 weeks after initiation of the intervention)

Secondary outcome: potential non-psychotic or psychological problems using General Health Questionnaire - 12 (at baseline and 6 months after initiation of the intervention)

\begin{tabular}{ll}
\hline Starting date & February 2018 \\
\hline Contact information & Dr. Asiri Rodrigo \\
& Consultant Psychiatrist \\
& Department of Psychiatry, Faculty of Medicine, University of Kelaniya, Ragama, Sri Lanka \\
& Tel: 0112961115 \\
& Mob: 0777865791 \\
& Fax: 0112958837 \\
& asirir2000@yahoo.com \\
\hline Notes & Sri Lankan Clinical Trial Registry identifier: SLCTR/2018/005 \\
\hline
\end{tabular}

CBT: cognitive behavioural therapy

CBTi: cognitive behavioural therapy for insomnia

DATA AND ANALYSES

\section{Comparison 1. CBT versus no intervention/waiting list control}

\begin{tabular}{|c|c|c|c|c|}
\hline Outcome or subgroup title & No. of studies & $\begin{array}{l}\text { No. of partici- } \\
\text { pants }\end{array}$ & Statistical method & Effect size \\
\hline $\begin{array}{l}1 \text { Impact of tinnitus on quality of life } \\
\text { at end of treatment }\end{array}$ & 10 & 537 & $\begin{array}{l}\text { Std. Mean Difference (IV, Ran- } \\
\text { dom, } 95 \% \mathrm{Cl} \text { ) }\end{array}$ & $-0.56[-0.83,-0.30]$ \\
\hline $\begin{array}{l}2 \text { Serious adverse effects at end of } \\
\text { treatment }\end{array}$ & 7 & 447 & $\begin{array}{l}\text { Risk Ratio (IV, Random, 95\% } \\
\text { CI) }\end{array}$ & $3.00[0.13,69.87]$ \\
\hline 3 Depression at end of treatment & 8 & 502 & $\begin{array}{l}\text { Std. Mean Difference (IV, Ran- } \\
\text { dom, } 95 \% \mathrm{Cl} \text { ) }\end{array}$ & $-0.34[-0.60,-0.08]$ \\
\hline 4 Anxiety at end of treatment & 6 & 429 & $\begin{array}{l}\text { Std. Mean Difference (IV, Ran- } \\
\text { dom, } 95 \% \mathrm{Cl} \text { ) }\end{array}$ & $-0.45[-0.82,-0.09]$ \\
\hline $\begin{array}{l}5 \text { Health-related quality of life at } \\
\text { end of treatment }\end{array}$ & 2 & 179 & $\begin{array}{l}\text { Std. Mean Difference (IV, Ran- } \\
\text { dom, } 95 \% \mathrm{CI} \text { ) }\end{array}$ & $-0.38[-0.67,-0.08]$ \\
\hline $\begin{array}{l}6 \text { Negatively biased interpretations } \\
\text { of tinnitus at end of treatment }\end{array}$ & 2 & 84 & $\begin{array}{l}\text { Std. Mean Difference (IV, Ran- } \\
\text { dom, } 95 \% \mathrm{Cl} \text { ) }\end{array}$ & $-0.40[-1.25,0.45]$ \\
\hline $\begin{array}{l}7 \text { Subgroup analysis (random-ef- } \\
\text { fects model): type of therapy - im- } \\
\text { pact of tinnitus on quality of life at } \\
\text { end of treatment }\end{array}$ & 10 & 537 & $\begin{array}{l}\text { Std. Mean Difference (IV, Ran- } \\
\text { dom, } 95 \% \mathrm{Cl} \text { ) }\end{array}$ & $-0.56[-0.83,-0.30]$ \\
\hline
\end{tabular}




\begin{tabular}{|c|c|c|c|c|}
\hline Outcome or subgroup title & No. of studies & $\begin{array}{l}\text { No. of partici- } \\
\text { pants }\end{array}$ & Statistical method & Effect size \\
\hline $7.1 \mathrm{CBT}$ & 6 & 402 & $\begin{array}{l}\text { Std. Mean Difference (IV, Ran- } \\
\text { dom, } 95 \% \mathrm{CI} \text { ) }\end{array}$ & $-0.47[-0.81,-0.13]$ \\
\hline $7.2 \mathrm{ACT}$ & 2 & 62 & $\begin{array}{l}\text { Std. Mean Difference (IV, Ran- } \\
\text { dom, } 95 \% \mathrm{CI} \text { ) }\end{array}$ & $-0.61[-1.53,0.32]$ \\
\hline 7.3 Cognitive therapy & 1 & 40 & $\begin{array}{l}\text { Std. Mean Difference (IV, Ran- } \\
\text { dom, } 95 \% \mathrm{CI} \text { ) }\end{array}$ & $-0.97[-1.63,-0.31]$ \\
\hline 7.4 Mindfulness & 1 & 33 & $\begin{array}{l}\text { Std. Mean Difference (IV, Ran- } \\
\text { dom, } 95 \% \mathrm{CI} \text { ) }\end{array}$ & $-0.67[-1.37,0.04]$ \\
\hline $\begin{array}{l}8 \text { Subgroup analysis (fixed-effect } \\
\text { model): type of therapy - impact of } \\
\text { tinnitus on quality of life at end of } \\
\text { treatment }\end{array}$ & 10 & 537 & $\begin{array}{l}\text { Std. Mean Difference (IV, Fixed, } \\
95 \% \mathrm{CI})\end{array}$ & $-0.53[-0.71,-0.35]$ \\
\hline $8.1 \mathrm{CBT}$ & 6 & 402 & $\begin{array}{l}\text { Std. Mean Difference (IV, Fixed, } \\
95 \% \mathrm{CI})\end{array}$ & $-0.45[-0.65,-0.24]$ \\
\hline $8.2 \mathrm{ACT}$ & 2 & 62 & $\begin{array}{l}\text { Std. Mean Difference (IV, Fixed, } \\
95 \% \mathrm{CI})\end{array}$ & $-0.72[-1.24,-0.20]$ \\
\hline 8.3 Cognitive therapy & 1 & 40 & $\begin{array}{l}\text { Std. Mean Difference (IV, Fixed, } \\
95 \% \mathrm{Cl} \text { ) }\end{array}$ & $-0.97[-1.63,-0.31]$ \\
\hline 8.4 Mindfulness & 1 & 33 & $\begin{array}{l}\text { Std. Mean Difference (IV, Fixed, } \\
95 \% \mathrm{Cl} \text { ) }\end{array}$ & $-0.67[-1.37,0.04]$ \\
\hline $\begin{array}{l}9 \text { Subgroup analysis (random-ef- } \\
\text { fects model): mode of delivery - im- } \\
\text { pact of tinnitus on quality of life at } \\
\text { end of treatment }\end{array}$ & 10 & 537 & $\begin{array}{l}\text { Std. Mean Difference (IV, Ran- } \\
\text { dom, } 95 \% \mathrm{Cl} \text { ) }\end{array}$ & $-0.56[-0.83,-0.30]$ \\
\hline 9.1 Bibliotherapy & 1 & 72 & $\begin{array}{l}\text { Std. Mean Difference (IV, Ran- } \\
\text { dom, } 95 \% \mathrm{CI} \text { ) }\end{array}$ & $-0.39[-0.86,0.08]$ \\
\hline 9.2 Face-to-face & 7 & 258 & $\begin{array}{l}\text { Std. Mean Difference (IV, Ran- } \\
\text { dom, } 95 \% \mathrm{CI} \text { ) }\end{array}$ & $-0.66[-1.09,-0.22]$ \\
\hline 9.3 Internet-based CBT & 2 & 207 & $\begin{array}{l}\text { Std. Mean Difference (IV, Ran- } \\
\text { dom, } 95 \% \mathrm{CI} \text { ) }\end{array}$ & $-0.51[-0.92,-0.09]$ \\
\hline $\begin{array}{l}10 \text { Subgroup analysis (fixed-effect } \\
\text { model): mode of delivery - impact } \\
\text { of tinnitus on quality of life at end of } \\
\text { treatment }\end{array}$ & 10 & 537 & $\begin{array}{l}\text { Std. Mean Difference (IV, Fixed, } \\
95 \% \mathrm{Cl} \text { ) }\end{array}$ & $-0.53[-0.71,-0.35]$ \\
\hline 10.1 Bibliotherapy & 1 & 72 & $\begin{array}{l}\text { Std. Mean Difference (IV, Fixed, } \\
95 \% \mathrm{Cl})\end{array}$ & $-0.39[-0.86,0.08]$ \\
\hline 10.2 Face-to-face & 7 & 258 & $\begin{array}{l}\text { Std. Mean Difference (IV, Fixed, } \\
95 \% \mathrm{CI})\end{array}$ & $-0.56[-0.82,-0.30]$ \\
\hline
\end{tabular}




\begin{tabular}{|c|c|c|c|c|}
\hline Outcome or subgroup title & No. of studies & $\begin{array}{l}\text { No. of partici- } \\
\text { pants }\end{array}$ & Statistical method & Effect size \\
\hline 10.3 Web-based CBT & 2 & 207 & $\begin{array}{l}\text { Std. Mean Difference (IV, Fixed, } \\
95 \% \mathrm{CI} \text { ) }\end{array}$ & $-0.54[-0.83,-0.26]$ \\
\hline $\begin{array}{l}11 \text { Subgroup analysis (random-ef- } \\
\text { fects model): unit of delivery - im- } \\
\text { pact of tinnitus on quality of life at } \\
\text { end of treatment }\end{array}$ & 10 & 537 & $\begin{array}{l}\text { Std. Mean Difference (IV, Ran- } \\
\text { dom, } 95 \% \mathrm{Cl} \text { ) }\end{array}$ & $-0.56[-0.83,-0.30]$ \\
\hline 11.1 Individual & 4 & 323 & $\begin{array}{l}\text { Std. Mean Difference (IV, Ran- } \\
\text { dom, } 95 \% \mathrm{CI} \text { ) }\end{array}$ & $-0.57[-0.85,-0.28]$ \\
\hline 11.2 Group & 6 & 214 & $\begin{array}{l}\text { Std. Mean Difference (IV, Ran- } \\
\text { dom, 95\% CI) }\end{array}$ & $-0.59[-1.07,-0.10]$ \\
\hline $\begin{array}{l}12 \text { Subgroup analysis (fixed-effect } \\
\text { model): unit of delivery - impact of } \\
\text { tinnitus on quality of life at end of } \\
\text { treatment }\end{array}$ & 10 & 537 & $\begin{array}{l}\text { Std. Mean Difference (IV, Fixed, } \\
95 \% \mathrm{CI})\end{array}$ & $-0.53[-0.71,-0.35]$ \\
\hline 12.1 Individual & 4 & 323 & $\begin{array}{l}\text { Std. Mean Difference (IV, Fixed, } \\
95 \% \mathrm{CI})\end{array}$ & $-0.57[-0.79,-0.34]$ \\
\hline 12.2 Group & 6 & 214 & $\begin{array}{l}\text { Std. Mean Difference (IV, Fixed, } \\
95 \% \mathrm{CI})\end{array}$ & $-0.47[-0.75,-0.19]$ \\
\hline $\begin{array}{l}13 \text { Subgroup analysis (random-ef- } \\
\text { fects model): who delivers CBT - im- } \\
\text { pact of tinnitus on quality of life at } \\
\text { end of treatment }\end{array}$ & 10 & 537 & $\begin{array}{l}\text { Std. Mean Difference (IV, Ran- } \\
\text { dom, } 95 \% \mathrm{Cl} \text { ) }\end{array}$ & $-0.56[-0.83,-0.30]$ \\
\hline 13.1 Psychologist & 4 & 185 & $\begin{array}{l}\text { Std. Mean Difference (IV, Ran- } \\
\text { dom, 95\% CI) }\end{array}$ & $-0.52[-1.11,0.08]$ \\
\hline 13.2 Bibliotherapy & 1 & 72 & $\begin{array}{l}\text { Std. Mean Difference (IV, Ran- } \\
\text { dom, } 95 \% \mathrm{CI} \text { ) }\end{array}$ & $-0.39[-0.86,0.08]$ \\
\hline 13.3 Other clinician & 3 & 73 & $\begin{array}{l}\text { Std. Mean Difference (IV, Ran- } \\
\text { dom, } 95 \% \mathrm{CI} \text { ) }\end{array}$ & $-0.89[-1.51,-0.27]$ \\
\hline 13.4 Internet-based & 2 & 207 & $\begin{array}{l}\text { Std. Mean Difference (IV, Ran- } \\
\text { dom, } 95 \% \mathrm{CI} \text { ) }\end{array}$ & $-0.51[-0.92,-0.09]$ \\
\hline $\begin{array}{l}14 \text { Subgroup analysis (fixed-effect } \\
\text { model): who delivers CBT - impact } \\
\text { of tinnitus on quality of life at end of } \\
\text { treatment }\end{array}$ & 10 & 537 & $\begin{array}{l}\text { Std. Mean Difference (IV, Fixed, } \\
95 \% \mathrm{CI})\end{array}$ & $-0.53[-0.71,-0.35]$ \\
\hline 14.1 Psychologist & 4 & 185 & $\begin{array}{l}\text { Std. Mean Difference (IV, Fixed, } \\
95 \% \mathrm{CI} \text { ) }\end{array}$ & $-0.45[-0.75,-0.14]$ \\
\hline 14.2 Other clinician & 3 & 73 & $\begin{array}{l}\text { Std. Mean Difference (IV, Fixed, } \\
95 \% \mathrm{CI})\end{array}$ & $-0.87[-1.36,-0.38]$ \\
\hline 14.3 Computer & 3 & 279 & $\begin{array}{l}\text { Std. Mean Difference (IV, Fixed, } \\
95 \% \mathrm{Cl})\end{array}$ & $-0.50[-0.74,-0.26]$ \\
\hline
\end{tabular}




\begin{tabular}{lllll}
\hline Outcome or subgroup title & No. of studies & $\begin{array}{l}\text { No. of partici- } \\
\text { pants }\end{array}$ & Statistical method & Effect size \\
\hline $\begin{array}{l}15 \text { Sensitivity analysis without Mal- } \\
\text { invaud (high risk of bias) impact of } \\
\text { tinnitus on quality of life at end of } \\
\text { treatment }\end{array}$ & 9 & 454 & $\begin{array}{l}\text { Std. Mean Difference (IV, Ran- } \\
\text { dom, 95\% Cl) }\end{array}$ & $-0.64[-0.88,-0.40]$ \\
\hline $\begin{array}{l}16 \text { Sensitivity analysis with opti- } \\
\text { mistic assumption for Malinvaud - } \\
\text { impact of tinnitus on quality of life } \\
\text { at end of treatment }\end{array}$ & 10 & 526 & $\begin{array}{l}\text { Std. Mean Difference (IV, Ran- } \\
\text { dom, } 95 \% \text { CI) }\end{array}$ & $-0.65[-0.85,-0.44]$ \\
\hline
\end{tabular}

Analysis 1.1. Comparison $1 \mathrm{CBT}$ versus no intervention/waiting list control, Outcome 1 Impact of tinnitus on quality of life at end of treatment.

\begin{tabular}{|c|c|c|c|c|c|c|c|}
\hline \multirow[t]{2}{*}{ Study or subgroup } & \multicolumn{2}{|c|}{ CBT } & \multicolumn{2}{|c|}{ Wait list control } & \multirow{2}{*}{$\begin{array}{c}\text { Std. Mean Difference } \\
\text { Random, } 95 \% \mathrm{Cl}\end{array}$} & \multirow[t]{2}{*}{ Weight } & \multirow{2}{*}{$\begin{array}{c}\text { Std. Mean Difference } \\
\text { Random, } 95 \% \mathrm{Cl}\end{array}$} \\
\hline & $\mathbf{N}$ & Mean(SD) & $\mathbf{N}$ & Mean(SD) & & & \\
\hline Andersson 2002 & 24 & $29.5(22.2)$ & 48 & $35.4(23)$ & $\longrightarrow$ & $12.56 \%$ & $-0.26[-0.75,0.24]$ \\
\hline Andersson 2005 & 12 & $9.7(5.8)$ & 11 & $32.5(19)$ & - & $5.64 \%$ & $-1.6[-2.56,-0.63]$ \\
\hline Beukes 2018a & 63 & $38.7(24.3)$ & 72 & $53.7(19.4)$ & $\rightarrow$ & $16.02 \%$ & $-0.69[-1.03,-0.34]$ \\
\hline Henry 1996 & 20 & $43.7(15.5)$ & 20 & $60.9(19)$ & 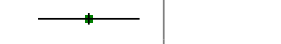 & $9.34 \%$ & $-0.97[-1.63,-0.31]$ \\
\hline Jakes 1992 & 8 & $44.6(15.4)$ & 9 & $52.8(15.4)$ & & $5.56 \%$ & $-0.5[-1.48,0.47]$ \\
\hline Kaldo 2007 & 34 & $41.7(22.9)$ & 38 & $50.6(22.5)$ & & $13.11 \%$ & $-0.39[-0.86,0.08]$ \\
\hline Kreuzer 2012 & 16 & $27.3(19.9)$ & 17 & $41.3(21.1)$ & ? & $8.64 \%$ & $-0.67[-1.37,0.04]$ \\
\hline Malinvaud 2016 & 55 & $46(20.8)$ & 28 & $45.7(22.1)$ & + & $13.39 \%$ & $0.01[-0.44,0.47]$ \\
\hline Oron (unpublished) & 10 & $50.8(20.7)$ & 8 & $52.1(13.8)$ & & $5.93 \%$ & $-0.07[-1,0.86]$ \\
\hline Westin 2011 & 22 & $27.4(19.2)$ & 22 & $48.3(21)$ & $\longrightarrow$ & $9.8 \%$ & $-1.02[-1.65,-0.39]$ \\
\hline 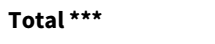 & 264 & & 273 & & & $100 \%$ & $-0.56[-0.83,-0.3]$ \\
\hline \multicolumn{8}{|c|}{ Heterogeneity: $\mathrm{Tau}^{2}=0.08 ; \mathrm{Chi}^{2}=17.62, \mathrm{df}=9(\mathrm{P}=0.04) ; \mathrm{I}^{2}=48.93 \%$} \\
\hline \multicolumn{8}{|c|}{ Test for overall effect: $Z=4.16(P<0.0001)$} \\
\hline
\end{tabular}

Analysis 1.2. Comparison 1 CBT versus no intervention/waiting list control, Outcome 2 Serious adverse effects at end of treatment.

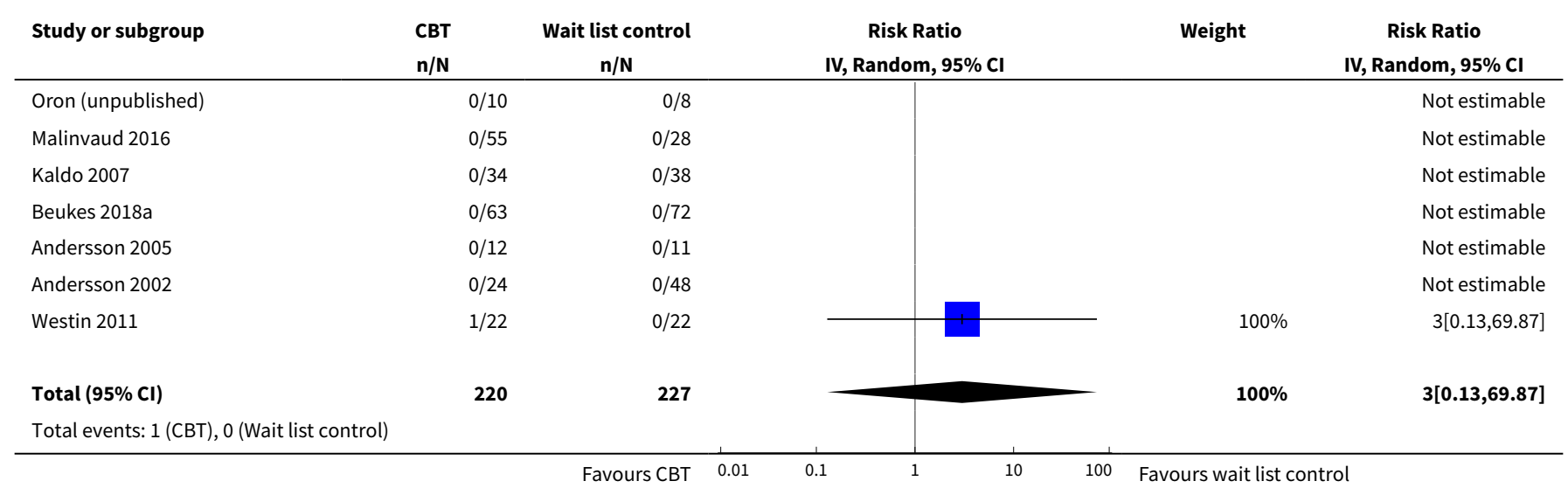




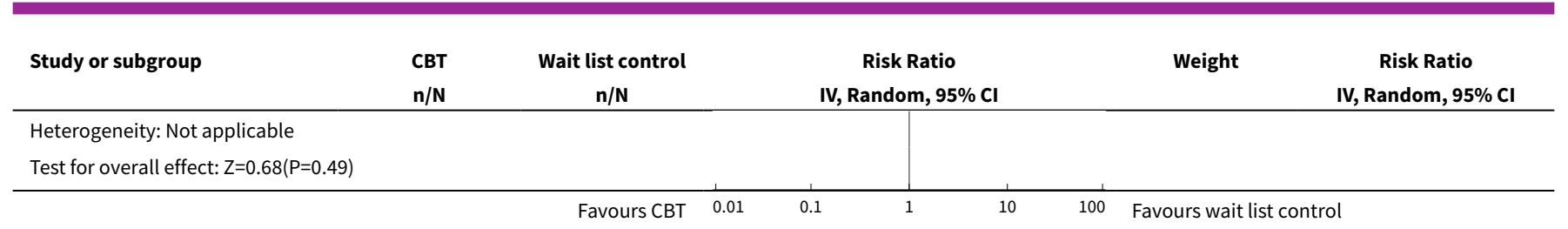

Analysis 1.3. Comparison 1 CBT versus no intervention/ waiting list control, Outcome 3 Depression at end of treatment.

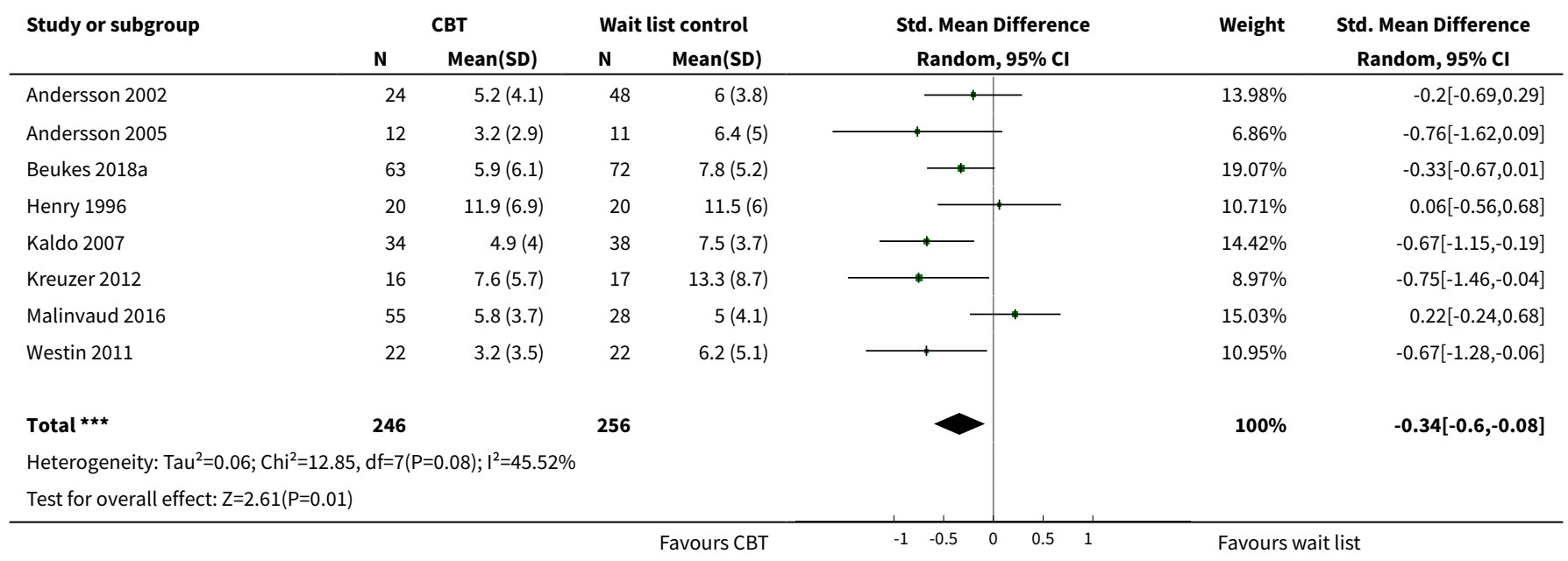

Analysis 1.4. Comparison 1 CBT versus no intervention/waiting list control, Outcome 4 Anxiety at end of treatment.

\begin{tabular}{|c|c|c|c|c|c|c|c|}
\hline \multirow[t]{2}{*}{ Study or subgroup } & \multicolumn{2}{|c|}{ CBT } & \multicolumn{2}{|c|}{ Wait list control } & \multirow{2}{*}{$\begin{array}{c}\text { Std. Mean Difference } \\
\text { Random, } 95 \% \mathrm{Cl}\end{array}$} & \multirow[t]{2}{*}{ Weight } & \multirow{2}{*}{$\begin{array}{c}\text { Std. Mean Difference } \\
\text { Random, } 95 \% \mathrm{Cl}\end{array}$} \\
\hline & $\mathbf{N}$ & Mean(SD) & $\mathbf{N}$ & Mean(SD) & & & \\
\hline Andersson 2002 & 24 & $5.9(3.6)$ & 48 & $6.8(4.8)$ & $\rightarrow$ & $17.71 \%$ & $-0.2[-0.69,0.29]$ \\
\hline Andersson 2005 & 12 & $2.7(1.4)$ & 11 & $6.7(3.5)$ & $*$ & $9.53 \%$ & $-1.47[-2.41,-0.53]$ \\
\hline Beukes 2018a & 63 & $5.5(5.2)$ & 72 & $6.9(4.9)$ & $\rightarrow$ & $21.26 \%$ & $-0.27[-0.61,0.07]$ \\
\hline Kaldo 2007 & 34 & $6.2(4.4)$ & 38 & $9.6(4.7)$ & 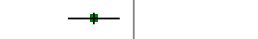 & $17.99 \%$ & $-0.74[-1.22,-0.26]$ \\
\hline Malinvaud 2016 & 55 & $9.5(4.1)$ & 28 & $8.8(4.1)$ & $\rightarrow$ & $18.53 \%$ & $0.17[-0.29,0.62]$ \\
\hline Westin 2011 & 22 & $3.6(3.1)$ & 22 & $7.2(5.6)$ & $\longrightarrow$ & $14.98 \%$ & $-0.78[-1.4,-0.17]$ \\
\hline 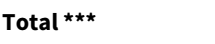 & 210 & & 219 & & & $100 \%$ & $-0.45[-0.82,-0.09]$ \\
\hline \multicolumn{8}{|c|}{ Heterogeneity: $\operatorname{Tau}^{2}=0.13 ; \mathrm{Chi}^{2}=15.36, \mathrm{df}=5(\mathrm{P}=0.01) ; \mathrm{I}^{2}=67.46 \%$} \\
\hline Test for overall effect & & & & & & & \\
\hline
\end{tabular}


Analysis 1.5. Comparison $1 \mathrm{CBT}$ versus no intervention/waiting list control, Outcome 5 Health-related quality of life at end of treatment.

\begin{tabular}{|c|c|c|c|c|c|c|c|}
\hline \multirow[t]{2}{*}{ Study or subgroup } & \multicolumn{2}{|c|}{ CBT } & \multicolumn{2}{|c|}{ Wait list control } & \multirow{2}{*}{$\begin{array}{c}\text { Std. Mean Difference } \\
\text { Random, } 95 \% \mathrm{Cl}\end{array}$} & \multirow[t]{2}{*}{ Weight } & \multirow{2}{*}{$\begin{array}{c}\text { Std. Mean Difference } \\
\text { Random, } 95 \% \mathrm{Cl}\end{array}$} \\
\hline & $\mathbf{N}$ & Mean(SD) & $\mathbf{N}$ & Mean(SD) & & & \\
\hline Beukes 2018a & 63 & $-18.3(6.7)$ & 72 & $-16.2(6.3)$ & & $75.73 \%$ & $-0.33[-0.67,0.01]$ \\
\hline Westin 2011 & 22 & $-2.8(1.5)$ & 22 & $-1.9(1.8)$ & $\longrightarrow$ & $24.27 \%$ & $-0.51[-1.11,0.09]$ \\
\hline Total $\star \star \star ~$ & 85 & & 94 & & & $100 \%$ & $-0.38[-0.67,-0.08]$ \\
\hline \multicolumn{8}{|c|}{ Heterogeneity: $\mathrm{Tau}^{2}=0 ; \mathrm{Chi}^{2}=0.26, \mathrm{df}=1(\mathrm{P}=0.61) ;\left.\right|^{2}=0 \%$} \\
\hline \multicolumn{3}{|c|}{ Test for overall effect: $Z=2.48(P=0.01)$} & & & & & \\
\hline
\end{tabular}

Analysis 1.6. Comparison $1 \mathrm{CBT}$ versus no intervention/waiting list control, Outcome 6 Negatively biased interpretations of tinnitus at end of treatment.

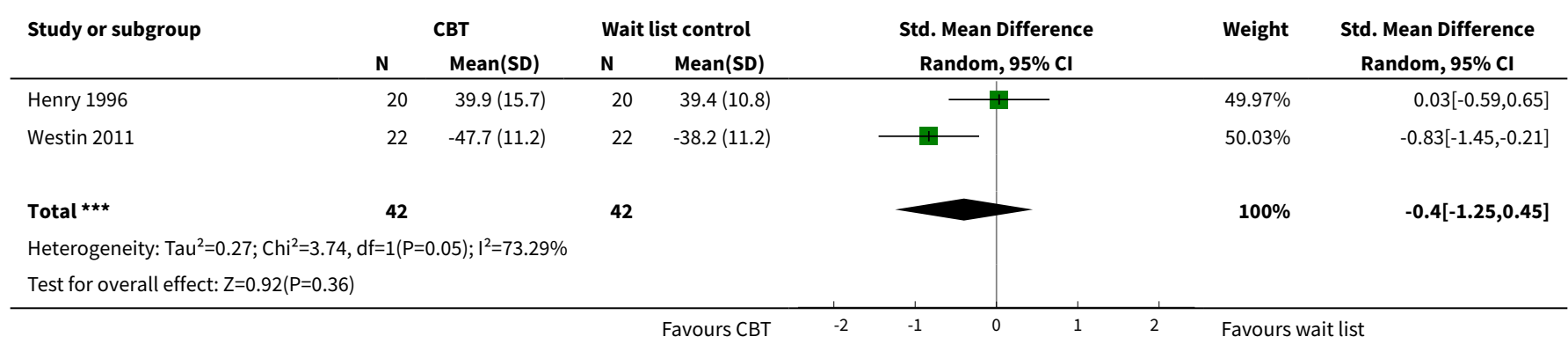

Analysis 1.7. Comparison 1 CBT versus no intervention/waiting list control, Outcome 7 Subgroup analysis (random-effects model): type of therapy - impact of tinnitus on quality of life at end of treatment.

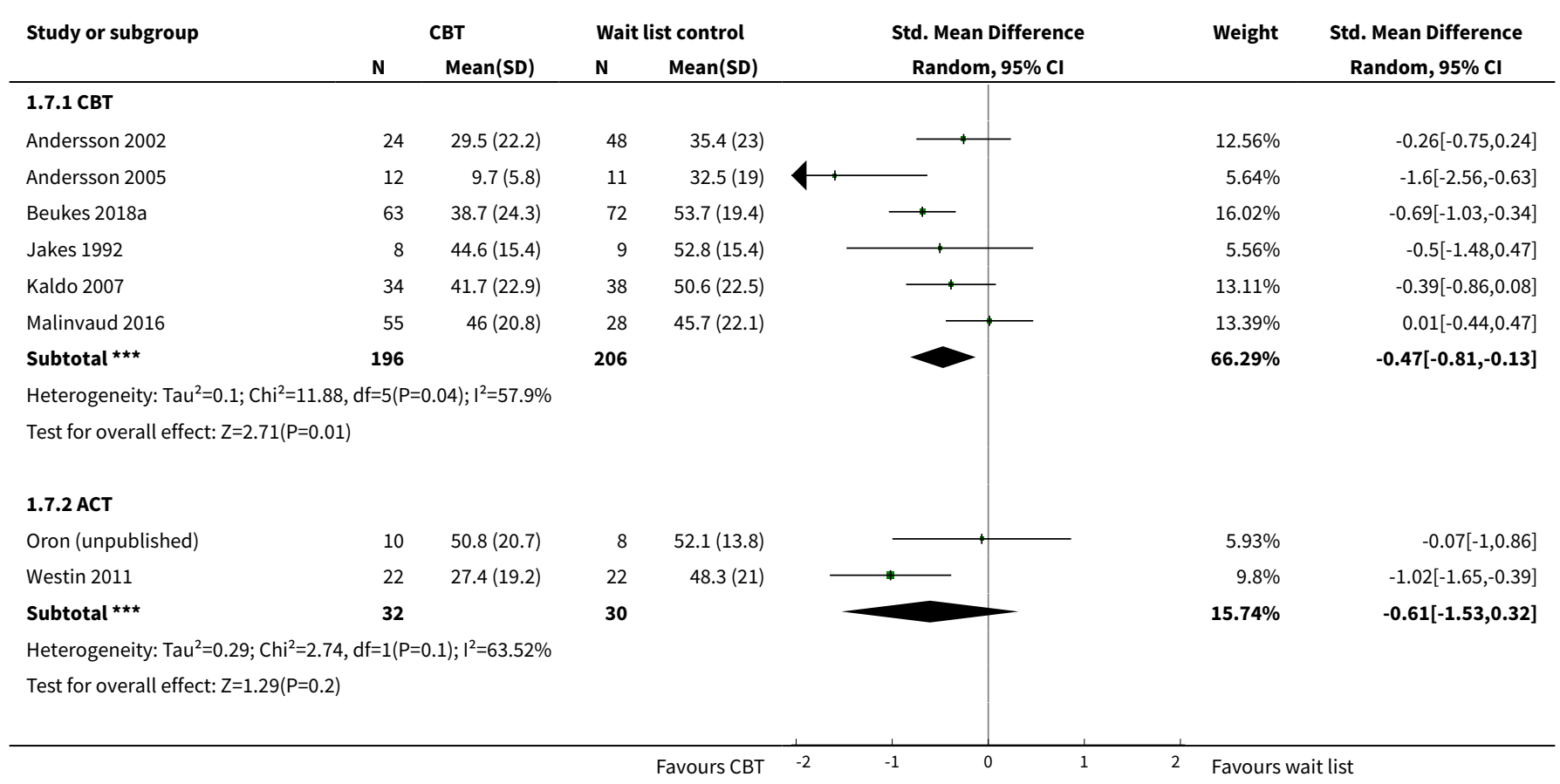




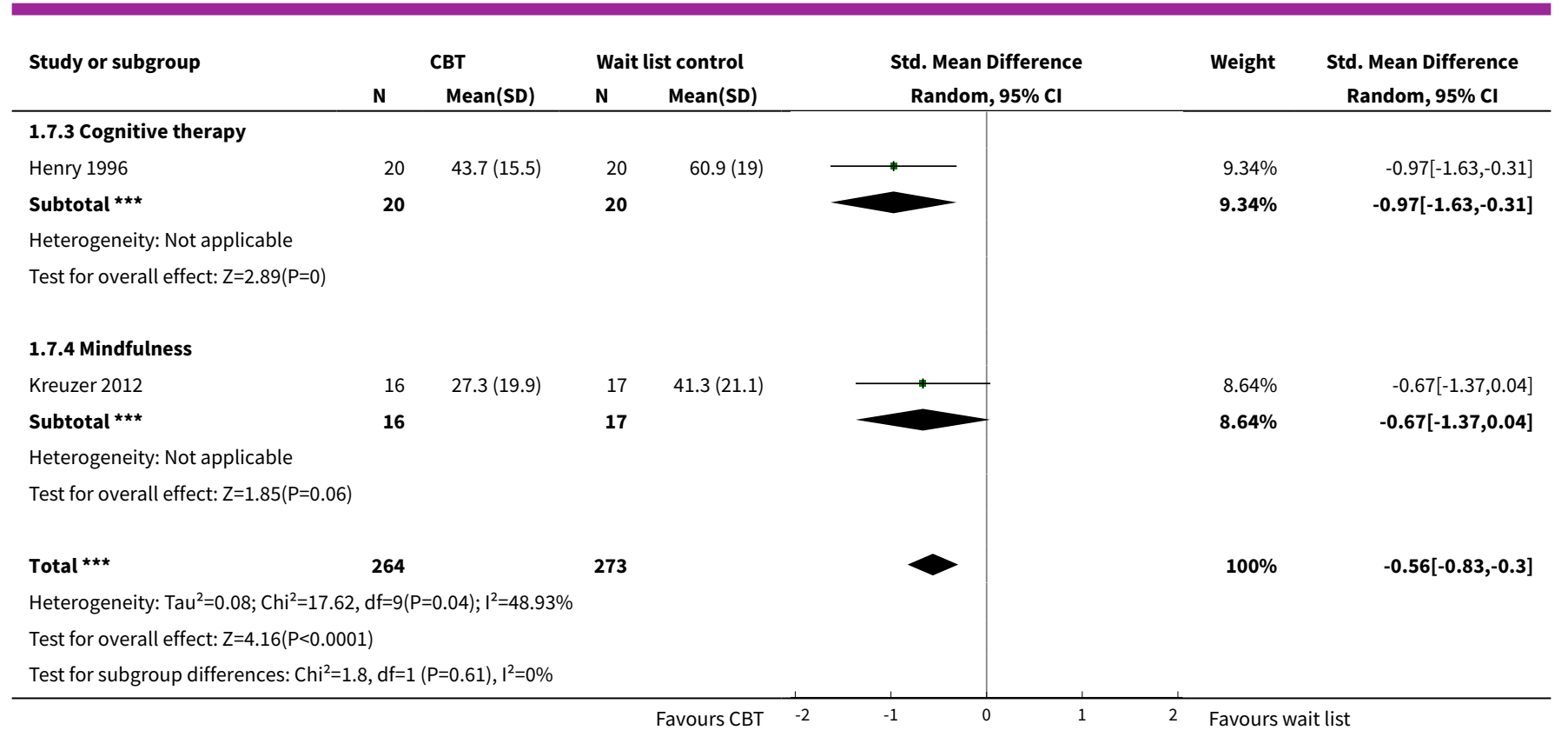

Analysis 1.8. Comparison 1 CBT versus no intervention/waiting list control, Outcome 8 Subgroup analysis (fixed-effect model): type of therapy - impact of tinnitus on quality of life at end of treatment.

\begin{tabular}{|c|c|c|c|c|c|c|c|}
\hline \multirow[t]{2}{*}{ Study or subgroup } & \multicolumn{2}{|c|}{ CBT } & \multicolumn{2}{|c|}{ Wait list control } & \multirow{2}{*}{$\begin{array}{c}\text { Std. Mean Difference } \\
\text { Fixed, } 95 \% \mathrm{Cl}\end{array}$} & \multirow[t]{2}{*}{ Weight } & \multirow{2}{*}{$\begin{array}{c}\text { Std. Mean Difference } \\
\text { Fixed, } 95 \% \mathrm{Cl}\end{array}$} \\
\hline & $\mathbf{N}$ & Mean(SD) & $\mathbf{N}$ & Mean(SD) & & & \\
\hline \multicolumn{8}{|l|}{ 1.8.1 CBT } \\
\hline Andersson 2005 & 12 & $9.7(5.8)$ & 11 & $32.5(19)$ & & $3.38 \%$ & $-1.6[-2.56,-0.63]$ \\
\hline Beukes 2018a & 63 & $38.7(24.3)$ & 72 & $53.7(19.4)$ & \pm & $25.85 \%$ & $-0.69[-1.03,-0.34]$ \\
\hline Jakes 1992 & 8 & $44.6(15.4)$ & 9 & $52.8(15.4)$ & \begin{tabular}{l|l}
+ & \\
\end{tabular} & $3.32 \%$ & $-0.5[-1.48,0.47]$ \\
\hline Kaldo 2007 & 34 & 41.7 (22.9) & 38 & $50.6(22.5)$ & $\rightarrow$ & $14.35 \%$ & $-0.39[-0.86,0.08]$ \\
\hline Malinvaud 2016 & 55 & $46(20.8)$ & 28 & $45.7(22.1)$ & $\longrightarrow$ & $15.13 \%$ & $0.01[-0.44,0.47]$ \\
\hline Subtotal $* \star \star$ & 196 & & 206 & & $\diamond$ & $74.98 \%$ & $-0.45[-0.65,-0.24]$ \\
\hline \multicolumn{8}{|c|}{ Heterogeneity: $\mathrm{Tau}^{2}=0 ; \mathrm{Chi}^{2}=11.88, \mathrm{df}=5(\mathrm{P}=0.04) ; \mathrm{I}^{2}=57.9 \%$} \\
\hline \multicolumn{8}{|c|}{ Test for overall effect: $Z=4.29(P<0.0001)$} \\
\hline \multicolumn{8}{|l|}{ 1.8.2 ACT } \\
\hline Westin 2011 & 22 & $27.4(19.2)$ & 22 & $48.3(21)$ & $\longrightarrow$ & $7.86 \%$ & $-1.02[-1.65,-0.39]$ \\
\hline 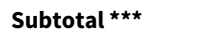 & 32 & & 30 & & & $11.48 \%$ & $-0.72[-1.24,-0.2]$ \\
\hline \multicolumn{8}{|c|}{ Heterogeneity: $\mathrm{Tau}^{2}=0 ; \mathrm{Chi}^{2}=2.74, \mathrm{df}=1(\mathrm{P}=0.1) ; \mathrm{I}^{2}=63.52 \%$} \\
\hline \multicolumn{8}{|c|}{ Test for overall effect: $Z=2.69(P=0.01)$} \\
\hline \multicolumn{8}{|c|}{ 1.8.3 Cognitive therapy } \\
\hline Henry 1996 & 20 & $43.7(15.5)$ & 20 & $60.9(19)$ & $\longrightarrow$ & $7.21 \%$ & $-0.97[-1.63,-0.31]$ \\
\hline Subtotal $\star \star \star$ & 20 & & 20 & & & $7.21 \%$ & $-0.97[-1.63,-0.31]$ \\
\hline \multicolumn{8}{|c|}{ Heterogeneity: Not applicable } \\
\hline \multicolumn{8}{|c|}{ Test for overall effect: $Z=2.89(P=0)$} \\
\hline \multicolumn{8}{|l|}{ 1.8.4 Mindfulness } \\
\hline Kreuzer 2012 & 16 & $27.3(19.9)$ & 17 & $41.3(21.1)$ & - & $6.33 \%$ & $-0.67[-1.37,0.04]$ \\
\hline
\end{tabular}




\begin{tabular}{|c|c|c|c|c|c|c|}
\hline \multirow[t]{2}{*}{ Study or subgroup } & \multicolumn{2}{|c|}{ СВT } & Wait list control & \multirow{2}{*}{$\begin{array}{l}\text { Std. Mean Difference } \\
\text { Fixed, } 95 \% \mathrm{Cl}\end{array}$} & \multirow[t]{2}{*}{ Weight } & \multirow{2}{*}{$\begin{array}{l}\text { Std. Mean Difference } \\
\text { Fixed, } 95 \% \mathrm{Cl}\end{array}$} \\
\hline & $\mathbf{N}$ & Mean(SD) & Mean(SD) & & & \\
\hline Subtotal $\star \star \star ~$ & 16 & & 17 & & $6.33 \%$ & $-0.67[-1.37,0.04]$ \\
\hline \multicolumn{7}{|c|}{ Heterogeneity: Not applicable } \\
\hline \multicolumn{7}{|c|}{ Test for overall effect: $Z=1.85(P=0.06)$} \\
\hline Total $\star \star \star$ & 264 & & 273 & $\diamond$ & $100 \%$ & $-0.53[-0.71,-0.35]$ \\
\hline \multicolumn{7}{|c|}{ Heterogeneity: $\operatorname{Tau}^{2}=0 ; \mathrm{Chi}^{2}=17.62, \mathrm{df}=9(\mathrm{P}=0.04) ;\left.\right|^{2}=48.93 \%$} \\
\hline \multicolumn{7}{|c|}{ Test for overall effect: $Z=5.87(P<0.0001)$} \\
\hline \multicolumn{7}{|c|}{ Test for subgroup differences: $\mathrm{Chi}^{2}=3, \mathrm{df}=1(\mathrm{P}=0.39), \mathrm{I}^{2}=0.12 \%$} \\
\hline & & & Favours CBT & -1 & Favours & list \\
\hline
\end{tabular}

\section{Analysis 1.9. Comparison 1 CBT versus no intervention/waiting list control, Outcome 9 Subgroup analysis (random-effects model): mode of delivery - impact of tinnitus on quality of life at end of treatment.}

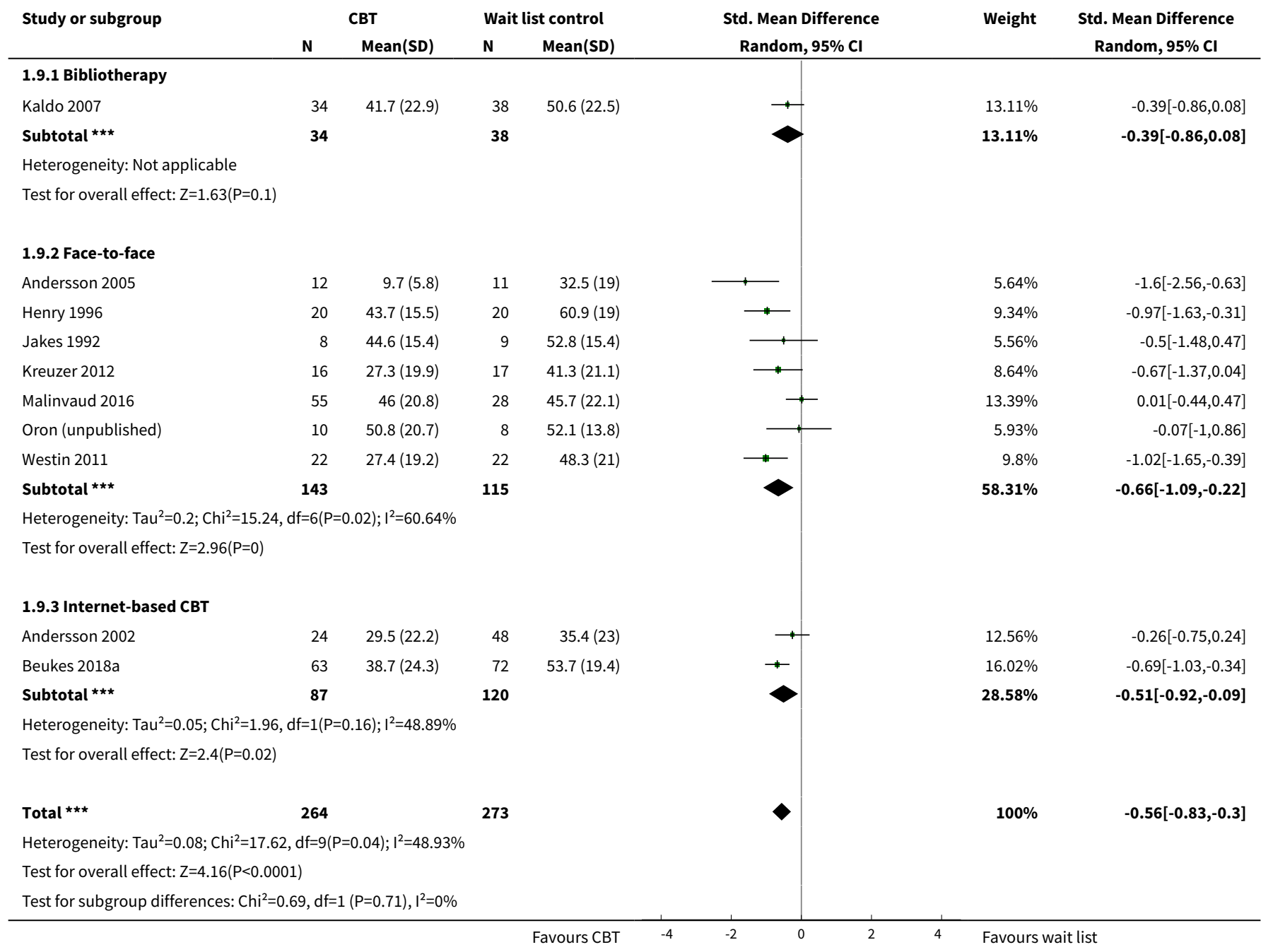


Analysis 1.10. Comparison 1 CBT versus no intervention/waiting list control, Outcome 10 Subgroup analysis (fixed-effect model): mode of delivery - impact of tinnitus on quality of life at end of treatment.

\begin{tabular}{|c|c|c|c|c|c|c|c|}
\hline \multirow{3}{*}{$\begin{array}{l}\text { Study or subgroup } \\
\text { 1.10.1 Bibliotherapy }\end{array}$} & \multicolumn{2}{|c|}{ СBT } & \multicolumn{2}{|c|}{ Wait list control } & \multirow{2}{*}{$\begin{array}{l}\text { Std. Mean Difference } \\
\text { Fixed, } 95 \% \mathrm{Cl}\end{array}$} & \multirow[t]{2}{*}{ Weight } & \multirow{2}{*}{$\begin{array}{l}\text { Std. Mean Difference } \\
\text { Fixed, } 95 \% \mathrm{Cl}\end{array}$} \\
\hline & \multirow[t]{2}{*}{$\mathbf{N}$} & \multirow[t]{2}{*}{ Mean(SD) } & \multirow[t]{2}{*}{$\mathbf{N}$} & \multirow[t]{2}{*}{$\operatorname{Mean}(S D)$} & & & \\
\hline & & & & & & & \\
\hline Kaldo 2007 & 34 & $41.7(22.9)$ & 38 & $50.6(22.5)$ & $\rightarrow$ & $14.35 \%$ & $-0.39[-0.86,0.08]$ \\
\hline 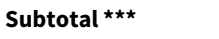 & 34 & & 38 & & & $14.35 \%$ & $-0.39[-0.86,0.08]$ \\
\hline \multicolumn{8}{|c|}{ Heterogeneity: Not applicable } \\
\hline \multicolumn{8}{|c|}{ Test for overall effect: $Z=1.63(P=0.1)$} \\
\hline \multicolumn{8}{|l|}{ 1.10.2 Face-to-face } \\
\hline Andersson 2005 & 12 & $9.7(5.8)$ & 11 & $32.5(19)$ & 1 & $3.38 \%$ & $-1.6[-2.56,-0.63]$ \\
\hline Henry 1996 & 20 & $43.7(15.5)$ & 20 & $60.9(19)$ & 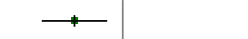 & $7.21 \%$ & $-0.97[-1.63,-0.31]$ \\
\hline Jakes 1992 & 8 & $44.6(15.4)$ & 9 & $52.8(15.4)$ & - & $3.32 \%$ & $-0.5[-1.48,0.47]$ \\
\hline Kreuzer 2012 & 16 & $27.3(19.9)$ & 17 & $41.3(21.1)$ & & $6.33 \%$ & $-0.67[-1.37,0.04]$ \\
\hline Malinvaud 2016 & 55 & $46(20.8)$ & 28 & $45.7(22.1)$ & + & $15.13 \%$ & $0.01[-0.44,0.47]$ \\
\hline Westin 2011 & 22 & $27.4(19.2)$ & 22 & $48.3(21)$ & $\longrightarrow$ & $7.86 \%$ & $-1.02[-1.65,-0.39]$ \\
\hline Subtotal $\star \star \star ~$ & 143 & & 115 & & $>$ & $46.85 \%$ & $-0.56[-0.82,-0.3]$ \\
\hline \multicolumn{8}{|c|}{ Heterogeneity: $\operatorname{Tau}^{2}=0 ; \mathrm{Chi}^{2}=15.24, \mathrm{df}=6(\mathrm{P}=0.02) ; \mathrm{I}^{2}=60.64 \%$} \\
\hline \multicolumn{8}{|c|}{ Test for overall effect: $Z=4.26(P<0.0001)$} \\
\hline \multicolumn{8}{|c|}{ 1.10.3 Web-based CBT } \\
\hline Andersson 2002 & 24 & $29.5(22.2)$ & 48 & $35.4(23)$ & $\rightarrow$ & $12.95 \%$ & $-0.26[-0.75,0.24]$ \\
\hline Beukes 2018a & 63 & $38.7(24.3)$ & 72 & $53.7(19.4)$ & $\rightarrow$ & $25.85 \%$ & $-0.69[-1.03,-0.34]$ \\
\hline Subtotal $\star \star \star ~$ & 87 & & 120 & & $>$ & $38.8 \%$ & $-0.54[-0.83,-0.26]$ \\
\hline \multicolumn{8}{|c|}{ Heterogeneity: $\mathrm{Tau}^{2}=0 ; \mathrm{Chi}^{2}=1.96, \mathrm{df}=1(\mathrm{P}=0.16) ; \mathrm{I}^{2}=48.89 \%$} \\
\hline \multicolumn{8}{|c|}{ Test for overall effect: $Z=3.75(P=0)$} \\
\hline Total $\star \star \star$ & 264 & & 273 & & $\diamond$ & $100 \%$ & $-0.53[-0.71,-0.35]$ \\
\hline Test for subgroup diff & $42, \mathrm{df}=$ & $P=0.81), I^{2}=0 \%$ & & & & & \\
\hline
\end{tabular}

Analysis 1.11. Comparison 1 CBT versus no intervention/waiting list control, Outcome 11 Subgroup analysis (random-effects model): unit of delivery - impact of tinnitus on quality of life at end of treatment.

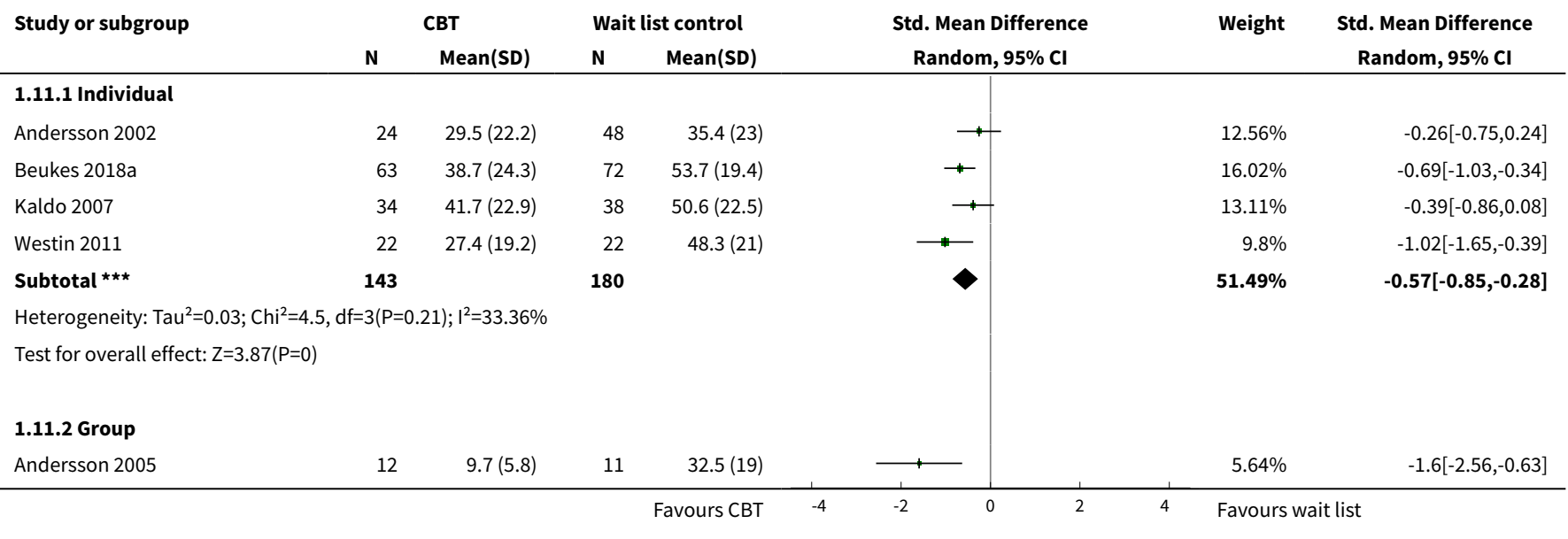




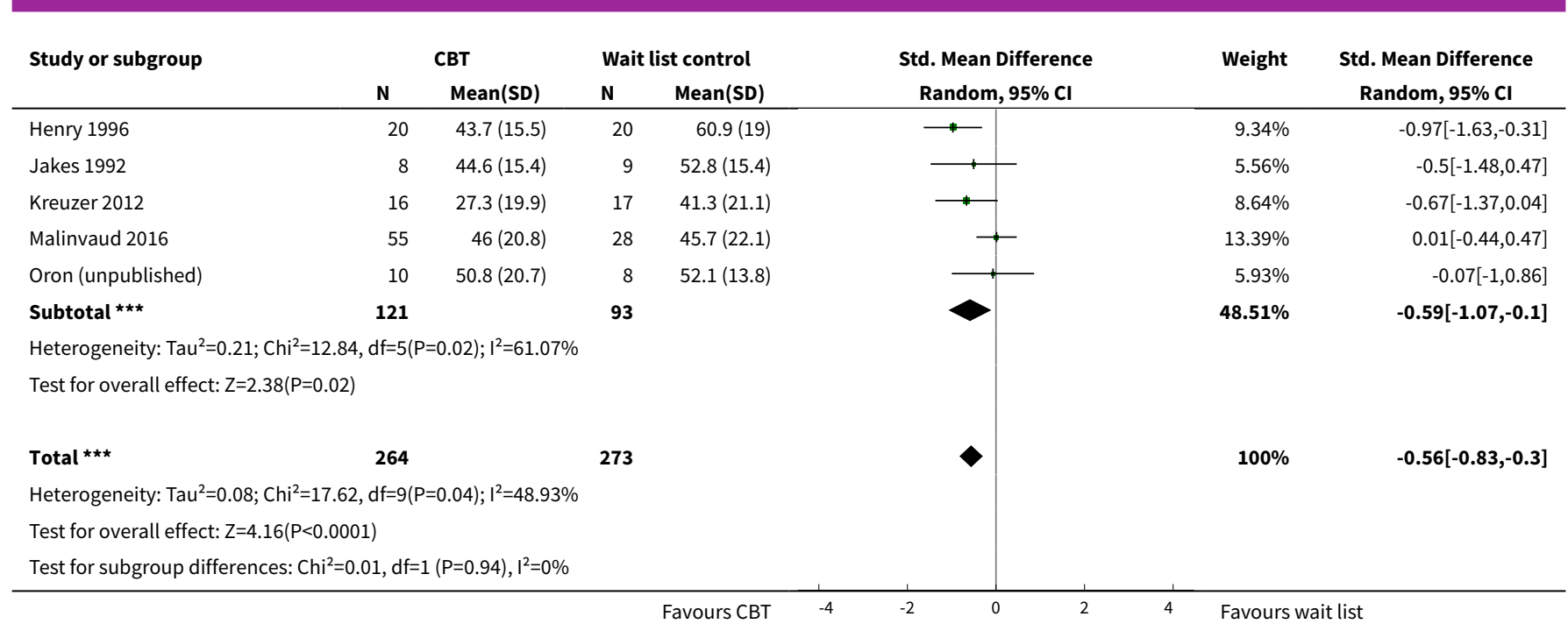

Analysis 1.12. Comparison 1 CBT versus no intervention/waiting list control, Outcome 12 Subgroup analysis (fixed-effect model): unit of delivery - impact of tinnitus on quality of life at end of treatment.

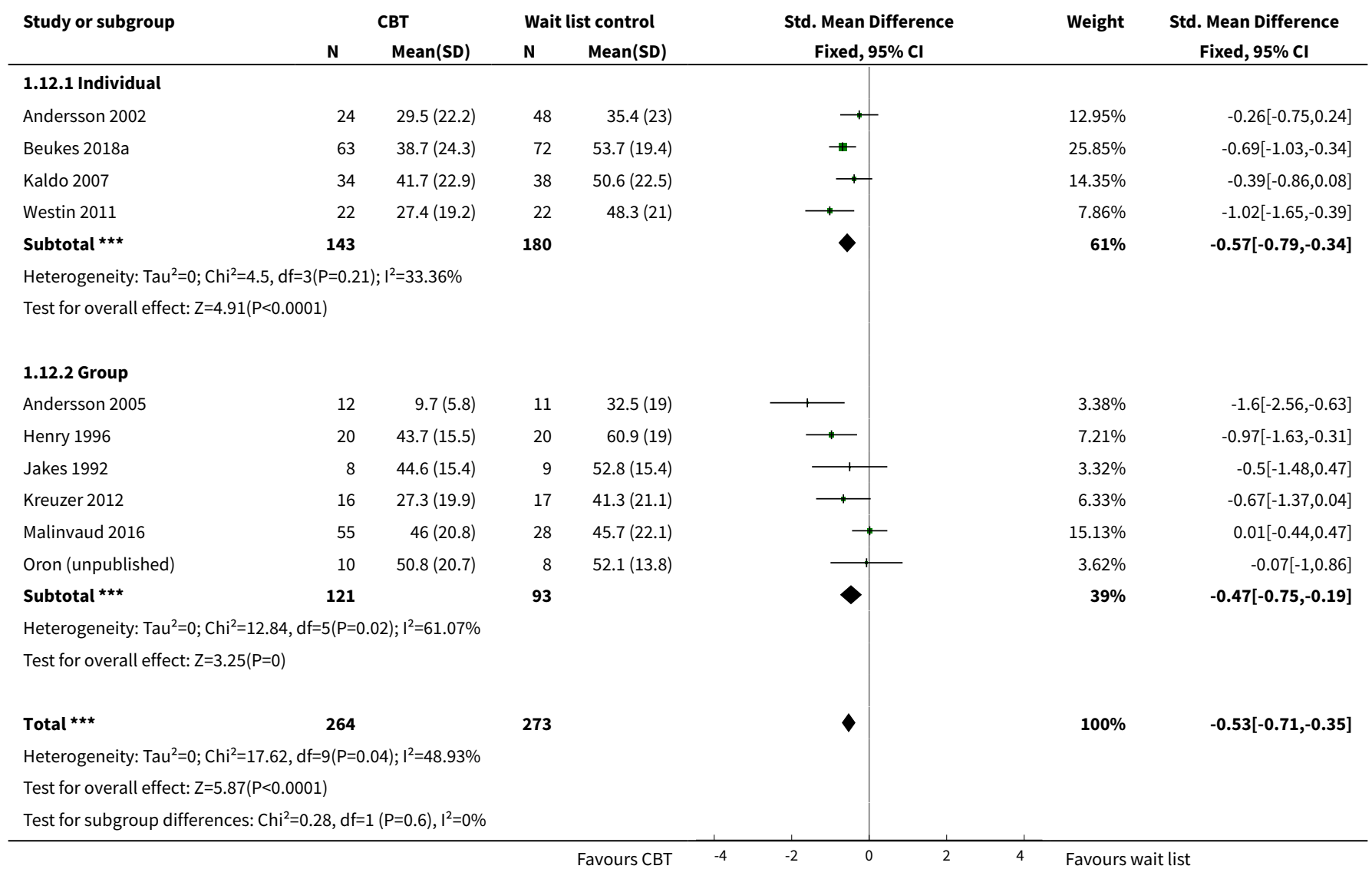


Analysis 1.13. Comparison 1 CBT versus no intervention/waiting list control, Outcome 13 Subgroup analysis (random-effects model): who delivers CBT - impact of tinnitus on quality of life at end of treatment.

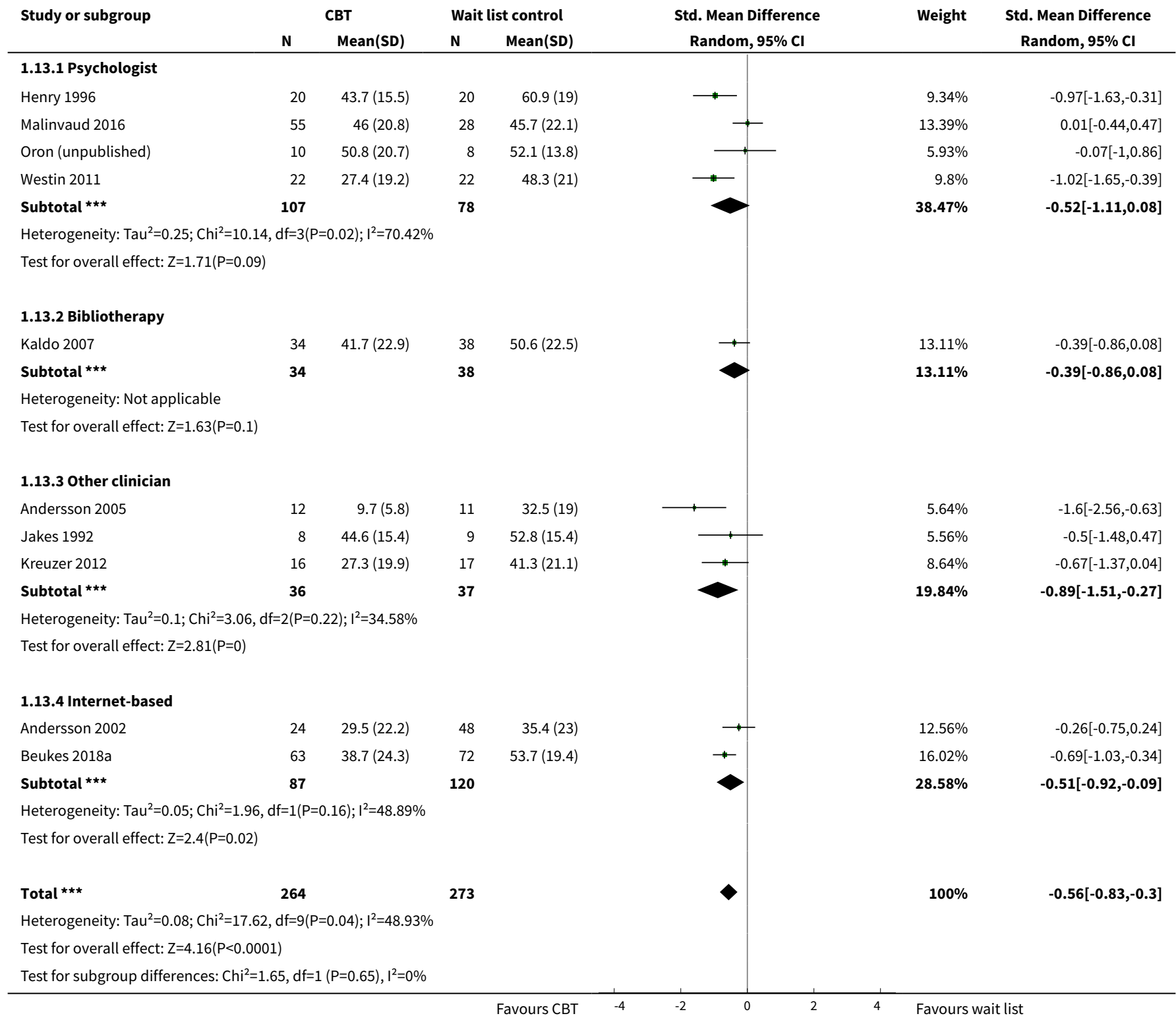

Analysis 1.14. Comparison 1 CBT versus no intervention/waiting list control, Outcome 14 Subgroup analysis (fixed-effect model): who delivers CBT - impact of tinnitus on quality of life at end of treatment.

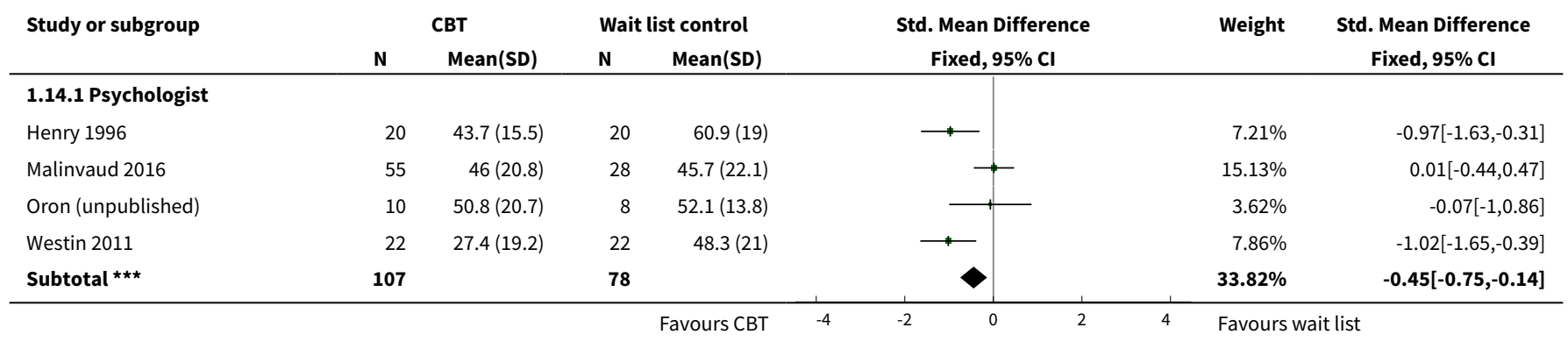




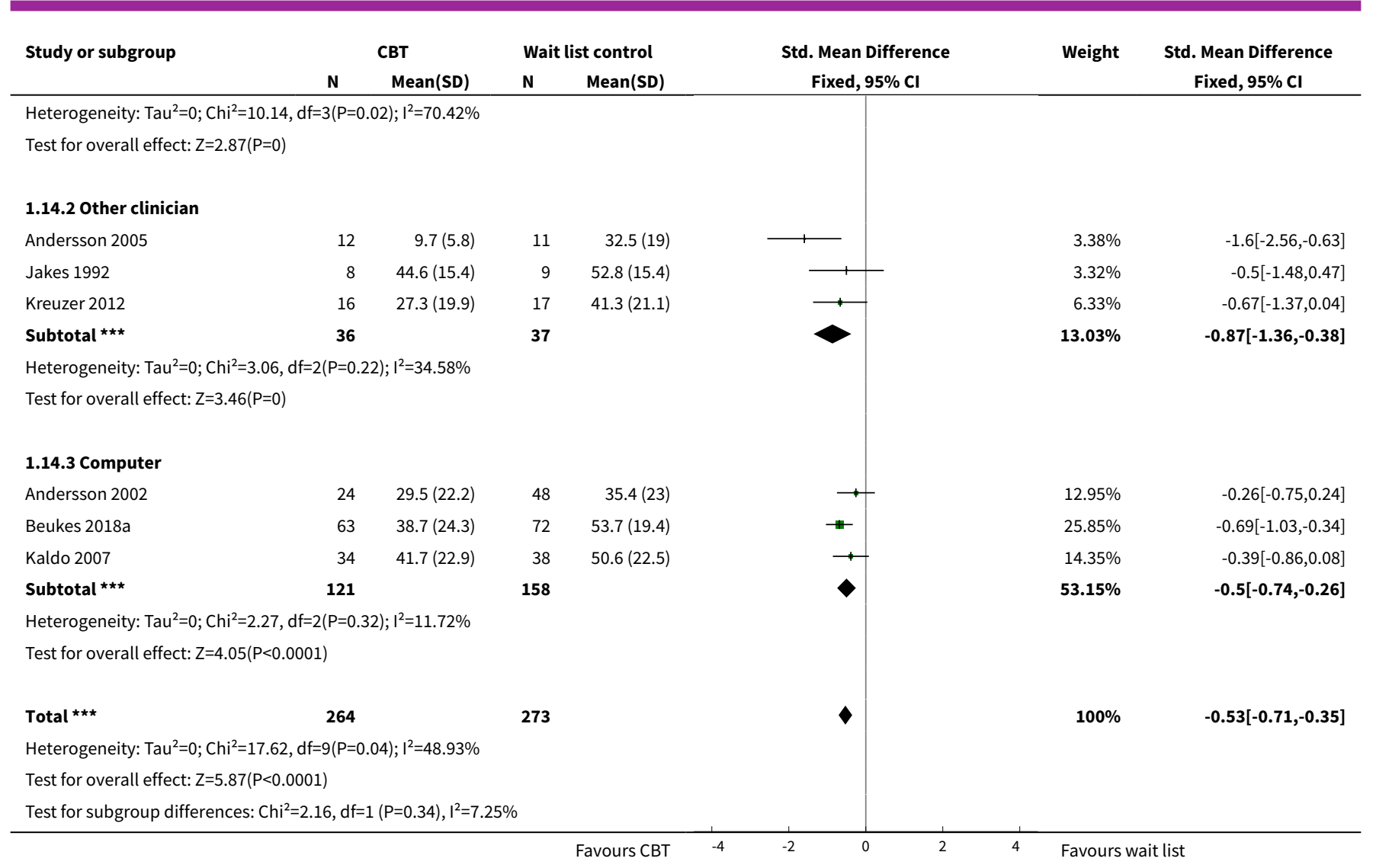

Analysis 1.15. Comparison 1 CBT versus no intervention/waiting list control, Outcome 15 Sensitivity analysis without Malinvaud (high risk of bias) impact of tinnitus on quality of life at end of treatment.

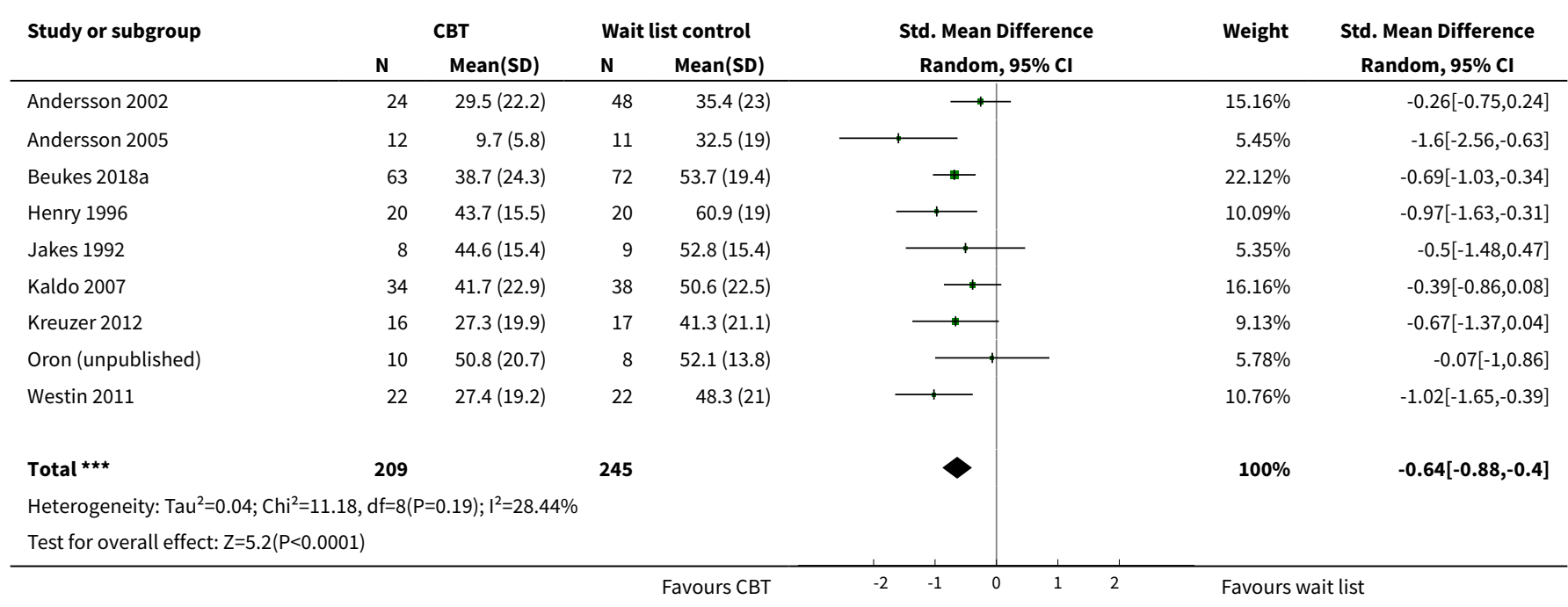


Analysis 1.16. Comparison 1 CBT versus no intervention/waiting list control, Outcome 16 Sensitivity analysis with optimistic assumption for Malinvaud - impact of tinnitus on quality of life at end of treatment.

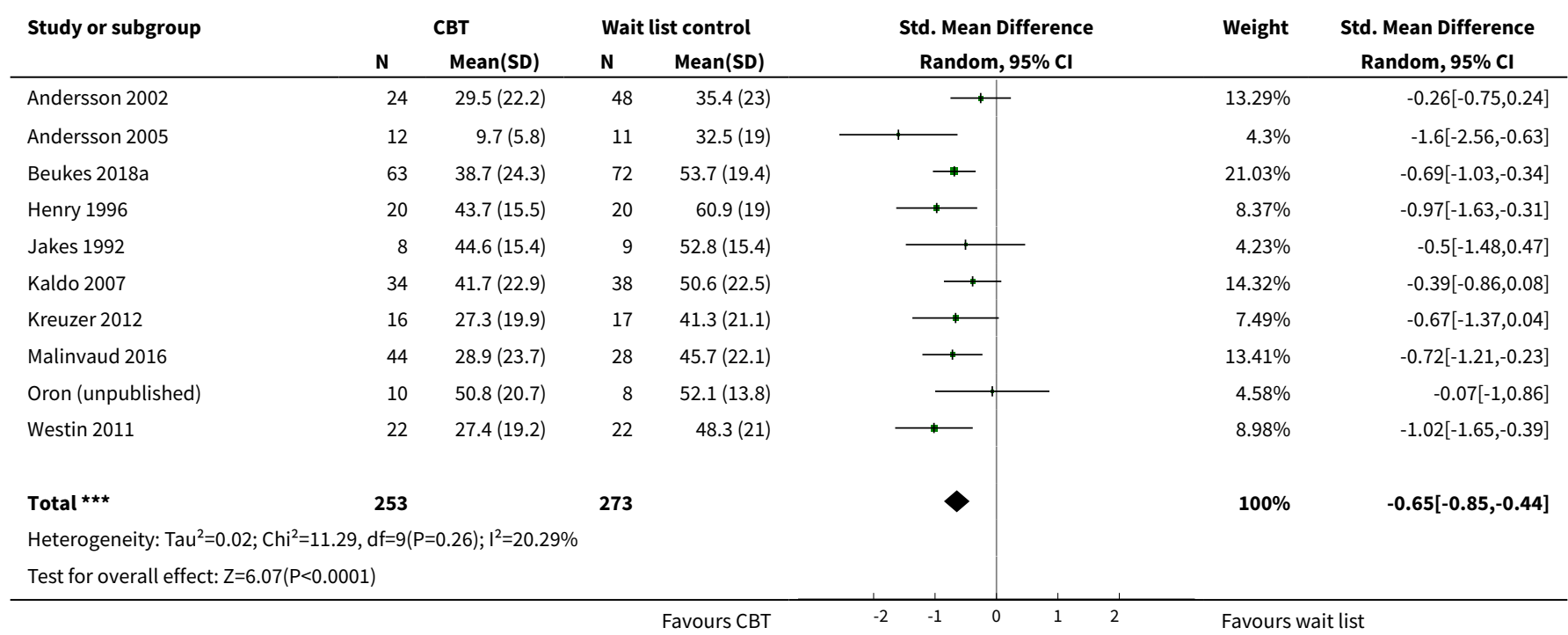

Comparison 2. CBT versus audiological care (tinnitus education and rehabilitation for hearing loss)

\begin{tabular}{|c|c|c|c|c|}
\hline Outcome or subgroup title & No. of studies & $\begin{array}{l}\text { No. of partici- } \\
\text { pants }\end{array}$ & Statistical method & Effect size \\
\hline $\begin{array}{l}1 \text { Impact of tinnitus on quality of life } \\
\text { at end of treatment }\end{array}$ & 3 & 444 & $\begin{array}{l}\text { Mean Difference (IV, Random, } \\
95 \% \mathrm{CI})\end{array}$ & $-5.68[-9.74,-1.61]$ \\
\hline $\begin{array}{l}2 \text { Serious adverse effects at end of } \\
\text { treatment }\end{array}$ & 2 & 410 & Risk Ratio (M-H, Fixed, 95\% Cl) & $0.0[0.0,0.0]$ \\
\hline 3 Depression at end of treatment & 2 & 410 & $\begin{array}{l}\text { Std. Mean Difference (IV, Ran- } \\
\text { dom, } 95 \% \mathrm{CI} \text { ) }\end{array}$ & $-0.18[-0.38,0.01]$ \\
\hline 4 Anxiety at end of treatment & 2 & 410 & $\begin{array}{l}\text { Std. Mean Difference (IV, Ran- } \\
\text { dom, } 95 \% \mathrm{CI} \text { ) }\end{array}$ & $-0.06[-0.26,0.13]$ \\
\hline 5 Health-related quality of life & 2 & 410 & $\begin{array}{l}\text { Std. Mean Difference (IV, Ran- } \\
\text { dom, } 95 \% \mathrm{CI} \text { ) }\end{array}$ & $-0.07[-0.26,0.13]$ \\
\hline $\begin{array}{l}6 \text { Negatively biased interpretations } \\
\text { of tinnitus }\end{array}$ & 1 & 336 & $\begin{array}{l}\text { Mean Difference (IV, Random, } \\
95 \% \mathrm{CI} \text { ) }\end{array}$ & $-4.69[-7.04,-2.34]$ \\
\hline $\begin{array}{l}7 \text { Sensitivity analysis (fixed-effect } \\
\text { model): impact of tinnitus on quali- } \\
\text { ty of life }\end{array}$ & 3 & 430 & $\begin{array}{l}\text { Mean Difference (IV, Fixed, 95\% } \\
\mathrm{CI} \text { ) }\end{array}$ & $-5.65[-9.79,-1.50]$ \\
\hline
\end{tabular}


Analysis 2.1. Comparison 2 CBT versus audiological care (tinnitus education and rehabilitation for hearing loss), Outcome 1 Impact of tinnitus on quality of life at end of treatment.

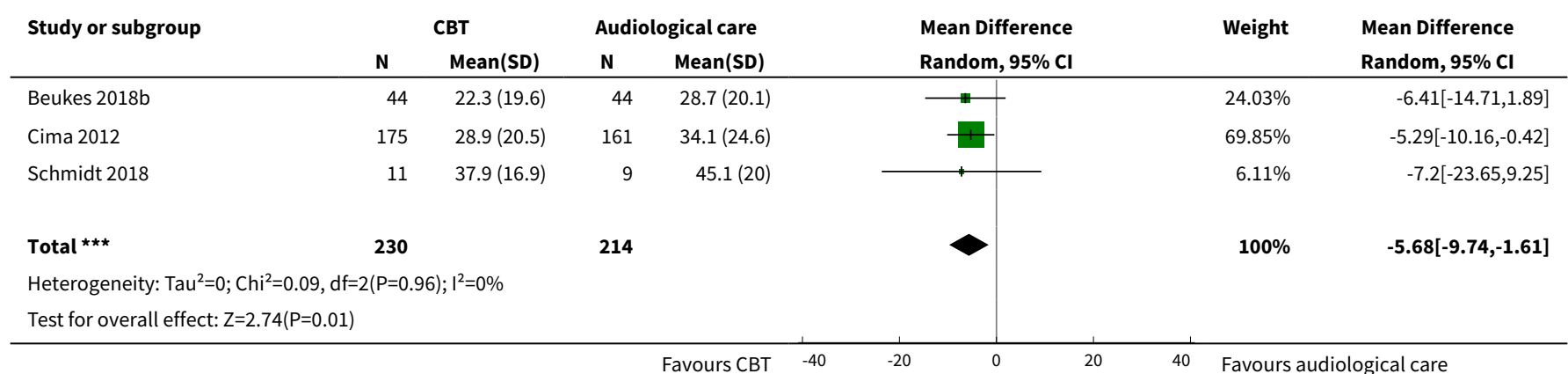

Analysis 2.2. Comparison 2 CBT versus audiological care (tinnitus education and rehabilitation for hearing loss), Outcome 2 Serious adverse effects at end of treatment.

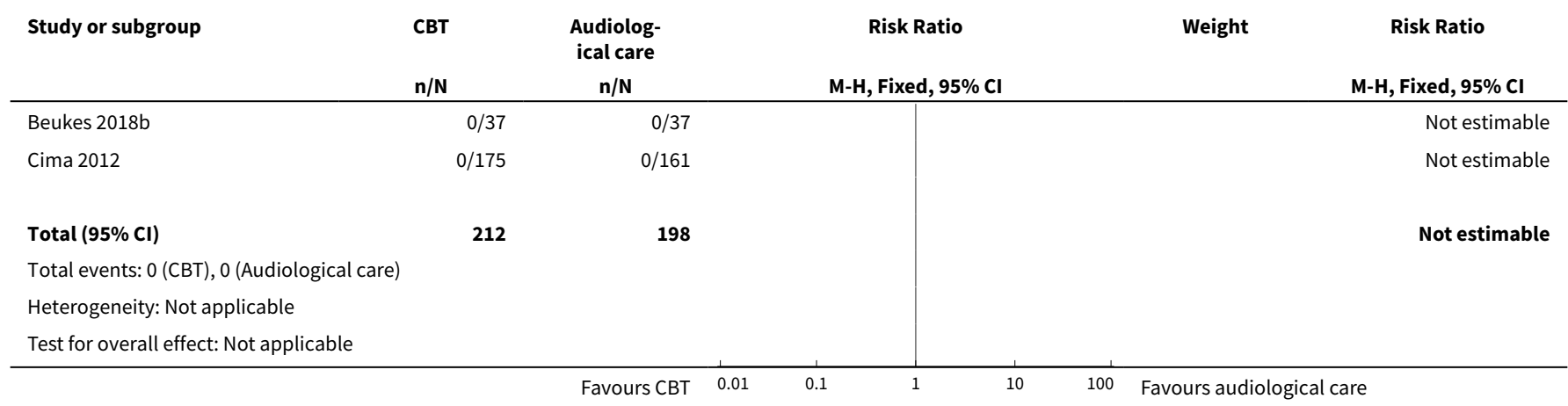

Analysis 2.3. Comparison 2 CBT versus audiological care (tinnitus education and rehabilitation for hearing loss), Outcome 3 Depression at end of treatment.

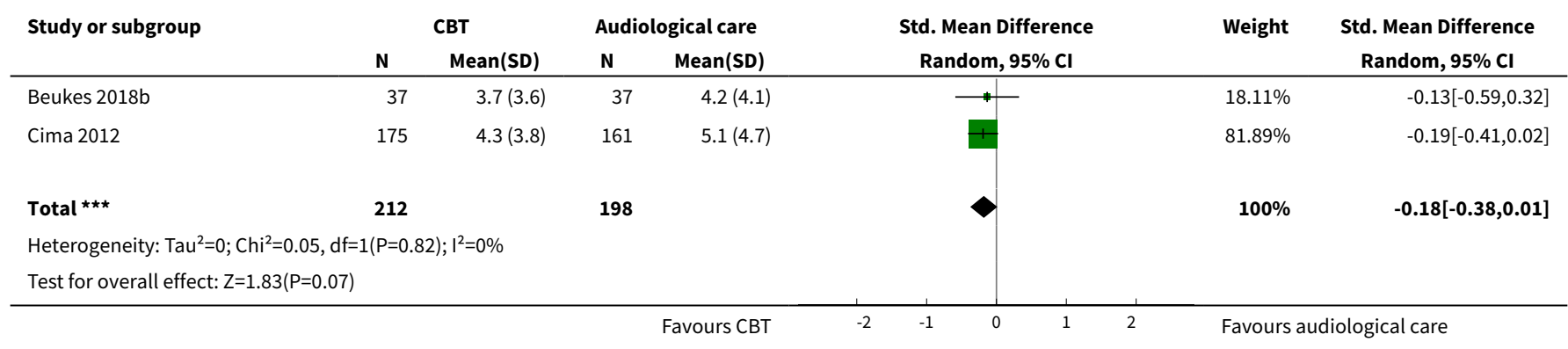


Analysis 2.4. Comparison 2 CBT versus audiological care (tinnitus education and rehabilitation for hearing loss), Outcome 4 Anxiety at end of treatment.

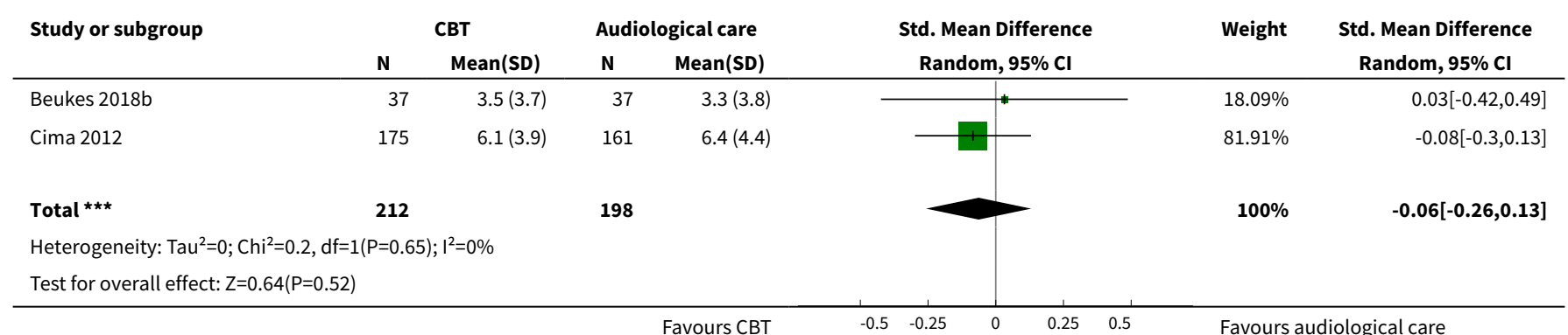

Analysis 2.5. Comparison 2 CBT versus audiological care (tinnitus education and rehabilitation for hearing loss), Outcome 5 Health-related quality of life.

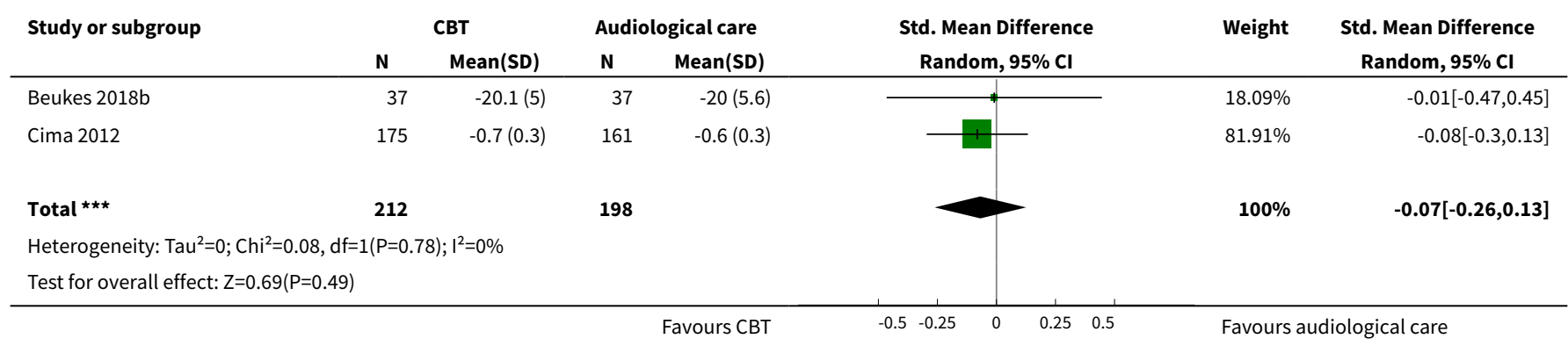

Analysis 2.6. Comparison 2 CBT versus audiological care (tinnitus education and rehabilitation for hearing loss), Outcome 6 Negatively biased interpretations of tinnitus.

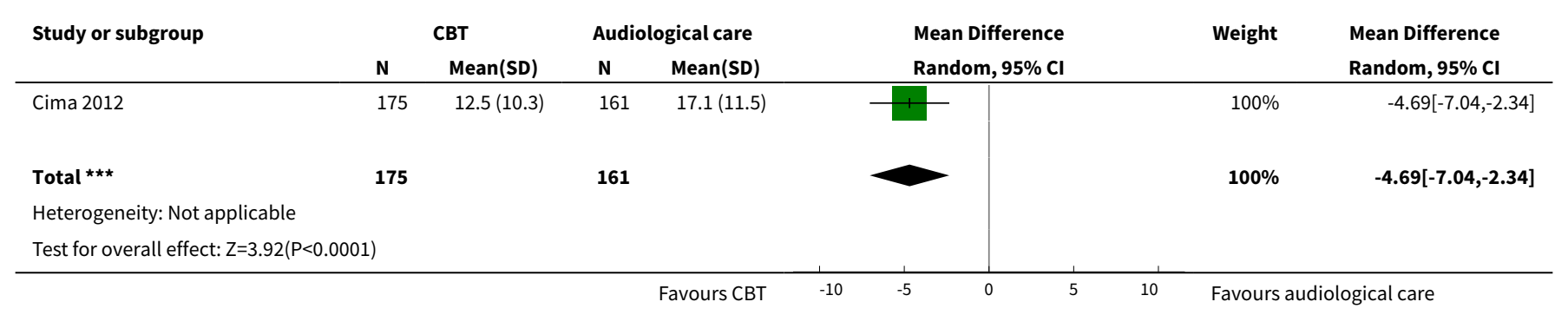

Analysis 2.7. Comparison 2 CBT versus audiological care (tinnitus education and rehabilitation for hearing loss), Outcome 7 Sensitivity analysis (fixed-effect model): impact of tinnitus on quality of life.

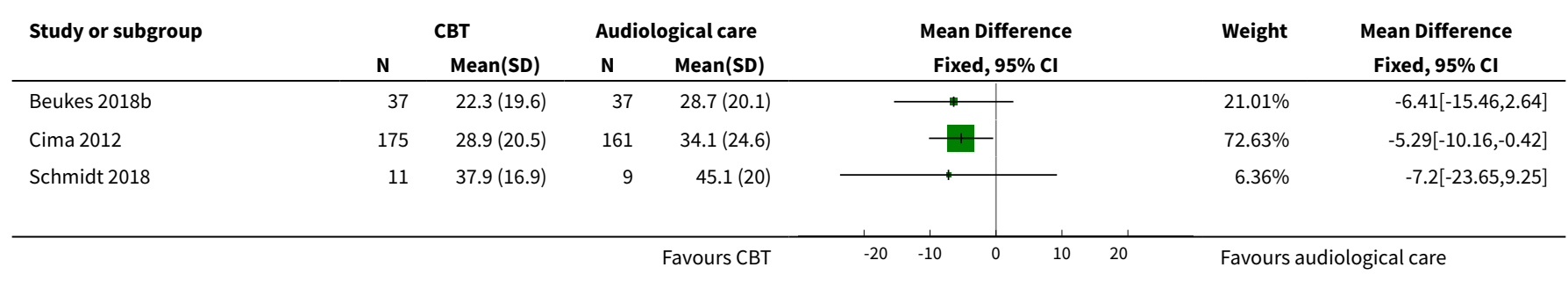




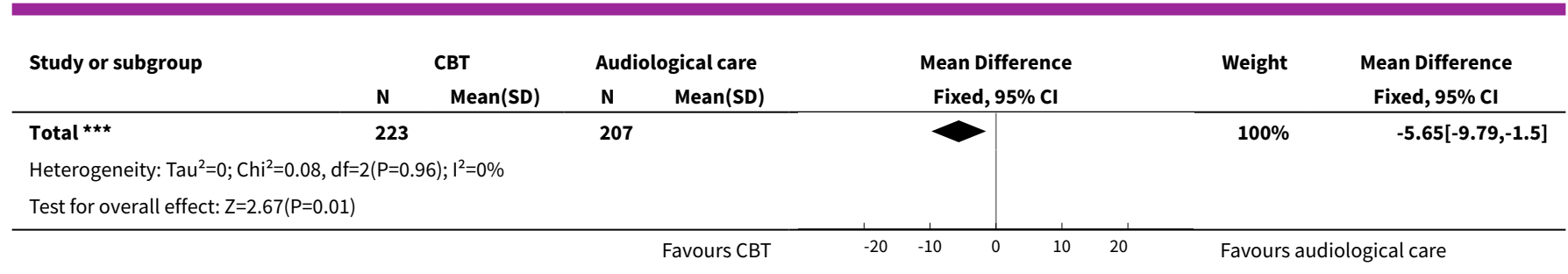

Comparison 3. CBT versus TRT (directive counselling and bilateral masking)

\begin{tabular}{|c|c|c|c|c|}
\hline Outcome or subgroup title & No. of studies & $\begin{array}{l}\text { No. of partici- } \\
\text { pants }\end{array}$ & Statistical method & Effect size \\
\hline $\begin{array}{l}1 \text { Impact of tinnitus on quality of life at } \\
\text { end of treatment }\end{array}$ & 1 & 42 & $\begin{array}{l}\text { Mean Difference (IV, Ran- } \\
\text { dom, } 95 \% \mathrm{CI} \text { ) }\end{array}$ & $\begin{array}{l}-15.79[-27.91 \\
-3.67]\end{array}$ \\
\hline $\begin{array}{l}2 \text { Impact of tinnitus on quality of life at } 6 \\
\text { months follow-up }\end{array}$ & 1 & 42 & $\begin{array}{l}\text { Mean Difference (IV, Ran- } \\
\text { dom, } 95 \% \mathrm{CI} \text { ) }\end{array}$ & $\begin{array}{l}-13.10[-26.08 \\
-0.12]\end{array}$ \\
\hline $\begin{array}{l}3 \text { Serious adverse effects at end of treat- } \\
\text { ment }\end{array}$ & 1 & 42 & $\begin{array}{l}\text { Risk Ratio (IV, Random, } \\
95 \% \mathrm{CI} \text { ) }\end{array}$ & $0.45[0.04,4.64]$ \\
\hline $\begin{array}{l}4 \text { Negatively biased interpretations of tin- } \\
\text { nitus at end of treatment }\end{array}$ & 1 & 42 & $\begin{array}{l}\text { Mean Difference (IV, Ran- } \\
\text { dom, } 95 \% \mathrm{CI} \text { ) }\end{array}$ & $\begin{array}{l}-9.78[-16.40 \\
-3.16]\end{array}$ \\
\hline $\begin{array}{l}5 \text { Negatively biased interpretations of tin- } \\
\text { nitus at } 6 \text { months follow-up }\end{array}$ & 1 & 42 & $\begin{array}{l}\text { Mean Difference (IV, Ran- } \\
\text { dom, } 95 \% \mathrm{CI} \text { ) }\end{array}$ & $\begin{array}{l}-8.28[-15.34 \\
-1.22]\end{array}$ \\
\hline
\end{tabular}

Analysis 3.1. Comparison 3 CBT versus TRT (directive counselling and bilateral masking), Outcome 1 Impact of tinnitus on quality of life at end of treatment.

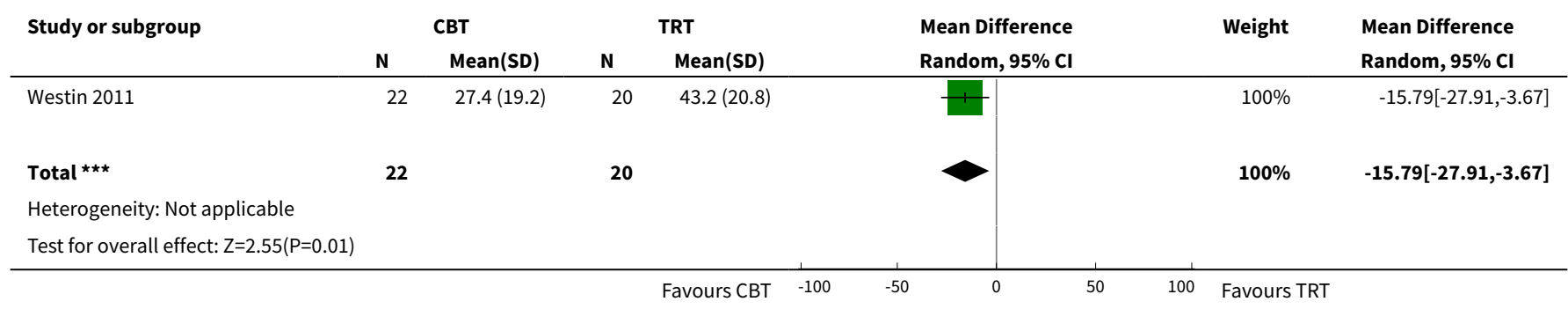

Analysis 3.2. Comparison 3 CBT versus TRT (directive counselling and bilateral masking), Outcome 2 Impact of tinnitus on quality of life at 6 months follow-up.

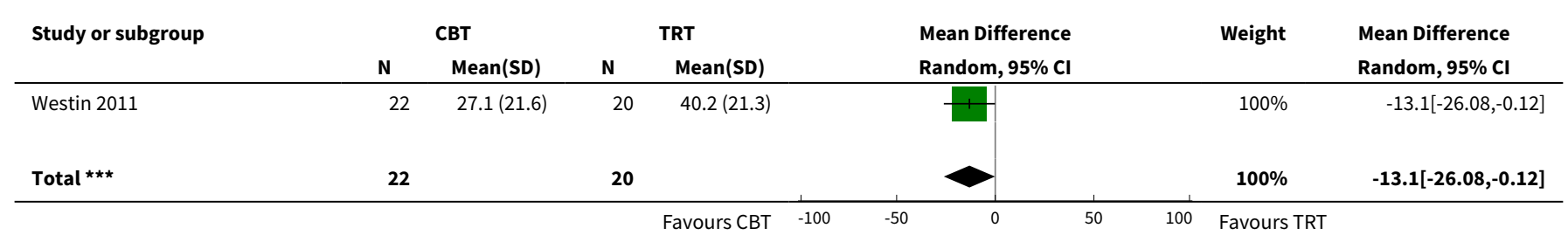




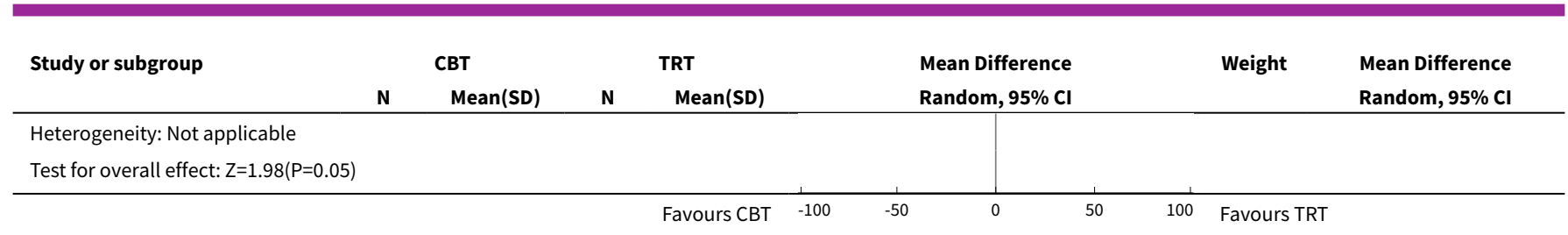

Analysis 3.3. Comparison 3 CBT versus TRT (directive counselling and bilateral masking), Outcome 3 Serious adverse effects at end of treatment.

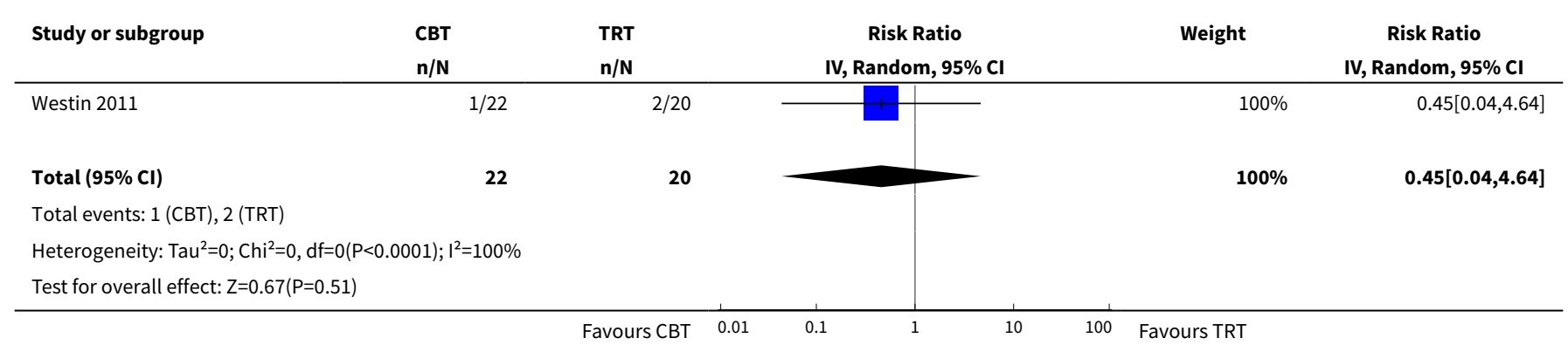

Analysis 3.4. Comparison 3 CBT versus TRT (directive counselling and bilateral masking), Outcome 4 Negatively biased interpretations of tinnitus at end of treatment.

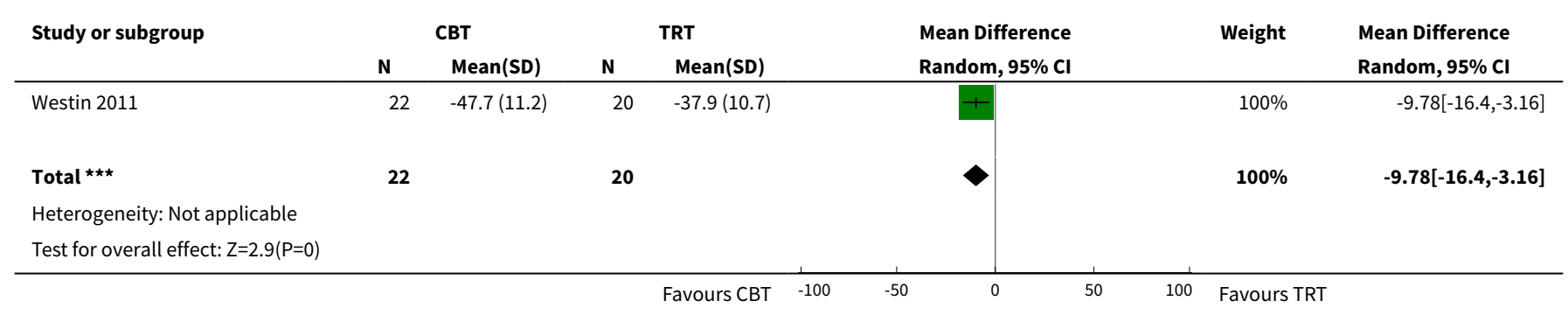

Analysis 3.5. Comparison 3 CBT versus TRT (directive counselling and bilateral masking), Outcome 5 Negatively biased interpretations of tinnitus at 6 months follow-up.

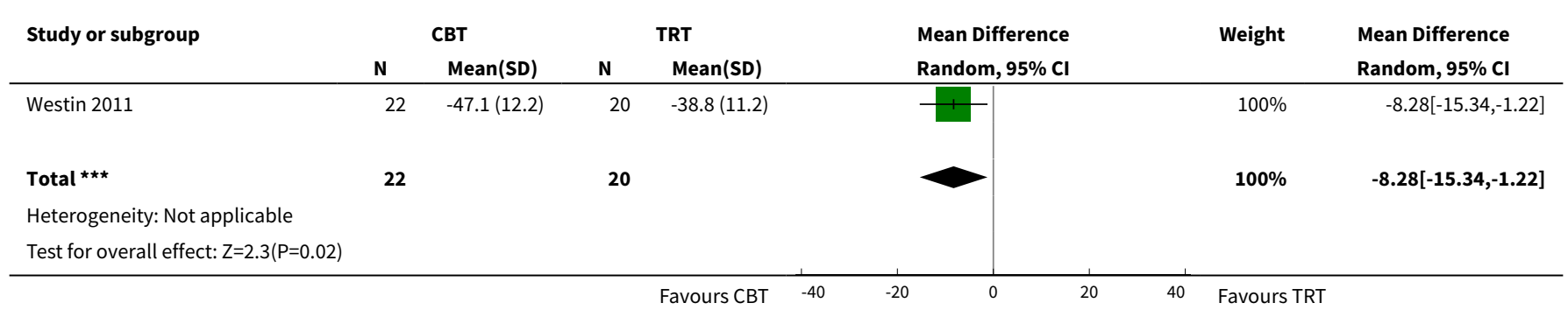


Comparison 4. CBT versus other active control

\begin{tabular}{|c|c|c|c|c|}
\hline Outcome or subgroup title & No. of studies & $\begin{array}{l}\text { No. of partici- } \\
\text { pants }\end{array}$ & Statistical method & Effect size \\
\hline $\begin{array}{l}1 \text { Impact of tinnitus on quality of life } \\
\text { at end of treatment }\end{array}$ & 12 & 966 & $\begin{array}{l}\text { Std. Mean Difference (IV, Ran- } \\
\text { dom, } 95 \% \mathrm{CI} \text { ) }\end{array}$ & $-0.30[-0.55,-0.05]$ \\
\hline $\begin{array}{l}2 \text { Impact of tinnitus on quality of life } \\
\text { at } 6 \text { months follow-up }\end{array}$ & 1 & 62 & $\begin{array}{l}\text { Mean Difference (IV, Random, } \\
95 \% \mathrm{CI} \text { ) }\end{array}$ & $\begin{array}{l}-11.80[-23.06 \\
-0.54]\end{array}$ \\
\hline $\begin{array}{l}3 \text { Impact of tinnitus on quality of life } \\
\text { at } 12 \text { months follow-up }\end{array}$ & 1 & 33 & $\begin{array}{l}\text { Mean Difference (IV, Random, } \\
95 \% \mathrm{CI} \text { ) }\end{array}$ & $-2.76[-14.69,9.17]$ \\
\hline $\begin{array}{l}4 \text { Serious adverse effects at the end } \\
\text { of treatment }\end{array}$ & 6 & 595 & $\begin{array}{l}\text { Risk Ratio (M-H, Random, 95\% } \\
\mathrm{Cl} \text { ) }\end{array}$ & $1.70[0.16,18.36]$ \\
\hline 5 Depression at end of treatment & 11 & 943 & $\begin{array}{l}\text { Std. Mean Difference (IV, Ran- } \\
\text { dom, } 95 \% \mathrm{CI} \text { ) }\end{array}$ & $-0.17[-0.33,-0.01]$ \\
\hline 6 Depression at 6 months follow-up & 1 & 62 & $\begin{array}{l}\text { Mean Difference (IV, Random, } \\
95 \% \mathrm{CI} \text { ) }\end{array}$ & $-1.90[-3.87,0.07]$ \\
\hline 7 Depression at 12 months & 1 & 33 & $\begin{array}{l}\text { Mean Difference (IV, Random, } \\
95 \% \mathrm{CI})\end{array}$ & $-2.0[-7.88,3.88]$ \\
\hline 8 Anxiety at end of treatment & 9 & 696 & $\begin{array}{l}\text { Std. Mean Difference (IV, Ran- } \\
\text { dom, } 95 \% \mathrm{CI} \text { ) }\end{array}$ & $-0.25[-0.48,-0.02]$ \\
\hline 9 Anxiety at 6 months follow-up & 1 & 62 & $\begin{array}{l}\text { Mean Difference (IV, Random, } \\
95 \% \mathrm{CI} \text { ) }\end{array}$ & $-1.20[-3.07,0.67]$ \\
\hline $\begin{array}{l}10 \text { Health-related quality of life at } \\
\text { end of treatment }\end{array}$ & 1 & 95 & $\begin{array}{l}\text { Mean Difference (IV, Random, } \\
95 \% \mathrm{CI} \text { ) }\end{array}$ & $-0.05[-0.68,0.59]$ \\
\hline $\begin{array}{l}11 \text { Negatively biased interpretations } \\
\text { of tinnitus at end of treatment }\end{array}$ & 5 & 455 & $\begin{array}{l}\text { Std. Mean Difference (IV, Ran- } \\
\text { dom, } 95 \% \mathrm{CI} \text { ) }\end{array}$ & $-0.55[-0.75,-0.35]$ \\
\hline $\begin{array}{l}12 \text { Negatively biased interpretations } \\
\text { of tinnitus at } 6 \text { months follow-up }\end{array}$ & 1 & 62 & $\begin{array}{l}\text { Mean Difference (IV, Random, } \\
95 \% \mathrm{CI} \text { ) }\end{array}$ & $\begin{array}{l}-7.20[-13.65 \\
-0.75]\end{array}$ \\
\hline $\begin{array}{l}13 \text { Negatively biased interpretations } \\
\text { of tinnitus at } 12 \text { months follow-up }\end{array}$ & 1 & 33 & $\begin{array}{l}\text { Mean Difference (IV, Random, } \\
95 \% \mathrm{CI} \text { ) }\end{array}$ & $\begin{array}{l}-14.05[-24.80, \\
-3.30]\end{array}$ \\
\hline $\begin{array}{l}14 \text { Subgroup analysis (random-ef- } \\
\text { fects model): type of therapy - im- } \\
\text { pact of tinnitus on quality of life }\end{array}$ & 12 & 966 & $\begin{array}{l}\text { Std. Mean Difference (IV, Ran- } \\
\text { dom, } 95 \% \mathrm{CI} \text { ) }\end{array}$ & $-0.30[-0.55,-0.05]$ \\
\hline 14.1 CBT & 5 & 626 & $\begin{array}{l}\text { Std. Mean Difference (IV, Ran- } \\
\text { dom, } 95 \% \mathrm{CI} \text { ) }\end{array}$ & $-0.23[-0.62,0.15]$ \\
\hline 14.2 Cognitive therapy & 3 & 97 & $\begin{array}{l}\text { Std. Mean Difference (IV, Ran- } \\
\text { dom, } 95 \% \mathrm{CI} \text { ) }\end{array}$ & $-0.38[-0.92,0.17]$ \\
\hline $14.3 \mathrm{ACT}$ & 2 & 114 & $\begin{array}{l}\text { Std. Mean Difference (IV, Ran- } \\
\text { dom, } 95 \% \mathrm{CI} \text { ) }\end{array}$ & $-0.20[-1.60,1.21]$ \\
\hline 14.4 Mindfulness & 2 & 129 & $\begin{array}{l}\text { Std. Mean Difference (IV, Ran- } \\
\text { dom, } 95 \% \mathrm{CI} \text { ) }\end{array}$ & $-0.35[-0.70,-0.00]$ \\
\hline
\end{tabular}




\begin{tabular}{|c|c|c|c|c|}
\hline Outcome or subgroup title & No. of studies & $\begin{array}{l}\text { No. of partici- } \\
\text { pants }\end{array}$ & Statistical method & Effect size \\
\hline $\begin{array}{l}15 \text { Subgroup analysis (fixed-effect } \\
\text { model): type of therapy - impact of } \\
\text { tinnitus on quality of life }\end{array}$ & 12 & 966 & $\begin{array}{l}\text { Std. Mean Difference (IV, Fixed, } \\
95 \% \mathrm{CI})\end{array}$ & $-0.33[-0.46,-0.19]$ \\
\hline $15.1 \mathrm{CBT}$ & 5 & 626 & $\begin{array}{l}\text { Std. Mean Difference (IV, Fixed, } \\
95 \% \mathrm{CI} \text { ) }\end{array}$ & $-0.27[-0.44,-0.10]$ \\
\hline 15.2 Cognitive therapy & 3 & 97 & $\begin{array}{l}\text { Std. Mean Difference (IV, Fixed, } \\
95 \% \mathrm{CI} \text { ) }\end{array}$ & $-0.40[-0.82,0.02]$ \\
\hline $15.3 \mathrm{ACT}$ & 2 & 114 & $\begin{array}{l}\text { Std. Mean Difference (IV, Fixed, } \\
95 \% \mathrm{CI} \text { ) }\end{array}$ & $-0.59[-0.99,-0.19]$ \\
\hline 15.4 Mindfulness & 2 & 129 & $\begin{array}{l}\text { Std. Mean Difference (IV, Fixed, } \\
95 \% \mathrm{CI} \text { ) }\end{array}$ & $-0.35[-0.70,-0.00]$ \\
\hline $\begin{array}{l}16 \text { Subgroup analysis: mode of de- } \\
\text { livery - impact of tinnitus on quality } \\
\text { of life }\end{array}$ & 12 & 966 & $\begin{array}{l}\text { Std. Mean Difference (IV, Ran- } \\
\text { dom, 95\% CI) }\end{array}$ & $-0.31[-0.55,-0.08]$ \\
\hline 16.1 Face-to-face & 9 & 633 & $\begin{array}{l}\text { Std. Mean Difference (IV, Ran- } \\
\text { dom, 95\% CI) }\end{array}$ & $-0.20[-0.43,0.03]$ \\
\hline 16.2 Internet-based CBT & 4 & 333 & $\begin{array}{l}\text { Std. Mean Difference (IV, Ran- } \\
\text { dom, } 95 \% \mathrm{CI} \text { ) }\end{array}$ & $-0.52[-0.98,-0.07]$ \\
\hline $\begin{array}{l}17 \text { Subgroup analysis: unit of deliv- } \\
\text { ery - impact of tinnitus on quality of } \\
\text { life }\end{array}$ & 12 & 966 & $\begin{array}{l}\text { Std. Mean Difference (IV, Ran- } \\
\text { dom, } 95 \% \mathrm{CI} \text { ) }\end{array}$ & $-0.31[-0.55,-0.08]$ \\
\hline 17.1 Individual & 6 & 424 & $\begin{array}{l}\text { Std. Mean Difference (IV, Ran- } \\
\text { dom, } 95 \% \mathrm{CI} \text { ) }\end{array}$ & $-0.45[-0.79,-0.11]$ \\
\hline 17.2 Group & 7 & 542 & $\begin{array}{l}\text { Std. Mean Difference (IV, Ran- } \\
\text { dom, } 95 \% \mathrm{CI} \text { ) }\end{array}$ & $-0.19[-0.48,0.10]$ \\
\hline $\begin{array}{l}18 \text { Subgroup analysis: who delivers } \\
\text { intervention - impact of tinnitus on } \\
\text { quality of life }\end{array}$ & 12 & 966 & $\begin{array}{l}\text { Std. Mean Difference (IV, Ran- } \\
\text { dom, } 95 \% \mathrm{CI} \text { ) }\end{array}$ & $-0.31[-0.55,-0.08]$ \\
\hline 18.1 Psychologists & 8 & 669 & $\begin{array}{l}\text { Std. Mean Difference (IV, Ran- } \\
\text { dom, } 95 \% \mathrm{Cl} \text { ) }\end{array}$ & $-0.29[-0.61,0.02]$ \\
\hline 18.2 Internet-based & 3 & 209 & $\begin{array}{l}\text { Std. Mean Difference (IV, Ran- } \\
\text { dom, } 95 \% \mathrm{CI} \text { ) }\end{array}$ & $-0.40[-1.03,0.23]$ \\
\hline 18.3 Other 'therapists' & 2 & 88 & $\begin{array}{l}\text { Std. Mean Difference (IV, Ran- } \\
\text { dom, } 95 \% \mathrm{CI} \text { ) }\end{array}$ & $-0.25[-0.68,0.18]$ \\
\hline $\begin{array}{l}19 \text { Subgroup analysis: type of con- } \\
\text { trol - impact of tinnitus on quality of } \\
\text { life }\end{array}$ & 12 & 965 & $\begin{array}{l}\text { Std. Mean Difference (IV, Ran- } \\
\text { dom, } 95 \% \mathrm{CI} \text { ) }\end{array}$ & $-0.30[-0.54,-0.06]$ \\
\hline 19.1 Information & 3 & 171 & $\begin{array}{l}\text { Std. Mean Difference (IV, Ran- } \\
\text { dom, } 95 \% \mathrm{Cl} \text { ) }\end{array}$ & $-0.32[-0.91,0.27]$ \\
\hline
\end{tabular}




\begin{tabular}{|c|c|c|c|c|}
\hline Outcome or subgroup title & No. of studies & $\begin{array}{l}\text { No. of partici- } \\
\text { pants }\end{array}$ & Statistical method & Effect size \\
\hline 19.2 Coping effectiveness training & 1 & 19 & $\begin{array}{l}\text { Std. Mean Difference (IV, Ran- } \\
\text { dom, } 95 \% \mathrm{CI} \text { ) }\end{array}$ & $0.58[-0.34,1.51]$ \\
\hline 19.3 Relaxation & 3 & 159 & $\begin{array}{l}\text { Std. Mean Difference (IV, Ran- } \\
\text { dom, } 95 \% \mathrm{CI} \text { ) }\end{array}$ & $-0.31[-0.63,0.01]$ \\
\hline 19.4 Discussion forum & 3 & 347 & $\begin{array}{l}\text { Std. Mean Difference (IV, Ran- } \\
\text { dom, } 95 \% \mathrm{CI} \text { ) }\end{array}$ & $-0.72[-0.95,-0.49]$ \\
\hline 19.5 Masking & 1 & 27 & $\begin{array}{l}\text { Std. Mean Difference (IV, Ran- } \\
\text { dom, } 95 \% \mathrm{CI} \text { ) }\end{array}$ & $-0.03[-0.83,0.77]$ \\
\hline 19.6 Virtual reality & 1 & 116 & $\begin{array}{l}\text { Std. Mean Difference (IV, Ran- } \\
\text { dom, } 95 \% \mathrm{CI} \text { ) }\end{array}$ & $0.19[-0.18,0.56]$ \\
\hline 19.7 Self-help & 1 & 126 & $\begin{array}{l}\text { Std. Mean Difference (IV, Ran- } \\
\text { dom, } 95 \% \mathrm{CI} \text { ) }\end{array}$ & $-0.06[-0.52,0.39]$ \\
\hline $\begin{array}{l}20 \text { Sensitivity analysis (fixed-effect } \\
\text { model): impact of tinnitus on quali- } \\
\text { ty of life at end of treatment }\end{array}$ & 12 & 966 & $\begin{array}{l}\text { Std. Mean Difference (IV, Fixed, } \\
95 \% \mathrm{CI} \text { ) }\end{array}$ & $-0.33[-0.46,-0.19]$ \\
\hline $\begin{array}{l}21 \text { Sensitivity analysis: without } \\
\text { studies at high risk of bias for in- } \\
\text { complete outcome data - impact of } \\
\text { tinnitus on quality of life }\end{array}$ & 9 & 770 & $\begin{array}{l}\text { Std. Mean Difference (IV, Ran- } \\
\text { dom, } 95 \% \mathrm{CI} \text { ) }\end{array}$ & $-0.48[-0.71,-0.26]$ \\
\hline $\begin{array}{l}22 \text { Sensitivity analysis: without high } \\
\text { risk of bias of missing outcome da- } \\
\text { ta, by subgroups (random-effects } \\
\text { model): type of therapy - impact of } \\
\text { tinnitus on quality of life }\end{array}$ & 9 & 769 & $\begin{array}{l}\text { Std. Mean Difference (IV, Ran- } \\
\text { dom, } 95 \% \mathrm{CI} \text { ) }\end{array}$ & $-0.45[-0.70,-0.21]$ \\
\hline $22.1 \mathrm{CBT}$ & 3 & 459 & $\begin{array}{l}\text { Std. Mean Difference (IV, Ran- } \\
\text { dom, } 95 \% \mathrm{CI} \text { ) }\end{array}$ & $-0.49[-0.87,-0.12]$ \\
\hline 22.2 Cognitive therapy & 2 & 67 & $\begin{array}{l}\text { Std. Mean Difference (IV, Ran- } \\
\text { dom, } 95 \% \mathrm{CI} \text { ) }\end{array}$ & $-0.48[-1.31,0.34]$ \\
\hline $22.3 \mathrm{ACT}$ & 2 & 114 & $\begin{array}{l}\text { Std. Mean Difference (IV, Ran- } \\
\text { dom, } 95 \% \mathrm{Cl} \text { ) }\end{array}$ & $-0.20[-1.60,1.21]$ \\
\hline 22.4 Mindfulness & 2 & 129 & $\begin{array}{l}\text { Std. Mean Difference (IV, Ran- } \\
\text { dom, } 95 \% \mathrm{CI} \text { ) }\end{array}$ & $-0.35[-0.70,-0.00]$ \\
\hline $\begin{array}{l}23 \text { Sensitivity analysis: optimistic } \\
\text { assumption for Malinvaud - impact } \\
\text { of tinnitus on quality of life }\end{array}$ & 12 & 955 & $\begin{array}{l}\text { Std. Mean Difference (IV, Ran- } \\
\text { dom, } 95 \% \mathrm{CI} \text { ) }\end{array}$ & $-0.35[-0.57,-0.13]$ \\
\hline $\begin{array}{l}24 \text { Sensitivity analysis: optimistic } \\
\text { assumption for Malinvaud - depres- } \\
\text { sion }\end{array}$ & 11 & 921 & $\begin{array}{l}\text { Std. Mean Difference (IV, Ran- } \\
\text { dom, } 95 \% \mathrm{CI} \text { ) }\end{array}$ & $-0.21[-0.34,-0.07]$ \\
\hline $\begin{array}{l}25 \text { Sensitivity analysis: optimistic } \\
\text { assumption for Malinvaud - anxiety }\end{array}$ & 9 & 674 & $\begin{array}{l}\text { Std. Mean Difference (IV, Ran- } \\
\text { dom, } 95 \% \mathrm{CI})\end{array}$ & $-0.28[-0.48,-0.09]$ \\
\hline
\end{tabular}


Analysis 4.1. Comparison 4 CBT versus other active control, Outcome 1 Impact of tinnitus on quality of life at end of treatment.

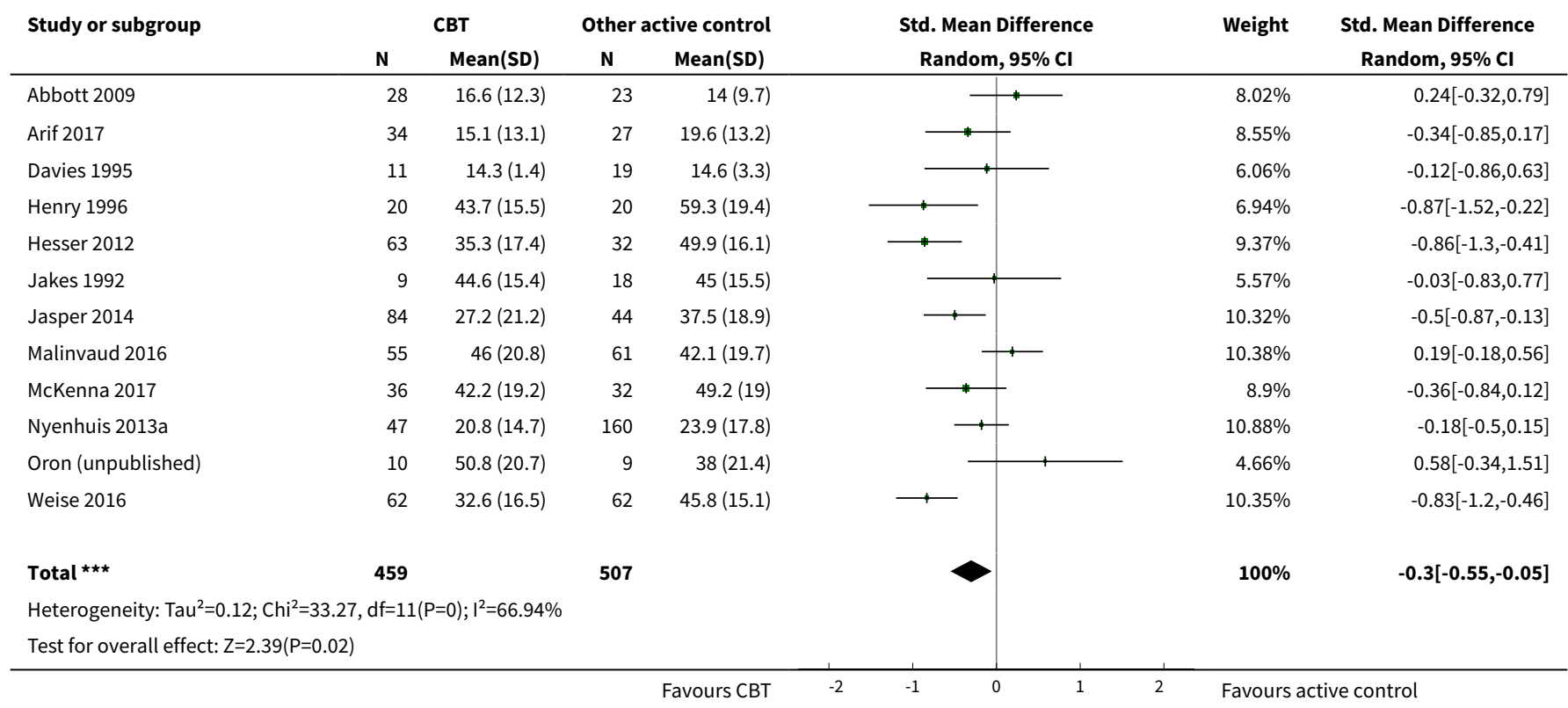

Analysis 4.2. Comparison 4 CBT versus other active control, Outcome 2 Impact of tinnitus on quality of life at 6 months follow-up.

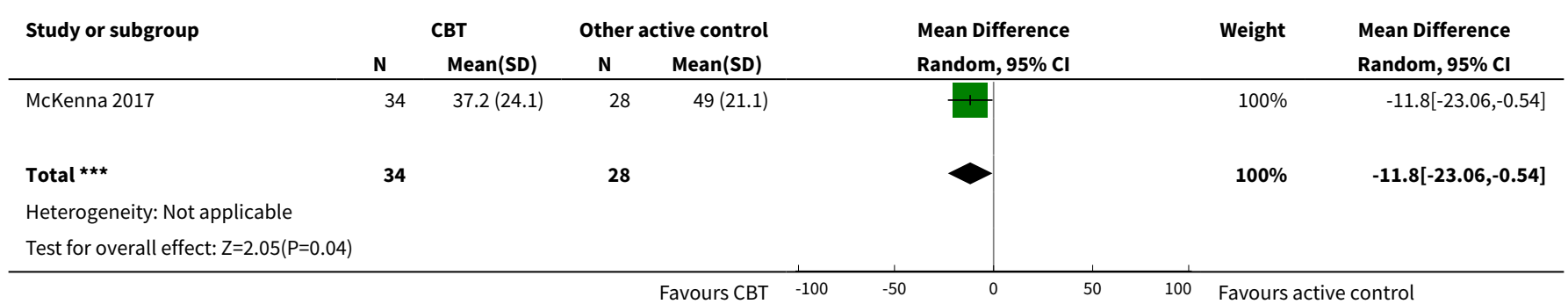

Analysis 4.3. Comparison $4 \mathrm{CBT}$ versus other active control, Outcome 3 Impact of tinnitus on quality of life at $\mathbf{1 2}$ months follow-up.

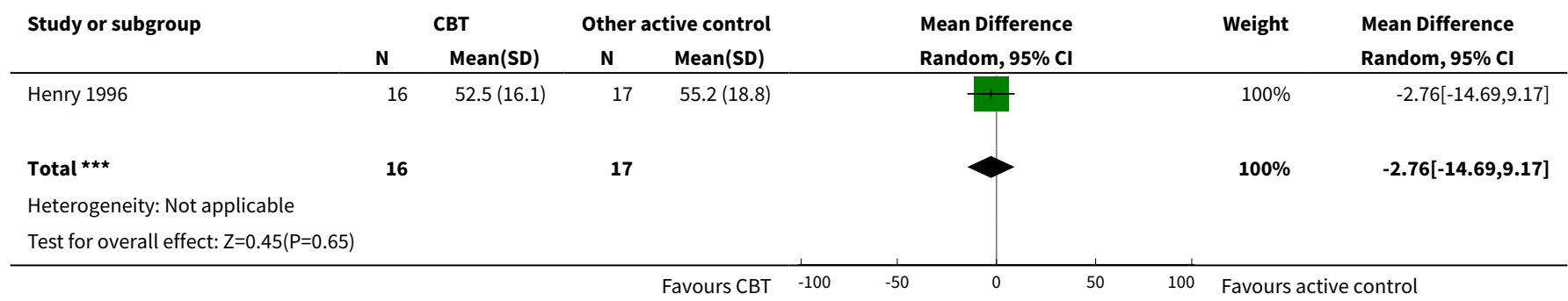


Analysis 4.4. Comparison 4 CBT versus other active control, Outcome 4 Serious adverse effects at the end of treatment.

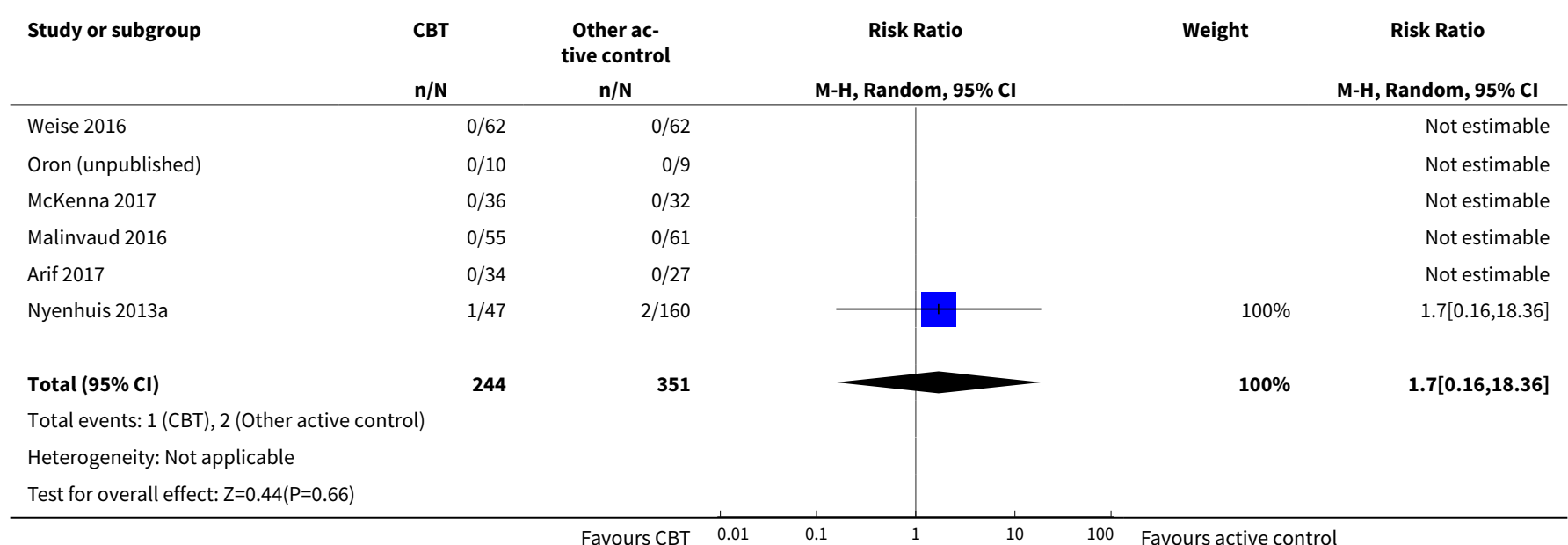

Analysis 4.5. Comparison 4 CBT versus other active control, Outcome 5 Depression at end of treatment.

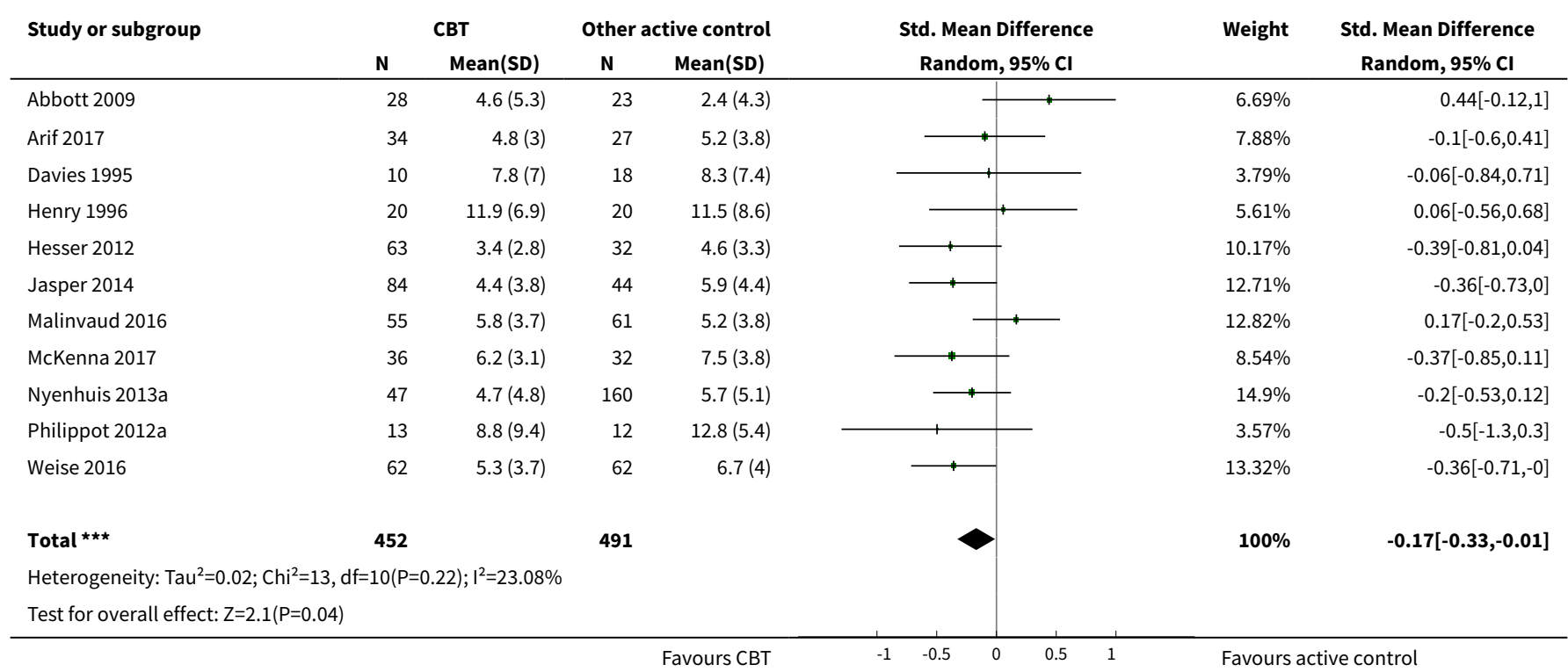

\section{Analysis 4.6. Comparison 4 CBT versus other active control, Outcome 6 Depression at 6 months follow-up.}

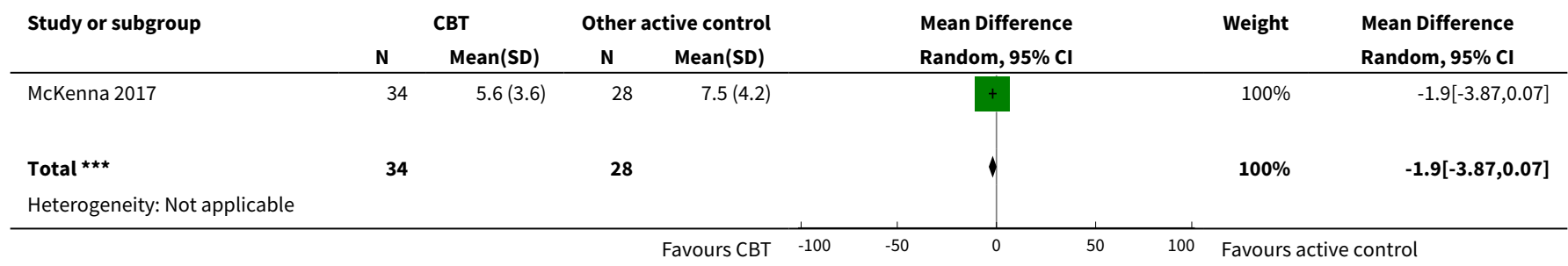




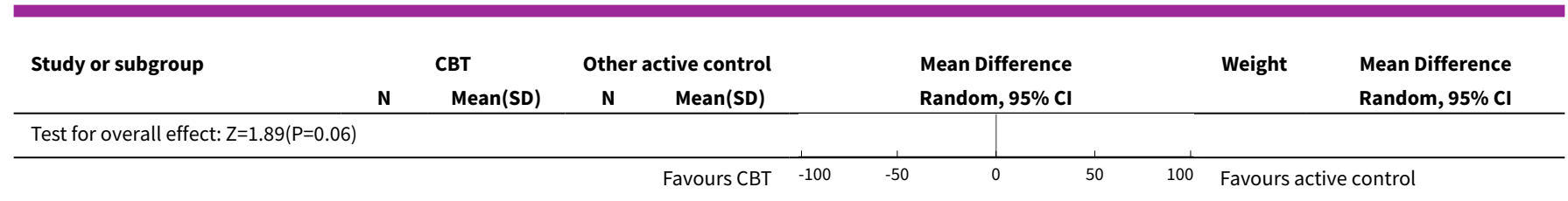

Analysis 4.7. Comparison 4 CBT versus other active control, Outcome 7 Depression at 12 months.

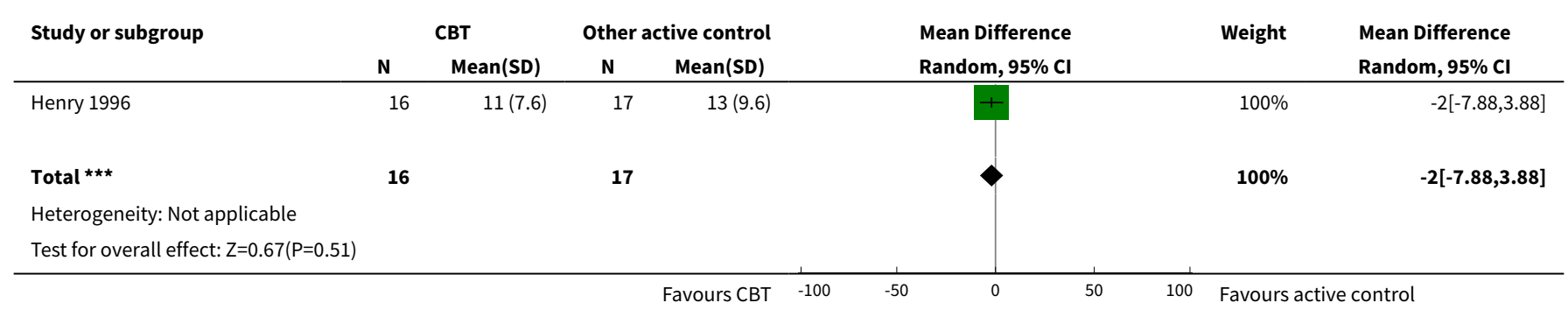

Analysis 4.8. Comparison 4 CBT versus other active control, Outcome 8 Anxiety at end of treatment.

\begin{tabular}{|c|c|c|c|c|c|c|c|}
\hline \multirow{3}{*}{$\begin{array}{l}\text { Study or subgroup } \\
\text { Abbott } 2009\end{array}$} & \multicolumn{2}{|c|}{ CBT } & \multicolumn{2}{|c|}{ Other active control } & \multirow{2}{*}{$\begin{array}{c}\text { Std. Mean Difference } \\
\text { Random, } 95 \% \mathrm{Cl}\end{array}$} & \multirow[t]{2}{*}{ Weight } & \multirow{2}{*}{$\begin{array}{c}\text { Std. Mean Difference } \\
\text { Random, } 95 \% \mathrm{Cl}\end{array}$} \\
\hline & $\mathbf{N}$ & $\operatorname{Mean}(S D)$ & $\mathbf{N}$ & Mean(SD) & & & \\
\hline & 28 & $3.8(3.8)$ & 23 & $3.1(4)$ & $\begin{array}{ll} \\
\end{array}$ & $9.77 \%$ & $0.18[-0.38,0.73]$ \\
\hline Arif 2017 & 34 & $4.6(2.8)$ & 27 & $5.9(4)$ & - & $10.68 \%$ & $-0.38[-0.89,0.13]$ \\
\hline Davies 1995 & 10 & $45.1(8.9)$ & 18 & $46.9(10.7)$ & & $6.31 \%$ & $-0.18[-0.95,0.6]$ \\
\hline Hesser 2012 & 63 & $4.4(2.8)$ & 32 & $6.8(4)$ & & $12.4 \%$ & $-0.72[-1.15,-0.28]$ \\
\hline Jasper 2014 & 84 & $5.6(3.5)$ & 44 & $7.7(4.7)$ & & $14.24 \%$ & $-0.51[-0.88,-0.14]$ \\
\hline McKenna 2017 & 36 & $9.2(3.8)$ & 32 & $10.1(3.9)$ & 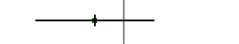 & $11.41 \%$ & $-0.23[-0.71,0.25]$ \\
\hline Philippot 2012a & 13 & $42.9(12.8)$ & 12 & $45.8(7.7)$ & & $6.15 \%$ & $-0.26[-1.05,0.53]$ \\
\hline Weise 2016 & 62 & $6.7(3.4)$ & 62 & $7.8(3.3)$ & & $14.68 \%$ & $-0.35[-0.71,0]$ \\
\hline 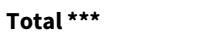 & 385 & & 311 & & & $100 \%$ & $-0.25[-0.48,-0.02]$ \\
\hline \multicolumn{8}{|c|}{ Heterogeneity: $\mathrm{Tau}^{2}=0.06 ; \mathrm{Chi}^{2}=16.54, \mathrm{df}=8(\mathrm{P}=0.04) ; \mathrm{I}^{2}=51.63 \%$} \\
\hline
\end{tabular}

Analysis 4.9. Comparison 4 CBT versus other active control, Outcome 9 Anxiety at 6 months follow-up.

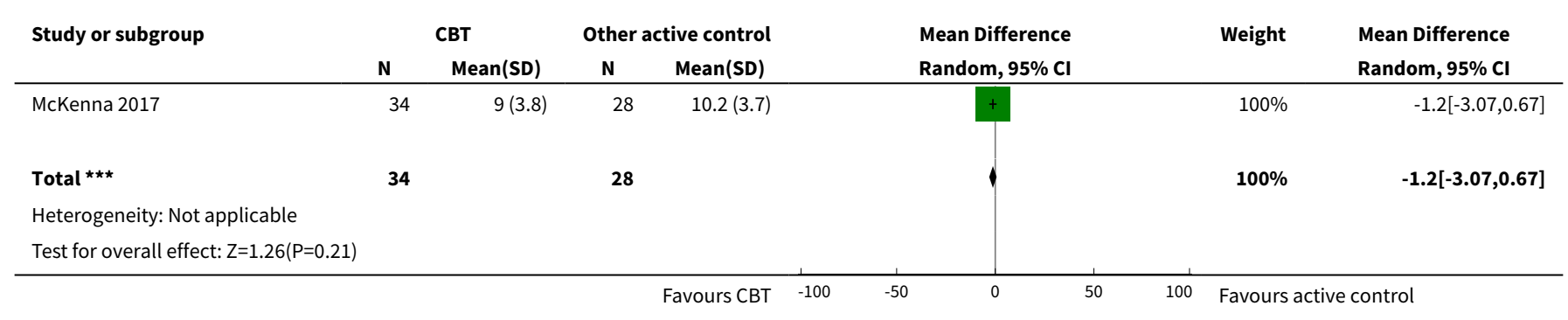


Analysis 4.10. Comparison 4 CBT versus other active control, Outcome 10 Health-related quality of life at end of treatment.

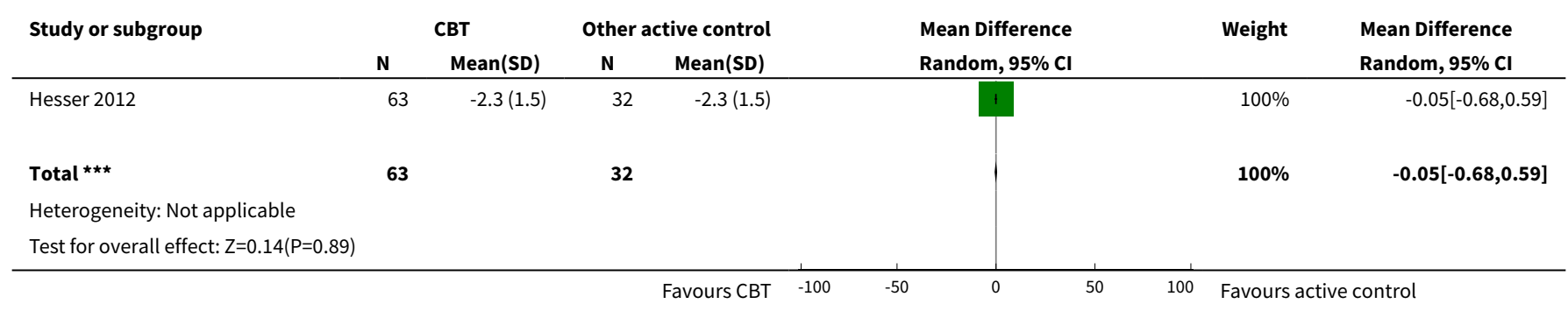

Analysis 4.11. Comparison 4 CBT versus other active control, Outcome 11 Negatively biased interpretations of tinnitus at end of treatment.

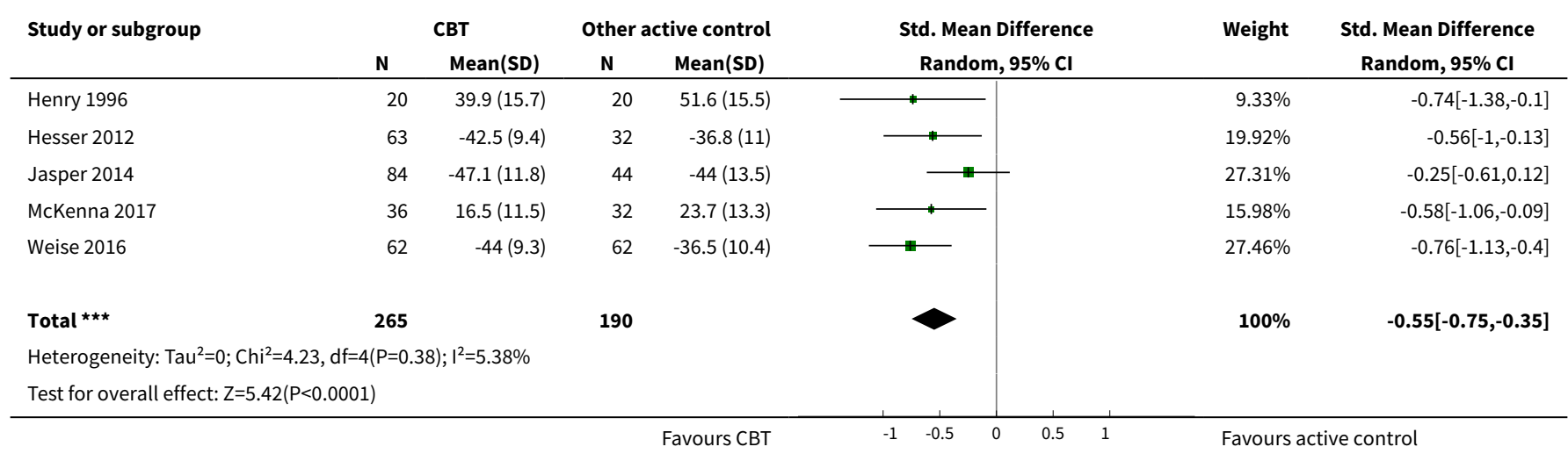

Analysis 4.12. Comparison 4 CBT versus other active control, Outcome 12 Negatively biased interpretations of tinnitus at 6 months follow-up.

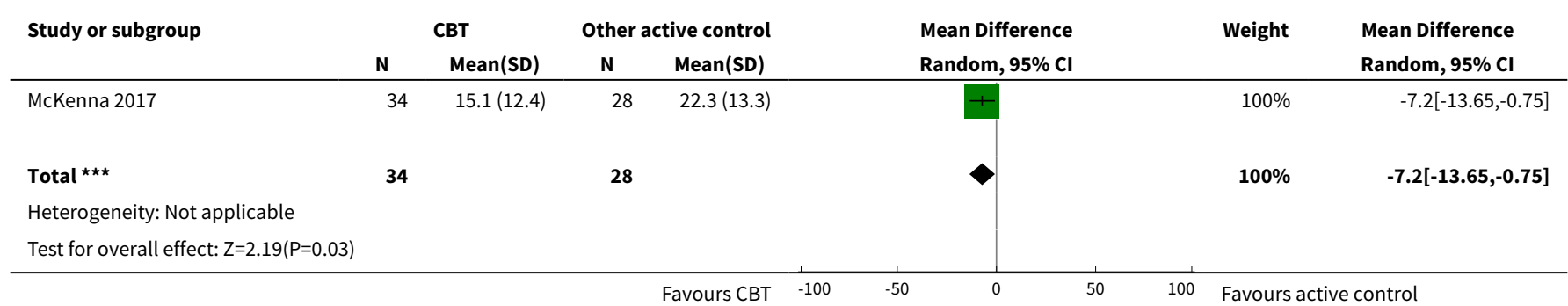

Analysis 4.13. Comparison 4 CBT versus other active control, Outcome 13 Negatively biased interpretations of tinnitus at 12 months follow-up.

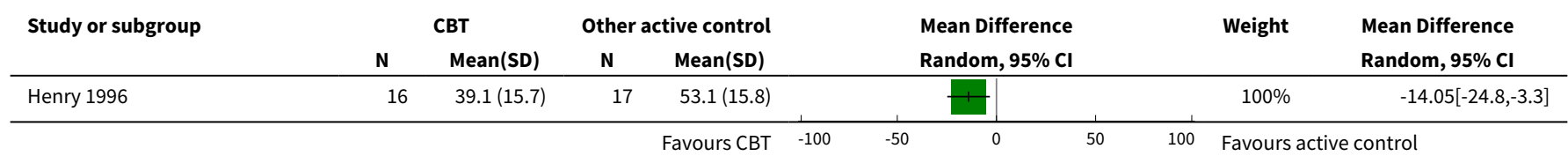




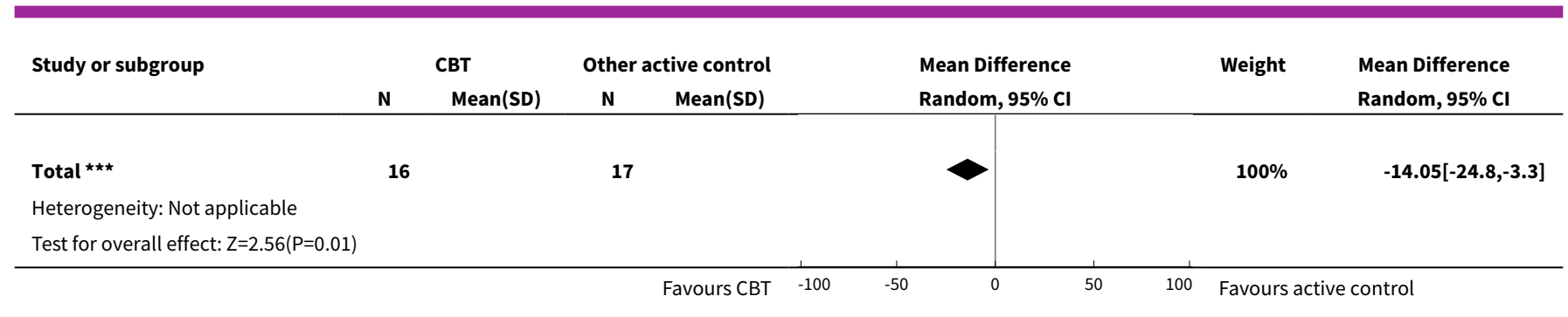

Analysis 4.14. Comparison 4 CBT versus other active control, Outcome 14 Subgroup analysis (random-effects model): type of therapy - impact of tinnitus on quality of life.

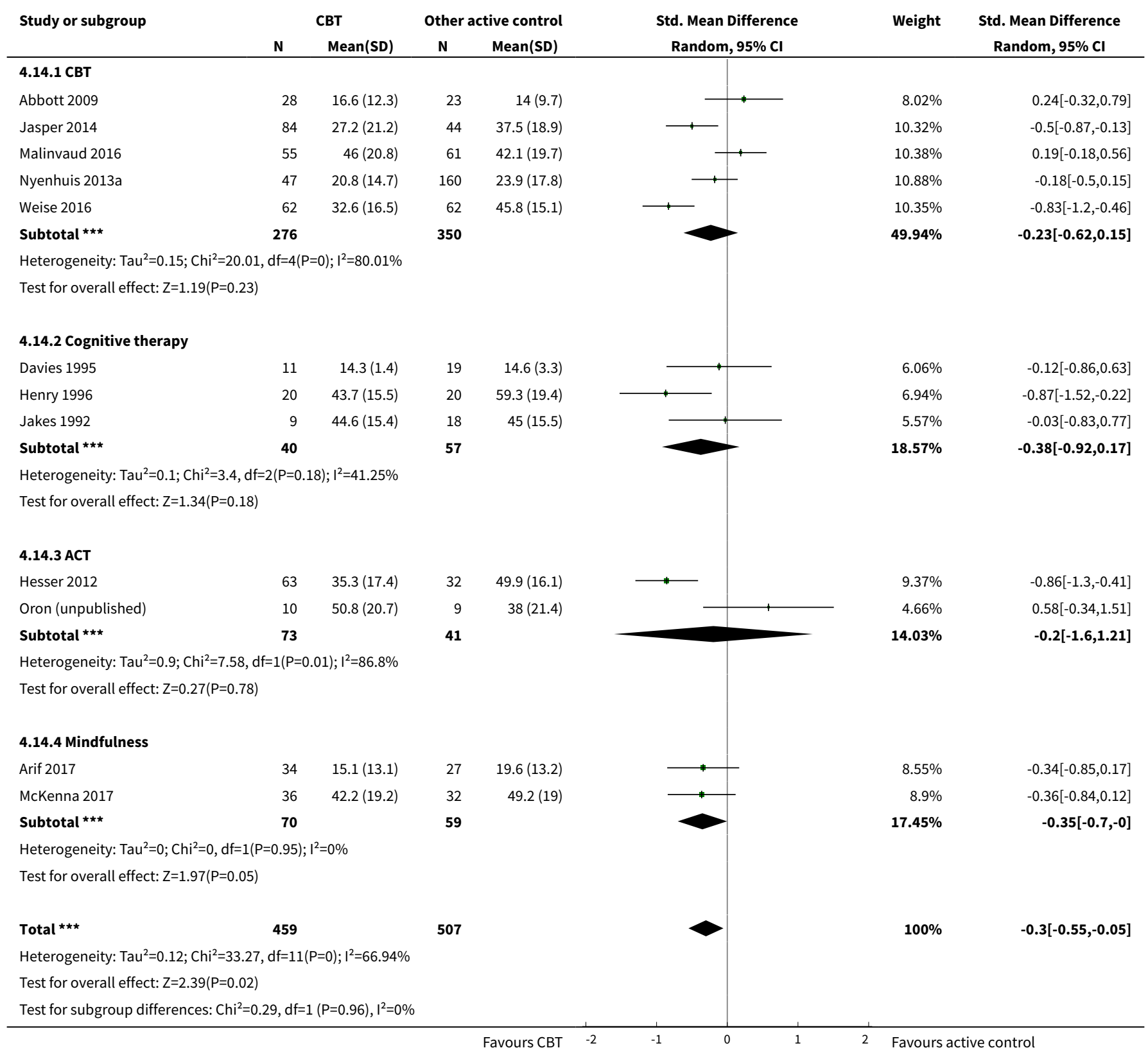


Analysis 4.15. Comparison 4 CBT versus other active control, Outcome 15 Subgroup analysis (fixed-effect model): type of therapy - impact of tinnitus on quality of life.

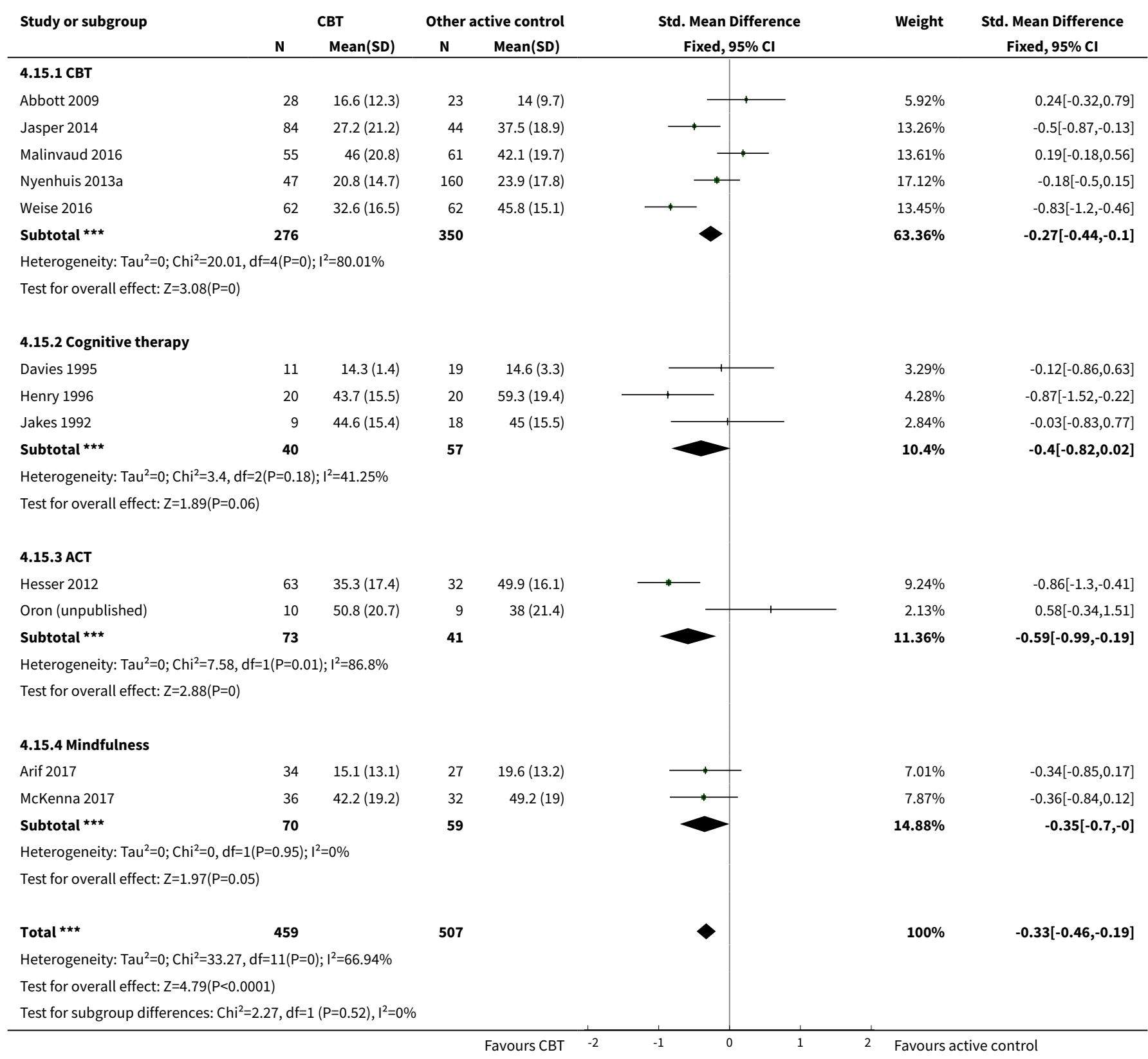

Analysis 4.16. Comparison 4 CBT versus other active control, Outcome 16 Subgroup analysis: mode of delivery - impact of tinnitus on quality of life.

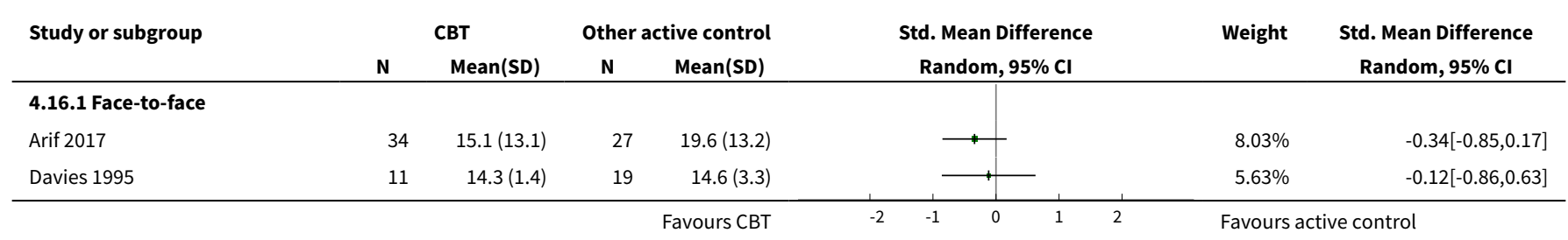




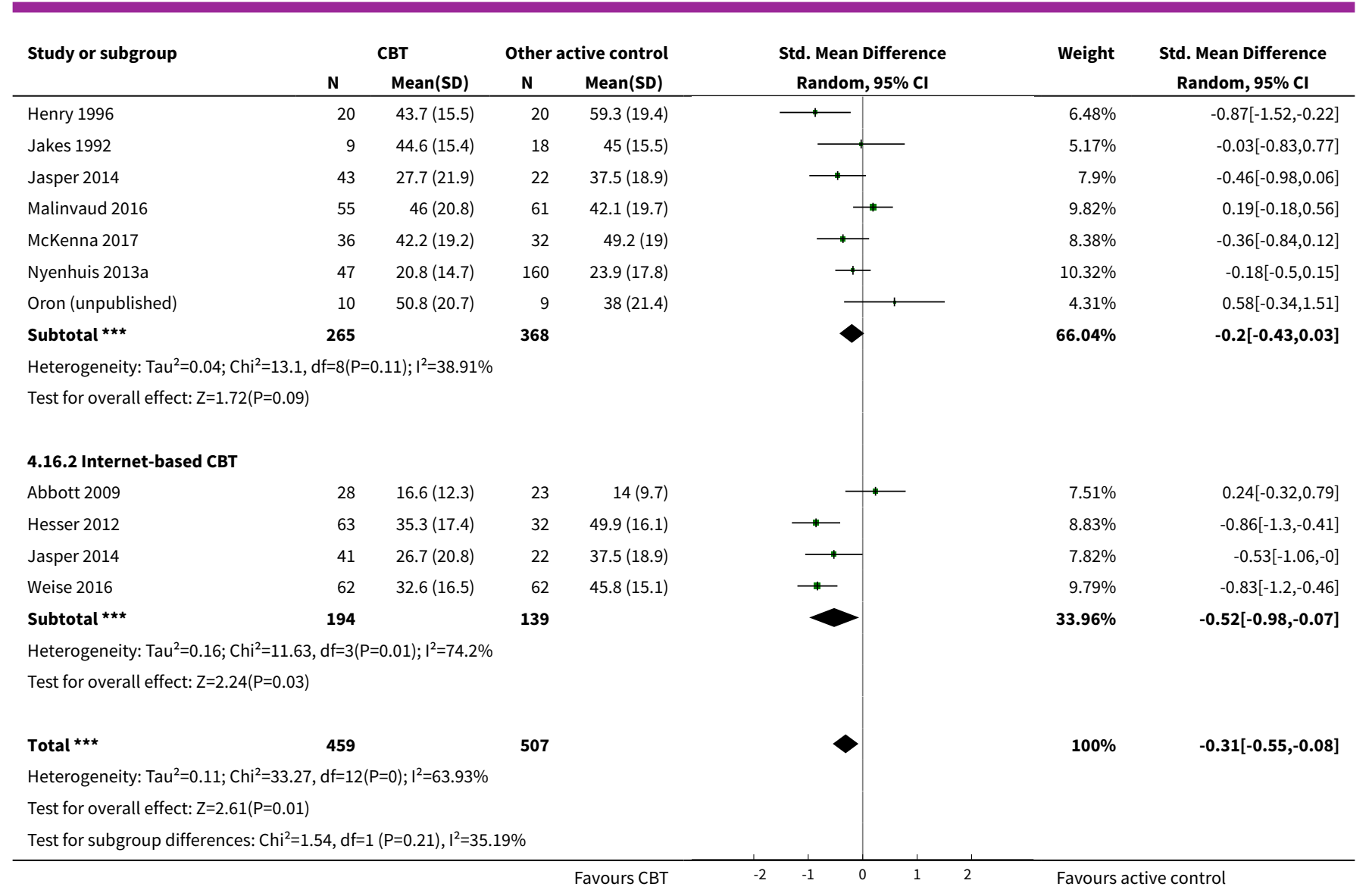

Analysis 4.17. Comparison 4 CBT versus other active control, Outcome 17 Subgroup analysis: unit of delivery - impact of tinnitus on quality of life.

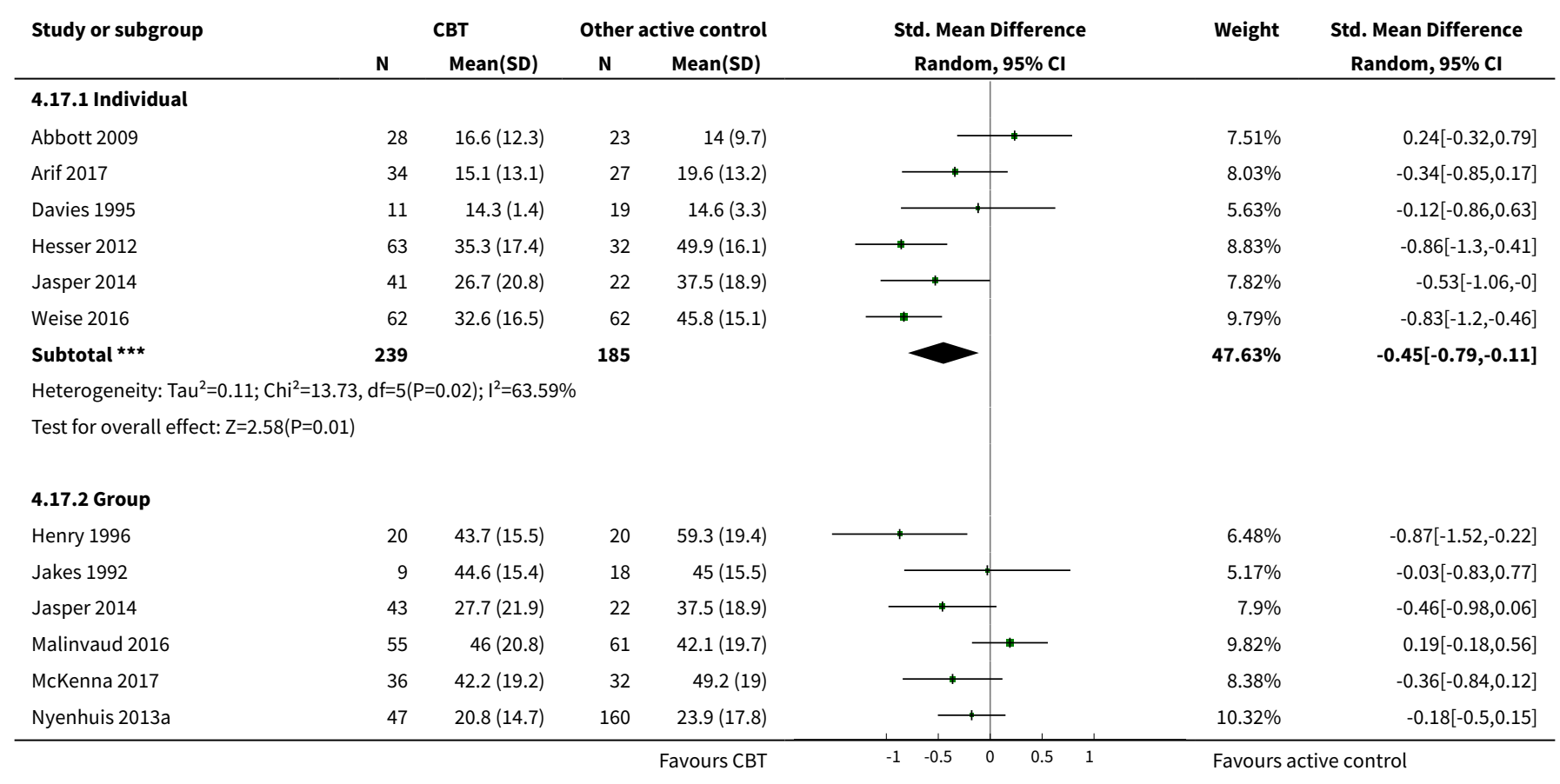




\begin{tabular}{|c|c|c|c|c|c|c|c|}
\hline \multirow{3}{*}{$\begin{array}{l}\text { Study or subgroup } \\
\text { Oron (unpublished) }\end{array}$} & \multicolumn{2}{|c|}{ СВT } & \multicolumn{2}{|c|}{ Other active control } & \multirow{2}{*}{$\begin{array}{c}\text { Std. Mean Difference } \\
\text { Random, } 95 \% \mathrm{Cl}\end{array}$} & \multirow[t]{2}{*}{ Weight } & \multirow{2}{*}{$\begin{array}{l}\text { Std. Mean Difference } \\
\text { Random, } 95 \% \mathrm{Cl}\end{array}$} \\
\hline & $\mathbf{N}$ & $\operatorname{Mean}(S D)$ & $\mathbf{N}$ & $\operatorname{Mean}(S D)$ & & & \\
\hline & 10 & $50.8(20.7)$ & 9 & $38(21.4)$ & \begin{tabular}{l|l} 
\\
\end{tabular} & $4.31 \%$ & $0.58[-0.34,1.51]$ \\
\hline Subtotal $\star \star \star$ & 220 & & 322 & & & $52.37 \%$ & $-0.19[-0.48,0.1]$ \\
\hline \multicolumn{8}{|c|}{ Heterogeneity: Tau $^{2}=0.07 ; \mathrm{Chi}^{2}=12.66, \mathrm{df}=6(\mathrm{P}=0.05) ; \mathrm{I}^{2}=52.6 \%$} \\
\hline \multicolumn{8}{|c|}{ Test for overall effect: $Z=1.29(P=0.2)$} \\
\hline Total *** & 459 & & 507 & & & $100 \%$ & $-0.31[-0.55,-0.08]$ \\
\hline \multicolumn{8}{|c|}{ Heterogeneity: $\mathrm{Tau}^{2}=0.11 ; \mathrm{Chi}^{2}=33.27, \mathrm{df}=12(\mathrm{P}=0) ; \mathrm{I}^{2}=63.93 \%$} \\
\hline \multicolumn{8}{|c|}{ Test for overall effect: $\mathrm{Z}=2.61(\mathrm{P}=0.01)$} \\
\hline \multicolumn{8}{|c|}{ Test for subgroup differences: $\mathrm{Chi}^{2}=1.32, \mathrm{df}=1(\mathrm{P}=0.25), \mathrm{I}^{2}=24.27 \%$} \\
\hline & & & & Favours CBT & $-1 \quad-0.5$ & Favours & e control \\
\hline
\end{tabular}

Analysis 4.18. Comparison 4 CBT versus other active control, Outcome 18 Subgroup analysis: who delivers intervention - impact of tinnitus on quality of life.

\begin{tabular}{|c|c|c|c|c|c|c|c|}
\hline \multirow[t]{2}{*}{ Study or subgroup } & \multicolumn{2}{|c|}{ CBT } & \multicolumn{2}{|c|}{ Other active control } & \multirow{2}{*}{$\begin{array}{c}\text { Std. Mean Difference } \\
\text { Random, } 95 \% \mathrm{Cl}\end{array}$} & \multirow[t]{2}{*}{ Weight } & \multirow{2}{*}{$\begin{array}{c}\text { Std. Mean Difference } \\
\text { Random, } 95 \% \mathrm{Cl}\end{array}$} \\
\hline & $\mathbf{N}$ & $\operatorname{Mean}(S D)$ & $\mathbf{N}$ & Mean(SD) & & & \\
\hline \multicolumn{8}{|l|}{ 4.18.1 Psychologists } \\
\hline Davies 1995 & 11 & $14.3(1.4)$ & 19 & $14.6(3.3)$ & 1 & $5.63 \%$ & $-0.12[-0.86,0.63]$ \\
\hline Henry 1996 & 20 & $43.7(15.5)$ & 20 & $59.3(19.4)$ & & $6.48 \%$ & $-0.87[-1.52,-0.22]$ \\
\hline Jasper 2014 & 43 & $27.7(21.9)$ & 22 & $37.5(18.9)$ & & $7.9 \%$ & $-0.46[-0.98,0.06]$ \\
\hline Malinvaud 2016 & 55 & $46(20.8)$ & 61 & $42.1(19.7)$ & 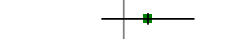 & $9.82 \%$ & $0.19[-0.18,0.56]$ \\
\hline McKenna 2017 & 36 & $42.2(19.2)$ & 32 & $49.2(19)$ & - & $8.38 \%$ & $-0.36[-0.84,0.12]$ \\
\hline Nyenhuis 2013a & 47 & $20.8(14.7)$ & 160 & $23.9(17.8)$ & - & $10.32 \%$ & $-0.18[-0.5,0.15]$ \\
\hline Oron (unpublished) & 10 & $50.8(20.7)$ & 9 & $38(21.4)$ & 1 & $4.31 \%$ & $0.58[-0.34,1.51]$ \\
\hline Weise 2016 & 62 & $32.6(16.5)$ & 62 & $45.8(15.1)$ & & $9.79 \%$ & $-0.83[-1.2,-0.46]$ \\
\hline 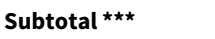 & 284 & & 385 & & & $62.63 \%$ & $-0.29[-0.61,0.02]$ \\
\hline \multicolumn{8}{|c|}{ Heterogeneity: $\mathrm{Tau}^{2}=0.13 ; \mathrm{Chi}^{2}=22.6, \mathrm{df}=7(\mathrm{P}=0) ; \mathrm{I}^{2}=69.02 \%$} \\
\hline \multicolumn{8}{|c|}{ Test for overall effect: $Z=1.85(P=0.06)$} \\
\hline \multicolumn{8}{|l|}{ 4.18.2 Internet-based } \\
\hline Abbott 2009 & 28 & $16.6(12.3)$ & 23 & $14(9.7)$ & $\longrightarrow$ & $7.51 \%$ & $0.24[-0.32,0.79]$ \\
\hline Hesser 2012 & 63 & $35.3(17.4)$ & 32 & $49.9(16.1)$ & & $8.83 \%$ & $-0.86[-1.3,-0.41]$ \\
\hline Jasper 2014 & 41 & $26.7(20.8)$ & 22 & $37.5(18.9)$ & & $7.82 \%$ & $-0.53[-1.06,-0]$ \\
\hline Subtotal $\star \star \star$ & 132 & & 77 & & & $24.17 \%$ & $-0.4[-1.03,0.23]$ \\
\hline \multicolumn{8}{|c|}{ Heterogeneity: $\mathrm{Tau}^{2}=0.24 ; \mathrm{Chi}^{2}=9.2, \mathrm{df}=2(\mathrm{P}=0.01) ; \mathrm{I}^{2}=78.27 \%$} \\
\hline \multicolumn{8}{|c|}{ Test for overall effect: $Z=1.25(P=0.21)$} \\
\hline \multicolumn{8}{|c|}{ 4.18.3 Other 'therapists' } \\
\hline Arif 2017 & 34 & $15.1(13.1)$ & 27 & $19.6(13.2)$ & + & $8.03 \%$ & $-0.34[-0.85,0.17]$ \\
\hline Jakes 1992 & 9 & $44.6(15.4)$ & 18 & $45(15.5)$ & & $5.17 \%$ & $-0.03[-0.83,0.77]$ \\
\hline Subtotal $\star \star \star$ & 43 & & 45 & & & $13.2 \%$ & $-0.25[-0.68,0.18]$ \\
\hline \multicolumn{8}{|c|}{ Heterogeneity: $\operatorname{Tau}^{2}=0 ; \mathrm{Chi}^{2}=0.42, \mathrm{df}=1(\mathrm{P}=0.52) ; \mathrm{I}^{2}=0 \%$} \\
\hline 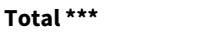 & 459 & & 507 & & & $100 \%$ & $-0.31[-0.55,-0.08]$ \\
\hline \multicolumn{8}{|c|}{ Heterogeneity: $\mathrm{Tau}^{2}=0.11 ; \mathrm{Chi}^{2}=33.27, \mathrm{df}=12(\mathrm{P}=0) ; \mathrm{I}^{2}=63.93 \%$} \\
\hline \multicolumn{8}{|c|}{ Test for overall effect: $Z=2.61(P=0.01)$} \\
\hline Test for subgroup dif & $15, \mathrm{df}=$ & $\mathrm{P}=0.93), \mathrm{I}^{2}=0 \%$ & & & & & \\
\hline
\end{tabular}


Analysis 4.19. Comparison 4 CBT versus other active control, Outcome 19 Subgroup analysis: type of control - impact of tinnitus on quality of life.

\begin{tabular}{|c|c|c|c|c|c|c|c|}
\hline \multirow[t]{2}{*}{ Study or subgroup } & \multicolumn{2}{|c|}{ СВT } & \multicolumn{2}{|c|}{ Other active control } & \multirow{2}{*}{$\begin{array}{c}\text { Std. Mean Difference } \\
\text { Random, } 95 \% \mathrm{Cl}\end{array}$} & \multirow[t]{2}{*}{ Weight } & \multirow{2}{*}{$\begin{array}{c}\text { Std. Mean Difference } \\
\text { Random, } 95 \% \mathrm{Cl}\end{array}$} \\
\hline & $\mathbf{N}$ & $\operatorname{Mean}(S D)$ & $\mathbf{N}$ & Mean(SD) & & & \\
\hline \multicolumn{8}{|l|}{ 4.19.1 Information } \\
\hline Abbott 2009 & 28 & $16.6(12.3)$ & 23 & $14(9.7)$ & 1 & $7.47 \%$ & $0.24[-0.32,0.79]$ \\
\hline Henry 1996 & 20 & $43.7(15.5)$ & 20 & $59.3(19.4)$ & & $6.45 \%$ & $-0.87[-1.52,-0.22]$ \\
\hline Nyenhuis 2013a & 23 & $20.8(14.7)$ & 57 & $27.4(18)$ & $\rightarrow$ & $8.22 \%$ & $-0.38[-0.87,0.11]$ \\
\hline Subtotal $\star \star \star$ & 71 & & 100 & & & $22.14 \%$ & $-0.32[-0.91,0.27]$ \\
\hline \multicolumn{8}{|c|}{ Test for overall effect: $Z=1.07(P=0.29)$} \\
\hline \multicolumn{8}{|c|}{ 4.19.2 Coping effectiveness training } \\
\hline Oron (unpublished) & 10 & $50.8(20.7)$ & 9 & $38(21.4)$ & 1 & $4.31 \%$ & $0.58[-0.34,1.51]$ \\
\hline 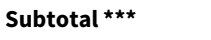 & 10 & & 9 & & & $4.31 \%$ & $0.58[-0.34,1.51]$ \\
\hline \multicolumn{8}{|c|}{ Heterogeneity: Not applicable } \\
\hline \multicolumn{8}{|l|}{ 4.19.3 Relaxation } \\
\hline Arif 2017 & 34 & $15.1(13.1)$ & 27 & $19.6(13.2)$ & 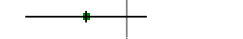 & $7.97 \%$ & $-0.34[-0.85,0.17]$ \\
\hline Davies 1995 & 11 & $14.3(1.4)$ & 19 & $14.6(3.3)$ & & $5.62 \%$ & $-0.12[-0.86,0.63]$ \\
\hline McKenna 2017 & 36 & $42.2(19.2)$ & 32 & $49.2(19)$ & & $8.31 \%$ & $-0.36[-0.84,0.12]$ \\
\hline Subtotal $\star \star \star$ & 81 & & 78 & & & $21.9 \%$ & $-0.31[-0.63,0.01]$ \\
\hline \multicolumn{8}{|c|}{ Heterogeneity: $\operatorname{Tau}^{2}=0 ; \mathrm{Chi}^{2}=0.32, \mathrm{df}=2(\mathrm{P}=0.85) ; \mathrm{I}^{2}=0 \%$} \\
\hline \multicolumn{8}{|c|}{ Test for overall effect: $Z=1.92(P=0.06)$} \\
\hline \multicolumn{8}{|c|}{ 4.19.4 Discussion forum } \\
\hline Hesser 2012 & 63 & $35.3(17.4)$ & 32 & $49.9(16.1)$ & & $8.76 \%$ & $-0.86[-1.3,-0.41]$ \\
\hline Jasper 2014 & 84 & $27.2(21.2)$ & 44 & $37.5(18.9)$ & & $9.66 \%$ & $-0.5[-0.87,-0.13]$ \\
\hline Weise 2016 & 62 & $32.6(16.5)$ & 62 & $45.8(15.1)$ & 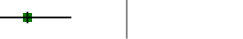 & $9.69 \%$ & $-0.83[-1.2,-0.46]$ \\
\hline \multicolumn{8}{|c|}{ Test for overall effect: $Z=6.09(P<0.0001)$} \\
\hline \multicolumn{8}{|l|}{ 4.19.5 Masking } \\
\hline Jakes 1992 & 9 & $44.6(15.4)$ & 18 & $45(15.5)$ & & $5.16 \%$ & $-0.03[-0.83,0.77]$ \\
\hline Subtotal $\star \star \star$ & 9 & & 18 & & & $5.16 \%$ & $-0.03[-0.83,0.77]$ \\
\hline \multicolumn{8}{|c|}{ Heterogeneity: Not applicable } \\
\hline \multicolumn{8}{|c|}{ Test for overall effect: $Z=0.07(P=0.95)$} \\
\hline \multicolumn{8}{|l|}{ 4.19.6 Virtual reality } \\
\hline Malinvaud 2016 & 55 & $46(20.8)$ & 61 & $42.1(19.7)$ & & $9.72 \%$ & $0.19[-0.18,0.56]$ \\
\hline Subtotal $\star \star \star$ & 55 & & 61 & & & $9.72 \%$ & $0.19[-0.18,0.56]$ \\
\hline \multicolumn{8}{|c|}{ Heterogeneity: Not applicable } \\
\hline \multicolumn{8}{|c|}{ Test for overall effect: $Z=1.02(P=0.31)$} \\
\hline 4.19.7 Self-help & & & & & & & \\
\hline Nyenhuis 2013a & 23 & $20.8(14.7)$ & 103 & $21.9(17.4)$ & & $8.65 \%$ & $-0.06[-0.52,0.39]$ \\
\hline Subtotal $* \star \star$ & 23 & & 103 & & & $8.65 \%$ & $-0.06[-0.52,0.39]$ \\
\hline Heterogeneity: Not a & & & & & & & \\
\hline Test for overall effec & & & & & & & \\
\hline
\end{tabular}




\begin{tabular}{|c|c|c|c|c|c|c|}
\hline \multirow{3}{*}{$\begin{array}{l}\text { Study or subgroup } \\
\text { Total } \star \star \star\end{array}$} & \multicolumn{2}{|c|}{ CBT } & Other active control & \multirow{2}{*}{$\begin{array}{c}\text { Std. Mean Difference } \\
\text { Random, } 95 \% \mathrm{Cl}\end{array}$} & \multirow[t]{2}{*}{ Weight } & \multirow{2}{*}{$\begin{array}{l}\text { Std. Mean Difference } \\
\text { Random, } 95 \% \mathrm{Cl}\end{array}$} \\
\hline & $\mathbf{N}$ & $\operatorname{Mean}(S D)$ & $\operatorname{Mean}(S D)$ & & & \\
\hline & 458 & & 507 & & $100 \%$ & $-0.3[-0.54,-0.06]$ \\
\hline \multicolumn{7}{|c|}{ Heterogeneity: $\mathrm{Tau}^{2}=0.11 ; \mathrm{Chi}^{2}=33.79, \mathrm{df}=12(\mathrm{P}=0) ; \mathrm{I}^{2}=64.49 \%$} \\
\hline \multicolumn{7}{|c|}{ Test for overall effect: $Z=2.5(P=0.01)$} \\
\hline \multicolumn{7}{|c|}{ Test for subgroup differences: $\mathrm{Chi}^{2}=24.16, \mathrm{df}=1(\mathrm{P}=0), \mathrm{I}^{2}=75.16 \%$} \\
\hline
\end{tabular}

Analysis 4.20. Comparison 4 CBT versus other active control, Outcome 20 Sensitivity analysis (fixed-effect model): impact of tinnitus on quality of life at end of treatment.

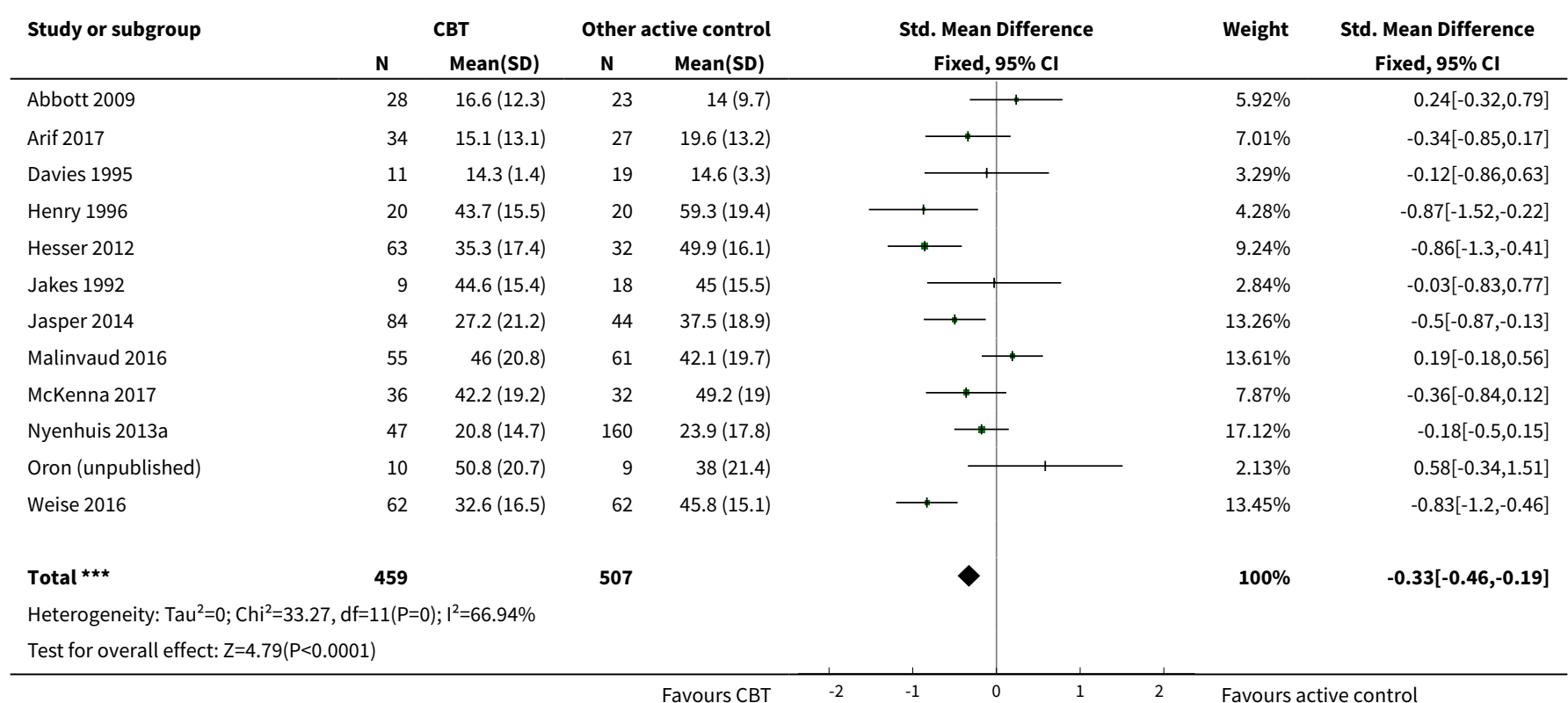

Analysis 4.21. Comparison 4 CBT versus other active control, Outcome 21 Sensitivity analysis: without studies at high risk of bias for incomplete outcome data - impact of tinnitus on quality of life.

\begin{tabular}{|c|c|c|c|c|c|c|c|}
\hline \multirow[t]{2}{*}{ Study or subgroup } & \multicolumn{2}{|c|}{ CBT } & \multicolumn{2}{|c|}{ Other active control } & \multirow{2}{*}{$\begin{array}{c}\text { Std. Mean Difference } \\
\text { Random, } 95 \% \mathrm{Cl}\end{array}$} & \multirow[t]{2}{*}{ Weight } & \multirow{2}{*}{$\begin{array}{c}\text { Std. Mean Difference } \\
\text { Random, } 95 \% \mathrm{Cl}\end{array}$} \\
\hline & $\mathbf{N}$ & Mean(SD) & $\mathbf{N}$ & Mean(SD) & & & \\
\hline Arif 2017 & 34 & $15.1(13.1)$ & 27 & $19.6(13.2)$ & \begin{tabular}{l|l}
1 & \\
\end{tabular} & $10.79 \%$ & $-0.34[-0.85,0.17]$ \\
\hline Henry 1996 & 20 & $43.7(15.5)$ & 20 & $59.3(19.4)$ & & $7.9 \%$ & $-0.87[-1.52,-0.22]$ \\
\hline Hesser 2012 & 63 & $35.3(17.4)$ & 32 & $49.9(16.1)$ & & $12.53 \%$ & $-0.86[-1.3,-0.41]$ \\
\hline Jakes 1992 & 9 & $44.6(15.4)$ & 18 & $45(15.5)$ & & $5.86 \%$ & $-0.03[-0.83,0.77]$ \\
\hline Jasper 2014 & 84 & $27.2(21.2)$ & 44 & $37.5(18.9)$ & & $14.79 \%$ & $-0.5[-0.87,-0.13]$ \\
\hline Nyenhuis 2013a & 150 & $21.6(16.6)$ & 58 & $27.4(18)$ & & $17.06 \%$ & $-0.34[-0.65,-0.04]$ \\
\hline Oron (unpublished) & 10 & $50.8(20.7)$ & 9 & $38(21.4)$ & & $4.66 \%$ & $0.58[-0.34,1.51]$ \\
\hline Weise 2016 & 62 & $32.6(16.5)$ & 62 & $45.8(15.1)$ & 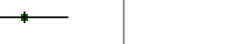 & $14.88 \%$ & $-0.83[-1.2,-0.46]$ \\
\hline Total $\star \star \star$ & 468 & & 302 & & & $100 \%$ & $-0.48[-0.71,-0.26]$ \\
\hline \multicolumn{8}{|c|}{ Heterogeneity: $\mathrm{Tau}^{2}=0.05 ; \mathrm{Chi}^{2}=15.18, \mathrm{df}=8(\mathrm{P}=0.06) ; \mathrm{I}^{2}=47.31 \%$} \\
\hline
\end{tabular}




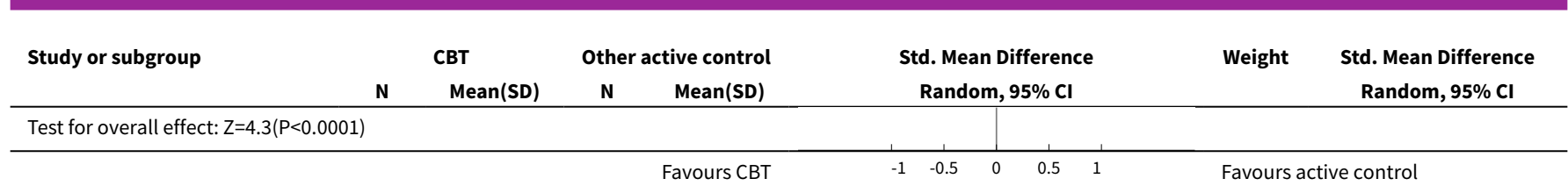

Analysis 4.22. Comparison 4 CBT versus other active control, Outcome 22 Sensitivity analysis: without high risk of bias of missing outcome data, by subgroups (random-effects model): type of therapy - impact of tinnitus on quality of life.

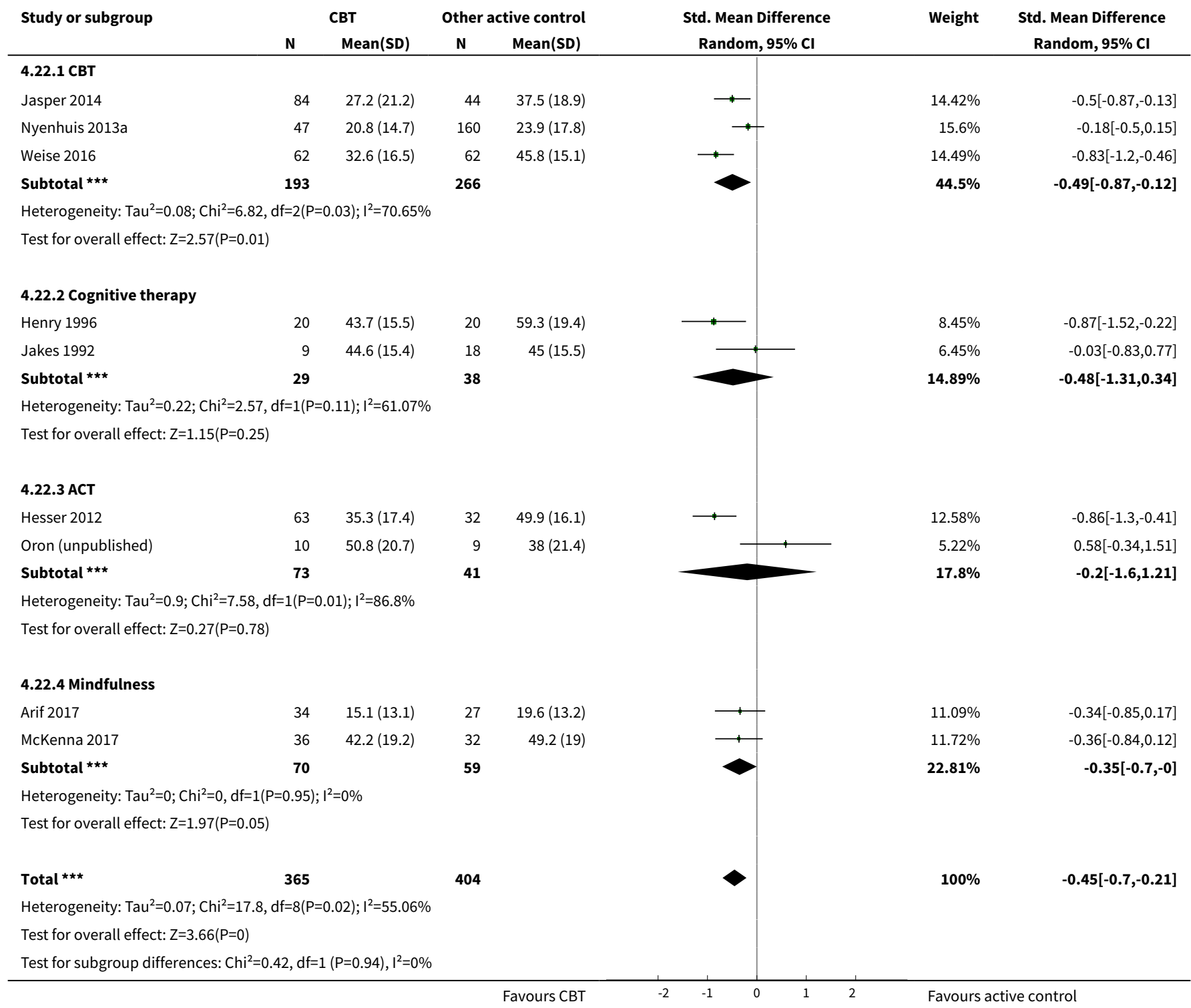


Analysis 4.23. Comparison 4 CBT versus other active control, Outcome 23 Sensitivity analysis: optimistic assumption for Malinvaud - impact of tinnitus on quality of life.

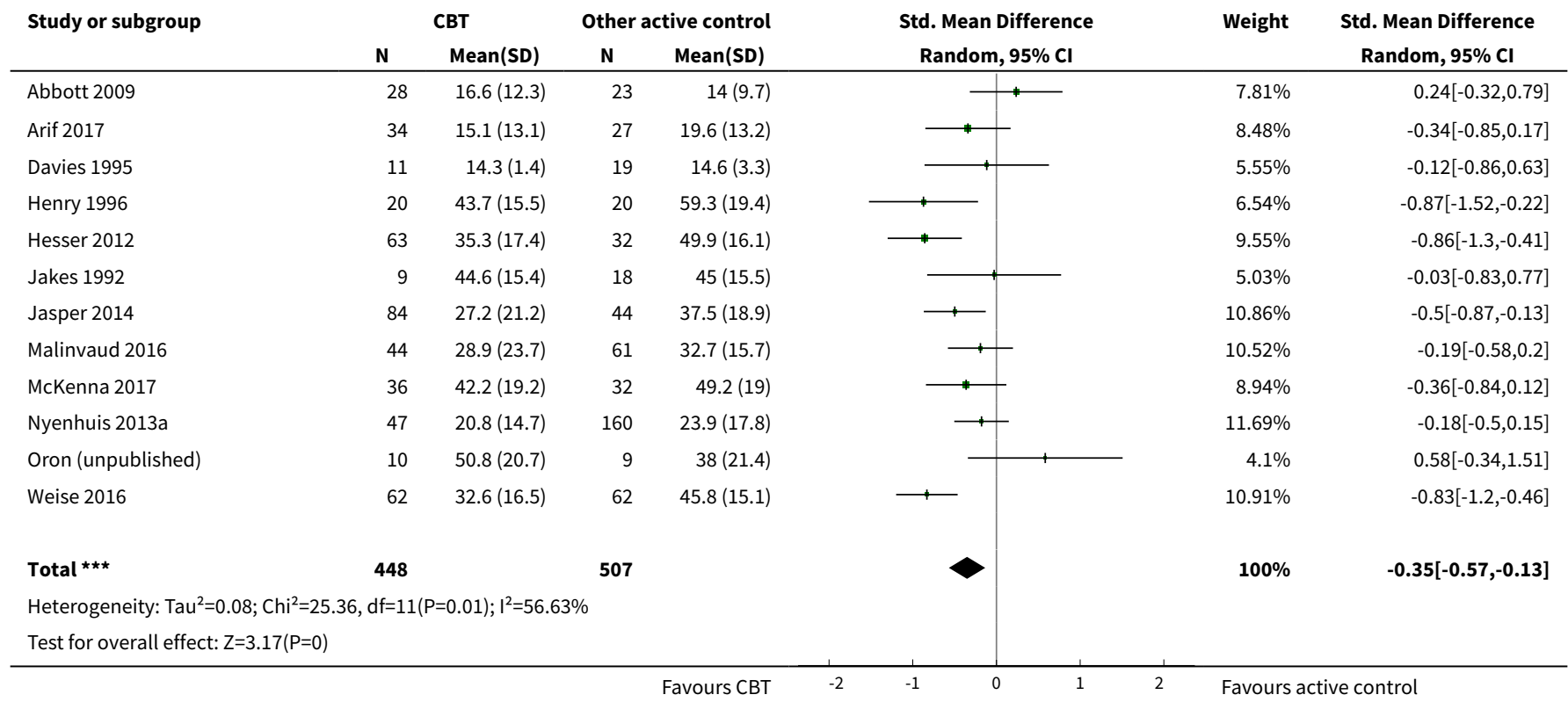

Analysis 4.24. Comparison 4 CBT versus other active control, Outcome 24 Sensitivity analysis: optimistic assumption for Malinvaud - depression.

\begin{tabular}{|c|c|c|c|c|c|c|c|}
\hline \multirow{3}{*}{$\begin{array}{l}\text { Study or subgroup } \\
\text { Abbott } 2009\end{array}$} & \multicolumn{2}{|c|}{ CBT } & \multicolumn{2}{|c|}{ Other active control } & \multirow{2}{*}{$\begin{array}{c}\text { Std. Mean Difference } \\
\text { Random, } 95 \% \mathrm{Cl}\end{array}$} & \multirow[t]{2}{*}{ Weight } & \multirow{2}{*}{$\begin{array}{c}\text { Std. Mean Difference } \\
\text { Random, } 95 \% \mathrm{Cl}\end{array}$} \\
\hline & $\mathbf{N}$ & Mean(SD) & $\mathbf{N}$ & $\operatorname{Mean}(S D)$ & & & \\
\hline & 28 & $4.6(5.3)$ & 23 & $2.4(4.3)$ & \begin{tabular}{l|l} 
&
\end{tabular} & $5.98 \%$ & $0.44[-0.12,1]$ \\
\hline Arif 2017 & 34 & $4.8(3)$ & 27 & $5.2(3.8)$ & & $7.3 \%$ & $-0.1[-0.6,0.41]$ \\
\hline Davies 1995 & 10 & $7.8(7)$ & 18 & $8.3(7.4)$ & & $3.12 \%$ & $-0.06[-0.84,0.71]$ \\
\hline Henry 1996 & 20 & $11.9(6.9)$ & 20 & $11.5(8.6)$ & & $4.86 \%$ & $0.06[-0.56,0.68]$ \\
\hline Hesser 2012 & 63 & $3.4(2.8)$ & 32 & $4.6(3.3)$ & & $10.14 \%$ & $-0.39[-0.81,0.04]$ \\
\hline Malinvaud 2016 & 44 & $4.4(4.1)$ & 50 & $4.6(3.2)$ & & $11.37 \%$ & $-0.04[-0.44,0.37]$ \\
\hline McKenna 2017 & 36 & $6.2(3.1)$ & 32 & $7.5(3.8)$ & - & $8.08 \%$ & $-0.37[-0.85,0.11]$ \\
\hline Nyenhuis 2013a & 47 & $4.7(4.8)$ & 160 & $5.7(5.1)$ & - & $17.59 \%$ & $-0.2[-0.53,0.12]$ \\
\hline Philippot 2012a & 13 & $8.8(9.4)$ & 12 & $12.8(5.4)$ & & $2.92 \%$ & $-0.5[-1.3,0.3]$ \\
\hline Weise 2016 & 62 & $5.3(3.7)$ & 62 & $6.7(4)$ & & $14.82 \%$ & $-0.36[-0.71,-0]$ \\
\hline \multicolumn{8}{|c|}{ Heterogeneity: $\mathrm{Tau}^{2}=0 ; \mathrm{Chi}^{2}=9.86, \mathrm{df}=10(P=0.45) ; \mathrm{I}^{2}=0 \%$} \\
\hline \multicolumn{3}{|c|}{ Test for overall effect: $Z=2.97(P=0)$} & & & & & \\
\hline
\end{tabular}


Analysis 4.25. Comparison 4 CBT versus other active control, Outcome 25 Sensitivity analysis: optimistic assumption for Malinvaud - anxiety.

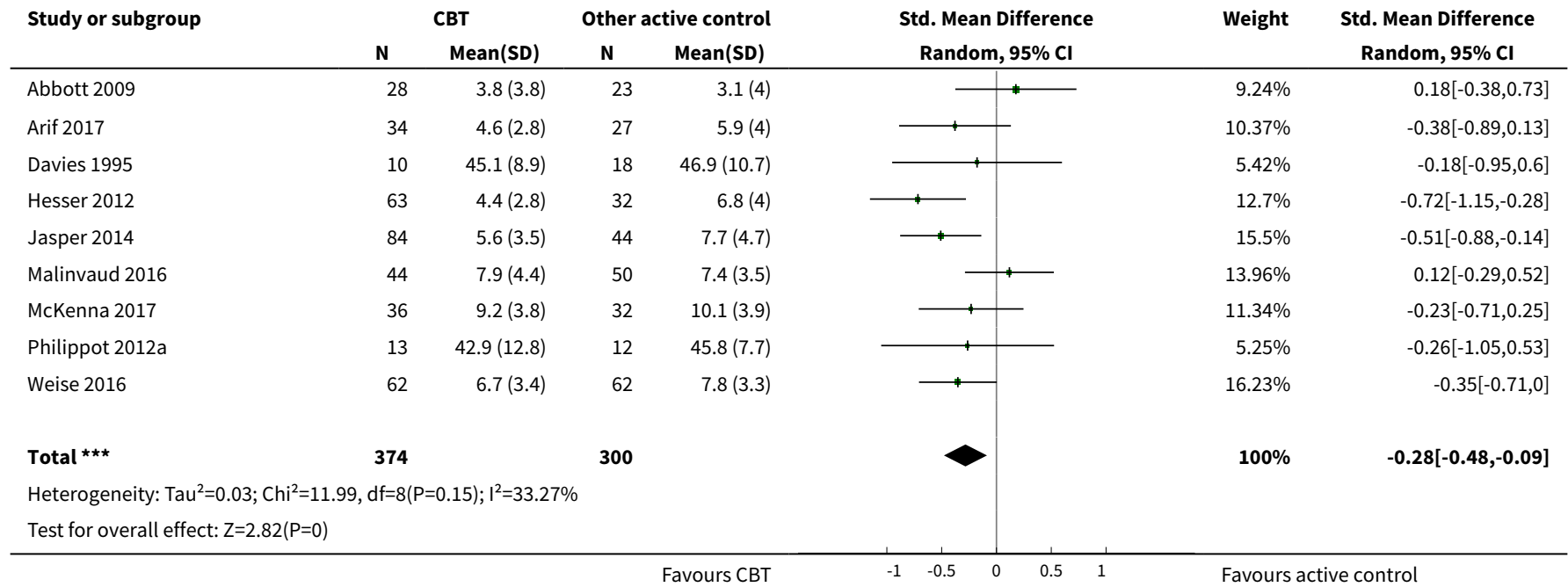

\section{APPENDICES}

\section{Appendix 1. Pathophysiology of tinnitus}

In the central auditory system, tinnitus-related alterations have been described along the whole central auditory pathway including the dorsal cochlear nucleus (Middleton 2011; Pilati 2012), the inferior colliculus (Dong 2010; Mulders 2010), and the auditory and nonauditory cortex (for review see Elgoyhen 2015). There is a strong rationale that these structural and functional alterations are a direct consequence of maladaptive neuroplastic responses to hearing loss (Møller 2000; Mühlnickel 1998), or to altered somatosensory input from the face or the neck (Shore 2016). Presumably sensory deafferentation triggers a release from inhibition in the central auditory system resulting in spontaneous hyperactivity and increased spontaneous synchronous activity within the central neuronal networks involved in sound processing (Dietrich 2001; Eggermont 2004; Rauschecker 1999; Schaette 2011; Seki 2003; Tass 2012; Weisz 2005). Another physiological change thought to be related to tinnitus generation is a process of functional reorganisation. This amounts to a change in the response properties of neurons within the primary auditory cortex to external sounds. This effect is well demonstrated physiologically in animal models of hearing loss (Engineer 2011; Noreña 2005). Evidence in humans, however, is limited to behavioural evidence of cortical reorganisation after hearing loss, demonstrating improved frequency discrimination ability at the audiometric edge (Kluk 2006; McDermott 1998; Moore 2009; Thai-Van 2002; Thai-Van 2003), although Buss 1998 did not find this effect. Imaging studies in tinnitus patients without hearing loss as shown in a normal audiogram did not demonstrate functional reorganisation of the brain's auditory system macroscopically altered tonotopic organisation (Langers 2012), indicating that altered tonotopic organisation is rather a consequence of hearing loss and not causally related to tinnitus. This indicates that such reorganisation is a consequence of hearing loss, but is not sufficient to cause tinnitus.

\section{Appendix 2. Psychological models of the effects of tinnitus}

Several influential models have been proposed to explain the development and maintenance of distress and interference associated with chronic subjective tinnitus. Each of the models are briefly described here as they underlie the development of and rationale for applying cognitive behavioural therapy for the treatment of the impact of tinnitus on quality of life.

The concept of habituation - a process whereby reaction(s) decrease in response to repeated presentation of a stimulus (Bouton 2007) was first applied in 1984 by Hallam and colleagues to explain reduction in the impact of tinnitus on quality of life over time. They proposed that for most people repeated perception of the tinnitus sound led them to learn that the stimulus was not worthy of attentional resources (Hallam 1984). However, lower tinnitus-related quality of life occurs when there are failures in these attentional processes that might especially happen at times of stress and high arousal, which put strain on cognitive resources (Mazurek 2015). Operant conditioning (Skinner 1938), which attributes importance to the consequences of actions, was later included in the model to account for learning mechanisms and avoidant behaviours (Kröner-Herwig 2003). The difficulty for the person arises though when significant or continuous resources (cognitive or otherwise) are needed to avoid the tinnitus to experience relief. To treat the impact of tinnitus on quality of life (or facilitate habituation to tinnitus), it was recommended that stress levels and central nervous system arousal levels should be reduced 
in order to change the meaning of the tinnitus signal for the patient (McKenna 2004). To date there is mixed evidence in support of the habituation model (Baguley 2013).

Jastreboff expanded this model by postulating that the association between tinnitus and an aversive emotional state emerges through classical conditioning mechanisms (Jastreboff 1988; Jastreboff 1990). Classical (or Pavlovian) conditioning refers to a process whereby a person learns a relationship between the two stimuli, a neutral one (conditioned stimulus) and a biologically relevant one (unconditioned stimulus) (Pavlov 1927). Subsequent presentation of either will activate the representation of the biologically relevant one and elicit a conditioned response. While Jastreboff described how an association developed between the tinnitus perception and an aversive emotional state, it was not clearly specified what the unconditioned stimulus, conditioned stimulus and conditioned responses respectively were (Baguley 2013). Regardless, to counter the effect, treatment should aim to break the negative association with the tinnitus percept by using directive cognitive therapy and sound therapy (Jastreboff 1993; Jastreboff 2004).

More recently a cognitive model (McKenna 2014), and cognitive-behavioural (i.e. fear avoidance) model (Cima 2011b; Kleinstauber 2013; Lethem 1983; Vlaeyen 2000; Vlaeyen 2012), have been applied to tinnitus. The cognitive model stresses the importance of primary and secondary cognitive appraisals and the effect on attentional processes (McKenna 2014). The negative evaluation of the tinnitus can be viewed as being comprised of primary and secondary appraisals. That is, a person might initially appraise the tinnitus as being threatening to their health, and then make a secondary appraisal of their (in)ability to control it. The fear avoidance model of tinnitus shares features with both the neurophysiological and the cognitive model including attributing a fundamental role to the negative evaluation of tinnitus. The fear avoidance model offers predictions about behavioural factors (e.g. safety behaviours) in the maintenance of lower tinnitus related quality of life. It is proposed that regardless of the cause of the tinnitus, once it is detected, attention, cognitive appraisals and emotional reactions elicit behavioural responses, which are relieving in the short term but paradoxically lead to severe impairment in the long term.

In the fear avoidance model, the role of fear reactions and safety behaviours is purported to be the key mechanism in the maintenance of chronic tinnitus suffering (Cima 2011b; Kleinstauber 2013). Its central tenet is that the main reactions to tinnitus depend on the initial response. In case of misinterpretations, increased threat value will be associated with tinnitus. That is to say, negative autonomic psychophysiological reactivity may lead to catastrophic (mis)interpretations (i.e. a bias towards misinterpreting the tinnitus as something extremely harmful). Fear responses, such as avoidance and escape tendencies, will in turn lead to task-interference, depression, inactivity and ultimately to severe impairment in daily life (Cima 2011a; Cima 2011b). These fear behaviours are reinforced since they offer relief by reducing fear and acute reactivity in the short term, but unfortunately prolong fear-avoidance responsiveness and therefore impairment in the long term.

Although these psychological models slightly differ in their main premise and in some of the terminology used, they all identify mechanisms, either of a cognitive and/or behavioural nature, which have been targeted in therapy to tinnitus related quality of life.

\section{Appendix 3. Tinnitus measurement tools}

There are numerous tools used for tinnitus evaluation including the Tinnitus Questionnaire (TQ) (Hallam 1988), the Tinnitus Reaction Questionnaire (TRQ) (Wilson 1991), the Tinnitus Functional Index (TFI) (Meikle 2012) and the Tinnitus Handicap Inventory (THI) (Newman 1996). For a discussion of the development and validity of questionnaires for measuring the impact of tinnitus on quality of life see Fackrell 2014.

For illustrative purposes, the THI is presented below.

The THI is a self-administered tool to measure the impact of the tinnitus in daily life (Newman 1996). It consists of 25 items that may be answered yes (four points), sometimes (two points) or no (zero points), summing up a total of 100 points, with higher scores corresponding to a higher handicap. The items are divided into three subscales:

- The functional subscale (F) (11 items) encompasses role limitations in the areas of mental functioning, social/occupational functioning and physical functioning.

- The emotional subscale (E) (nine items) includes items addressing affective responses to tinnitus (anger, frustration, irritability, depression).

- The catastrophic subscale (C) (five items) reflects patients' desperation, inability to escape from tinnitus, perception of having a terrible disease, lack of control and inability to cope.

1. Because of your tinnitus is it difficult for you to concentrate? (F)

2. Does the loudness of your tinnitus make it difficult for you to hear people? (F)

3. Does your tinnitus make you angry? (E)

4. Does your tinnitus make you confused? (F)

5. Because of your tinnitus are you desperate? (C)

6. Do you complain a great deal about your tinnitus? (E)

7. Because of your tinnitus do you have trouble falling asleep at night? (F)

8. Do you feel as though you cannot escape from your tinnitus? (C)

9. Does your tinnitus interfere with your ability to enjoy social activities (such as going out to dinner, to the cinema)? (F) 
10.Because of your tinnitus do you feel frustrated? (E)

11.Because of your tinnitus do you feel that you have a terrible disease? (C)

12.Does your tinnitus make it difficult to enjoy life? (F)

13.Does your tinnitus interfere with your job or household responsibilities? (F)

14. Because of your tinnitus do you find that you are often irritable? (F)

15. Because of your tinnitus is it difficult for you to read? (F)

16. Does your tinnitus make you upset? (E)

17. Do you feel that your tinnitus has placed stress on your relationships with members of your family and friends? (E)

18.Do you find it difficult to focus your attention away from your tinnitus and on to other things? (F)

19.Do you feel that you have no control over your tinnitus? (C)

20.Because of your tinnitus do you often feel tired? (F)

21.Because of your tinnitus do you feel depressed? (E)

22.Does your tinnitus make you feel anxious? (E)

23.Do you feel you can no longer cope with your tinnitus? (C)

24.Does your tinnitus get worse when you are under stress? (F)

25.Does your tinnitus make you feel insecure? (E)

According to the score, tinnitus can be classified into five categories:

Category 1: 0 to 16. Slight (only heard in quiet environments).

Category 2: 18 to 36 . Mild (easily masked by environmental sounds and easily forgotten with activities).

Category 3: 38 to 56. Moderate (noticed in the presence of background noise, though daily activities can still be performed).

Category 4: 58 to 76. Severe (almost always heard, leads to disturbed sleep patterns and can interfere with daily activities).

Category 5: 78 to 100. Catastrophic (always heard, disturbed sleep patterns, difficulty with any activities).

\section{Appendix 4. Outcome measures and citations}

- Tinnitus Questionnaire (Hallam 1988; Hallam 2008).

- German version of Tinnitus Questionnaire (Goebel 1994).

- Tinnitus Functional Index (Meikle 2012).

- Tinnitus Handicap Inventory (Newman 1996).

- Tinnitus Handicap Questionnaire (Kuk 1990).

- Tinnitus Reaction Questionnaire (Wilson 1991).

- Tinnitus Severity Scale (Sweetow 1990).

- Tinnitus Disability Index (Cima 2011a).

\section{Appendix 5. Search strategies}

\begin{tabular}{|c|c|c|}
\hline CENTRAL (CRS Web) & MEDLINE (Ovid) & Embase (Ovid) \\
\hline \multirow[t]{2}{*}{$\begin{array}{l}\text { \#1 MESH DESCRIPTOR Tinnitus EXPLODE ALL AND CEN- } \\
\text { TRAL:TARGET }\end{array}$} & 1. exp Tinnitus/ & 1 exp Tinnitus/ \\
\hline & 2. "tinnit" .ab,ti. & 2 "tinnit*".ab,ti. \\
\hline 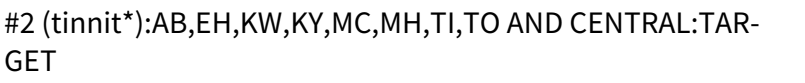 & 3. exp Behavior Therapy/ & 3 exp behavior therapy/ \\
\hline \multirow{2}{*}{$\begin{array}{l}\text { \#3 MESH DESCRIPTOR Behavior Therapy EXPLODE ALL AND } \\
\text { CENTRAL:TARGET }\end{array}$} & 4. exp Adaptation, Psychological/ & 4 exp adaptation/ \\
\hline & 5. exp Meditation/ & 5 exp meditation/ \\
\hline \multirow{3}{*}{$\begin{array}{l}\text { \#4 MESH DESCRIPTOR Adaptation, Physiological EXPLODE } \\
\text { ALL AND CENTRAL:TARGET } \\
\text { \#5 MESH DESCRIPTOR Meditation EXPLODE ALL AND CEN- } \\
\text { TRAL:TARGET }\end{array}$} & $\begin{array}{l}\text { 6. (CBT or ACT or mindfulness or MBTR or } \\
\text { MBSR or MBTSR or psychoeducation or }\end{array}$ & $\begin{array}{l}6 \text { (CBT or ACT or mind- } \\
\text { fulness or MBTR or }\end{array}$ \\
\hline & $\mathrm{i} A C T$ or iCBT or GCBT).ab,ti. & MBSR or MBTSR or psy- \\
\hline & 7. ((cogniti ${ }^{\star}$ or relaxation or acceptance & $\begin{array}{l}\text { choeducation or iACT or } \\
\text { iCBT or GCBT).ab,ti. }\end{array}$ \\
\hline $\begin{array}{l}\text { \#6 (CBT or ACT or mindfulness or MBTR or MBSR or MBTSR or } \\
\text { psychoeducation or iACT or iCBT or GCBT):AB,EH,KW,KY,M- } \\
\text { C,MH,TI,TO AND CENTRAL:TARGET }\end{array}$ & $\begin{array}{l}\text { or commitment or adaptation) adj6 (ther- } \\
\text { ap }^{\star} \text { or behavior* or behaviour* or strateg* } \\
\text { or intervention* or approach* or psy- }\end{array}$ & $\begin{array}{l}7\left(\left(\text { cogniti }{ }^{\star} \text { or relax- }\right.\right. \\
\text { ation or acceptance or }\end{array}$ \\
\hline
\end{tabular}

Cognitive behavioural therapy for tinnitus (Review) 
(Continued)

\#7 ((cogniti* or relaxation or acceptance or commitment or adaptation) near (therap* or behavior* or behaviour* or strateg $^{\star}$ or intervention* or approach * or psychotherap* or training or treatment or technique* or program * or counselling or counselling)):AB,EH,KW, KY,MC,MH,TI,TO AND CENTRAL:TARGET

\#8 ((behaviour* or behavior* or meditation) near (strateg* or intervention * or therap* or approach* or psychotherap* or technique* or counselling or counselling)):AB,EH, KW, KY,MC,MH,TI,TO AND CENTRAL:TARGET

\#9 \#1 OR \#2

\#10 \#8 OR \#7 OR \#6 OR \#5 OR \#4 OR \#3

\#11 \#9 AND \#10 chotherap* or training or treatment or technique* or program * or counselling or counselling)).ab,ti.

8. ((behaviour ${ }^{\star}$ or behavior* or meditation) adj6 (strateg* or intervention* or therap * or approach * or psychotherap* or technique ${ }^{\star}$ or counselling or counselling)).ab,ti.

\section{1 or 2}

10.3 or 4 or 5 or 6 or 7 or 8

11.9 and 10

commitment or adaptation) adj6 (therap* or behavior $^{\star}$ or behaviour ${ }^{\star}$ or strateg $^{\star}$ or intervention* or approach* or psychotherap* or training or treatment or technique* or program * or counselling or counselling)).ab,ti.

8 ((behaviour ${ }^{\star}$ or behavior* or meditation) adj6 (strateg* or intervention* or therap* or approach* or psychotherap* or technique* or counselling or counselling)).ab,ti.

91 or 2

103 or 4 or 5 or 6 or 7 or 8

119 and 10

\section{ClinicalTrials.gov}

\section{CT.gov}

Condition: tinnitus

S10 S7 OR S8

\section{S9 S1 OR S2 OR S3 OR S4 OR S5 OR S6}

\section{S8 TX tinnit*}

\section{S7 (MH "tinnitus")}

S6 TX (behaviour* or behavior ${ }^{\star}$ or meditation) n6 (strateg* or intervention* or therap* or approach* or psychotherap* or technique* or counseling or counselling)

S5 TX (cogniti* or relaxation or acceptance or commitment or adaptation) n6 (therap ${ }^{\star}$ or behavior ${ }^{\star}$ or behaviour ${ }^{\star}$ or strateg $^{\star}$ or intervention * or approach ${ }^{\star}$ or psychotherap* or training or treatment or technique* or program * or counseling or counselling)

S4 TX CBT or ACT or mindfulness or MBTR or MBSR or MBTSR or psychoeducation or iACT or iCBT or GCBT

S3 (MH "Meditation+")

S2 (MH "Behavior Therapy+")
S1 (MH "Adaptation, Psychological+")
Study Type: Interventional
Indexes=SCI-EXPANDED, SSCI, CPCI-S, CPCI-SSH Timespan=All years

\#5 \#4 OR \#3 OR \#2

Indexes=SCI-EXPANDED, SSCl, CPCI-S, CPCI-SSH Timespan=All years

\#6 \#5 AND \#1 
(Continued)

\section{CONTRIBUTIONS OF AUTHORS}

The author contributions were as follows:

- TF, RC and JWSV conceived and all authors contributed to the design of the study.

- The Cochrane ENT Information Specialist developed and ran the search strategy.

- TF obtained copies of the studies with the assistance of Maastricht University Library.

- TF, RC and DH were responsible for the selection of studies.

- TF, RC, BM and DH were responsible for data extraction.

- TF, RC, BM and DH were responsible for assessing risk of bias.

- TF entered data into RevMan.

- TF, RC and DH, in consultation with Cochrane Methods Support Unit, conducted the analysis.

- All authors contributed to the interpretation of the analysis.

- TF drafted and revised the review and all authors commented critically on intellectual content.

- All authors will contribute to the drafting and updating of the review.

\section{DECLARATIONS OF INTEREST}

Where review authors were also study authors, they had no involvement in study selection, data extraction or risk of bias assessment.

Thomas Fuller: none known.

Rilana Cima: was an investigator and author of the Cima 2012 study, which was a RCT comparing stepped CBT-based care with treatment as usual on the impact of tinnitus on quality of life.

Berthold Langguth: has received funding for research from the Deutsche Forschungsgemeinschaft, the German Ministry for Research, the American Tinnitus Association, the Tinnitus Research Initiative, the European Union, Otonomy and Sivantos. He has received consultancy and speaker honoraria from Autifony, ANM, Astra Zeneca, Kyorin, Merz, McKinsey, Microtransponder, Neuromod, Novartis, Pfizer, Lundbeck and Servier.

Birgit Mazurek: has received funding for research from the Deutsche Forschungsgemeinschaft, the German Ministry for Research, the American Tinnitus Association and the German Tinnitus Association Charité.

Derek J Hoare: is vice chair of the British Society of Audiology.

Johan WS Vlaeyen: was an investigator and author of the Cima 2012 study, which was a RCT comparing stepped CBT-based care with treatment as usual on the impact of tinnitus on quality of life.

\section{SOURCES OF SUPPORT}

\section{Internal sources}

- No sources of support supplied

\section{External sources}

- National Institute for Health Research, UK.

Infrastructure funding for Cochrane ENT

- SWOL Limburgs Fonds voor Revalidatie and Netherlands Organisation for Health Research and Development (ZonMW), Netherlands.

Research programme: Health Care Efficiency, Subprogramme: Effects \& Costs, Grant number: 945-07-715 provided funding for the employment of Thomas Fuller 


\section{DIFFERENCES BETWEEN PROTOCOL AND REVIEW}

"Tinnitus reactivity", defined as being tinnitus-specific health-related quality of life, as measured by multi-item questionnaires (e.g. TFI), was specified as the primary outcome in our protocol (Fuller 2017b). This terminology was replaced in the review with "impact of tinnitus on quality of life" in order to be consistent with other reviews related to tinnitus in the Cochrane ENT group (Sereda 2018; Wegner 2018).

We had not specified in our protocol what outcome measure we would use if studies used more than one multi-item questionnaire to measure the secondary outcomes for depression, anxiety, quality of life and negatively biased interpretations of tinnitus. Whenever possible we chose the multi-item questionnaire that would allow pooling of data and analysis using mean differences. Failing that, we chose the measure that had the better psychometric properties.

In the protocol we had not clearly described how we would treat cluster-RCTs that did not include information about the intracluster correlation coefficient (ICC). We followed the recommendations in Chapter 16.3.4 'Approximate analyses of cluster-randomised trials for a meta-analysis: effective sample sizes' in the Cochrane Handbook for Systematic Reviews of Interventions (Higgins 2011). After a search for examples of cluster-RCTs with tinnitus patients yielded no results, we contacted the Cochrane ENT group Managing Editor for further guidance. Courtesy of the Cochrane Methods Support Unit, we obtained an estimate of an ICC that was reasonable for us to use with this population. We also conducted a sensitivity analysis including and excluding the data from Abbott 2009.

We have provided some clarification in the report of the review regarding 'mindfulness'. We have added the following text: "We considered interventions as 'mindfulness' if they involved: exercises that involved self-regulation of attention on experience and emphasised openness, curiosity and acceptance (Bishop 2004)".

In the protocol we stated that if the $\mathrm{I}^{2}$ was $>30 \%$, the $\mathrm{Chi}^{2}$ value was greater than the degrees of freedom and/or the confidence intervals of the included studies did not show overlap, we would not pool studies and instead describe the findings in a narrative form. Although the $\mathrm{I}^{2}$ values were above $30 \%$ in numerous analyses, there was clear overlap of almost all the confidence intervals, and the trend of the effect across studies was consistently towards the interventions favouring CBT regardless of the comparator. Furthermore, we expected that the underlying reason for this high level of statistical heterogeneity was the anticipated clinical or methodological factors for which we had planned subgroup analyses. Given this and the potential value of a meta-analysis compared with only providing a narrative summary of the literature, we pooled the data.

We conducted an additional sensitivity analysis to examine the effect of using a 'conservative' compared to an 'optimistic' approach in one study (Malinvaud 2016), which did not report outcome data at the end of treatment but did so at three months follow-up.

Compared with what we initially described in the protocol, there were changes in the contributions of authors to two tasks. DH also contributed to data extraction, and BM and RC also conducted 'Risk of bias' assessments. AW withdrew from the review team due to increased work commitments elsewhere.

\section{NOTES}

The publication of this review will lead to the previous one becoming obsolete. A link to the superseded review will be available (MartinezDevesa 2010).

\section{N DEX TERMS}

\section{Medical Subject Headings (MeSH)}

${ }^{\star}$ Cognitive Behavioral Therapy [methods]; Anxiety [therapy]; Depression [therapy]; Quality of Life; Randomized Controlled Trials as Topic; Tinnitus [ ${ }^{*}$ psychology] [*therapy]; Treatment Outcome; Waiting Lists

\section{MeSH check words}

Humans 